\title{
Characterization of microglial Rab7 knockout on amyloid pathology in the 5xFAD mouse model of Alzheimer's disease
}

\section{Dissertation}

\author{
in partial fulfillment for the award of the degree \\ "Doctor rerum naturalium" \\ of the Georg-August University Göttingen
}

within the doctoral program "Molecular Physiology of the Brain" of the Georg-August University School of Science (GAUSS)

Submitted by

Beate Maren Erika Koch

From Schwäbisch Hall 


\section{Thesis Committee:}

Prof. Dr. Anja Schneider (Reviewer)

German Center for Neurodegenerative Diseases (DZNE), Bonn

Department of Neurodegenerative Diseases and Geriatric Psychiatry, University Hospital Bonn, Bonn

\section{Prof. Dr. Silvio Rizzoli (Reviewer)}

Dept. of Neuro-and Sensory Physiology, University Medical Center Göttingen, Göttingen

\section{Prof. Dr. André Fischer}

German Center for Neurodegenerative Diseases (DZNE), Göttingen

Department of Psychiatry and Psychotherapy, University Medical Center Göttingen, Göttingen

\section{Members of the Examination Board:}

Prof. Dr. Mikael Simons

Institute of Neuronal Cell Biology (TUM-NZB), München

Technical University of Munich, München

German Center for Neurodegenerative Diseases (DZNE), München

\section{Prof. Dr. Dr. Hannelore Ehrenreich}

Max-Planck-Institute for Experimental Medicine, Clinical Neuroscience, Göttingen

\section{Prof. Dr. Alexander Flügel}

Institute for Neuroimmunology and Multiple Sclerosis Research, University Medical Center Göttingen, Göttingen

\section{Date of oral examination:}

$20^{\text {th }}$ November 2018 


\section{Affidavit}

I hereby declare that I have written my doctoral thesis entitled "Characterization of microglial Rab7 knockout on amyloid pathology in the 5xFAD mouse model of Alzheimer's disease" independently with no other sources and aids than quoted.

Furthermore, I confirm that this thesis has not been submitted as part of another examination process neither in identical nor in similar form.

Bonn,

Beate Koch 


\section{List of Figures}

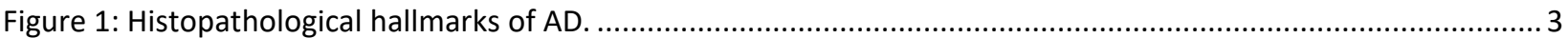

Figure 2: Thal stages of amyloid $(A \beta)$ and Braak stages of NFT pathology. ...................................................... 4

Figure 3: Schematic presentation of non-amyloidogenic, amyloidogenic and $\eta$-secretase pathway........................ 6

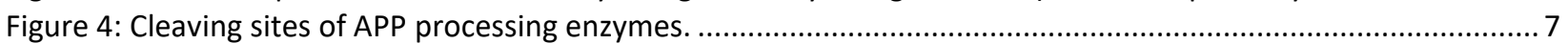

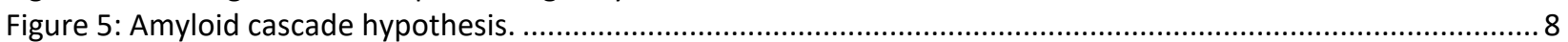

Figure 6: APP sequence with mutation sites found in FAD. ......................................................................... 12

Figure 7: Microglia development and maintenance in the rodent brain. ......................................................... 15

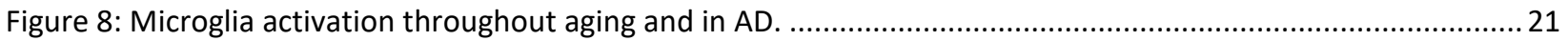

Figure 9: Expression of AD risk genes and endo-lysosomal degradation in microglia. ..........................................25

Figure 10: Schematic role of Rab7 on endo-lysosomal degradation and autophagy. ...........................................27

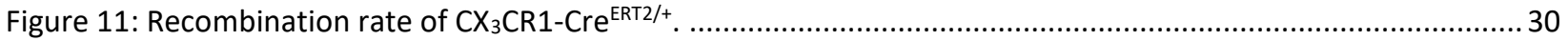

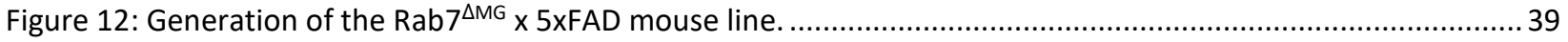

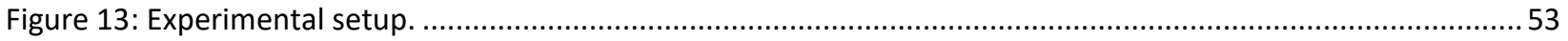

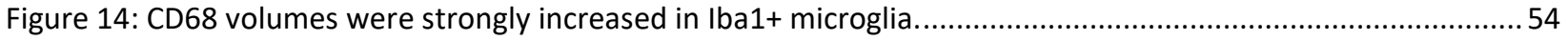

Figure 15: LAMP1+ lysosomes were enlarged in Rab7 ${ }^{\Delta M G}$ x 5xFAD microglia...................................................55

Figure 16: $A \beta$ clearance was disrupted in Rab7 ${ }^{\triangle M G}$ x 5xFAD microglia. ................................................................56

Figure 17: $A \beta$-plaque numbers were reduced in aged Rab7 ${ }^{\Delta \mathrm{MG}}$ x 5xFAD females.................................................58

Figure 18: $A \beta$-plaques were smaller and showed altered morpholgy in Rab7 ${ }^{\triangle M G}$ x 5xFAD females.........................60

Figure 19: Workflow of sequential $A \beta$ isolation from brain homogenates.........................................................61

Figure 20: MSD analysis of 3 month old females did not show differences in the aggregation states of $A \beta_{38}$, $A \beta_{40}$ and $A \beta_{42}$.

Figure 21: The amounts of soluble and insoluble $A \beta_{38}, A \beta_{40}$ and $A \beta_{42}$ in 9 month old Rab7 ${ }^{\Delta M G} \times 5 \times F A D$ females

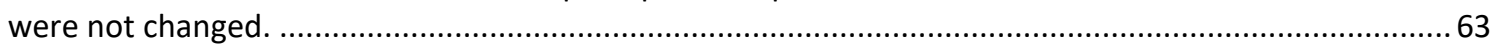

Figure 22: No differences in the aggregation states of $A \beta_{38}, A \beta_{40}$ and $A \beta_{42}$ were found in 3 month old Rab7 $7^{\Delta M G} x$

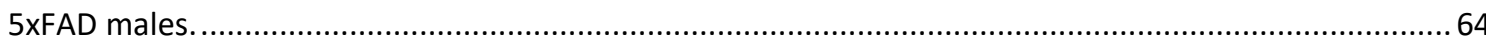

Figure 23: Aggregation states of $A \beta_{38}, A \beta_{40}$ and $A \beta_{42}$ were not changed in 9 month old Rab7 ${ }^{\Delta M G} \times 5 \times F A D$ males......65

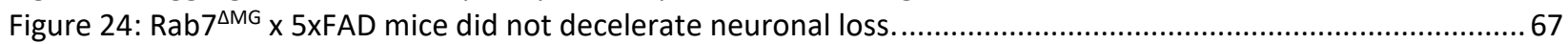

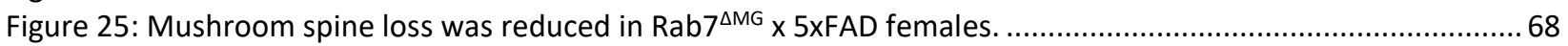

Figure 26: Synaptic loss was not decelerated by Rab7 ${ }^{\triangle M G}$ in $5 x F A D$ mice. ........................................................69.

Figure 27: Cell numbers and recruitment of Iba1+ microglia to $A \beta$-plaques were not affected in 3 month $\mathrm{Rab}^{\triangle \mathrm{MG}}$ x 5xFAD mice.

Figure 28: Cell numbers and recruitment of Iba1+ microglia to $A \beta$-plaques were not impaired in 9 month old Rab7 ${ }^{\triangle M G}$ x 5xFAD mice.

Figure 29: Morphology of Rab7 ${ }^{\triangle M G}$ x 5xFAD microglia was not altered............................................................... 73

Figure 30: Protein profiling identified upregulation of CCL2 in aged Rab7 ${ }^{\triangle M G} \times 5 x F A D$ mice............................... 75

Figure 31: Astrogliosis was not changed in Rab7 ${ }^{\triangle \mathrm{MG}}$ x 5xFAD mice. ........................................................... 77

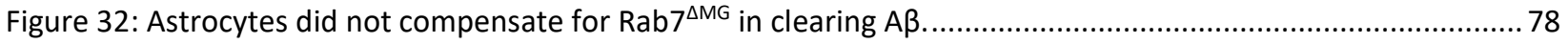

Figure 33: Clearance of $A \beta$ by the blood circulation is not altered in Rab7 ${ }^{\triangle M G} \times 5 x F A D$ mice.............................. 79 


\section{List of Tables}

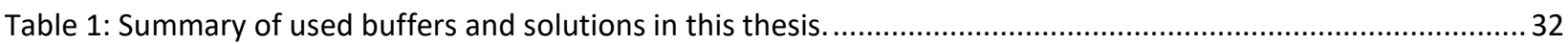

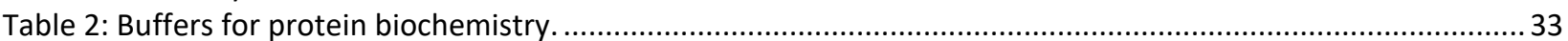

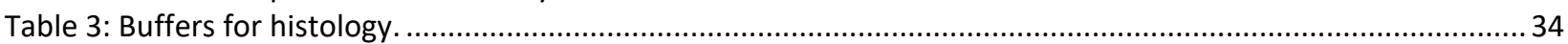

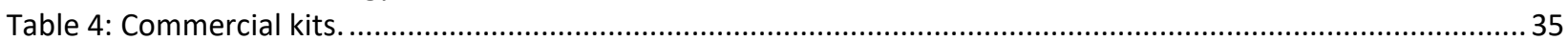

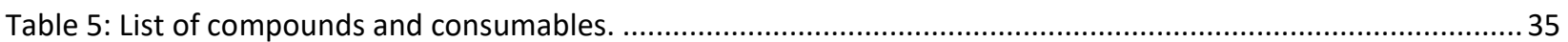

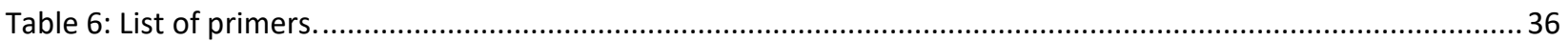

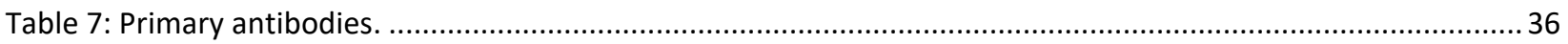

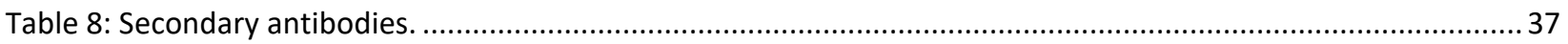

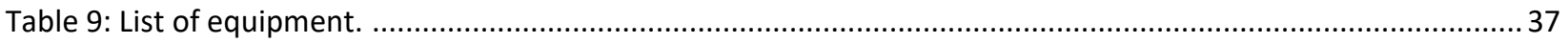

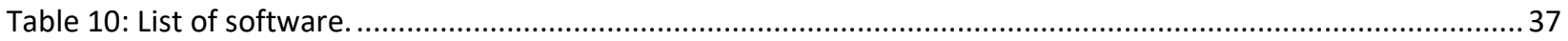

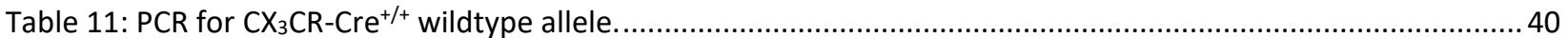

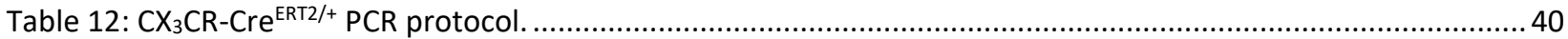

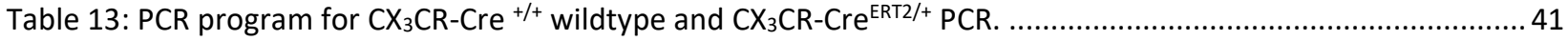

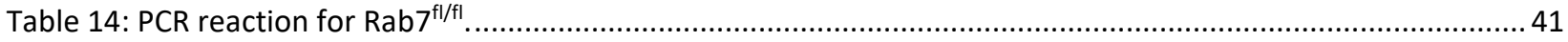

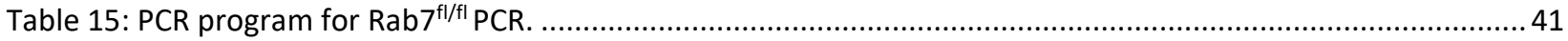

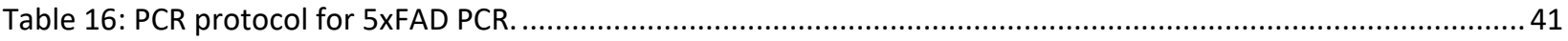

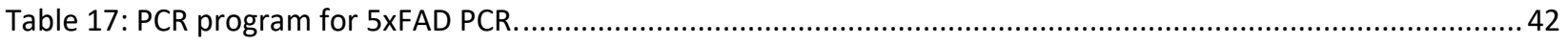

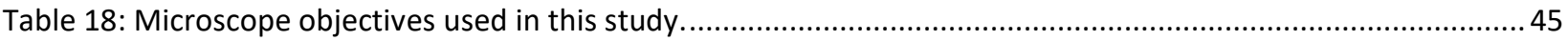

Table 19: Protocol for one SDS gel composed of $12 \%$ resolving gel and $4 \%$ stacking gel....................................51 


\section{Abbreviations}

\begin{tabular}{|c|c|}
\hline Abbreviation & Meaning \\
\hline+ & plus or positive \\
\hline${ }^{\circ} \mathrm{C}$ & degree celsius \\
\hline$\mu$ & micro $(10 \mathrm{e}-6)$ \\
\hline $5 x F A D$ & five times familial Alzheimer's disease \\
\hline $6 \mathrm{E} 10$ & $n$-terminal antibody against $A \beta$ \\
\hline aa & amino acids \\
\hline$A D$ & Alzheimer's disease \\
\hline APOE & Apolipoprotein E \\
\hline APP & Amyloid precursor protein \\
\hline$A \beta$ & $\beta$-amyloid peptide \\
\hline $\mathrm{BCA}$ & bicinchoninic acid assay \\
\hline bp & base pair \\
\hline BSA & bovine serum albumin \\
\hline CD68 & Cluster differentiation 68; or Lysosome-associated membrane protein 4, LAMP4 \\
\hline CHAPS & 3-[(3-cholamidopropyl)dimethylammonio]-1-propanesulfonate \\
\hline CNS & central nervous system \\
\hline $\mathrm{CX}_{3} \mathrm{CR} 1$ & CX3C chemokine receptor 1 \\
\hline DABCO & 1,4-Diazabicyclo[2.2.2]octan \\
\hline DAPI & 4',6-diamidino-2-phenylindole \\
\hline $\mathrm{ddH}_{2} \mathrm{O}$ & double-distilled water \\
\hline DNA & desoxyribonuclein acid \\
\hline ECL & electrochemiluminescence \\
\hline EDTA & ethylenediaminetetraacetic acid \\
\hline et al. & et alii (Latin = and others) \\
\hline FAD & familial Alzheimer's disease \\
\hline FCS & fetal calf serum \\
\hline $\mathrm{fl}$ & lox P site \\
\hline g & gram \\
\hline GFAP & Glial fibrillary acidic protein \\
\hline $\mathrm{h}$ & hour \\
\hline HBSS & Hank`s buffered saline solution \\
\hline HRP & horseradish peroxidase \\
\hline i.p. & intraperitoneal \\
\hline lba1 & Ionized calcium-binding adapter molecule 1 \\
\hline
\end{tabular}




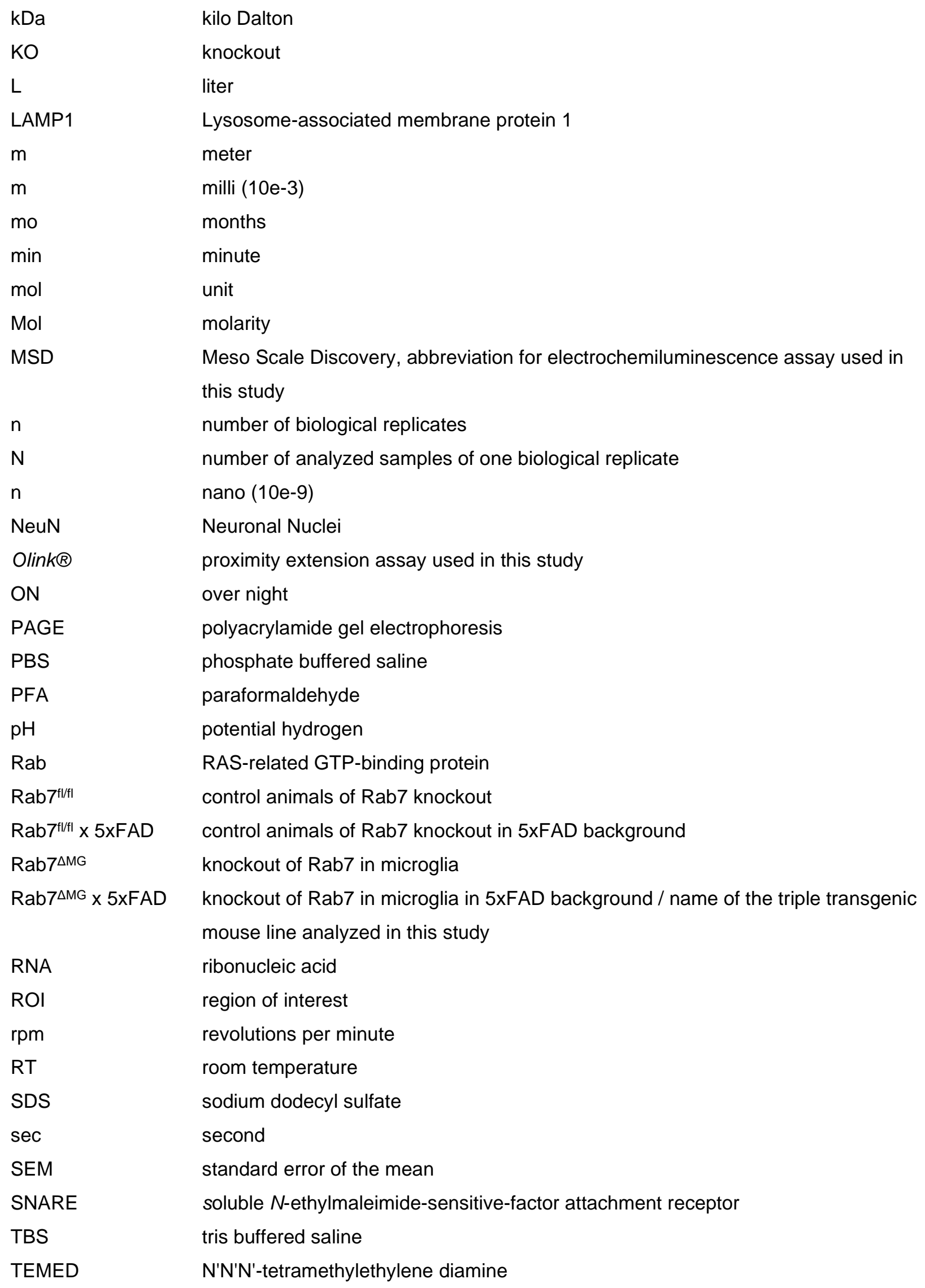


Abbreviations

TREM2

Tris- $\mathrm{HCl}$

$\mathrm{v} / \mathrm{v}$

$\mathrm{w} / \mathrm{v}$

WB

WT

x $g$
Triggering receptor expressed on myeloid cells 2

tris(hydroxymethyl)aminomethane-hydroxyl chloride

volume per volume

weight per volume

Western Blot

wildtype

times gravitational acceleration 


\section{Abbreviations of proteins analyzed by Olink $®$ proximity ligation assay}

\begin{tabular}{|c|c|}
\hline Abbreviation & Protein name \\
\hline Acvrl1 & Serine/threonine-protein kinase receptor R3 \\
\hline Adam23 & Disintegrin and metalloproteinase domain-containing protein 23 \\
\hline Ahr & Aryl hydrocarbon receptor \\
\hline Apbb1ip & Amyloid beta $A 4$ precursor protein-binding family $B$ member 1-interacting protein \\
\hline Axin1 & Axin-1 \\
\hline Ca13 & Carbonic anhydrase 13 \\
\hline Cant1 & Soluble calcium-activated nucleotidase 1 \\
\hline Casp3 & Caspase-3 \\
\hline Ccl2 & C-C motif chemokine 2 \\
\hline Ccl20 & C-C motif chemokine 20 \\
\hline Ccl3 & C-C motif chemokine 3 \\
\hline Ccl5 & C-C motif chemokine 5 \\
\hline Cdh6 & Cadherin-6 - mouse \\
\hline Clmp & CXADR-like membrane protein \\
\hline Clstn2 & Calsyntenin-2 \\
\hline Cntn1 & Contactin-1 \\
\hline Cntn4 & Contactin-4 \\
\hline Cpe & Carboxypeptidase E \\
\hline Crim1 & Cysteine-rich motor neuron 1 protein \\
\hline Csf2 & Granulocyte-macrophage colony-stimulating factor \\
\hline Cxcl1 & Growth-regulated alpha protein \\
\hline Cxcl9 & C-X-C motif chemokine 9 \\
\hline Cyr61 & Protein CYR61 \\
\hline Dctn2 & Dynactin subunit 2 \\
\hline Ddah1 & $\mathrm{N}(\mathrm{G}), \mathrm{N}(\mathrm{G})$-dimethylarginine dimethylaminohydrolase 1 \\
\hline Dlk1 & Protein delta homolog 1 \\
\hline DII1 & Delta-like protein 1 \\
\hline Eda2r & Tumor necrosis factor receptor superfamily member 27 \\
\hline Eno2 & Gamma-enolase \\
\hline Epcam & Epithelial cell adhesion molecule \\
\hline Epo & Erythropoietin \\
\hline Erbb4 & Receptor tyrosine-protein kinase erbB-4 \\
\hline
\end{tabular}




\begin{tabular}{|c|c|}
\hline Fas & Tumor necrosis factor receptor superfamily member 6 \\
\hline Fli1 & Friend leukemia integration 1 transcription factor \\
\hline Flrt2 & Leucine-rich repeat transmembrane protein \\
\hline Foxo1 & Forkhead box protein $\mathrm{O} 1$ \\
\hline Fst & Follistatin \\
\hline Fstl3 & Follistatin-related protein 3 \\
\hline Gcg & Glucagon \\
\hline Gdnf & Glial cell line-derived neurotrophic factor \\
\hline Gfra1 & GDNF family receptor alpha-1 \\
\hline Ghrl & Appetite-regulating hormone \\
\hline Hgf & Hepatocyte growth factor \\
\hline lgsf3 & Immunoglobulin superfamily member 3 \\
\hline$\| 10$ & Interleukin-10 \\
\hline $1117 \mathrm{a}$ & Interleukin-17A \\
\hline$\| 17 f$ & Interleukin-17F \\
\hline$\| 1 \alpha$ & Interleukin-1 alpha \\
\hline$\| 1 \beta$ & Interleukin-1 beta \\
\hline$\| 23 r$ & Interleukin-23 receptor \\
\hline 115 & Interleukin-5 \\
\hline 116 & Interleukin-6 \\
\hline $\operatorname{ltg} \beta 1 \mathrm{bp} 2$ & Integrin beta-1-binding protein 2 \\
\hline $\operatorname{ltg} \beta 6$ & Integrin beta- 6 \\
\hline Kitlg & Kit ligand \\
\hline Lgmn & Legumain \\
\hline Lpl & Lipoprotein lipase \\
\hline Map2k6 & Dual specificity mitogen-activated protein kinase kinase 6 \\
\hline Matn2 & Matrilin-2 \\
\hline Mia & Melanoma-derived growth regulatory protein \\
\hline Nadk & NAD kinase \\
\hline Notch3 & Neurogenic locus notch homolog protein 3 \\
\hline Ntf3 & Neurotrophin-3 \\
\hline Pak4 & Serine/threonine-protein kinase PAK 4 \\
\hline Parp1 & Poly [ADP-ribose] polymerase 1 \\
\hline Pdgfb & Platelet-derived growth factor subunit B \\
\hline Pla2g4a & Cytosolic phospholipase A2 \\
\hline Plin1 & Perilipin-1 \\
\hline Plxna4 & Plexin-A4 \\
\hline Ppp1r2 & Protein phosphatase inhibitor 2 \\
\hline
\end{tabular}




$\begin{array}{ll}\text { Prdx5 } & \text { Peroxiredoxin-5 } \\ \text { Qdpr } & \text { Dihydropteridine reductase } \\ \text { Rgma } & \text { Repulsive guidance molecule A } \\ \text { Riox2 } & \text { Ribosomal oxygenase 2 } \\ \text { S100a4 } & \text { Protein S100-A4 } \\ \text { Sez6l2 } & \text { Seizure 6-like protein 2 } \\ \text { Snap29 } & \text { Synaptosomal-associated protein 29 } \\ \text { Tgfa } & \text { Protransforming growth factor alpha } \\ \text { Tgfß1 } & \text { Latency-associated peptide transforming growth factor beta-1 } \\ \text { Tgfßr3 } & \text { Transforming growth factor beta receptor type 3 } \\ \text { Tnf } & \text { Tumor necrosis factor } \\ \text { Tnfrsf11b } & \text { Tumor necrosis factor receptor superfamily member 11B } \\ \text { Tnfrsf12a } & \text { Tumor necrosis factor receptor superfamily member 12A } \\ \text { Tnfsf12 } & \text { Tumor necrosis factor ligand superfamily member 12 } \\ \text { Tnni3 } & \text { Troponin I } \\ \text { Tnr } & \text { Tenascin-R } \\ \text { Tpp1 } & \text { Tripeptidyl-peptidase 1 } \\ \text { Vegfd } & \text { Vascular endothelial growth factor D } \\ \text { Vsig2 } & \text { V-set and immunoglobulin domain-containing protein 2 } \\ \text { Wfikkn2 } & \text { WAP, Kazal, immunoglobulin, Kunitz and NTR domain-containing protein 2 } \\ \text { Wisp1 } & \text { WNT1-inducible-signaling pathway protein 1 } \\ \text { Yes1 } & \text { Tyrosine-protein kinase Yes } \\ & \end{array}$




\section{Acknowledgements}

First of all, I would like to thank my supervisor Prof. Dr. Anja Schneider for the opportunity of working on several interesting projects and for supporting me throughout my $\mathrm{PhD}$. I am deeply grateful for your scientific guidance, fruitful discussions and your input and support throughout these past four years. You always took the time to supervise me - even across the ocean and during the time of our lab transition to Bonn. You helped me to progress and further develop as a scientist and as a person. Therefore, I would like to express my sincere gratitude.

I would like to thank Prof. Dr. Silvio Rizzoli and Prof. Dr. André Fischer for being part of my thesis committee and for their valuable advises and helpful discussions. In particular, I would like to address my gratitude towards the fantastic support for the times outside of Göttingen! Furthermore, I would like to thank Prof. Dr. Mikael Simons, Prof. Dr. Dr. Hannelore Ehrenreich and Prof. Dr. Alexander Flügel for their willingness to be members of the extended examination board and for taking the time to consider my work.

I am also grateful for the possibility of graduating within the Göttingen Graduate School for Neuroscience and Biophysics (GGNB), which provided a great learning environment by organizing interesting PhD seminars and retreats. In particular, I am grateful to Prof. Dr. Michael Hörner for his perpetual support.

Moreover, I would like to address my deepest gratitude to Prof. Dr. Tony Wyss-Coray and his lab for the pleasure and great opportunity of working on an exciting cooperation project about brain aging for several months.

I would also like to thank Prof. Dr. Mikael Simons, for hosting me in his lab during the transition phase of our lab to Bonn.

Also, I am very thankful to everyone who contributed to this project: Angela Dettmer, Dr. Ana MartinezHernandez, Michaela Limmer, Pascal Kalben and Angeliki Spathopoulou.

I would like to thank Dr. Manuel Schöllinger for the Image and Data Analysis Facility (IDAF), Bonn, for creating the plugin for region-specific analysis within Imaris. Also, I appreciate the technical support of the Light Microscope Facility (LMF), Bonn. Moreover, I would like to thank the animal caretakers of MPI-BC and ENI for their great work contributing to this project!

My sincere acknowledgements also go out to everyone who has helped me in the past few weeks finishing up this thesis, by giving great advices, by proof-reading or by just offering me a cup of coffee and some time to talk about something not science-related ;-). This includes in particular Anna Antoniou, 
Elisabeth Berber, Cira Dansokho, Eva Kess, Tanja Kraus, Stephanie Lohmann, Julia Marschallinger, Karishma Mistry, Andreas Wild and Stefanie Zentelis.

My gratitude also goes out to all current and former members of the Schneider lab for the great working atmosphere:

- Anna: thanks for the frequent chats and random yoga classes together

- Anne: thanks for the wonderful times inside and outside the lab including Christmas cookie baking, picnics and car-pooling to Göttingen :)

- Belisa: even though I still don't understand Portuguese, I truly appreciate that you've introduced me to pastéis de nata and I am deeply grateful for our friendship :)

- Björn: as original cheerful Rhenish soul ("rheinische Frohnatur") you contributed to my decreased skepticism of carnival ;-)

- Eva: thanks for Zumba-summer and your positive attitude

- Jing: thanks for your cheerfulness

- Marcel: thanks for the funny moments before, throughout and after the lab moving ;-)

- Tanja: thanks for the wonderful working sessions on the weekend and fantastic moments outside of the lab :-

I would like to thank Elisabeth, Markus, Julia, Izumi, Karishma, Betty and Huy. You guys were and always will be my California family!

Also, I would like to thank everyone from the E-block, in particular Bárbara, Ludo, Maryam and Uli, who adopted me during the lab transition from Göttingen to Bonn. Thanks for always being around when I needed equipment, advice or, most importantly, a coffee break ;-).

Adri, Meike, Joi, Kerstin and Melli, you made Göttingen a second home for me! Thank you for your friendship!

Zu guter Letzte möchte ich noch meiner Familie meinen tiefsten Dank aussprechen. Danke für Eure liebevolle Unterstützung und Euren unerschütterlichen Glauben an mich. Danke, dass Ihr immer für mich da wart und seid und mich bei all meinen Entscheidungen unterstützt! 


\section{Abstract}

Microglia are the innate immune cells of the brain. In neurodegeneration, such as Alzheimer's disease, these cells are involved in clearance of cellular debris and extracellular aggregates of misfolded proteins like amyloid beta $(A \beta)$. However, alongside $A \beta$-clearance microglia are activated, resulting amongst others in the secretion of neurotoxic cytokines which contributes to neuronal cell death. Hence, it is not well understood whether microglia are beneficial or detrimental for the progression of AD. To address this question, we generated 5xFAD transgenic AD mice with microglia-specific depletion of Rab7 GTPase $\left(R a b 7{ }^{\triangle M G} \times 5 \times F A D\right)$, which is responsible for the fusion of late endosomes and autophagosomes with the lysosome, and thereby promotes lysosomal degradation.

Surprisingly, we found that Rab7 ${ }^{\triangle M G} \times 5 \times F A D$ mice revealed fewer and smaller $A \beta$-plaques in aged female mice, while the overall amounts of soluble and insoluble $A \beta$-species were not changed in early ( 3 months) and progressed (9 months) stages of disease. We did not observe changes in micro- and astrogliosis. However, elevated levels of CCL2 were detected, which could provide a mechanism to attract peripheral derived myeloid cells which could compensate for degradation impaired Rab7 knockout microglia.

All of these findings were exclusively found in female Rab7 ${ }^{\triangle M G} \times 5 \times F A D$ mice. This could either be caused by higher basal $A \beta$-burden in females or by sex-dependent differential features of microglia, including phago-lysosomal activity. 


\section{Table of Contents}

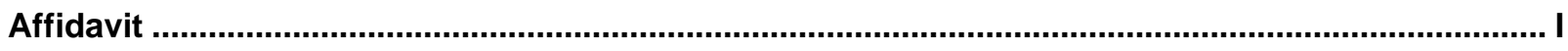

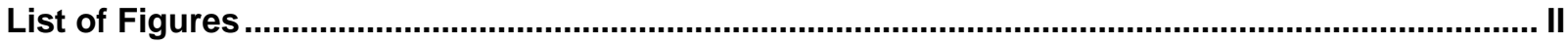

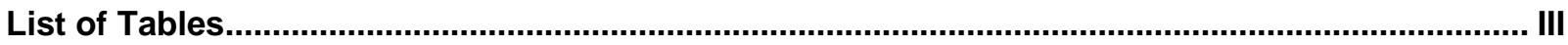

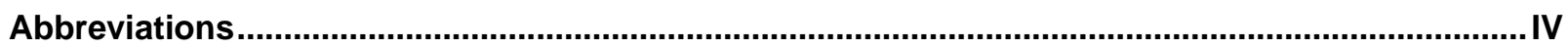

Abbreviations of proteins analyzed by Olink ${ }^{\circledR}$ proximity ligation assay .......................................... VII

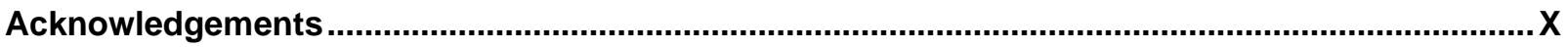

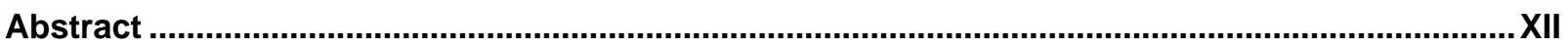

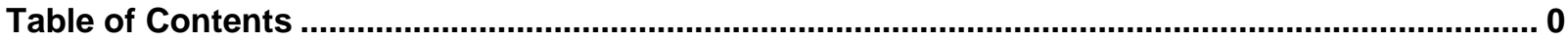

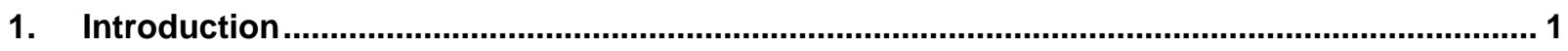

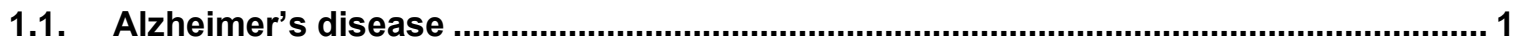

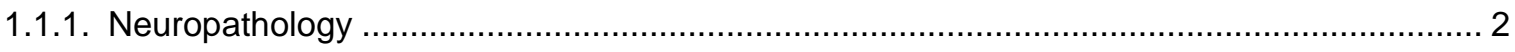

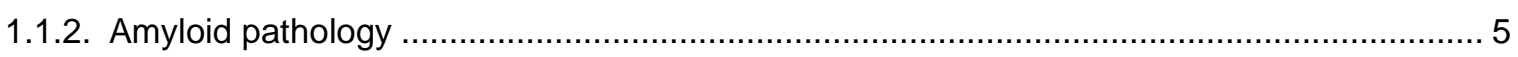

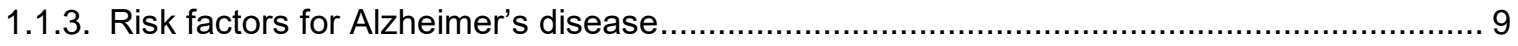

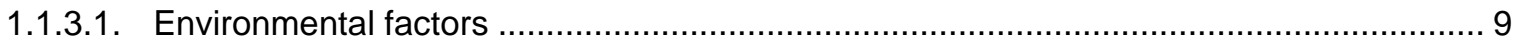

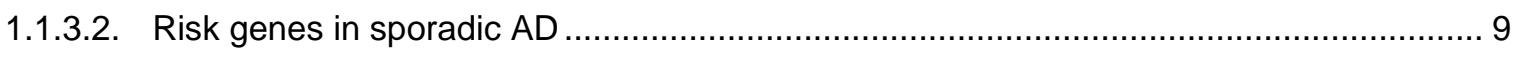

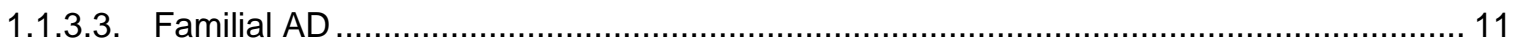

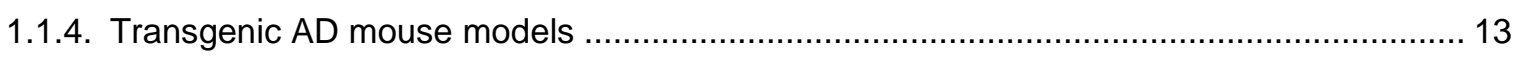

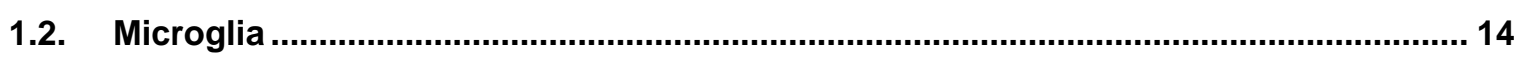

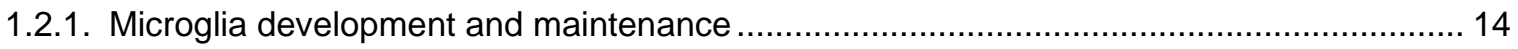

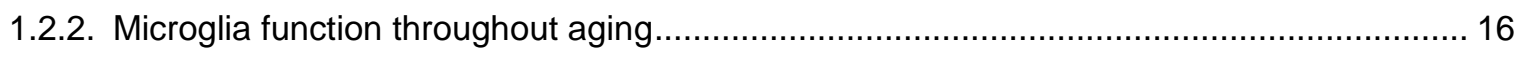

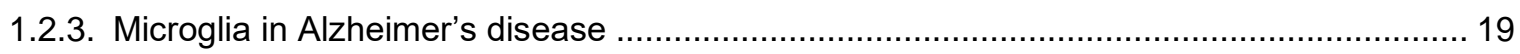

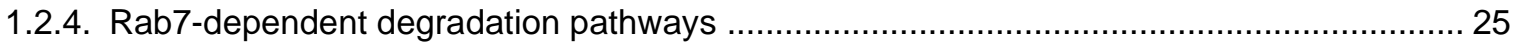

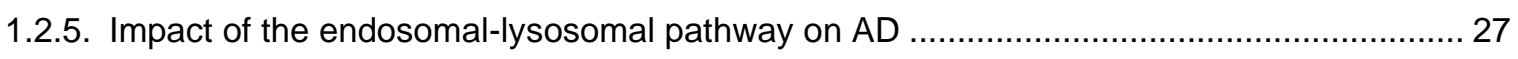

1.2.6. Targeting microglial endo-lysosomal degradation in vivo: the Rab7 $\triangle \mathrm{MG}$ mouse line ........... 29

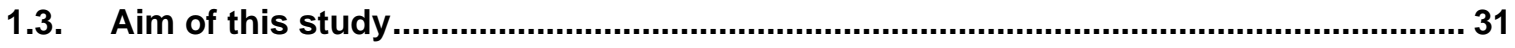

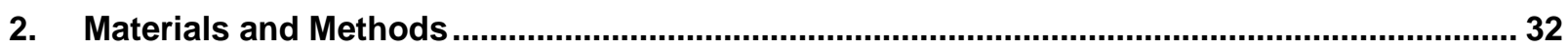

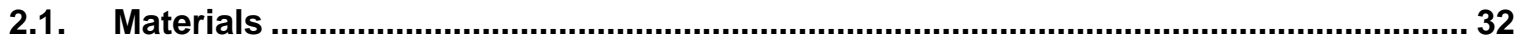

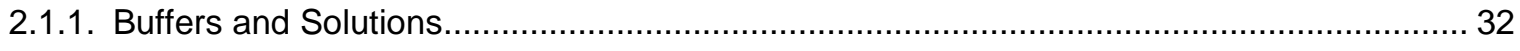

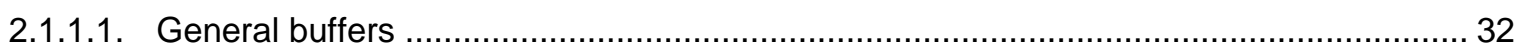

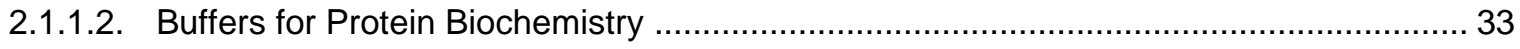

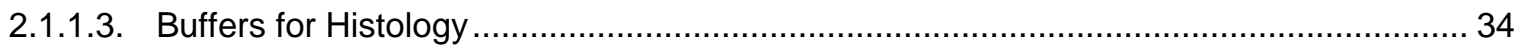

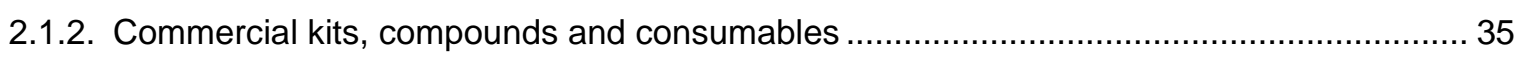

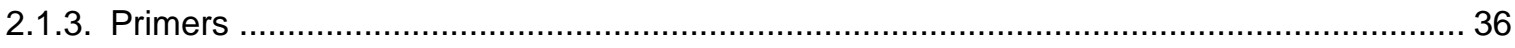

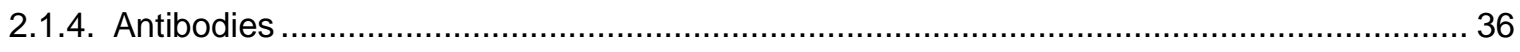

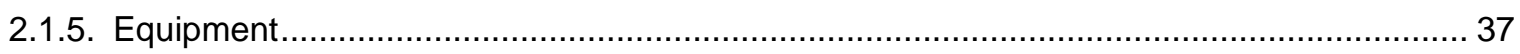

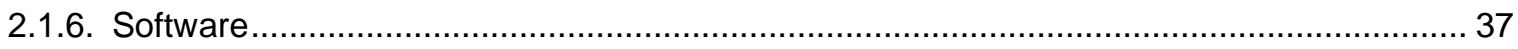

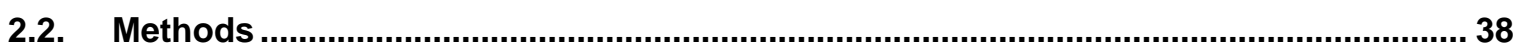




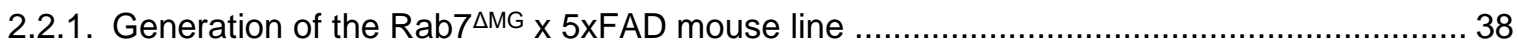

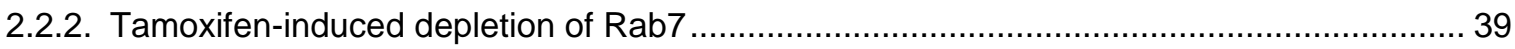

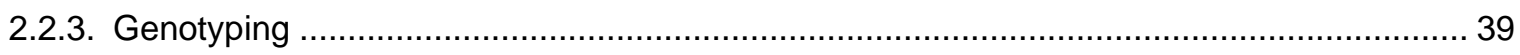

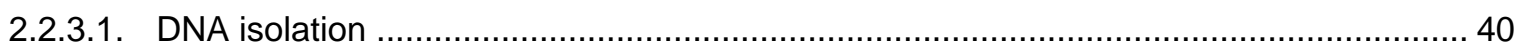

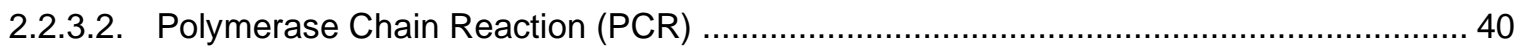

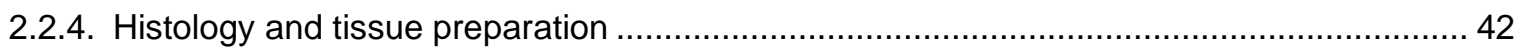

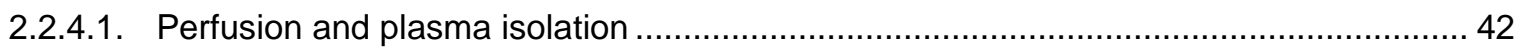

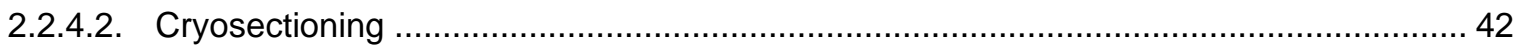

2.2.4.3. Free-floating immunohistochemistry with fluorescence detection ............................... 42

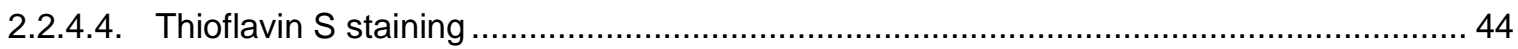

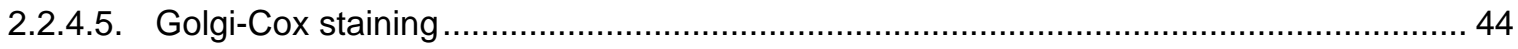

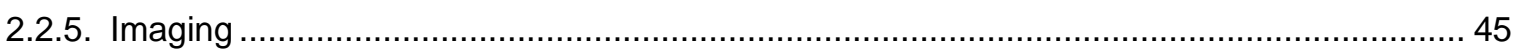

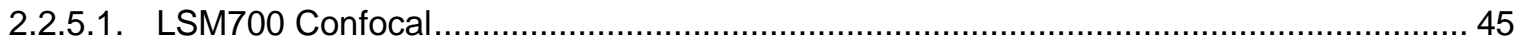

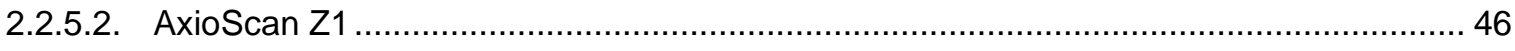

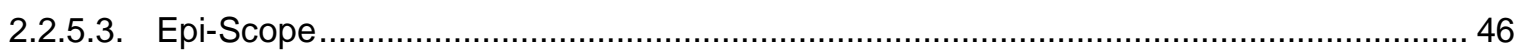

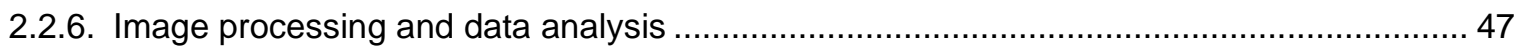

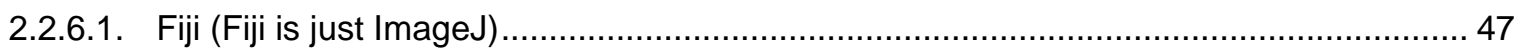

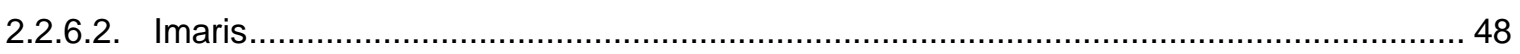

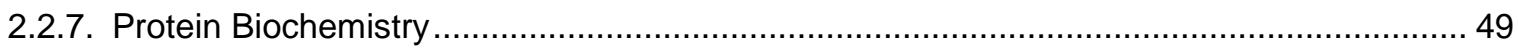

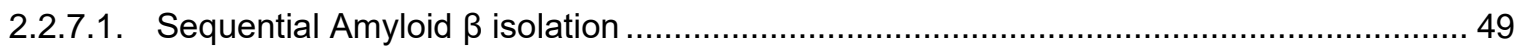

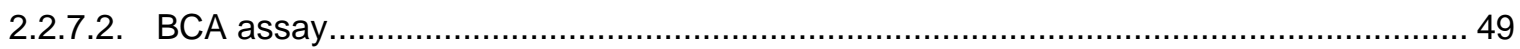

2.2.7.3. Meso Scale Discovery ${ }^{\mathrm{TM}}$ (MSD) Electrochemi-luminescence ..................................... 50

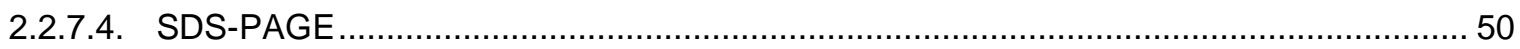

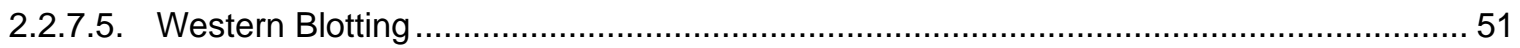

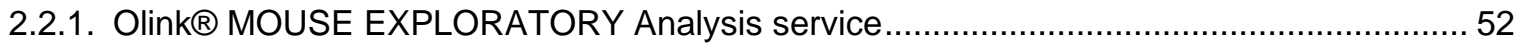

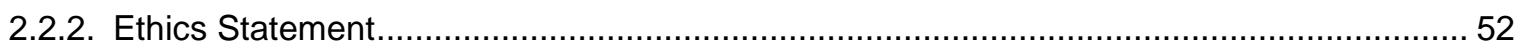

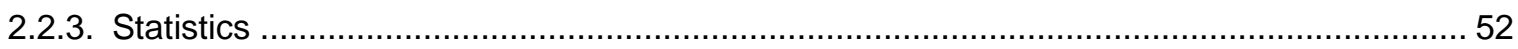

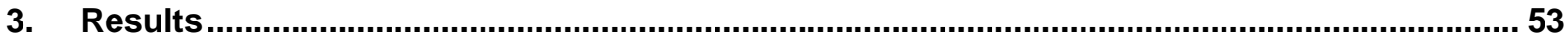

3.1. Generation and validation of the Rab7 ${ }^{\mathrm{MMG}} \times 5 \times$ XAD mouse model ............................53

3.1.1. Microglia of Rab7 ${ }^{\triangle M G} \times 5 \times F A D$ mice showed increased CD68 positive endo-lysosomes ... 54

3.1.2. Enlargement of $L A M P 1$ positive lysosomes were found in Rab7 ${ }^{\triangle M G} \times 5 \times F A D$ mice ........... 55

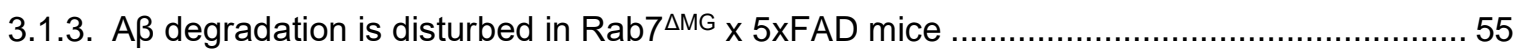

3.2. Plaque pathology in the Rab7 ${ }^{\triangle M G} \times 5 x F A D$ mice was reduced .................................. 56

3.2.1. Microglial Rab7 $\triangle M G$ reduces $A \beta$-plaque numbers throughout disease progression in

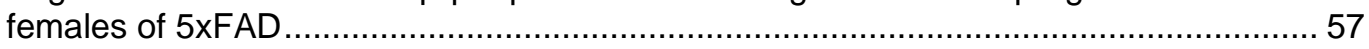

3.2.2. A $A$-plaques were altered in morphology and composition ............................................. 59

3.2.3. No differences in concentrations of soluble and insoluble $A \beta$-peptides were found in

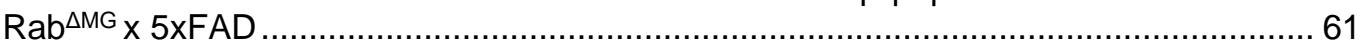

3.3. Neuronal, dendritic spine and synaptic loss were not altered in the Rab7 ${ }^{\Delta \mathrm{MG}} \mathrm{x}$ 5xFAD mice

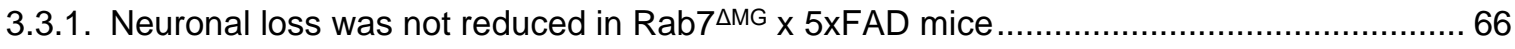


3.3.2. $R a b 7^{\triangle M G} \times 5 \times F A D$ showed mild effects on dendritic spine loss 67

3.3.3. Synaptic loss was not altered due to Rab7 KO 68

3.4. Phenotypical characterization of microglia in the Rab7 $7^{\Delta M G} \times 5 \times F A D$ model. 69

3.4.1. Rab7 $\triangle M G G \times 5 \times F A D$ had no effects on absolute microglia numbers or on their recruitment .69

3.4.2. Rab7 ${ }^{\triangle M G} \times 5 \times F A D$ did not display altered cellular morphology of microglia ........................ 73

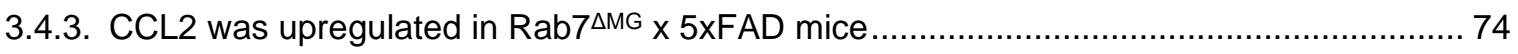

3.5. Rab7 ${ }^{\triangle \mathrm{MG}}$ cannot be compensated by other brain cells in the $5 x F A D$ mouse model .. 76

3.5.1. Astrogliosis was not affected in Rab7 ${ }^{\triangle M G} \times 5 \times F A D$ mice. .76

3.5.2. No compensation of $A \beta$-degradation by astrocytes was found in Rab7 $\triangle M G \times 5 \times F A D$

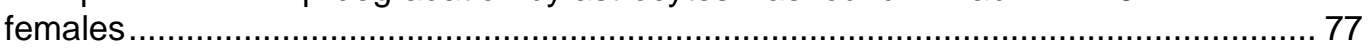

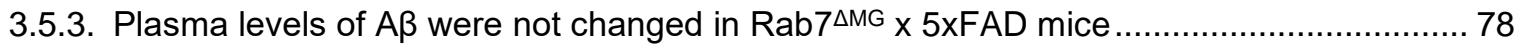

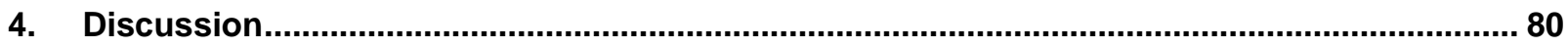

4.1. Evaluation of the microglial Rab7 KO in 5xFAD mice............................................... 80

4.2. Compensatory mechanisms for Rab7 KO in microglia ................................................ 81

4.3. Effects of microglial Rab7 KO on amyloid-pathology and neurotoxicity in 5xFAD mice

4.4. Role of sex on Aß-load in Rab7-mediated degradation in microglia ......................... 89

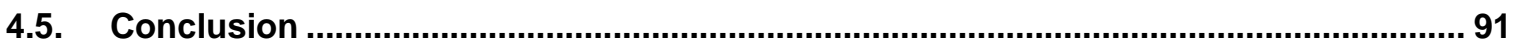

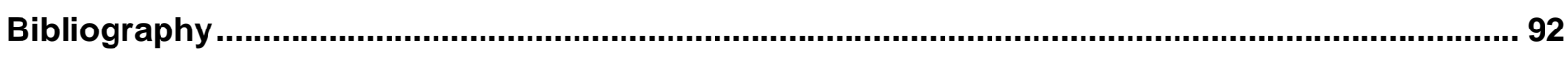

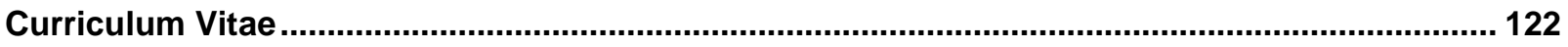




\section{Introduction}

\subsection{Alzheimer's disease}

Alzheimer's disease (AD) is named after the psychiatrist and neuropathologist Alois Alzheimer who first described the pathology in 1907 (Alzheimer, 1907; English translation: Alzheimer et al., 1995). As of today, $A D$ is the most common form of dementia in the elderly with aging as a major risk factor. Its prevalence increases due to higher life-expectancies. In 2035 about 90 million are predicted to be diagnosed with AD worldwide (Jahn, 2013; Weuve et al., 2014). The disease is characterized by the progressive and irreversible loss of memory and other cognitive functions as well as by changes in behavior and mood due to neuronal and synaptic loss in the brain (Lam et al., 2013).

\section{Symptoms and Diagnosis}

The neuropathology underlying $A D$ begins two to three decades before the first clinical symptoms occur (Masters et al., 2015). This period is called preclinical phase. Neuropathological processes increase over time, resulting in early symptoms, e.g. deficits in short term memory, which do not fulfill the criteria of dementia. This stage is termed mild cognitive impairment $(\mathrm{MCl})$ or prodromal disease stage. In the dementia stage, patients experience a progressive loss of spatial orientation and language skills, with additional impairment of daily living functions. Additionally, many patients experience changes in their personality and behavior, e.g. aggression or delusion (Dubois et al., 2007; Galvin et al., 2010).

The diagnosis of $A D$ relies on a combination of neuropsychological testing, structural and functional MRI (Magnetic Resonance Imaging), FDG-PET (Fluorodeoxyglucose Positron Emission Tomography) and amyloid PET, and cerebrospinal fluid (CSF) biomarker analysis. AD diagnosis can only be secured post mortem by detection of amyloid plaques and neurofibrillary tangles with immunohistochemistry (Reitz and Mayeux, 2014).

\section{Therapy}

As of today, there is no cure for AD. So far, only two different symptomatic therapies have been approved for treatment of AD, acetylcholinesterase inhibitors and memantine (Glynn-Servedio and Ranola, 2017).

In the early 1980s Whitehouse and colleagues discovered that cholinergic neurons were affected in brains of $A D$ patients. Thus, treatment with acetylcholinestrase inhibitors is currently a therapy for early stage and moderate AD. The inhibitor blocks the enzymatic inactivation of acetylcholine through acetylcholinesterases in the synaptic cleft resulting in higher concentrations of this neurotransmitter as compensation for the loss of cholinergic neurons (Frozza et al., 2018). At 
moderate and severe stages of $A D$, the $\mathrm{N}$-methyl-aspartate (NMDA)-receptor antagonist memantine improves daily living functions in AD patients. Throughout the disease, NMDA-receptors tend to be over-stimulated, which can be counteracted by memantine (Frozza et al., 2018).

\section{Epidemiology}

Most cases of $A D$ are diagnosed at 65 years or older (Masters et al., 2015). A minority of around $1 \%$ of $A D$ is caused by familial autosomal dominant mutations and the majority of cases are sporadic (see section 1.1.3). In the United States of America about two-third of diagnosed AD patients are women with this sex-discrepancy being based on higher life expectancies of women (Hebert et al., 2001). However, a well described risk factor for AD development, namely Apoprotein ApoE4, was found to have stronger association with $A D$ in women than in men between 65 and 75 years of age (Altmann et al., 2014; Farrer et al., 1997; Neu et al., 2017). There is evidence suggesting interaction of the sex hormone estrogen and ApoE4, which could explain these sexdiscrepancies about 10 years post-menopause in women (Kang and Grodstein, 2012; Neu et al., 2017).

\subsubsection{Neuropathology}

The histopathology of $A D$ consists of four prominent hallmarks: senile amyloid $(A \beta)$ plaques, neurofibrillary tangles (NFTs), brain atrophy and neuroinflammation (Figure 1).

\section{Senile $A \beta$-plaques}

Senile plaques were identified as extracellular aggregates of $A \beta$-peptides (Masters et al., 1985). $A \beta$ is cleaved from the highly conserved integral membrane protein Amyloid Precursor Protein (APP), which is encoded by the APP gene located on chromosome 21 in humans. APP contains 18 exons with a total length of $290 \mathrm{~kb}$ (kilobases) (Yoshikai et al., 1990). Various splicing variants of APP can be found in different tissues and cell types in mammals. In human neurons the splicing variant APP695 is the most abundant one (reviewed by Matsui et al., 2007). Noteworthy, APP is extensively post-translationally modified. This includes amongst others glycosylation, sialylation and phosphorylation but also enzymatic processing (Kummer and Heneka, 2014). Enzymatic processing of APP can occur e.g. in an amyloidogenic or non-amyloidogenic manner, which will be described in detail in section 1.1.2.

$A \beta$-plaques can be categorized in dense-core also known as neuritic plaques and in diffuse plaques (Wisniewski et al., 1973). Dense-core $A \beta$-plaques consist of fibrillary amyloid clustering in a central core surrounded by loose $A \beta$-peptides, dystrophic neurites and gliosis. The dense core can be visualized by $\beta$-sheet binding dyes like Congo Red, Methoxy-XO4 or ThioflavinS (ThioS). Diffuse plaques are more amorphous lacking dystrophic neurites and a central core. Thus, they cannot be labeled with $\beta$-sheet binding dyes and need to be visualized by antibody staining (Selkoe, 2001). 

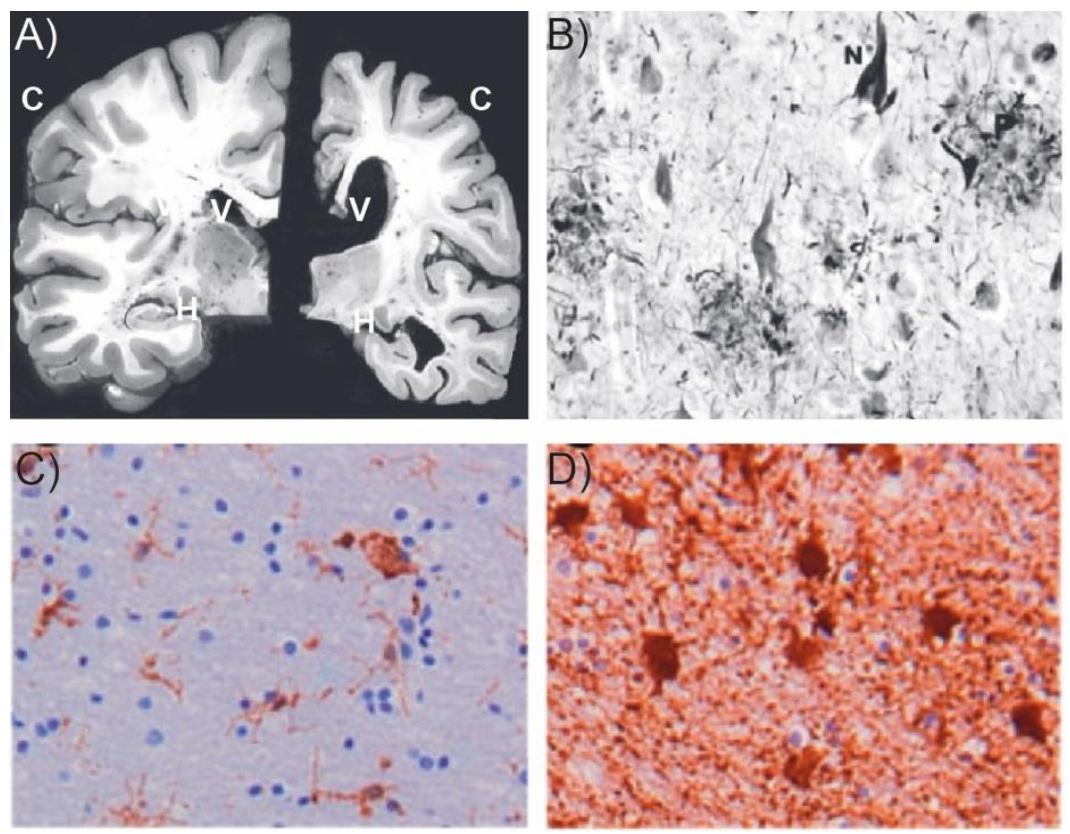

Figure 1: Histopathological hallmarks of $A D$.

A) Atrophic hemibrain of a 70 year old $A D$ patient (right) in comparison to a hemibrain of an age-matched healthy control (left). The cortex $(C)$ shows extreme shriveling in the AD brain compared to the control brain. The hippocampus $(\mathrm{H})$ also displays massive shrinkage, while the lateral ventricle $(\mathrm{V})$ is prominently increased in the AD sample. B) Silver staining on post mortem brain tissue of the $A D$ patient shows neuritic plaques $(P)$ and neurofibrillary tangles $(N)$. C) Immunohistochemistry for MHC II (major histocompatibility complex II) in brown labels reactive microglia in AD post mortem tissue. D) Alongside astrogliosis is detcted by staining for GFAP (glial fibrillary acidic protein) in brown. C+D) Blue counterstaining with haematoxylin labels nuclei. Images were modified after Gouw et al., (2008) and Wippold et al., (2008).

Spreading of $A \beta$-plaques occurs in a distinct pattern, which can be categorized by the Thal $A \beta$ phase (TAP) or the Consortium to Establish a Registry for Alzheimer's Disease (CERAD) system (Mirra et al., 1991; Thal et al., 2002). The TAP system describes anatomical distribution of $A \beta$-plaques beginning in the neocortex (TAP 1), proceeding in the hippocampus, amygdala, allocortex and diencephalon (TAP 2+3) and further appears in the brain stem and cerebellum (TAP $4+5$ ) according to immunohistochemical analysis (Thal et al., 2002) (Figure 2A). CERAD uses a semi-quantitative approach to assess neuritic plaques, ranging from none (0), sparse (1), moderate (2) to severe (3) deposition (Mirra et al., 1991).

\section{Neurofibrillary tangles}

Intraneuronal protein accumulations known as NFTs are found in post mortem tissue of AD patients. NFTs are built of paired helical filaments (PHFs), which consist of hyperphosphorylated Tau protein (pTau), a microtubule-associated protein (MAPT) (Goedert et al., 1988; Kidd, 1963; Kopke et al., 1993). Under physiological conditions Tau is a modulator of the microtubule assembly and stabilization as well as of axonal transport (Goedert et al., 2006; Weingarten et al., 1975). Upon 
hyperphosphorylation Tau becomes dysfunctional, which is associated with synaptic dysfunction, altered intracellular trafficking and defective proteasomal degradation (Wang and Mandelkow, 2016).

Neither the pathological mechanisms causing Tau hyperphosphorylation, nor its interaction with $A \beta$ peptides are fully understood yet (Hochgrafe et al., 2013; Sydow et al., 2011). A $\beta$-pathology seems to be upstream of Tau pathology as studies using Tau knockout $(\mathrm{KO})$ mice with overexpression of human APP found neuroprotection even though A $\beta$-burden was not altered (Roberson et al., 2007). Furthermore, $A \beta$ oligomerization can trigger $\mathrm{pTau}$ accumulation in neurons and thereby promote NTF formation (Ma et al., 2009; Oddo et al., 2003; Zempel et al., 2013).

Albeit NFTs are a hallmark of $A D$ and mutations in the human MAPT gene are associated with genetic forms of frontotemporal dementia and other tauopathies, there is no genetic link found to AD (Wolfe, 2009).

In contrast to $A \beta$ pathology, the location of NFTs correlates closely with the severity of $A D$. Therefore, spreading pattern of NFTs are staged according to the so-called Braak stages (Braak and Braak, 1991). Stage I and II describe NFTs in the locus coeruleus, the entorhinal and transentorhinal regions (Figure 2B). Stages III and IV describe NFTs in the hippocampus and within the frontal parts of the neocortex. NFT-burden in further parts of the neocortex is staged with Braak stages $\mathrm{V}$ and VI.

\section{Brain atrophy}

Brain atrophy is a very prominent feature of AD brains, correlating with NFT-burden and reflecting neuronal loss. Neuronal loss results in atrophy of hippocampus, temporal lobes and eventually

A)

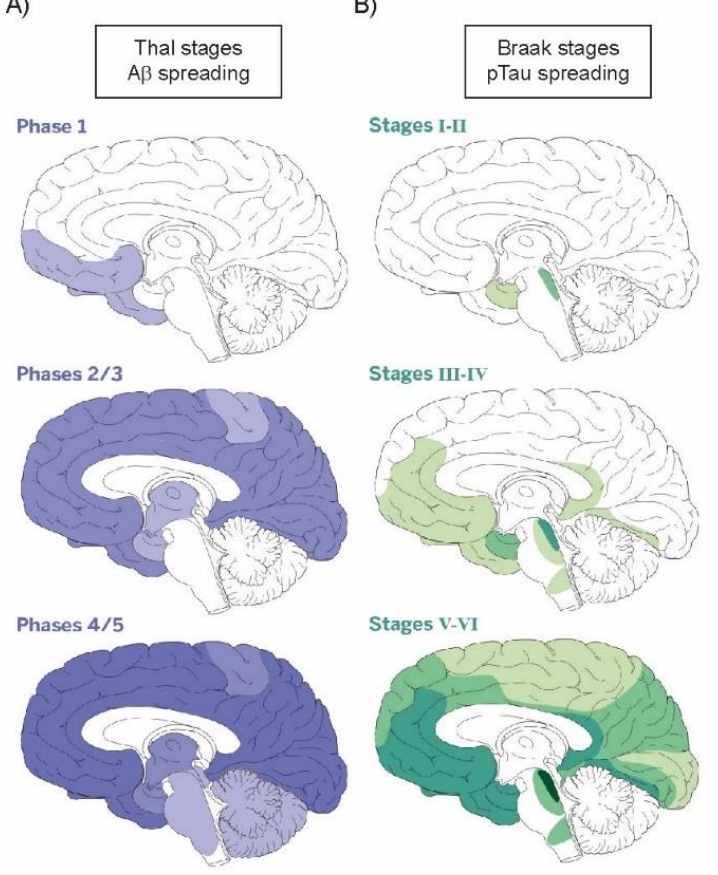

Figure 2: Thal stages of amyloid $(A \beta)$ and Braak stages of NFT pathology.

A) Thal stages of $A \beta$-plaque pathology are shown in blue. Phase 1 describes $A \beta$-deposits in the basal temporal cortex and in the orbitofrontal neocortex. Phases 2 and 3 classify $A \beta$ throughout the neocortex, in the hippocampus, the amygdala, the basal ganglia and the diencephalon. Phases 4 and 5 are used to describe $A \beta$-deposits in the mesencephalon, cerebral cortex and the lower brainstem. B) NFT pathology is shown in green. Stage I and II describes intraneuronal accumulations of hyperphophorylated Tau in the loculs coeruleus, the entorhinal and transentorhinal cortex. Stage III and IV are used when NFTs are detected in the hippocampus and in the frontal neocortex. Stages $\mathrm{V}$ and VI define NFTs in neurons throughout the neocortex. Figure adapted from Goedert et al. (2015). 
parietal cortex. Furthermore, it causes enlargement of ventricles (Figure 1A). Progressive reduction of brain volume due to neuronal loss can already be detected at early stages of the disease by MRI (Leung et al., 2013). Neuronal loss is preceded by synaptic dysfunction. All of these aspects lead to memory impairment.

\section{Neuroinflammation}

Another important finding in post mortem brains of $A D$ patients is neuroinflammation. The term neuroinflammation describes activation of immune cells in the CNS as a consequence of brain injury, trauma or infection. Neuroinflammation is accompanied by reactive gliosis, which describes activation and proliferation of glia. Most commonly involved cell types in neuroinflammation are microglia and astrocytes. These cells are capable of clearing $A \beta$-deposits through phago-lysosomal degradation (Frackowiak et al., 1992; Wisniewski et al., 1991; Wyss-Coray et al., 2003). Briefly, phagocytosis is a specific form of endocytosis by which the cell membrane engulfs solid particles or whole microorganisms from the extracellular space. The engulfed debris is gradually transported within maturating endosomes, which eventually fuse with the lysosome for enzymatic degradation of the content. The detailed molecular mechanism underlying the endo-lysosomal pathway will be described in detail in section 1.2.4.

In microglia, e.g. this phago-lysosomal activation coincides with morphologic changes displayed by cell swelling, altered gene expression and the secretion of signaling molecules like cytokines to interact with the environment (Kettenmann et al., 2011). Moreover, there is emerging evidence that microglia can also directly interact with astrocytes and vice versa, suggesting a close link between gliosis and neuroinflammation (Liddelow et al., 2017). Throughout disease progression the blood brain barrier eventually breaks down which allows peripheral immune cells to enter the brain. This includes e.g. peripheral monocytes, neutrophils and T cells (Zenaro et al., 2017). How these cells contribute to neuroinflammation in $A D$ is not well understood yet. Detailed characteristics of microgliosis in $A D$ will be discussed in detail in section 1.2.3.

\subsubsection{Amyloid pathology}

There are two primary pathways processing APP: whereas the so-called non-amyloidogenic pathway creates no $A \beta$-species, the amyloidogenic pathway is responsible for the generation of $A \beta$ peptides (Hardy and Higgins, 1992). The most important enzymes involved in these two pathways are the alpha- $(\alpha-)$, beta- $(\beta-)$ and gamma- $\left(\gamma^{-}\right)$secretases.

\section{Non-amyloidogenic pathway}

In the non-amyloidogenic pathway, APP is proteolytically cleaved by a-secretase (a disintegrin and metalloproteinase 10, ADAM10). Thereby, the soluble ectodomain, also called sAPPa, is released into the extracellular space and C83 also known as the $\alpha$-C-terminal fragment ( $\alpha$-CTF) remains 
membrane-bound (Figure $3 \mathbf{A}$ ). As the $\alpha$-cleavage site is within the $A \beta$-sequence which is thereby disrupted, A $\beta$-synthesis is prevented. In a second step, $\alpha$-CTF is then further processed by $\gamma^{-}$ secretase cleaving at the C-terminal transmembrane domain. This results in the release of a small extracellular peptide called p3 and the cytosolic APP intracellular domain (AICD) (Hardy and Higgins, 1992; Heppner et al., 2015).

\section{Amyloidogenic pathway}

In the amyloidogenic pathway the first cleaving is achieved by $\beta$-secretase ( $\beta$-site APP cleaving enzyme, BACE1), which cuts at the $C$-terminal end of the $A \beta$-sequence and releases the soluble SAPP $\beta$ residue into the extracellular space (Figure $3 \mathbf{A}$ ). The remaining $\beta$-CTF (C-terminal fragment of APP also referred to as C99) is then further processed by the $\mathrm{Y}$-secretase, resulting in membrane bound AICD and free Aß-peptide (Heppner et al., 2015). Depending on the exact cutting position of $Y$-secretase, $A \beta$-species with $C$-terminal lengths between 37- 43 amio acids are produced (Takami et al., 2009). Moreover, there are various $\mathrm{N}$-terminal truncated forms of $\mathrm{A} \beta$-peptide. $\mathrm{N}$-terminal fulllength $A \beta_{1-42}$ is considered to be highly synaptotoxic. In humans $A \beta_{2-x}$ species are highly enriched in the brain, while in transgenic $A D$ mouse model $A \beta_{3-x}$ are prominently expressed (Kummer and Heneka, 2014). In $A D$ post mortem brains variant $A \beta_{4-42}$ was found highly abundant, too (Masters et al., 1985). Thus, there must be other enzymes involved in alternative processing pathways of APP.
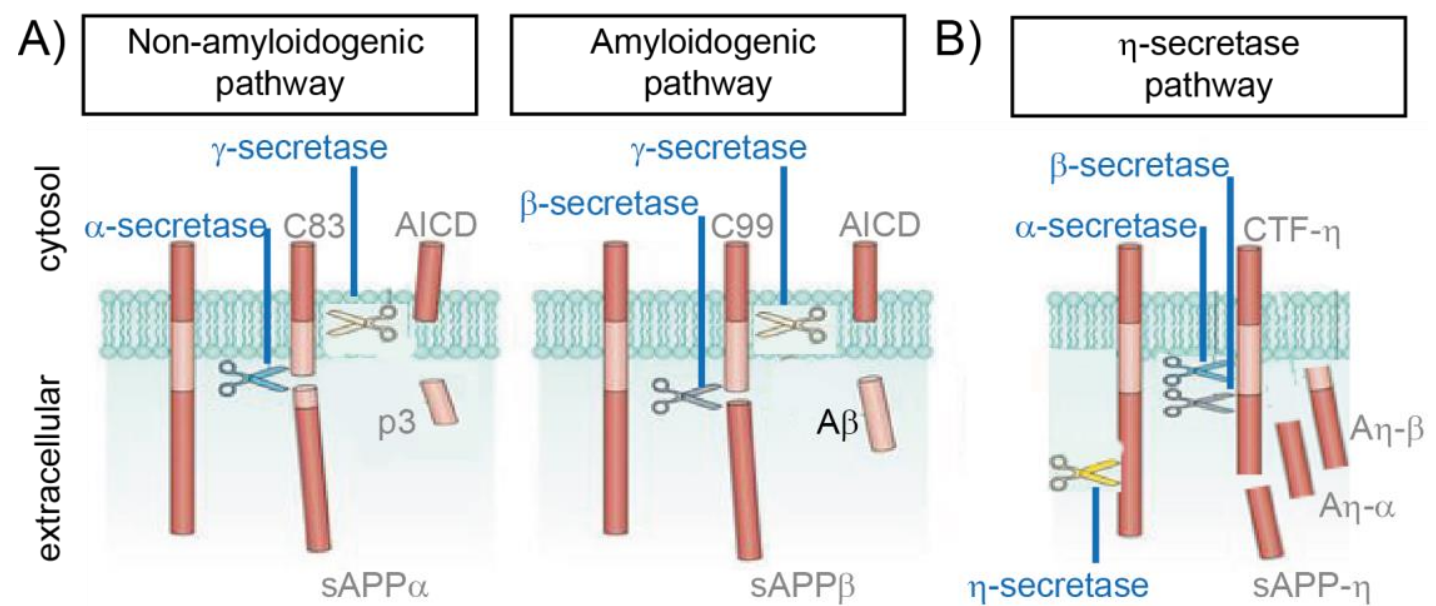

Figure 3: Schematic presentation of non-amyloidogenic, amyloidogenic and n-secretase pathway.

A) Representation of the non-amyloidogenic pathway on the left side and scheme of the amyloidogenic pathway on the right side. The A $\beta$-peptide sequence in colored in orange whereas the remaining parts of APP are colored in red. In the non-amyloidogenic processing of APP is characterize by the use of $\alpha$ - and $y$-secretases. This results in intermediate products sAPPa and C83, which is also known as a-CTF (C-terminal fragment of APP). C83 is further processed into soluble $p 3$ and membrane-bound AICD (APP intracellular domain). Through this pathway no A $\beta$ is produced. In contrast, $A \beta$ is released upon APP processing with $\beta$ - and $y$-secretases in the amyloidogenic pathway. The proteases $\alpha-, \beta$ - and $\gamma$-secretases are shown in blue. Side products which do not result in $A \beta$-monomers are labeled in grey.

B) The recently identified $\eta$-secretase pathway does not produce A $\beta$-peptides but neurotoxic An-peptides. In a first step $\eta$-secretase cleaves within the $\mathrm{N}$-terminal region of APP releasing SAPP $-\eta$. The remaining membrane-bound $\mathrm{CTF}_{-\eta}$ can be further processed either by $\alpha$ - or $\beta$-secretase resulting in $A_{n-\alpha}$ or $A_{n-\beta}$ peptides. Figure is adapted from Heppner et al., (2015). 
APP cleaving sites

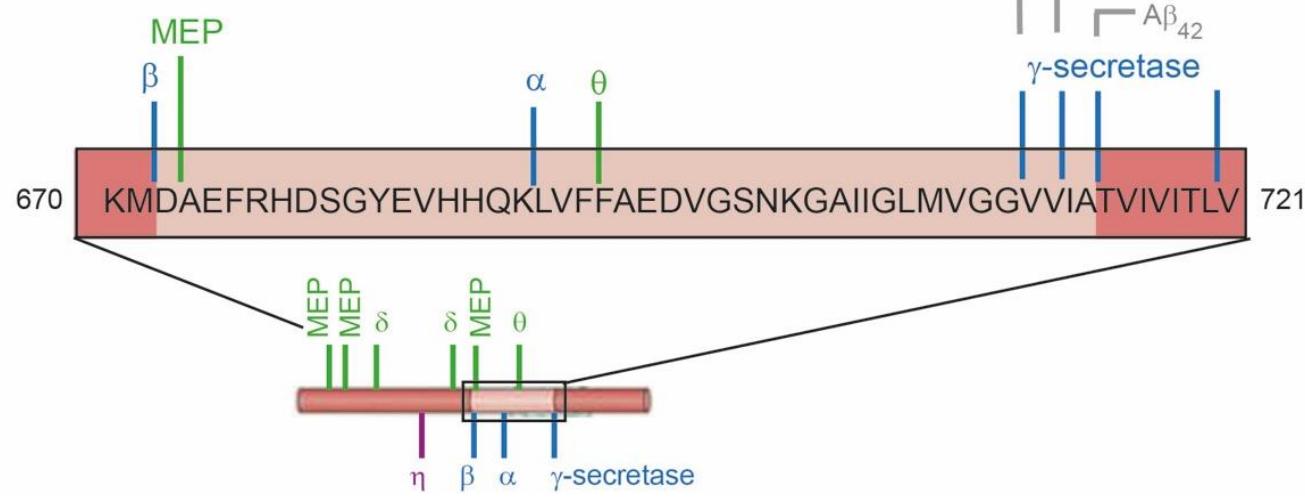

Figure 4: Cleaving sites of APP processing enzymes.

On the bottom, the full-length APP-protein is shown in red with the Aß-peptide in orange. The broad cleaving sites of multiple, prominent APP-processing enzymes are labeled. In a close-up of the amino acid (aa) sequence of $A \beta$ shown on top, the precise cleaving sites are tagged. For instance $\alpha$-, $\beta$ - and $\gamma$-secretases which cleave within the $A \beta$-peptide sequence and which are the key components of the non-amyloidogenic and amyloidogenic pathway are shown in blue. Depending on the precise cleaving site of $Y$-secretase in the amyloidogenic pathway, $A \beta$-peptides with various lengths, like 38 aa, 40 aa or 42 aa are produced (grey). Other secretases which can cut within the A $\beta$-sequence are $\theta$-secretase and MEP (Merpin) labeled in green. MEP and $\delta$-secretase can further cleave within the N-terminus of the APP. The cleaving sites of $\eta$-secretase (purple) are $\mathrm{N}$-terminal to the $\mathrm{A} \beta$-sequence.

Metalloprotease Meprin $\beta$, for example, was identified as alternative $\beta$-secretase which can generate amongst others $N$-terminal truncated $A \beta_{2-x}$-peptides (Becker-Pauly and Pietrzik, 2016; Jefferson et al., 2011). In addition to the one cleaving site inside the $A \beta$-sequence, Meprin can cut within the $\mathrm{N}$-terminus of APP. The cleaving site of theta- ( $\theta$ - also known as BACE2) secretase is within the $A \beta$-sequence, thus processing $A \beta$-peptides (Sun et al., 2006). Delta- ( $\delta-)$ secretase cuts within the N-terminus of APP (Zhang et al., 2015).

Noteworthy, a recently identified APP processing pathway, underlying eta- ( $\eta$-) secretase, also known as membrane-bound matrix metalloproteinase (MT5-MMP), does not process A $\beta$-peptides but results in neuro-toxic molecules (Willem et al., 2015) (Figure 3 B). In a first step n-secretase cleaves APP between amino acid 504 and 505 which is closer to the $\mathrm{N}$-terminus than the cleaving site of the $\beta$-secretase (Figure $3 \mathbf{B}$ ). Thereby, the soluble sAPPn fragment is released. The membrane-bound CTF $\eta$ is either degraded through the lysosomal pathway or further processed by $\alpha$ - or $\beta$-secretase resulting in soluble A $\eta-x$ peptide and membrane-bound CTFx- $\eta$ (Wang et al., 2015a; Willem et al., 2015). Interestingly, elevated levels of CTFx- $\eta$ were found in dystrophic neurites in a human APP-overexpressing AD mouse model and in human AD post mortem brains (Willem et al., 2015). Comparable to treatment with BACE1 inhibitors, An- $\alpha$ was found to reduce neuronal activity in murine hippocampal slice culture (Willem et al., 2015). KO of MT5-MMP in the $5 x F A D$ transgenic $A D$ mouse model showed attenuated $A \beta$ burden, reduced gliosis and cognitive 
improvement compared to control animals (Baranger et al., 2017). This further illustrates the capacity of non-A $\beta$-species to influence $A D$-pathology.

In terms of $A \beta$-peptides, $A \beta_{42}$ is considered the most pathogenic form due to its strong tendency to form aggregates. After $A \beta$-peptides are released into the extracellular space, they gradually start to accumulate and to build energetically more stable, higher molecular aggregates: small oligomers, fibrils and finally $A \beta$-plaques. Aggregation properties of $A \beta$-peptides can be further altered by posttranslational modifications such as oxidation, phosphorylation, glycosylation or pyroglutamylation. For instance, pyroglutamylation at $\mathrm{N}$-terminal position 3 of $A \beta$-peptides $\left(A \beta_{p E 3}\right)$ impedes $A \beta$-fibril assembly while triggering formation of $A \beta$-oligomers which are having comparable neurotoxic properties to $A \beta_{1-42}$ (Saido et al., 1995; Tekirian et al., 1999; Warner et al., 2016; Youssef et al., 2008). Additionally, $A \beta_{p E 3}$ was discovered to reverse $A \beta_{1-42}$ fibrils into oligomers in a prion-like manner, which further enhances its neurotoxic potential (Nussbaum et al., 2012; Schilling et al., 2006).

$\mathrm{A} \beta$-peptides can also accumulate intracellularly and thereby induce cell toxicity amongst others by stress induced damage of cell organelles (Cabrejo et al., 2006; D'Andrea et al., 2002; Gouras et al., 2000; LaFerla et al., 1997; Umeda et al., 2011).

\section{Amyloid cascade hypothesis}

In contrast to previous believes that the amyloidogenic pathways were exclusively linked to the diseased brain, it is now well established that both pathways are present in physiological conditions as a small amount of $A \beta$-peptides is also produced in the healthy brain (Haass et al., 1992).

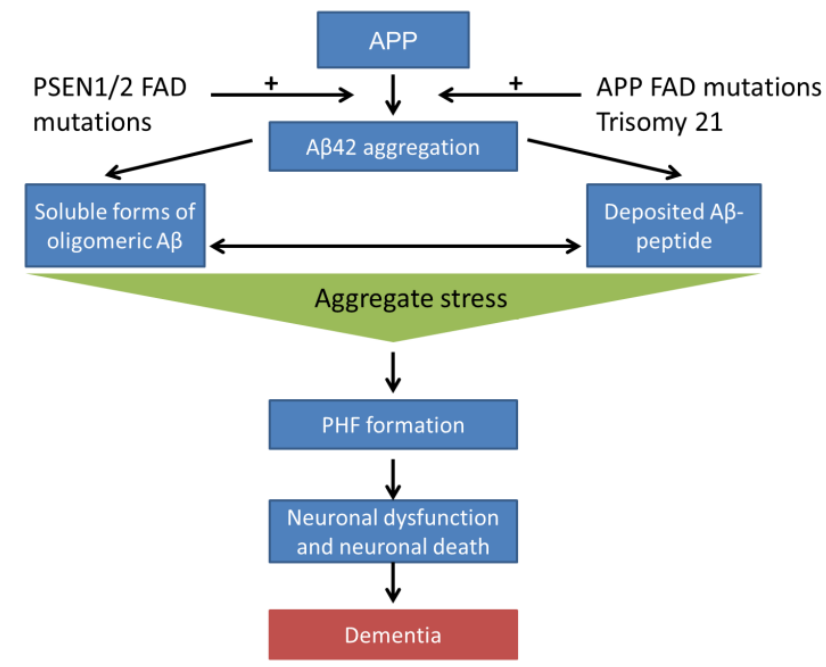

Figure 5: Amyloid cascade hypothesis.

Genetic mutations or increased copy-numbers of App result in higher concentrations of APP and thereby also in increased production of aggregation prone $A \beta_{42}$-peptides, which can aggregate to oligomers, protofibrils, fibrils and eventually into $A \beta$-plaques. These aggregation states can be modulated. All of these $A \beta$-aggregates induce cellular stress which triggers intraneuronal formation of paired helical filaments (PHFs) of pTau. This in turn contributes to neuronal dysfunction and cell death resulting in neuronal cell loss and ultimately leads to functional deficits as refleced in dementia. Figure adapted from Karran et al., (2011). 
However, it is now reckoned that there is a balance in production and clearance of $A \beta$-species under healthy, physiological conditions, which is impaired in AD (Figure 3 B). Increased concentrations of $A \beta$-peptides are believed to facilitate extracellular and intraneuronal aggregation. Therefore, the amyloid cascade hypothesis suggests that toxic $A \beta$ oligomers are causative for downstream AD pathology including neuronal pTau aggregation, contributing to synaptic dysfunction, neuronal loss and secondary neuroinflammation and gliosis. All of these events eventually lead to dementia (Figure 5). This hypothesis is supported by various mutations found in rare, genetic inherited forms of $A D$ (familial $A D$ or $F A D$ ) that enhance the production of aggregation prone $A \beta_{42}$-peptides. In addition, a higher gene dosage of $A P P$, which is found in Down syndrome due to a third copy of $A P P$, results in the deposition of $A \beta$ at a young age (Lemere et al., 1996).

\subsubsection{Risk factors for Alzheimer's disease}

Alzheimer's disease is known to be a complex and multifactorial disease that involves environmental, epigenetic and genetic factors (Huang and Mucke, 2012).

\subsubsection{Environmental factors}

$A D$ is a typical disease of the aged and thus, aging itself is the highest risk factor. Also, it is strongly linked to poor education, mental activity, physical fitness, obesity, diet and lifestyle (Lindsay et al., 2002; Medina et al., 2017). Moreover, pre-existing medical conditions, including cardio-vascular diseases, periodontitis, brain trauma, sepsis or systemic inflammation increase the risk to develop AD (Lindsay et al., 2002; Medina et al., 2017). Mental illnesses, like depression, but also hearing loss, which could further contribute to social isolation, are also well documented risk factors for the development of $A D$ (Livingston et al., 2017). Smoking and heavy alcohol consumption are associated with increased AD risk, too (Lindsay et al., 2002; Medina et al., 2017). These findings led to the conclusion that living a "healthy lifestyle" can already help to reduce the risk of AD by about 20\% (Livingston et al., 2017).

\subsubsection{Risk genes in sporadic AD}

Genome-wide association studies (GWAS) have helped to identify several genes which are associated with increased risk to develop sporadic forms of $A D$. Identified risk genes are amongst others TREM2 (Triggering receptor expressed in myeloid cells 2), DAP12 (DNAX-activating protein), ApoE, CR1 (complement receptor 1), CD33 (also known as Siglec-3, sialic acid-binding immunoglobulin-like lectin), MHC II (major histocompatibility complex class II, also known as HLADRB5-DRB1) (Hollingworth et al., 2011; Lambert et al., 2009; Lambert et al., 2013). The most prominent and thus far best characterized ones are ApoE and TREM2 which will be described in the following in more detail. 


\section{Apoprotein E (APOE)}

The highest genetic risk factor for late onset AD is Apoprotein E (ApoE) (Holtzman et al., 2000a; Holtzman et al., 2000b). ApoE is a lipid binding protein, which is composed of 299 amino acids and which has three isoforms, ApoE2, ApoE3 and ApoE4, in humans (Rebeck et al., 1993). The isoforms differ only by two amino acids at position 112 and 158, respectively: ApoE2 (Cys112, Cys158), ApoE3 (Cys112, Arg158), and ApoE4 (Arg112, Arg158) (Mahley and Rall, 2000). In particular, carriers homozygous for isoform ApoE4 are associated with an increased risk to develop $A D$ and with earlier onset of the disease (Mahley et al., 2006). In the early 1990s ApoE was found to cluster around senile $A \beta$-plaques in post mortem brain samples of $A D$ patients (Namba et al., 1991; Strittmatter et al., 1993a; Strittmatter et al., 1993b).

Under physiological conditions $A p o E$ is predominantly expressed by astrocytes, whereas $A \beta$ exposure causes increased ApoE expression by microglia (Uchihara et al., 1995). ApoE binds monomeric $A \beta$-peptides and thereby facilitate the degradation by microglial phagocytic uptake (Lee et al., 2012). ApoE4 has lower functional capacity of $A \beta$-clearance in comparison to the other two isoforms of ApoE (Castano et al., 1995; Castellano et al., 2011; Holtzman et al., 2000b; Lin et al., 2018; Strittmatter et al., 1993b). Accordingly, ApoE4 also shows slower kinetics in Aß-clearance at the blood brain barrier compared to ApoE2 and ApoE3, as demonstrated in BL6 mice, which were microinjected with $A \beta$ and the respective ApoE species (Deane et al., 2008). Studies in transgenic mice expressing human ApoE4, but not ApoE2 and ApoE3, also found indications that ApoE4 could contribute to blood brain barrier breakdown (Bell et al., 2012).

Genetic KO of ApoE in APP-overexpressing mice resulted in dramatic reduction of diffuse $A \beta$-plaques and diminished dystrophic neurites (Bales et al., 1997; Holtzman et al., 2000a). However, using a more aggressive $A D$ model with additional mutation within $y$-secretase subunit PSEN1, ApoE ablation did not reduce $A \beta$ load as strongly as in the previously described models (Ulrich et al., 2018). Nonetheless, ApoE KO resulted in altered A $\beta$-plaque distribution, decreased dystrophic neurites and in reduced microgliosis while changing transcription of inflammatory profiles in hippocampal tissue (Ulrich et al., 2018). These findings led to the conclusion that ApoE facilitates recruitment of microglia around $A \beta$-plaques and reduces $A \beta$-mediated neuronal toxicity.

Besides modulating glial cells, ApoE was found to directly stimulate the transcription of APP in vitro and in mice. ApoE4 was identified as the most potent activator of a non-canonical MAP (mitogenactivated protein) kinase pathway which enhances transcription of APP and thereby increases the concentration of $A \beta$, too (Huang et al., 2017).

\section{Triggering receptor expressed in myeloid cells 2 (TREM2)}

Exome, genome and transcriptome analysis of $A D$ patients and controls identified the missense mutation $\mathrm{R} 47 \mathrm{H}$ in triggering receptor expressed in myeloid cells 2 (TREM2) to be highly associated with development of AD disease (Guerreiro et al., 2013; Kleinberger et al., 2014). TREM2 is a cell 
surface marker expressed by myeloid cells, where it regulates phagocytosis by activating DAP12 which is an AD risk gene as well (Kleinberger et al., 2014; Takahashi et al., 2007).

AD mouse models deficient in Trem2 showed the importance of the underlying signaling pathway to clear $A \beta$-peptides: microglia with Trem2 $\mathrm{KO}$ could not migrate towards $A \beta$-plaques and became apoptotic. Moreover, these Trem2 KO mice showed less dense and more diffuse A $\beta$-plaques as well as higher damage of neurites (Wang et al., 2015b; Wang et al., 2016). Overexpression of Trem2 in 5xFAD mice resulted in upregulation of phagocytosis genes in $A \beta$-plaques associated microglia and downregulation of immune cell activation genes. Furthermore, these animals showed less dystrophic neurites and improved memory (Lee et al., 2018).

Current data identified ApoE to be a ligand of TREM2 (Atagi et al., 2015; Yeh et al., 2016). Transcriptome and proteome analyses of APP/PS1 mice identified a Trem2-ApoE pathway, which affects microglia behavior; as microglia clear dystrophic neurites by Trem2-mediated phagocytosis, an ApoE dependent downstream pathway is activated. This signaling pathway subsequently suppresses the homeostatic microglia phenotype and shifts the cells into a disease associated state (Krasemann et al., 2017). This microglial ApoE pathway was also found in mouse models of amyotrophic lateral sclerosis (ALS) and multiple sclerosis (Krasemann et al., 2017).

In addition to the link of these two $A D$ risk genes, transcriptome analysis of human blood-derived monocytes of $A D$ patients revealed a correlation between the expression of TREM2 and $A D$ risk gene CD33 (Chan et al., 2015).

Notably, mutations within the homologue TREM1 were also reported to increase the risk of $A D$ (Replogle et al., 2015). However, this receptor is triggering different downstream signaling pathways and is more involved in the activation of pro-inflammatory cytokine secretion. In comparison to Trem2 Trem1 shows opposing gene regulation upon LPS activation (Owens et al., 2017). Moreover, Trem1 susceptibility allele rs6910730G correlates with decreased receptor expression and lower Trem1 / Trem2 RNA levels (Chan et al., 2015).

Even though TREM2 is the best characterized microglia-expressed $A D$ risk gene, several screenings of microglia from rodent $A D$ models and human $A D$ post mortem tissue, as well as whole tissue GWAS analyses identified various genes to be differentially expressed by myeloid cells, including microglia, in context of AD (Gosselin et al., 2017; Hollingworth et al., 2011; Lambert et al., 2009; Lambert et al., 2013). This includes late-onset AD risk genes such as DAP12, CD33 or CR1. In conclusion, there is a close link between sporadic $A D$ development and various $A D$ risk genes expressed by microglia which are associated with various microglia functions.

\subsubsection{Familial AD}

As mentioned, most AD cases are sporadic. However, in few cases genetic mutations within APP or within Presinelin1 and 2 (PSEN1, PSEN2) are responsible for the development of AD. These familial $A D(F A D)$ cases are inherited autosomal dominantly (De Jonghe et al., 2001; De Strooper 


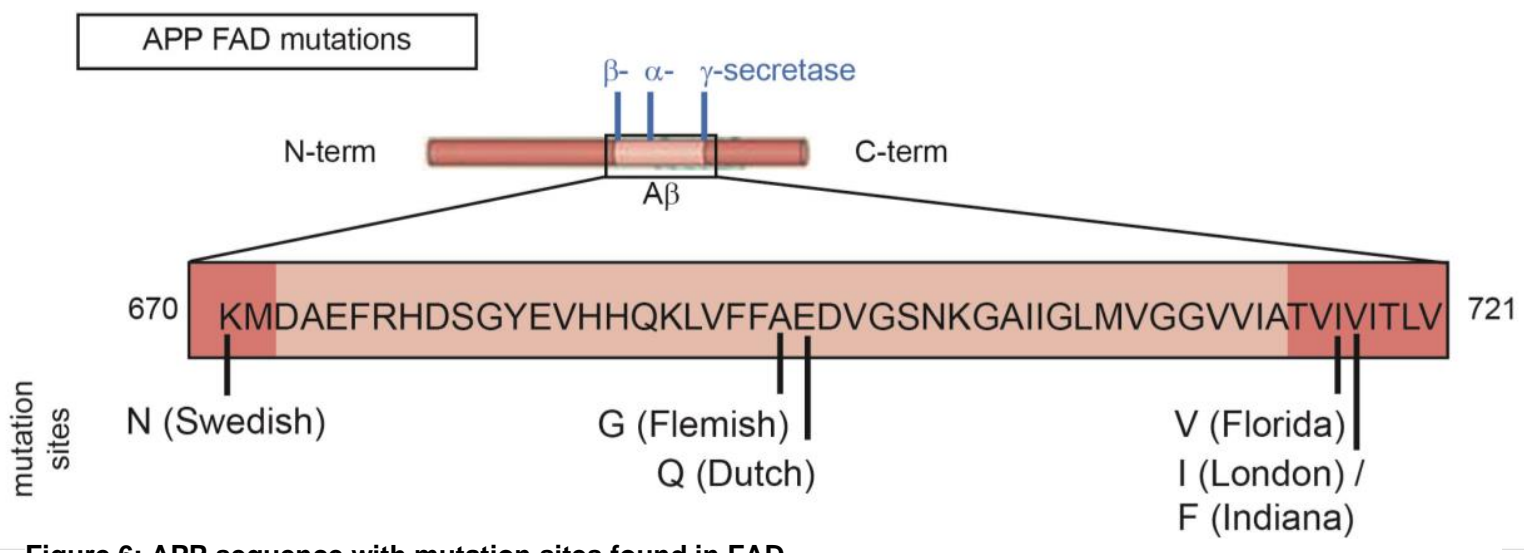

Figure 6: APP sequence with mutation sites found in FAD.

The extracellular N-terminus of APP in the membrane-bound and cytosolic oriented C-terminus of APP are labeled in red. The $A \beta$-sequence is shown in orange. Cleaving sites for $\alpha-, \beta$ - and $y$-secretases (blue) are indicated within the $A \beta$ region. The close-up on the bottom shows the aa sequence of $A \beta$. A selection of known point mutations within APP of FAD cases are shown: "Swedish": K595N/M596L; "Flemish”: A692G; "Dutch”: E693Q; "Florida”: 1716V; "London": V717I and "Indiana": V717F. Mutation sites according to Eckman et al., (1997; Hyttinen et al., 2013), Goate et al., (1991), Hendriks et al. (1992), Levy et al., (1990), Mullan et al., (1992) and Murrell et al., (1991).

et al., 2012; Goate et al., 1991). Mutations within these genes have direct or indirect consequences on the processing of APP and thereby on increased production of $A \beta$-species.

\section{Mutations in Amyloid precursor protein (APP)}

Mutations within the APP facilitate the proteolytic processing of A $\beta$-peptide and thereby increase the amount of $A \beta$-peptides, accumulating to $A \beta$-plaques in the extracellular space. Some of the best characterized mutations within APP include the point mutations A692G (missense, "Flemish") (Hendriks et al., 1992), K595N/M596L (point, "Swedish"), (Mullan et al., 1992), E693Q (missense, "Dutch" (Levy et al., 1990), V717I (missense, "London") (Goate et al., 1991), V717F (“Indiana") (Murrell et al., 1991) and I716V (missense, "Florida") (Eckman et al., 1997) (Figure 6). As of today there are more than 50 pathogenic mutations found within APP (Weggen and Beher, 2012). Depending on the mutation site, APP processing is altered. For instance the "Swedish" mutation results in higher affinity binding of the BACE1 secretase to APP and thereby increased processing of $A \beta$ (Cai et al., 2001; Das et al., 2015).

\section{Mutations in Presenilin 1 and 2 (PSEN1 +2)}

Mutations within the PSEN1 and PSEN2 genes are found in inherited FAD (Levy-Lahad et al., 1995; Rogaev et al., 1995). PSEN1 is located on chromosome 14, whereas PSEN2 lies on chromosome 1. Both genes encode for a subunit of the $y$-secretase. Numerous mutations are reported in PSEN1 (up to today more than 180), mutations within PSEN2 are less frequent (Weggen and Beher, 2012). Mutations within PSEN1 and PSEN2 increase $A \beta_{42} / A \beta_{40}$ ratios, associated with increased risk of AD (De Jonghe et al., 1999; Levy-Lahad et al., 1995; Rogaev et al., 1995). 


\subsubsection{Transgenic AD mouse models}

To investigate $A D$ in vivo, many mouse models were generated based on genetic mutations found in FAD cases as illustrated for APP in Figure 6. Due to the fact that rodents do not develop AD, these mutations were introduced by transgenic overexpression of the mutated human sequences of APP, PSEN1 or PSEN2.

One of the first generated AD mouse lines was the PDAPP line, which overexpresses human APP bearing the Indiana mutation (Games et al., 1995). Various other mouse lines with one or more FAD mutations in APP were generated throughout the years, e.g. Tg2576 (Hsiao et al., 1996) and TgCRND8 (Chishti et al., 2001). These mouse lines mimic the A $\beta$-pathology, dystrophic neurite pathology and neuronal loss well.

However, to achieve an earlier onset of the disease by having increased $A \beta$-levels, double transgenic mouse lines, which also overexpress FAD forms of PSEN1 and PSEN2 were generated. The most prominent ones are APPPS1-21 (Radde et al., 2006), APP/PS1 (APP/PS1 $\triangle$ E9) (Borchelt et al., 1997) and 5XFAD (Oakley et al., 2006). APPPS1-21 mice overexpress APP harboring the Swedish mutation and PSEN1 containing the L166P FAD mutation. By 6 weeks of age these mice reveal $A \beta$-deposits in the cortex, astro- and microgliosis. However, increased neuronal loss is only detected in 17-month old mice (Rupp et al., 2011). APP/PS1 mice overexpress the APP containing the Swedish mutation and PSEN1 with deleted exon 9. Both transgenes are under transcriptional control of the prion promoter. APP/PS1 mice only show AB-deposits at 6 months of age (Reiserer et al., 2007).

The mouse model of choice for this project was the 5xFAD (five times familial Alzheimer's disease) mouse line. This mouse model is mimicking several aspects of $A D$ pathology with a rapid development of $A \beta$-pathology due to five point mutations found in FAD cases: three point mutations are located within the APP gene, known as Florida (1716V), London (V717I) and Swedish (K670N) mutations, and additional two mutations within the PSEN1 gene, more precisely the point mutations M146L and L286. To achieve neuronal overexpression of those genes, they were introduced under transcriptional control of the neuron-specific Thy1-promoter (Oakley et al., 2006). By 2 months of age, 5xFAD mice already display $A \beta$-plaques in the subiculum and partially other parts of the hippocampus and within cortex layer $\mathrm{V}$. By 9 months of age the animals show A $\beta$-plaques in most brain regions. By this time, also neuronal, synaptic and dendritic spine loss as well as astro- and microgliosis are observed in these mice. The neuropathology is paralleled by impaired contextual and spatial memory which can be detected as early as 5 months of age. By 6 months of age, longterm potentiation and depression (LTP/LDP), two events crucial for learning, are affected in these mice (Kimura and Ohno, 2009). Therefore, the 5xFAD represents a good model, which recapitulates amyloid pathology and neuronal loss to investigate early and fast $A D$ progression due to $A \beta$-overexpression in vivo. 


\subsection{Microglia}

Microglia are the innate immune cells of the brain that belong to the myeloid cell lineage. They were first described in 1932 by Pío del Río Hortega using silver staining (Ramon y Cajal Agüeras, 2016). The microglia cell population comprises of $5-12 \%$ to the whole cell population of the brain (Spittau, 2017). As immune cells, their main function is to remove debris and pathogens from the CNS.

This is of great importance for neuronal development during embryonic development but also during neurological and neurodegenerative diseases (Lenz and Nelson, 2018; Spittau, 2017). Furthermore, as previously introduced, recent studies identified mutations within myeloid genes to be present in late-onset $A D$ cases (see section 1.1.3.2). Therefore, the role of microglia in $A D$ development and progression seems to have more aspects than we are aware of today.

\subsubsection{Microglia development and maintenance}

Whereas neurons, astrocytes and oligodendrocytes originate from a common progenitor developed in the neural tube, microglia arise from the yolk sac (Kessaris et al., 2008; Kierdorf et al., 2013). Their development is similar to other myeloid cells, which share common erythro-myeloid progenitors (EMPs) (Figure 7). Differentiation of EMPs is mostly driven by PU.1 (also known as Sfpi) which is the main transcription factor of myeloid cells. At E9.5 $\mathrm{CX}_{3} \mathrm{CR} 1+(\mathrm{CX} 3 \mathrm{C}$ chemokine receptor 1 also known as the fractalkine receptor positive) progenitor cells originating from the yolk sac migrate into their future domestic location (Kierdorf et al., 2013). Within these anatomical locations the resident precursors further differentiate into tissue-specific macrophages at around E10.25, including future microglia in the brain (Mass et al., 2016). To achieve differentiation into those cellular subtypes, different genes are expressed after the cells have arrived in their domestic anatomical location. In case of microglia, the $\mathrm{CX}_{3} \mathrm{CR} 1+$ precursors migrate to the future brain parenchyma with the help of matrix metalloproteinases. At this stage the microglia progenitors feature an amoeboid morphology. By embryonic day E13.5 in mice microglia precursors can be detected in the fourth ventricle (Arnò et al., 2014). Settling in the neuroectoderm, microglia progenitors start to locally proliferate and thereby to expand the cell colony to a stable population of ramified microglia in the murine brain at P28 (Bennett et al., 2016). By the end of the second postnatal phase, between P21 and two months, microglia feature gene expressions and morphological phenotypes of adult microglia (Bennett et al., 2016; Butovsky et al., 2014; Matcovitch-Natan et al., 2016). Interestingly, microglia development in male and female mice shows some fundamental differences: whereas male mice have higher numbers and amoeboid shaped microglia during embryonic and neonatal development, females feature fewer but ramified cells (Lenz et al., 2013; Schwarz et al., 2012). Within the first month after birth these disparities are mitigated as the numbers in females increase while the morphology in males changes towards 


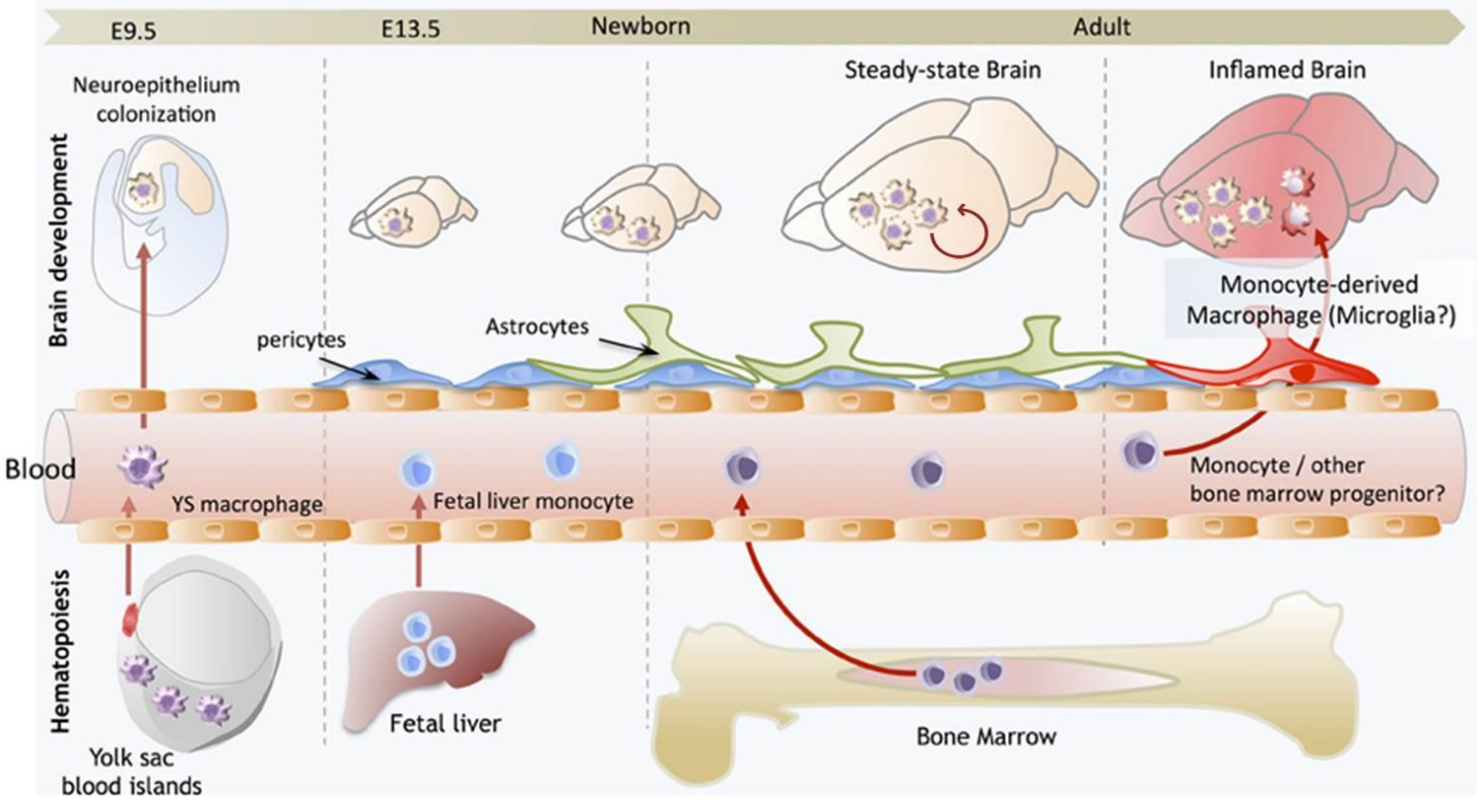

Figure 7: Microglia development and maintenance in the rodent brain.

During embryonic development $\mathrm{CX}_{3} \mathrm{CR} 1+$ precursors from the yolk sac micgrate to the neuroepithelium which is the origin of the brain. By embronic day 13.5 (E13.5) these precursors begin to differentiate and to migrate towards the neuroectoderm. Local cell proliferation starts and peaks after birth. Already at E13.5 the blood brain barrier starts to develop by recruiting pericytes towards blood vessels. Hence, fetal liver-derived monocytes are excluded from the microglia pool, but give rise to CNS macrophages such as perivascular macrophages. By the time of birth the blood brain barrier is fully closed through pericytes and astrocytes. Consequently, bone-marrow derived blood monocytes cannot enter the brain under physiological conditions, allowing microglia to only maintain themselves through cell division and apoptosis. However, in case of brain inflammation the blood brain barrier opens allowing blood-derived monocytes to enter the brain. Figures is modified after Ginhoux et al., (2013).

ramified cells (Schwarz et al., 2012). These features are associated with reduced phagocytic activity of male microglia (Lenz and Nelson, 2018; Lenz et al., 2013; Yanguas-Casas et al., 2018).

As the differentiation of microglia progenitors happens beyond the blood brain barrier, and thereby in interaction with the neuronal environment, these cells display gene expression profiles that highly differ from those of other tissue macrophages (Gautier et al., 2012). However, they share a lot of markers with other myeloid cells due to their common origins (Mass et al., 2016).

Under physiological conditions, reflected by intact blood brain barrier, microglia maintain themselves throughout adulthood by cell division and apoptosis. Hence, they are basically independent of the hematopoetic stem cell pool (Bruttger et al., 2015; Hashimoto et al., 2013; Tay et al., 2017). For instance, a parabiosis study using a mouse with depleted bone marrow-derived phagocytes conjoint to a mouse with GFP-labeled bone marrow-derived monocytes detected only small degree of infiltrating GFP+ cells in the brain of the recipient mouse (Wang et al., 2016). In addition, data obtained from human post mortem samples verified slow, but steady self-renewal of microglia with overall stable cell numbers throughout adulthood (Askew et al., 2017; Réu et al., 
2017). Therefore, microglia maintenance is mostly autarkic which is in contrast to many other tissue macrophages, which undergo physiological turnover by cell differentiation from hematopoietic precursors (Ginhoux et al., 2010).

However, radiation experiments, which damaged the blood brain barrier, showed infiltration of circulating macrophages to the brain and temporary aid of macrophages in neuroinflammation (Ajami et al., 2007; Mildner et al., 2007). Since leakiness of the blood brain barrier is observed in the chronically inflamed brain, e.g. as in progressed neurodegenerative diseases, infiltrating peripheral immune cells are likely to also play a role in $A D$.

Even though peripheral myeloid cells do not contribute to the microglia pool under physiological conditions, it needs to be mentioned that myeloid cells within the dural lymapthic vessels and along the blood-brain and blood-CSF barrier might also be involved in immune-regulation of the brain (Lapenna et al., 2018; Prinz et al., 2011). This includes perivascular macrophages, meningeal macrophages, choroid plexus macrophages and monocytes from the blood stream. These cells are considered as a potential mechanism for communication of microglia with the peripheral immune system (Lapenna et al., 2018; Prinz et al., 2011).

\subsubsection{Microglia function throughout aging}

The main roles of microglia include developmental aid, repair and damage response and maintenance of the neural environment (Lenz and Nelson, 2018). During embryogenesis and within the first postnatal days, microglia are essential for proper CNS development and homeostasis. For instance, early genetic and pharmacological ablation of microglia by targeting CSF1R (colony stimulating factor 1 receptor) resulted in disturbed outgrowth of dopaminergic axons in the forebrain of mice. Additionally, some neocortical interneurons were falsely positioned showing the importance of microglia for axonal guidance during development (Squarzoni et al., 2014). Microglia also support myelination of axons during early postnatal development (Lenz and Nelson, 2018; Mosser et al., 2017). In early development, microglia are crucial for synaptic patterning through phagocytosis of cellular debris (Boulanger, 2009). Depending on neuronal activity, microglia engulf and digest presynaptic inputs (Paolicelli et al., 2011).

In the adult organism the most important and best studied task of microglia is their aid in responses to injuries and pathogens. Microglia use their motile processes to constantly screen their surrounding for pathogenic cues (Davalos et al., 2005; Nimmerjahn et al., 2005). Microglia can be activated by direct pathogen binding or by stimulation through cytokines and chemokines. Both forms of activation can be mediated by various surface molecules commonly expressed along the myeloid lineages. This includes $\mathrm{MHCl}$ and $\mathrm{MHCll}$, Toll-like receptors (TLRs), including TLR2, TRL3, TLR4 and TLR9, and several cluster of differentiation (CD) proteins, such as CD11b, CD11c and CD18 (Kettenmann et al., 2011; Trudler et al., 2010). Several of these molecules are upregulated 
during aging, such as MHCII, TLR2, 4 and 7, CD68 and CD86 (Griffin et al., 2006; Letiembre et al., 2007; Ogura et al., 1994; VanGuilder et al., 2011; Wong et al., 2005). This emphasizes the higher propensity of aged microglia for acquiring an activated phenotype. Upon activation, downstream signaling pathways induce transcriptional changes causing morphological transformation, altered secretion of cytokines and chemokines, and enhanced phago-lysosomal activity.

\section{Morphology}

In terms of morphological changes, resting microglia are characterized by a small soma with long and thin processes, constantly surveying their immediate surroundings for pathogens or injuries (Davalos et al., 2005; Nimmerjahn et al., 2005). Once activated microglia become amoeboid with a roundish shape due to increased soma and swollen and shortened processes (Nimmerjahn et al., 2005).

In aging the basal morphological state of microglia is shifted into a rather activated state manifesting itself in amoeboid-like shape (Spittau, 2017). Moreover, there are reports observing dystrophic states of microglia during aging (Streit et al., 2004). However, it remains uncertain, whether these changes in morphology represent degeneration of microglia (Tischer et al., 2016).

\section{Cytokines and chemokines}

Also, the secretory profile of microglia is changed upon activation and depends on the type of stimulus as well as its length and its duration (Jung et al., 2005; Kettenmann et al., 2011; Yao et al., 2013). In comparison to young microglia, cells from aged rodents revealed higher basal amounts of pro-inflammatory cytokines such as TNF- $\alpha$, IL-6 and NO (Kettenmann et al., 2011). For instance, in vitro experiments with young and aged murine microglia showed increased levels of TNF- $\alpha$ and increased NO (nitric oxide) in the cell culture medium of the aged cells (Lai et al., 2013). In addition, ex vivo experiments with microglia from aged mice revealed higher levels of IL- 6 and TNF- $\alpha$ compared to microglia from young mice (Njie et al., 2012).

By secretion of cytokines and chemokines microglia can interact with their microenvironment: this involves interaction with other brain resident cells like astrocytes and neurons but also with other microglia and potentially peripheral immune cells (Osman et al., 2017; Vay et al., 2018).

The secretion of distinct chemokines, such as CCL2, builds a chemotactic gradient allowing myeloid cells to become motile and migrate towards a lesion site or a herd of infection (El Khoury et al., 2007). This process is called chemotaxis. Thereby more cells from the neighboring environment can be recruited to the lesion site. However, during physiological aging the ability of microglia for chemotaxis and process motility are strongly diminished (Moraga et al., 2015). Hence, laser induced brain injury resulted in decreased migration of lba1+ (ionized calcium-binding adapter molecule 1 positive) microglia towards the lesion site in aged animals compared to you counterparts (Damani et al., 2011). Nonetheless, CCL2 is upregulated in the brain during aging. Since the blood 
brain barrier becomes leaky throughout aging, this also enables CCR2-expressing peripheral derived monocytes to enter the brain and thereby to compensate for aged microglia (Malm et al., 2005; Roberts et al., 2012; Simard et al., 2006; Stalder et al., 2005; Varvel et al., 2016). This could also explain dissenting findings about changes in overall microglia numbers during aging: whereas studies in rhesus monkey described increasing numbers of microglia during aging, findings in rats did not show alterations in Iba1+ cell counts (Peters et al., 1991; VanGuilder et al., 2011). Since peripheral macrophages also express lba1+, this marker does not allow to distinguish them from brain resident microglia.

\section{Phago-lysosomal activity}

Microglial phagocytic uptake can be mediated by several receptors and modulated by various molecules. The complement system, for instance, improves (complements) the capacity of phagocytes, such as microglia, to clear debris and to promote inflammation. Due to a triggering stimulus, systemic proteases can cleave inactive-pro-proteins of complement factors such as $\mathrm{C} 1$, C3 and C4 and thereby activate them.

Knockout studies of several complement factors such as C1q and C3 or of complement receptor CR3 (also known as macrophage-1 antigen (Mac-1)) elucidated the importance of this signaling pathway on the development of synapses (Schafer et al., 2012; Stevens et al., 2007). For instance, C1q was reported to target synapses to mark them for phagocytic removal by microglia (Stevens et al., 2007). On microglia, CR3 and CR4 (complement receptor 4), which are heterodimers of CD18 and CD11b or CD11c, respectively, are expressed (Kettenmann et al., 2011).

During aging, transcription and protein levels of molecules involved in phagocytosis including complement factors like C1q, C3, C4 and C5, are highly upregulated in microglia of different organisms (Flowers et al., 2017; Peters et al., 1991; Reichwald et al., 2009; Ritzel et al., 2015). Alongside activation-associated markers like MHCII, CD40 and CD80 are found upregulated in microglia of aged mice compared to young counterparts (Lynch et al., 2010). Also molecules involved in phagocytosis downstream mechanism like lysosomal degradation are highly upregulated in aging. One example for this is CD68 (also known as lysosome-associated membrane protein LAMP4), which is a molecule shuttling from the plasma membrane to the lysosome and therefore is considered a marker for endocytic-lysosomal activation and microglia activity (Wong et al., 2009). Additionally, lysosome-associated membrane protein 1 and 2 (LAMP1 and LAMP2), which are expressed on lysosomes, are upregulated in microglia of aged mice (Flowers et al., 2017). These markers are considered indicators for activated microglia (Henry et al., 2009). Whereas phago-lysosomal markers are upregulated on transcriptional and translational levels, the efficiency of phagocytosis and downstream protein degradation by the endosomallysosomal cascade are strongly diminished in microglia in aging (Peters et al., 1991; Ritzel et al., 2015; Safaiyan et al., 2016). 


\section{“Inflamm-aging"}

All these findings highlight the overall shift of aged microglia towards a basal inflammatory state which is also referred to as primed state. While young microglia can return into a homeostatic state after activation, primed microglia are stuck in their inflamed activity, resulting in chronic inflammation. To better describe this microglial state of chronic inflammation in aging, the term "inflamm-aging" was introduced (Franceschi et al., 2000). "Inflamm-aging" also emphasizes the altered characteristics of microglia in aging, thus it draws attention to these cells in age-associated diseases like Alzheimer's disease.

\subsubsection{Microglia in Alzheimer's disease}

One of the hallmarks of $A D$ is microgliosis. More precisely, activated microglia cluster around $A \beta$ plaques where they clear $A \beta$-peptides by phagocytic uptake and endo-lysosomal degradation (Frackowiak et al., 1992; Wisniewski et al., 1991). Moreover, by fencing A $\beta$-plaques they help to compress $A \beta$-peptides and thereby to limit toxicity and further $A \beta$-plaque growth (Baik et al., 2016). However, this phago-lysosomal activation contributes to chronic inflammation as revealed by hyperactivation on electrophysiological, secretory, transcriptional and translational levels in $A D$ mouse models which eventually results in neuronal, synaptic and dendritic spine loss (Knobloch and Mansuy, 2008; Plescher et al., 2018; Yin et al., 2017). Additionally, a tremendous amount of high risk genes for $A D$ was found to be expressed by microglia including Trem2, ApoE4, CD33 or CR3 (Hollingworth et al., 2011; Lambert et al., 2009; Lambert et al., 2013). Thus, the role of microglia in $A D$ is ambivalent, and will be described in more detail.

\section{Phagocytosis}

As main phagocytes of the brain, microglia in close proximity to $A \beta$-plaques are highly active in clearing $A \beta$-peptides. Longitudinal transcriptome analysis of $5 x F A D$ mice verified that throughout disease progression markers for microglia activation, phagocytosis and lysosome biogenesis were upregulated (Landel et al., 2014). Transcriptome analysis of cortical tissue of transgenic mice overexpressing AD risk gene ApoE4 verified significant enrichment of genes of the endo-lysosomal pathway (Nuriel et al., 2017). Interestingly, many of these genes are under control of transcription factors important for embryonic development, including PU.1 (Gosselin et al., 2017; Rustenhoven et al., 2018).

As presented before, the complement system facilitates the phagocytic uptake by innate immune cells. For instance, single-cell RNA sequencing identified amongst others C3 and C4 to be highly upregulated in microglia of the AD-like CK-p25 mouse model (Mathys et al., 2017). Investigation of APP mice showed also correlation between fibrillary $A B$ and $C 3$ and $C 4$ immuno-reactivity, respectively. C4 was predominantly detected around $A \beta$-plaques (Zhou et al., 2008). However, no effect on the total $A \beta$-plaque load was found. Blocking $C 3$, which is predominantly secreted from 
reactive astrocytes, resulted in increased $A \beta$-burden with no impact on neuronal loss (Maier et al., 2008; Shi et al., 2017; Wyss-Coray et al., 2002). Surprisingly, genetic KO of the microglial C3a receptor $(\mathrm{C} 3 \mathrm{aR})$ in an APP transgenic $A D$ mouse model resulted in decreased $A \beta$-burden achieved by more effective microglial $A \beta$ degradation (Czirr et al., 2017).

The genetic knockout of $\mathrm{Cq} 1$ in an APP transgenic mouse model resulted in fewer phagocytic microglia and less synaptic and neuronal loss (Hong et al., 2016). CR1 (complement receptor 1), the receptor of $\mathrm{C} 1 \mathrm{q}$, which was identified as risk gene for sporadic $A D$, was reported to bind to $A \beta$ and thereby to induce phagocytosis by activating the classical complement cascade (Brouwers et al., 2012; Jiang et al., 1994; Lambert et al., 2009).

Even though microglial phagocytosis is increased in $A D, A \beta$-burden is not reduced throughout disease progression. This gave rise to the idea that downstream endo-lysosomal degradation of $A \beta$ is dysfunctional (Hickman et al., 2008). Indeed, $A \beta$ engulfment by microglia in middle-aged 5xFAD mice resulted in re-distribution of $A \beta$-fibrils into the extracellular space, but not in proper protein degradation of A $\beta$-plaques (Baik et al., 2016; Njie et al., 2012). Since AD is a typical disease of old age, one possible explanation for impaired functional clearance of $A \beta$ could be microglial priming. Microglia from aged, non-transgenic animals were not able to phagocytic clear $A \beta$ in ex vivo organotypic slice cultures of aged APP/PS1-mouse. In contrary, microglia from young, nontransgenic mice could restore the phagocytic clearance of $A \beta$ in this model (Daria et al., 2017). Boosting microglia proliferation by treatment with macrophage colony-stimulating factor (M-CSF) resulted in efficient clearance of $A \beta$ by primary mouse microglia in vitro (Mitrasinovic et al., 2003). However, degradation of bacterial particles is not impaired in primary mouse microglia, while degradation of $A \beta$ is inefficient and results in release of undegraded $A \beta$ into the extracellular space (Chung et al., 1999).

Hence, microglia priming due to aging alone cannot explain their detrimental features on $A D$ pathology. A study found altered innate immune gene expression in microglia of 10 week old 5xFAD mice, already before the first $A \beta$-plaques could be detected (Boza-Serrano et al., 2018). Even more surprising are current data using a single somatic mutation (V600E) within protooncogene BRAF introduced to yolk-sac erythro-myeloid progenitors. BRAF is a kinase in the RASMEK-ERK signaling pathway. This early mutation within the myeloid cell lineage resulted in the lateonset development of neurodegeneration and therefore further supports the idea of microglia as contributors on AD development (Mass et al., 2017). 


\section{Activation state}

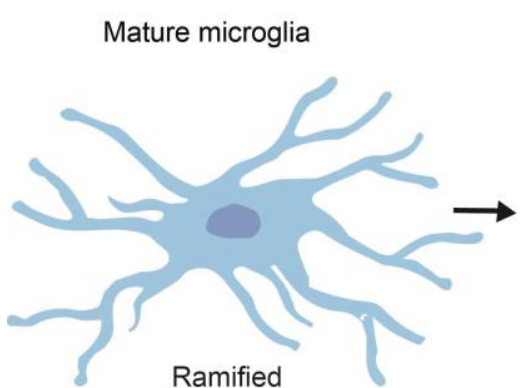

Ramified

Physiological functions:

- synaptic pruning

- shaping of axonal projections

- neuronal support

- learning-associated synapse

formation

- immune response

- injury aid
Aged microglia

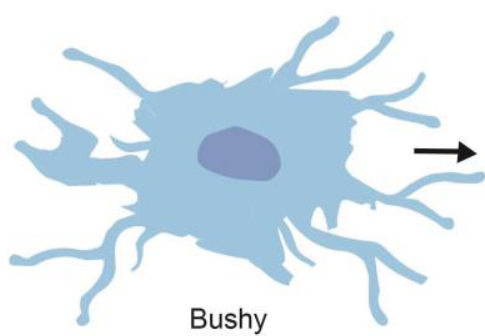

"Inflamm-aging":

- reduced phagocytosis

- reduced process mobility, chemotaxis

and migration

- increased activation

- loss of neuroprotective potential

- systemic inflammation

- less proliferative

- dystrophic
Microglia in AD

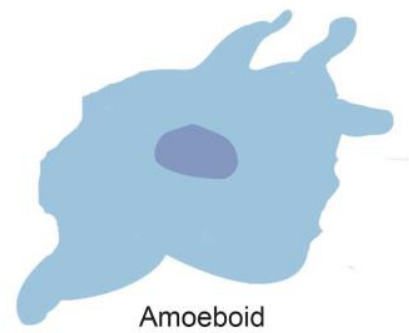

Chronic inflammation:

- increased but ineffective clearance of $A \beta$

- secretory and electrophysiological hyperactivity in proximity to $A \beta$ plaques

- neurotoxic cytokine release

Figure 8: Microglia activation throughout aging and in AD.

Left: Resting, neonatal and adult microglia are ramified, screening their environment for pathogens and injuries. They are involved in various physiological features including synaptic pruning, myelin removal, neuronal support and immune response. Middle: Throughout aging microglia shift into an activated state as seen by a rather roundish shape while they become functionally less efficient. These age-associated changes are also referred to as "inflamm-aging". Right: In $A D$ microglia are highly activated as seen by their amoeboid shape. Also they reveal less efficient endo-lysosomal activity, and secretory and electrophysiological alterations compared to cells in age-matched controls. Figures are adapted and modified after Spittau (2017) and Mosher and Wyss-Coray (2014).

\section{Disease associated microglia (DAM)}

These opposing features of microglia on AD progression could be explained by the identification of broadly heterogenic microglia subpopulations achieved by analyses of differential gene expression. Single cell RNA sequencing in 5xFAD mice and transcriptional profiling have identified three subsets of microglia (Keren-Shaul et al., 2017). One subset was called disease associated microglia (DAM). These DAM are phago-lysosomal active cells in close proximity to A $\beta$-plaques (plaque-associated microglia; PAM). DAM seem to be a subset of PAM, which increases throughout disease progression (Keren-Shaul et al., 2017). Nonetheless, it is not clear yet, which proportion of PAM corresponds to functional DAM. The DAM subpopulation was also identified in other APP overexpressing AD mouse models and in the tauopathy models Tau P301L and Tau P301S (Friedman et al., 2018; Ofengeim et al., 2017). The analyses of MHCIl-positive PAM from 5xFAD and APP23 mice identified upregulation of genes involved in immune response and phagocytosis, which were identified in DAM (Yin et al., 2017). These markers, including ApoE, AXL and Trem2, were also found in human post mortem PAM by immunohistochemistry (Yin et al., 2017).

A current study used a different mouse model to mimic rapid and aggressive neurodegeneration without amyloid pathology (CK-p25). Single cell RNA sequencing of microglia from the 
hippocampus identified 8 subpopulations of microglia, which contained 2 population of DAM (Mathys et al., 2017). These were identified as early-response and late-response microglia. The latter population shares upregulation of more than 270 genes with DAM from AD mice (Keren-Shaul et al., 2017; Mathys et al., 2017). The identification of DAM and therefore the existence of heterogenic microglial subpopulations could explain the contradictory findings of microglial impact on the progression of AD (e.g. Baik et al., 2016; Condello et al., 2015).

\section{DAM formation}

What drives microglia into transitioning towards DAM and thereby promotes the progression of $A D$ is not well understood, yet. So far, it has been described that DAM differentiation is firstly activated in a TREM2-independent manner, downregulating proteins of homeostatic microglia. This includes e.g. $\mathrm{CX}_{3} \mathrm{CR} 1$, P2ry12 and Tmem119 (Keren-Shaul et al., 2017). Then, a TREM2-dependent program is activated in these DAM inducing the upregulation of phagocytic and lysosomal markers, as well as lipid metabolites such as Lpl (lipoprotein lipase), Cst7 (cystatin F or leukocystatin), and AxI (axl receptor tyrosine kinase) (Keren-Shaul et al., 2017). These findings on transcriptional level were further supported by mass spectrometry analyses comparing CD11b+ microglia from 6 month old 5xFAD mice to age-matched WT mice and LPS-induced acute inflamed microglia (Rangaraju et al., 2018). Interestingly, besides TREM2 also other AD risk genes were found to be highly expressed by DAM, including Cst7, Ctsb, Lpl, Tyrobp and ApoE (Keren-Shaul et al., 2017; Lambert et al., 2013; Mathys et al., 2017; Paz-y-Miño et al., 2015; Pottier et al., 2016; Scacchi et al., 2004).

\section{DAM features}

Besides more or less effective $A \beta$-clearance, microglia also attempt to shield $A \beta$-plaques from neurons to avoid neuronal toxicity. Therefore, they cluster around $A \beta$-deposits and compressed $A \beta$ peptides into densely packed $A \beta$-plaques. Current data shows that microglia build functional barriers around $A \beta$-plaques to prevent protofibrillary $A \beta_{42}$ hotspots and thereby inhibit further $A \beta$-plaque growth. These findings were verified in two APP overexpressing mouse models (Condello et al., 2015). Additionally, the microglia barrier is considered to avoid axonal dystrophy in the proximate neuropil (Condello et al., 2015). However, another current report showed by in vivo two-photon imaging of 4-7 month old 5XFAD mice that PAM are driven into cell death and thereby release un-degraded $A \beta$-peptides, in particular $A \beta_{42}$, into the extracellular space. This contributes to further $A \beta$-plaque growth by attaching $A \beta_{42}$ to pre-existing $A \beta$-plaques (Baik et al., 2016). Thus, depending amongst others on their physical proximity to $A \beta$-plaques, microglia reveal heterogenic activity and function, which emphasizes the necessity to distinguish PAM and DAM from A $\beta$-plaque distant microglia. 


\section{Inflammation and NLRP3 inflammasome}

Neuroinflammation is a prominent feature of $A D$. The microglial inflammatory response in $A D$ is not only beneficial in recruiting other cells to clear $A \beta$-peptides and apoptotic cells, but it can also contribute to neurotoxicity (von Bernhardi et al., 2015; Wood et al., 2015). In particular, DAM show elevated upregulation of inflammatory gene expression, including IL-1 $\beta$, IL-6, TNF- $\alpha$ (tumor necrosis factor alpha) and iNOS (Jay et al., 2017). Transcription of TNF- $\alpha$ was also rapidly upregulated in early-response to neurodegeneration in DAM of young CK-p25 mice (Mathys et al., 2017). Interestingly, TNF- $\alpha$ can contribute to the activation of an intracellular multiprotein complex, called the NLRP3 (NACHT-, LRR- and pyrin (PYD)-domain-containing protein 3) in microglia, which helps to produce mature IL-1 $\beta$. Typically external stimuli such as activation of TNF-receptors TNFR1 and TNFR2, TLRs and others can induce expression of the NLRP3, pro-IL-1 $\beta$ and pro-IL18 genes through NF-kB (nuclear factor-kappa B) (Bauernfeind et al., 2009; Franchi et al., 2009). This process is termed priming. A second stimulus is needed to effectively activate the NLRP3 inflammasome through assembly of the NLPR3 protein, which builds a molecular sensor, the ASC (adaptor molecule apoptosis-associated speck-like protein containing a CARD) and the effector protease caspase 1 . In $A D$, this second stimulus can be Trem2-dependent signaling but also mediated by lysosomal damage (Amaral et al., 2018; Heneka et al., 2012; Hornung et al., 2008). Accumulation of $A \beta$-peptides in lysosomes due to acidification and dysfunctional degradation was identified to result in lysosomal rupture in AD (Baik et al., 2016; Halle et al., 2008; Heneka et al., 2012; Yang et al., 1998).

The functionally assembled NRLP3 complex then maturates pro-IL- $1 \beta$ into IL- $1 \beta$ and pro-IL-18 into IL-18 through caspase 1. NLRP3 activation can cause programmed cell death called pyroptosis, which results in the release of IL-1 $1 \beta, \mathrm{IL}-18$ and ASC specks into the extracellular space.

In AD, NLRP3 was found to be highly active and to contribute to $A \beta$-pathology as inhibition of the NLRP3 in APP/PS1 mice prevented memory loss, diminished A $\beta$-burden and reduced proinflammatory response of microglia (Heneka et al., 2012). Extracellular ASC specks can be taken up by other microglia and pursue an immune response (Baroja-Mazo et al., 2014; Franklin et al., 2014). In $A D$, these extracellular ASC specks were found to rapidly bind to $A \beta$-peptides, triggering oligomerization and aggregations and thereby induce inflammation-dependent promotion of A $\beta$-plaque seeding (Venegas et al., 2017).

\section{Manipulation of microglia in AD mouse models}

To address whether microglia are beneficial or detrimental for $A D$ progression, elimination of microglia was examined in different AD mouse models.

One of the earliest studies used a ganciclovir-inducible approach to ablate CD11b+ microglia and macrophages. For four weeks APP/PS1 mice were treated via osmotic pumps with ganciclovir to activate the suicide gene HSVTK under control of the CD11b-promoter and to achieve microglia depletion (Grathwohl et al., 2009). Starting ganciclovir treatment at different ages, thus at 
progressed stages of amyloidosis, did not result in changes in the overall $A \beta$-burden. Neither did the authors find diminished neuronal loss (Grathwohl et al., 2009).

A current study achieved microglia ablation in a model with conditional depletion of diphtheria toxinsensoring $\mathrm{CX}_{3} \mathrm{CR} 1+$ cells in APP/PS1 mice. Briefly, i.p. tamoxifen application resulted in the $\mathrm{CX}_{3} \mathrm{CR} 1$-dependent expression of Cre-recombinase. Cre cleaved diphtheria toxin receptor (DTR) from Rosa26-stop-iDTR transgene in a $\mathrm{CX}_{3} \mathrm{CR} 1$-dependent manner. Additional i.p. application of diphtheria toxin then resulted in microglia depletion (Zhao et al., 2017). Microglia ablation at 12 and 24 month old mice did not reduce $A \beta$-plaque numbers but led to moderate growth of $A \beta$-plaques within one week after microglia depletion as monitored by in vivo two-photon imaging (Zhao et al., 2017).

Other groups investigated microglia depletion on AD progression by pharmacological inhibition of CSF1R, which resulted in almost complete microglia ablation in 3xTg, APP/PS1 mice and 5xFAD mice (Dagher et al., 2015; Olmos-Alonso et al., 2016; Spangenberg et al., 2016). Olmos-Alonso and colleagues (2016) used the pharmacological inhibitor GW2580 as chow supplement on 6 month old APP/PS1 mice for a duration of 3 months. After treatment these mice revealed reduced inflammatory phenotypes, improved memory and behavioral performances, reduced synaptic degeneration while $A \beta$-load was not altered. Another study used 1 month treatment with the dual CSF1R/c-kit inhibitor PLX3397 (600 mg/kg) to deplete microglia in 5xFAD. Treatment was performed in 2 month, 10 month and 14 month old animals, respectively (Spangenberg et al., 2016). Again, microglia elimination did not improve $A \beta$-load. However, Golgi-Cox analysis revealed reduced loss of dendritic spines and cognitive abilities were restored as measured by contextual fear conditioning in the 10 month cohort. The study carried out by Dagher and colleagues (2015) found cognitive improvement upon inhibited microglia proliferation with low concentrations of PLX3397 (300 mg/kg) in 15 month old 3XTg mice. These mice harbor the Swedish mutation in human APP, M146V mutation in PSEN1 and additionally a P301L mutation in the MAPT gene and therefore develop A $\beta$ - and Tau-pathology at the age of 15 months (Oddo et al., 2004). Already short-term treatment for 6 weeks with low concentrations of PLX3397 resulted in significant cognitive improvement validated by novel object recognition, while $A \beta$-burden and the load of pTau was not changed (Dagher et al., 2015).

Given that Trem2 is crucial for phago-lysosomally active DAM formation in AD, it is important to know that Trem2 was identified to synergize with CSF1R signaling and thereby promote microglial survival (Wang et al., 2015b). 5xFAD mice with Trem2 KO could not migrate towards A $\beta$-plaques and resulted in more diffuse $A \beta$-plaques (Wang et al., 2015b). Strikingly, Trem2 overexpression was able to extenuate neurite dystrophy and improve cognitive performance in 5xFAD mice (Lee et al., 2018). 


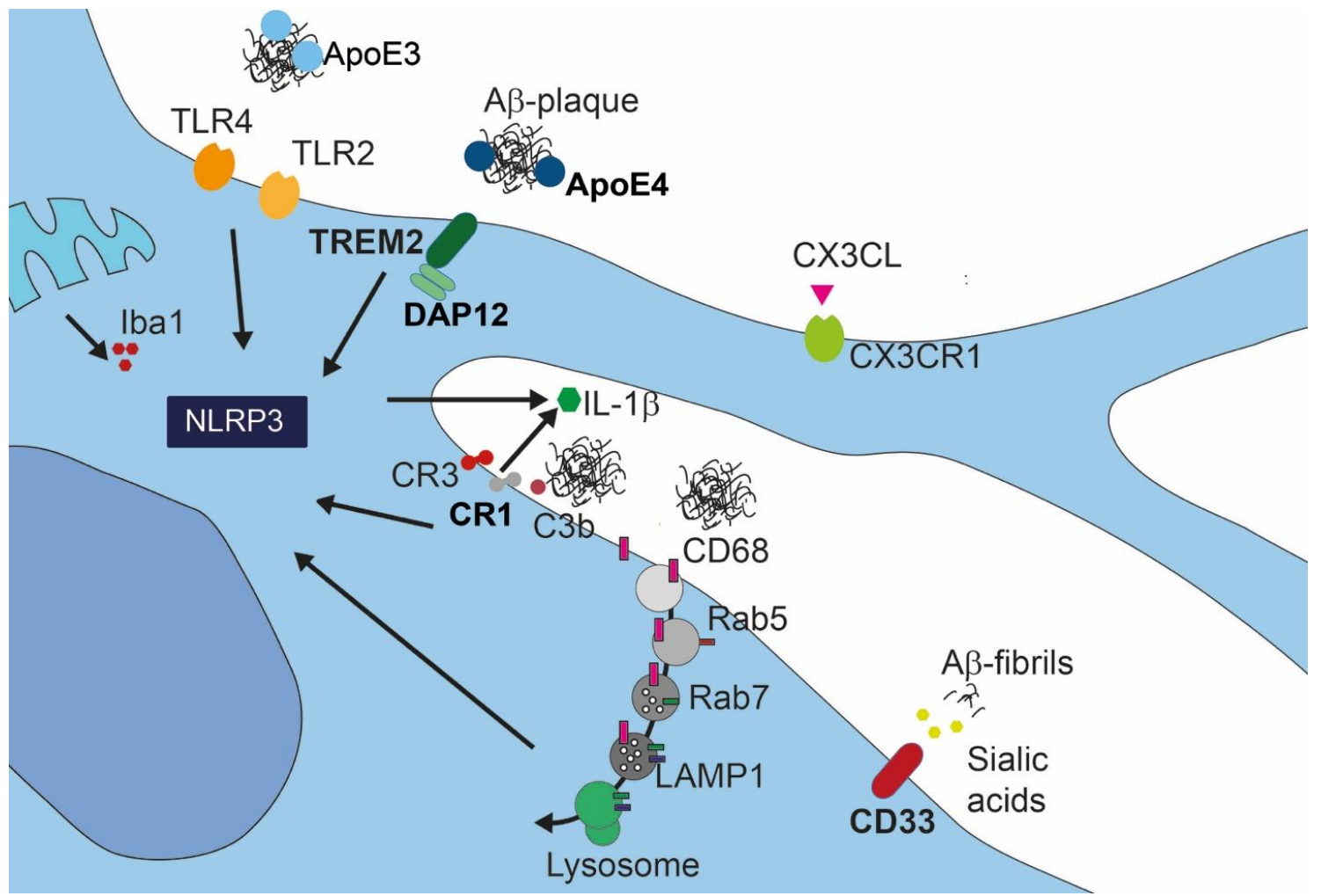

Figure 9: Expression of AD risk genes and endo-lysosomal degradation in microglia.

Scheme of several signaling pathways in microglia, upregulated in AD. This includes Toll-like receptors TLR2 and TLR4, CD33 (also referred to as Siglec-3, sialic acid-binding immunoglobulin-like lectin), complement factor C3b and complement receptors CR1 and CR3, TREM2 (triggering receptor expressed in myeloid cells 2) and its downstream compound DAP12 (DNAX-activating protein), fractalkine receptor $\mathrm{CX}_{3} \mathrm{CR} 1$ and its ligand $\mathrm{CX}{ }_{3} \mathrm{CL}$. Apoproteins $\mathrm{ApoE} 3$ and $A p o E 4$ are involed in mediating $A \beta$-phagocytosis. Molecules in bold are identified late-onset $A D$ risk genes. Also markers for the endo-lysosomal pathway such as LAMP1, CD68 and Rab7 were shown to be upregulated in AD. Receptor-dependent signaling but also lysosomal rupture can activeate the NLRP3 inflammasome which is one source of mature IL-1ß. Iba1 (ionized calcium-binding adapter molecule 1) is cytosolic marker for microglia and CNS macrophages. Adapted from Mosher and Wyss-Coray (2014).

\subsubsection{Rab7-dependent degradation pathways}

Impaired protein degradation is associated with various diseases, including neurodegeneration (Lee et al., 2010). In post mortem tissue of AD patients and in AD mouse models upregulation of proteins associated with endo-lysosomal pathway (Ba et al., 2017; Ginsberg et al., 2010a; Ginsberg et al., 2011; Ginsberg et al., 2010b; Holtman et al., 2015; Nuriel et al., 2017) and autophagy have been identified (Hara et al., 2006; Komatsu et al., 2006).

Whereas the endo-lysosomal pathway is mostly associated with degradation of internalized material, which was engulfed by phagocytosis, (macro-)autophagy is widely responsible for recycling of cellular components including misfolded proteins (Luzio et al., 2007). Both pathways have a common terminal point in fusion with the lysosome, which is a catabolic cell organelle, 
responsible for enzymatic degradation of external and internal debris in eukaryotic cells. This fusion is mediated by Rab7 GTPase activity (Nakamura and Yoshimori, 2017).

\section{Endo-lysosomal degradation}

Early endosomes mature to late endosomes characterized by an exchange of surface molecules and gradual acidification (Figure 10). One typical marker of early endosomes is the GTPase Rab5, which is responsible for proper endosome formation (Hutagalung and Novick, 2011). Along endocytic trafficking and vesicle maturation, Rab5 is replaced by Rab7 marking the switch from early to late endosomes (Feng et al., 1995). Rab7 is expressed on matured, late endosomes and lysosomes (Wandinger-Ness and Zerial, 2014) (Figure 10).

\section{Autophagy}

In a first step the target components are encaged by a membrane known as phagophore. With the aid of several proteins such as microtubule-associated protein 1 light-chain 3 (LC3 protein) this membrane is closed and termed early autophagosome (Kabeya et al., 2000). This formation is amongst others induced by mTor signaling (Kaur and Debnath, 2015). Along maturation Rab7 is expressed on the membrane surface of late endosomes, where it is responsible for the fusion to the lysosome (Gutierrez et al., 2004; Jäger et al., 2004)(Figure 10).

\section{Rab7}

Rab7 belongs to the family of small Ras GTPases, which comprises more than 60 proteins in humans and which are involved in vesicle motility (Kiral et al., 2018). In general, Rab proteins shuttle between the cytosol and the membrane to reversibly regulate protein assembly at the membrane by hydrolysis of GTP to GPD (Hutagalung and Novick, 2011).

In interaction with motorproteins like kinesin and dynein, Rab7 is involved in the intracellular transport of late endosomes /autophagosomes and lysosomes along the cytoskeleton and therefore is considered a key factor of the endocytic pathway (Cai et al., 2010; Edinger et al., 2003; Gutierrez et al., 2004; Jager et al., 2004). Moreover, Rab7 is important for the biogenesis and maintenance of lysosomes and finally, for the fusion of late endosomes / late autophagosomes to the lysosome within the perinuclear compartment (Bucci et al., 2000). At this stage of fusion, resulting in endolysosomes / autophagosome-lysosome hybrids, markers like LAMP1 and LAMP2 are detectable. Alongside the maturation process the $\mathrm{pH}$ within the vesicle is gradually shifted from neutral to acidic $\mathrm{pH}$ by proton pumps. Considering that Rab7 is involved in the maturation of endosomes as well as in the fusion process with the lysosome, this protein is of great importance for proper protein degradation. Hence, it is not surprising that mutations of Rab7 underlie severe diseases. For instance, mutations within Rab7 were identified in genetic inherited Charcot-Marie-Tooth type 2B disease, which is characterized by neurodegeneration of somatosensory neurons (McCray et al., 


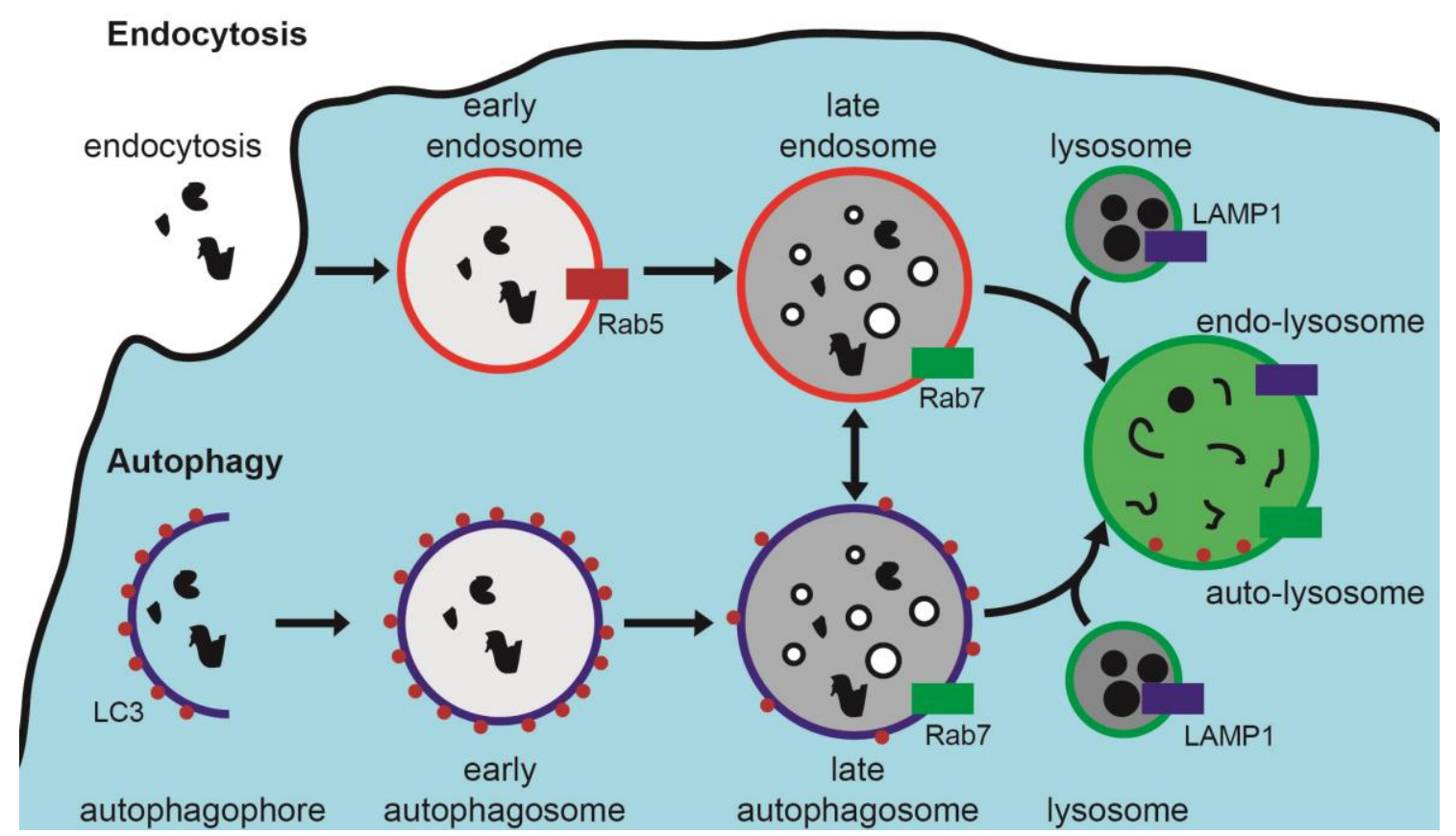

Figure 10: Schematic role of Rab7 on endo-lysosomal degradation and autophagy.

Top: In the first step of the endocytic pathway, extrinsic debris, pathogens or other molecules are internalized by endocytosis and caged in a Rab5+ early endosome. This vesicle maturates and Rab5 (red) is replaced by Rab7 (green) which labels the late endosome. By fusion with the lysosome the endo-lysosome hybrid is formed which is also positive for LAMP1 (blue). Bottom: To degrade internal material, an autophagophore is built up by membrane components and LC3 (microtubule associated protein 1 light chain3; red). As the membranes closes up to a vesicle, it is named early autophagosome. Throughout maturation Rab7 is expressed, which helps in fusion with the lysosome. Noteworthy, late endosomes and late autophagosomes can also fuse with each other and generate the so-called amphisome, which in turn fuses to the lysosome. Both pathways go along gradual acidification also vesicle maturation. Within the final step lysosomal enzymes are released which degrade the cargo in a pH-dependent manner. Adapted from Hyttinen et al., (2017).

2010; Verhoeven et al., 2003). This emphasizes the crucial role of Rab7 function on neuronal homeostasis.

\subsubsection{Impact of the endosomal-lysosomal pathway on AD}

Amongst the earliest findings in $A D$ are enlarged endosomal compartments and accumulated phagocytic vacuoles in neurons (Cataldo et al., 2008; Cataldo et al., 2000; Nixon et al., 2005; Yang et al., 2011). One explanation for this is the imbalanced production and clearance of A $\beta$-peptides, which can also be a consequence of impairments in the ubiquitin-proteasomal pathway (Gregori et al., 1995; Tseng et al., 2008). Due to this imbalance in A $\beta$-production, proteins accumulate and become too large for ubiquitin-proteasomal degradation, and therefore require protein degradation through either autophagy or endo-lysosomal pathways.

In terms of autophagy, mutations in PSEN1 contribute to elevated autophagosome enlargement and accumulation of autophagosomes within dystrophic neurites resulting in neuronal cell death (Boland et al., 2008; Lee et al., 2010; Yu et al., 2010). Genetic depletion of autophagy inhibitor 
cystatin $b$ restored autophagic function, and was able to prevent cognitive impairment while reducing A $\beta$-load in TgCRND8 mice (Yang et al., 2011).

Besides autophagy, the endocytic pathway was found to be defective in AD. Severity and progression of $\mathrm{MCl}$ and $\mathrm{AD}$ were found to correlate with Rab5+ and Rab7+ endocytic vesicles, which are highly abundant in cholinergic neurons in brain regions with enriched pTau levels (Ginsberg et al., 2010a; Ginsberg et al., 2011; Ginsberg et al., 2010b). Moreover, human CSF samples of AD patients showed elevated levels of late endosomal markers like Lamp1, Lamp2 and Rab7 (Armstrong et al., 2014). On a functional level, inhibition of lysosomal proteolysis was shown to induce axonal dystrophy in neurons (Lee et al., 2011).

GWAS analyses of patients with late onset AD identified risk genes associated with endo-lysosomal pathway, such as Sortilin Related Receptor 1 (SORL1), Phosphatidylinositol Binding Clathrin Assembly Protein (PICALM) and Ras And Rab Interactor 3 (RIN3) (Hollingworth et al., 2011; Lambert et al., 2009; Lambert et al., 2013). Surprisingly, whole-exome sequencing of early onset AD patients, who were not carrying mutations in APP, PSEN1 and PSEN2 or allele variants of known late-onset $A D$ risk genes, identified candidate $A D$ risk genes in the endo-lysosomal transport (Kunkle et al., 2017). Shared risk genes found in early and late onset AD samples not only included endo-lysosomal genes SORL1, RIN3, but also Trem2 and MHCII, which are associated with myeloid cells. Since microglia are the main phagocytes of the brain, it is not surprising that several identified overlapping genes are expressed in microglia.

\section{Microglia}

Concurrently, microglia show inefficiency of degradation activity through autophagy and endolysosomal pathway in AD (e.g. Gosselin et al., 2017; Landel et al., 2014; Nuriel et al., 2017). Moreover, several risk genes for sporadic AD such as ApoE, Trem2 or DAP12 were found to induce expression of molecules involved in the endo-lysosomal pathway of myeloid cells (Hollingworth et al., 2011; Lambert et al., 2009; Lambert et al., 2013). This includes CD68 which is a myeloid cell specific transmembrane glycoprotein and is expressed along all stages of the endo-lysosomal pathway (Figure 10). CD68 belongs to the scavenger receptor family, which is associated with phagocytosis and protein degradation. In the CNS, it is often used as a marker for microglia activation and is highly abundant in AD brain tissue (Minett et al., 2016).

As mentioned, Trem2 is responsible for the transcriptional change of microglia towards DAM. Trem2 KO in 5xFAD mice triggered elevated autophagy by suppressing mTor signaling in microglia (Ulland et al., 2017). Similarly, Rab7 KO in vitro experiments unveiled enhanced autophagosome formation in an mTOR1 independent pathway (Kuchitsu et al., 2018). Pharmacological and genetic disruption of autophagosome formation in myeloid cells of $A D$ transgenic mice improve cognitive functions and diminished A $\beta$-pathology in these mice (Caccamo et al., 2010; Cho et al., 2014; Kim et al., 2017; Spilman et al., 2010). These findings are comparable to studies investigating 
phagocytosis in AD by inhibiting complement factors (Fonseca et al., 2004; Hong et al., 2016; Paolicelli et al., 2011; Shi et al., 2017).

Transgenic mice overexpressing human ApoE4, which is upstream of Trem2, revealed transcriptional upregulation of various genes of the endosomal-lysosomal pathway including Rab7, while showing enlarged endosomes in $A D$ affected brain regions compared to non-affected ones (Nuriel et al., 2017). In contrast pretreatment of primary murine microglia with the benign variant ApoE3 increased $A \beta$-clearance by enhancing the recycling of Rab7+ endosomes from the lysosome back to the early endosome (Lee et al., 2012).

Microglial cytokines IL-6 and IL-12 can directly modulate the endo-lysosomal pathway in vitro. While IL-6 can directly induce Rab5 expression and IL-12 activated Rab7 expression in the murine J774E monocyte cell line (Bhattacharya et al., 2006). Therefore, microglial autophagy and endo-lysosomal degradation seem of great importance for amyloidogenesis and AD development. Since Rab7 is crucial for the fusion with the lysosome in both of these pathways, this GTPase is a good target to evaluate lysosomal degradation on in microglia on $A D$ pathology.

\subsubsection{Targeting microglial endo-lysosomal degradation in vivo: the Rab7 ${ }^{\Delta M G}$ mouse line}

The myeloid lineage shares numerous features, such as the expression of similar genes. Thus, it is very difficult to specifically target a subset of this lineage, such as microglia. However, taking advantage of the fact that microglia do not renew by differentiation from hematopeotic precursor cells in adults, Yona and co-workers (2013) have created a tamoxifen inducible Cre-line which expresses Cre-recombinase under transcriptional control of the monocyte-specific $\mathrm{CX}_{3} \mathrm{CR} 1$ promoter. $\mathrm{CX}_{3} \mathrm{CR} 1$ is a Gai-coupled seven-transmembrane receptor prominently expressed by microglia and other cells of the myeloid lineage, but also found a subset of $T$ cells and natural killer cells (NKs) (Bazan et al., 1997; Imai et al., 1997; Pan et al., 1997). CX ${ }_{3}$ CL1-CX ${ }_{3}$ CR1 interaction is crucial for phagocytic removal of synaptic debris during synaptic pruning (Paolicelli et al., 2011). $\mathrm{CX}_{3} \mathrm{CL}$ is expressed by a subset of neurons (Hughes et al., 2002).

Due to the fact that microglia do not differentiate from hematopoetic stem cells throughout adulthood, in contrary to other cells of the myeloid lineage, and can maintain themselves through

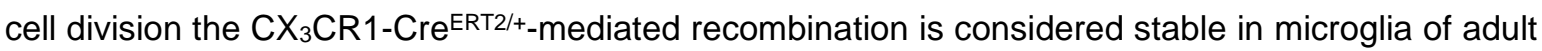
mice (Figure 11). Moreover, other $\mathrm{CX}_{3} \mathrm{CR} 1$-expressing cells undergo physiological turnover and thereby gradually loose recombination, which is mostly erased in Ly6+ blood monocytes and intestinal macrophages two months after tamoxifen administration (Yona et al., 2013). Crossbreeding of the $\mathrm{CX}_{3} \mathrm{CR}$-Cre ${ }^{\mathrm{ERT} /+}$ line with a YFP-reporter line verified an efficiency of more than $90 \%$ recombination in microglia (Yona et al., 2013). 
The Cre-recombination and thereby the mediated $\mathrm{KO}$ in other $\mathrm{CX}_{3} \mathrm{CR} 1$-expressing cells of the myeloid cell lineage is not detectable. It must be mentioned though, that there are other myeloid cell types, such as Kupffer cells in the liver, which are also established before birth and are maintained by self-renewal. However, these cells lack CX ${ }_{3} \mathrm{CR} 1$ expression (Schulz et al., 2012; Yona et al., 2013).

Noteworthy, in the meantime TMEM119 was suggested to be a microglia specific molecule. However due to their heterogeneity, it cannot be ruled out yet that it is only expressed on a subset of microglia (Bennett et al., 2016). Also, there is still no in vivo model accessible that uses a Tmem119-Cre driver. Therefore, as of today, the $\mathrm{CX}_{3} \mathrm{CR}$-Cre ${ }^{E R T 2 /+}$ line is a unique tool to specifically investigate genetic KO within microglia in adulthood.

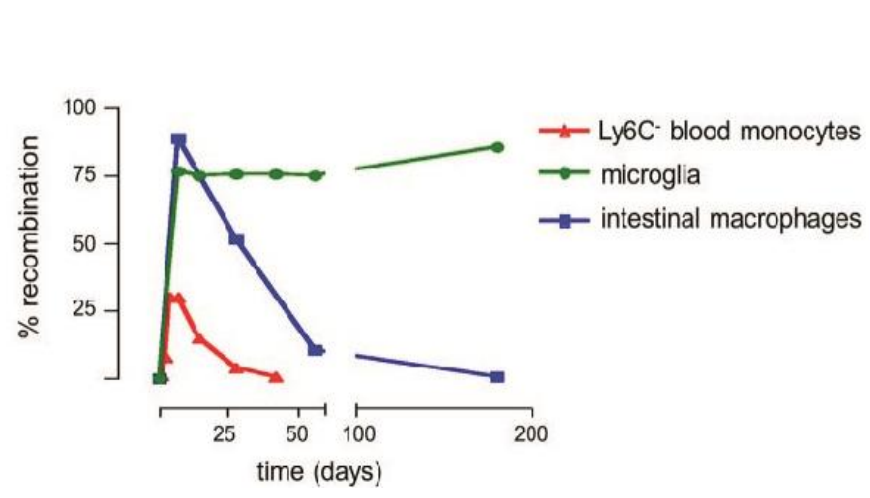

Figure 11: Recombination rate of $\mathrm{CX}_{3} \mathrm{CR} 1$ $\mathrm{Cre}^{\mathrm{ERT} 2 /+}$.

Recombination rates [\%] of $\mathrm{CX}_{3} \mathrm{CR} 1-\mathrm{Cre}^{\mathrm{ERT} 2 /+} \mathrm{x}$ R26-YFP mice after tamoxifen application in different myeloid cell types. Throughout time other $\mathrm{CX}_{3} \mathrm{CR} 1$-expressing cells like Ly6C+ blood monocytes (red) and intestinal macrophages (blue) loose gene rearranngement due to physiological turnover. Microglia (green) show persisting recombination also 200 days after tamoxifen administration. Figure adapted form Wolf et at (2013).

To achieve microglia-specific disruption of endo-lysosomal function, the $\mathrm{CX}_{3} \mathrm{CR}$-Cre ${ }^{\mathrm{ERT} 2 /+}$ line was already successfully crossed to the Rab7 f//fl mouse line which harbors loxP sites (il) flanking exon 2 of the Rab7 gene (Roy et al., 2013; Safaiyan et al., 2016). By application of tamoxifen transcription of Cre-recombinase in $\mathrm{CX}_{3} \mathrm{CR} 1+$ cells was achieved and resulted in the effective $\mathrm{KO}$ of Rab7 in microglia (Rab7 $\triangle \mathrm{MG}$ ), which becomes microglia-exclusive through aging (Safaiyan et al., 2016).

To elucidate the impact of microglia Rab7-mediated endo-lysosomal function on A $\beta$-pathology in vivo, we have crossed these mice to 5xFAD mice which were presented in 1.1.4. 


\subsection{Aim of this study}

There is much evidence pointing towards a crucial role of microglia, the main immune cells of the brain, on disease pathology in $A D$. It is not clear, whether microglia are beneficial by degrading toxic $A \beta$-species or whether they contribute to disease by secondary inflammatory activation.

This study aims to elucidate the role of microglial clearance function on neuroinflammation and amyloid pathology in vivo. We hypothesize that microglia with defective endo-lysosomal pathway fail to degrade amyloid and thereby have stronger amyloid pathology compared to control mice.

Therefore, triple transgenic mice with a microglia-specific $\left(\mathrm{CX}_{3} \mathrm{CR} 1-\mathrm{Cre}^{\mathrm{ERT2/+}}\right)$ knockout of the Rab7 allele $\left(R a b 7^{f / f \mid l}\right)$ were generated. These mice were further crossed with the 5xFAD mouse model to investigate the impact of the endo-lysosomal pathway in microglia on AD progression.

The specific questions that my project addressed are:

1. Does the knockout of Rab7 affect microglial amyloid uptake and degradation?

2. Does the microglial disruption of the endosomal-lysosomal pathway affect amyloid burden, neuronal loss, synaptic loss and neuroinflammation?

3. Does the microglial knockout of Rab7 induce compensatory mechanisms by recruiting other cell types in the 5xFAD mouse model? 


\section{Materials and Methods}

\subsection{Materials}

\subsubsection{Buffers and Solutions}

All buffers and solution were prepared with autoclaved double-distilled water $\left(\mathrm{ddH}_{2} \mathrm{O}\right)$ and stored at room temperature (RT), unless explicitly declared otherwise. Chemicals were purchased from Merck Millipore (Darmstadt, Germany), Roth (Karlsruhe, Germany) or Sigma-Aldrich (München, Germany) unless stated otherwise. Plastic consumables were purchased from BD Falcon ${ }^{\mathrm{TM}}$ (BD Biosciences, Le Pont de Claix, France), Eppendorf AG (Hamburg, Germany) and Greiner bio-One GmbH (Frickenhausen, Germany). Glass slides and cover slips for microscopy were purchased from Jung HistoService (Nussloch, Germany).

\subsubsection{General buffers}

Table 1: Summary of used buffers and solutions in this thesis.

\begin{tabular}{|c|c|c|}
\hline Buffer & Composition & \\
\hline $0.1 \mathrm{M}$ Phosphate buffer (pH 7.2): & $\begin{array}{l}27 \mathrm{mM} \\
77 \mathrm{mM} \\
300 \mathrm{mM}\end{array}$ & $\begin{array}{l}\mathrm{NaH}_{2} \mathrm{PO}_{4}{ }^{*} \mathrm{H}_{2} \mathrm{O} \\
\mathrm{Na}_{2} \mathrm{HPO}_{4} \\
\mathrm{NaCl}\end{array}$ \\
\hline $\begin{array}{l}\text { 10x Phosphate buffered saline (PBS; } \\
\text { pH 7.4): }\end{array}$ & $\begin{array}{l}1.35 \mathrm{M} \\
27 \mathrm{mM} \\
100 \mathrm{mM} \\
18 \mathrm{mM}\end{array}$ & $\begin{array}{l}\mathrm{NaCl} \\
\mathrm{KCl} \\
\mathrm{Na}_{2} \mathrm{HPO}_{4} \\
\mathrm{KH}_{2} \mathrm{PO}_{4}\end{array}$ \\
\hline Tail lysis buffer: & $\begin{array}{l}100 \mathrm{mM} \\
5 \mathrm{mM} \\
200 \mathrm{mM} \\
0.2 \%[\mathrm{w} / \mathrm{v}]\end{array}$ & $\begin{array}{l}\text { Tris } \mathrm{HCl}, \mathrm{pH} 8.5 \\
\text { EDTA } \\
\mathrm{NaCl} \\
\text { SDS }\end{array}$ \\
\hline $\begin{array}{l}\text { 10x TRIS-Borat-EDTA (TBE) buffer } \\
\text { (pH 8.0): }\end{array}$ & $\begin{array}{l}840 \mathrm{mM} \\
890 \mathrm{mM} \\
20 \mathrm{mM}\end{array}$ & $\begin{array}{l}\text { Tris Base } \\
\text { Boric Acid } \\
\text { EDTA }\end{array}$ \\
\hline 10x Tris buffered saline (TBS): & $\begin{array}{l}1.37 \mathrm{M} \\
27 \mathrm{mM} \\
248 \mathrm{mM}\end{array}$ & $\begin{array}{l}\mathrm{NaCl} \\
\mathrm{KCl} \\
\text { Tris-HCl}\end{array}$ \\
\hline
\end{tabular}

Working solutions like $1 x$ PBS and $1 x$ TBS were used as $10 \%[v / v] 10 x$ stock solutions in $\mathrm{ddH}_{2} \mathrm{O}$. 


\subsubsection{Buffers for Protein Biochemistry}

Table 2: Buffers for protein biochemistry.

\begin{tabular}{|c|c|c|}
\hline Buffer & \multicolumn{2}{|l|}{ Composition } \\
\hline 1x CHAPS buffer (pH 8.0): & $\begin{array}{l}16 \mathrm{mM} \\
39 \mathrm{mM} \\
5 \mathrm{mM}\end{array}$ & $\begin{array}{l}\text { Chaps } \\
\text { Tris-HCl } \\
\text { EDTA }\end{array}$ \\
\hline Homogenization buffer: & $\begin{array}{l}1 \mathrm{x} \\
1 \mathrm{mM} \\
1 \mathrm{mM} \\
3 \mu \mathrm{l} / \mathrm{ml} \\
3 \mu \mathrm{l} / \mathrm{ml}\end{array}$ & $\begin{array}{l}\text { PBS } \\
\text { EDTA } \\
\text { EGTA } \\
\text { protease inhibitor mix } \\
\text { phosphatase inhibitor I+II }\end{array}$ \\
\hline 2x RIPA: & $\begin{array}{l}50 \mathrm{mM} \\
1 \%[\mathrm{w} / \mathrm{v}] \\
0.5 \%[\mathrm{w} / \mathrm{v}] \\
0.1 \%[\mathrm{w} / \mathrm{v}] \\
150 \mathrm{mM} \\
2 \mathrm{mM} \\
50 \mathrm{mM}\end{array}$ & $\begin{array}{l}\text { Tris } \mathrm{HCl}, \mathrm{pH} 7.4 \\
\mathrm{NP}-40 \\
\text { Sodiumdeoxycholate } \\
\text { SDS } \\
\mathrm{NaCl} \\
\text { EDTA } \\
\mathrm{NaF}\end{array}$ \\
\hline 0.025\% PBST: & $\begin{array}{l}0.025 \%[\mathrm{v} / \mathrm{v}] \\
1 \mathrm{x}\end{array}$ & $\begin{array}{l}\text { Tween-20 } \\
\text { PBS }\end{array}$ \\
\hline 10x Running Buffer: & $\begin{array}{l}1.9 \mathrm{M} \\
192 \mathrm{mM} \\
1 \%[\mathrm{w} / \mathrm{v}]\end{array}$ & $\begin{array}{l}\text { Glycine } \\
\text { Tris-HCl } \\
\text { SDS }\end{array}$ \\
\hline Sample Buffer: & $\begin{array}{l}312.5 \mathrm{mM} \\
5 \mathrm{mM} \\
10 \%[\mathrm{w} / \mathrm{v}] \\
0.05 \%[\mathrm{w} / \mathrm{v}] \\
50 \%[\mathrm{v} / \mathrm{v}] \\
0.5 \%[\mathrm{v} / \mathrm{v}] \\
\text { Long-term stc } \\
\text { working aliqu }\end{array}$ & $\begin{array}{l}\text { Tris- } \mathrm{HCl}, \mathrm{pH} 6.8 \\
\text { EDTA, pH } 8.0 \\
\text { SDS } \\
\text { Bromphenol blue } \\
\text { Glycerol } \\
\beta-\text { Mercaptoethanol } \\
\text { at }-20^{\circ} \mathrm{C} \text {; } \\
\text { red at } 4^{\circ} \mathrm{C}\end{array}$ \\
\hline \multirow[t]{2}{*}{ 10x Transfer Buffer: } & $\begin{array}{l}1.9 \mathrm{M} \\
192 \mathrm{mM}\end{array}$ & $\begin{array}{l}\text { Glycine } \\
\text { Tris- } \mathrm{HCl}\end{array}$ \\
\hline & \multicolumn{2}{|c|}{$\begin{array}{l}\text { For } 1 \times \text { Transfer buffer the } 1 \text { part of the } 10 x \text { stock was } \\
\text { mixed with } 2 \text { parts methanol and } 7 \text { parts } \mathrm{ddH}_{2} \mathrm{O}\end{array}$} \\
\hline Resolving Buffer (pH 6.8): & $0.5 \mathrm{M}$ & Tris- $\mathrm{HCl}$ \\
\hline Stacking Buffer (pH 8.8): & $1.5 \mathrm{M}$ & Tris-HCl \\
\hline
\end{tabular}




\subsubsection{Buffers for Histology}

Table 3: Buffers for histology.

\begin{tabular}{|c|c|c|}
\hline Buffer & Composition & \\
\hline Avertin: & $\begin{array}{l}72 \mathrm{mM} \\
2 \%[\mathrm{v} / \mathrm{v}] \\
\text { Dissolved at } 40^{\circ} \mathrm{C} \\
\text { Stored at }-20^{\circ} \mathrm{C}, \text { lig }\end{array}$ & $\begin{array}{l}\text { 2,2,2 Tribromethanol } \\
\text { tert-amylalcohol } \\
\text { ht protected }\end{array}$ \\
\hline 100\% blocking solution: & $\begin{array}{l}2.5 \%[\mathrm{v} / \mathrm{v}] \\
2.5 \%[\mathrm{w} / \mathrm{v}] \\
2.5 \%[\mathrm{v} / \mathrm{v}] \\
1 \mathrm{x} \\
\text { Stored at }-20^{\circ} \mathrm{C}\end{array}$ & $\begin{array}{l}\text { FBS } \\
\text { BSA } \\
\text { fish gelatin } \\
\text { PBS }\end{array}$ \\
\hline Citric buffer: & $\begin{array}{l}11 \mathrm{mM} \\
0.05 \%[\mathrm{v} / \mathrm{v}] \\
\text { Used for up to } 3 \mathrm{mc}\end{array}$ & $\begin{array}{l}\text { Tri-Sodium Citrate } \\
\text { Tween-20 } \\
\text { onths }\end{array}$ \\
\hline Cryoprotective medium: & $\begin{array}{l}25 \%[v / v] \\
25 \%[v / v] \\
1 x\end{array}$ & $\begin{array}{l}\text { Glycerol } \\
\text { Ethyleneglycol } \\
\text { PBS }\end{array}$ \\
\hline Golgi-Cox Stock solution 1: & $5 \%[w / v]$ & $\begin{array}{ll}\text { Potassium dichromate } & \begin{array}{l}\text { Stored in the } \\
\text { dark at RT }\end{array}\end{array}$ \\
\hline Golgi-Cox Stock solution 2: & $5 \%[w / v]$ & Mercuric chloride \\
\hline Golgi-Cox Stock solution 3: & $5 \%[w / v]$ & Potassium chromate \\
\hline Golgi-Cox Solution: & $\begin{array}{l}24 \%[\mathrm{w} / \mathrm{v}] \\
24 \%[\mathrm{w} / \mathrm{v}] \\
19.2 \%[\mathrm{w} / \mathrm{v}] \\
\text { Mixed at least } 48 \mathrm{~h} \\
\text { the bottom of the b } \\
\text { Stored at RT, in a v } \\
\text { stable for up to } 3 \mathrm{~m}\end{array}$ & $\begin{array}{l}\text { Potassium dichromate (Stock solution 1) } \\
\text { Mercuric chloride (Stock solution 2) } \\
\text { Potassium chromate (Stock solution 3) } \\
\text { prior to use to allow precipitates to sink to } \\
\text { ottle. } \\
\text { vell-ventilated, light-protected environment } \\
\text { lonths }\end{array}$ \\
\hline $\begin{array}{l}\text { Golgi-Cox Tissue Protectant } \\
\text { Solution: }\end{array}$ & $\begin{array}{l}50 \%[\mathrm{v} / \mathrm{v}] \\
33.3 \%[\mathrm{w} / \mathrm{v}] \\
90 \mathrm{mM} \\
33.3 \%[\mathrm{v} / \mathrm{v}] \\
\text { Stored at } 4^{\circ} \mathrm{C} \text {, in th }\end{array}$ & $\begin{array}{l}0.1 \mathrm{M} \text { Phosphate buffer (pH 7.2) } \\
\text { Sucrose } \\
\text { Polyvinylpyrrolidone } \\
\text { Ethylene glycol } \\
\text { e dark }\end{array}$ \\
\hline Mowiol: & $\begin{array}{l}13.3 \%[\mathrm{w} / \mathrm{v}] \\
33.3 \%[\mathrm{v} / \mathrm{v}] \\
133 \mathrm{mM} \\
24 \mathrm{mg} / \mathrm{ml}\end{array}$ & $\begin{array}{l}\text { Mowiol } \\
\text { Glycerol } \\
\text { Tris HCl, pH } 8.5 \\
\text { DABCO }\end{array}$ \\
\hline 16\% paraformaldehyd (PFA): & $\begin{array}{l}16 \%[\mathrm{w} / \mathrm{v}] \\
1 \mathrm{x} \\
\text { Dissolved at } 55^{\circ} \mathrm{C} \\
\text { Adjust } \mathrm{pH} \text { to } 7.4 \\
\text { Stored at }-20^{\circ} \mathrm{C} \\
\text { Diluted to } 4 \% \text { PFA }\end{array}$ & $\begin{array}{l}\text { Paraformaldehyd } \\
\text { PBS }\end{array}$ \\
\hline
\end{tabular}




$\begin{array}{lll}0.1 \% \text { TBST: } & 0.1 \%[\mathrm{v} / \mathrm{v}] & \text { Tween-20 } \\ & 1 \mathrm{x} & \text { TBS }\end{array}$

\subsubsection{Commercial kits, compounds and consumables}

Table 4: Commercial kits.

\begin{tabular}{lll}
\hline \hline Kit & Catalog number & Company \\
\hline \hline Pierce $^{\mathrm{TM}}$ BCA Protein Assay Kit & 23225 & Thermo Fisher \\
\hline $\begin{array}{l}\text { V-PLEX A Peptide Panel 1 (6E10) } \\
\text { Kit }\end{array}$ & K15200E-2 & $\begin{array}{l}\text { Meso Scale Discovery, } \\
\text { Rockville MD, USA }\end{array}$ \\
\hline \hline
\end{tabular}

Table 5: List of compounds and consumables.

\begin{tabular}{|c|c|c|}
\hline Solution/Medium & Catalog number & Company \\
\hline $\begin{array}{l}\text { Amersham }{ }^{\mathrm{TM}} \mathrm{ECL} \text { Prime Western } \\
\text { Blotting Detection Reagent }\end{array}$ & GERPN2232 & $\begin{array}{l}\text { GE Healthcare Life Sciences, } \\
\text { Little Chalfont, United Kingdom }\end{array}$ \\
\hline $\begin{array}{l}\text { cOmplete }{ }^{\mathrm{TM}} \text { Protease inhibitor } \\
\text { cocktail }\end{array}$ & $000 Z 00011697498001$ & $\begin{array}{l}\text { Roche Diagnostics, Rotkreuz, } \\
\text { Switzerland }\end{array}$ \\
\hline Corn oil & C8267-500ML & $\begin{array}{l}\text { Sigma-Aldrich, Munich, } \\
\text { Germany }\end{array}$ \\
\hline Donkey serum & S2170-500 & $\begin{array}{l}\text { VWR International, Langenfeld, } \\
\text { Germany }\end{array}$ \\
\hline $\begin{array}{l}\text { ECL Western Blotting Substrate } 1 \\
\text { and } 2\end{array}$ & 32106 & $\begin{array}{l}\text { Thermo Fisher Scientific } \\
\text { Inc,Rockford, IL, USA }\end{array}$ \\
\hline Eukitt hard-drying medium & 03989 & $\begin{array}{l}\text { Fluka Analytical, Munich, } \\
\text { Germany }\end{array}$ \\
\hline Gel Red & 41003 & $\begin{array}{l}\text { VWR International, Langenfeld, } \\
\text { Germany }\end{array}$ \\
\hline $\begin{array}{l}\text { GeneRuler } 100 \mathrm{bp} \text { Plus DNA Ladder, } \\
\text { ready-to-use- } 50 \mu \mathrm{g}\end{array}$ & SM0323 & $\begin{array}{l}\text { Fermentas, St. Leon- Rot, } \\
\text { Germany }\end{array}$ \\
\hline GoTaq G2 Flexi DNA Polymerase & M7801 & Promega, Mannheim, Germany \\
\hline $\begin{array}{l}\text { HyClone }^{\mathrm{TM}} \text { Fetal Bovine Serum } \\
\text { (FCS), South American Origin }\end{array}$ & 13567670 & $\begin{array}{l}\text { GE Healthcare Life Sciences, } \\
\text { Little Chalfont, United Kingdom } \\
\text { and Gibcoß, Thermo Fisher } \\
\text { Scientific, Waltham, MA, USA }\end{array}$ \\
\hline Immersol $518 \mathrm{~F}$ & $444960-0000-000$ & Zeiss, Oberkochen, Germany \\
\hline $\begin{array}{l}\text { PageRuler Plus Prestained Protein } \\
\text { Ladder, } 10 \text { to } 250 \mathrm{kDa}\end{array}$ & 26619 & $\begin{array}{l}\text { Thermo Fisher Scientific, } \\
\text { Waltham, MA, USA }\end{array}$ \\
\hline Phosphatase Inhibitor Cocktail 1 & P2850-5ml & $\begin{array}{l}\text { Sigma-Aldrich, Munich, } \\
\text { Germany }\end{array}$ \\
\hline Phosphatase Inhibitor Cocktail 2 & P5726-5ml & $\begin{array}{l}\text { Sigma-Aldrich, Munich, } \\
\text { Germany }\end{array}$ \\
\hline Proteinase $\mathrm{K}$ & 000000003115836001 & $\begin{array}{l}\text { Sigma-Aldrich, Munich, } \\
\text { Germany }\end{array}$ \\
\hline Tamoxifen & Cas\#10540-29-1 & $\begin{array}{l}\text { Sigma-Aldrich, Munich, } \\
\text { Germany }\end{array}$ \\
\hline Tissuetec $\mathbb{B}$ & 4583 & $\begin{array}{l}\text { Jung HistoService, Nussloch, } \\
\text { Germany }\end{array}$ \\
\hline Whatman $\AA$ Protran Nitrocellulose & Z613630 & Whatman GmbH, Dassel, \\
\hline
\end{tabular}




\subsubsection{Primers}

All primers used in this thesis are listed below in Table 6. Primer stocks were ordered in $50 \mathrm{mM}$ concentration either from the local AGCT core facility (Max-Planck Institute of Experimental Medicine, Göttingen, Germany) or from Sigma Aldrich. Both primer stocks and $10 \mathrm{mM}$ working aliquots were stored at $-20^{\circ} \mathrm{C}$.

Table 6: List of primers.

\begin{tabular}{|c|c|c|}
\hline $\begin{array}{l}\text { Primer } \\
\text { Name } \\
\end{array}$ & Description & Primer sequence [5' -3'] \\
\hline 24050 & Fwd for $\mathrm{CX}_{3} \mathrm{CR} 1-\mathrm{Cre}^{\mathrm{ERT} 2}$ & TATCTTCTATATCTTCAGGCGC \\
\hline 24051 & Rev for $\mathrm{CX}_{3} \mathrm{CR} 1-\mathrm{Cre} \mathrm{ERT2}^{\mathrm{ER}}$ & GTGAACGAACCTGGTCGAAATCAG \\
\hline 36122 & Fwd for $\mathrm{CX}_{3} \mathrm{CR} 1-\mathrm{Cre}^{+/+}$ & AGCTCACGACTGCCTTCTT \\
\hline 36123 & Rev for $\mathrm{CX}_{3} \mathrm{CR} 1-\mathrm{Cre}^{+/+}$ & ACGCCCAGACTAATGGTGAC \\
\hline 24052 & Fwd for Rab7 floxed site & CTCACTCACTCCTAAATGG \\
\hline 24053 & Rev for Rab7 floxed site & TTAGGCTGTATGTATGTGC \\
\hline 18718 & Fwd for PS1 mutations in 5xFAD model & AATAGAGAACGGCAGGAGCA \\
\hline 18719 & Rev for PS1 mutations in 5xFAD model & GCCATGAGGGCACTAATCAT \\
\hline 33022 & Fwd for APP mutations in 5xFAD model & AGGACTGACCACTCGACCAG \\
\hline 33023 & Rev for APP mutations in 5xFAD model & CGGGGGTCTAGTTCTGCAT \\
\hline
\end{tabular}

\subsubsection{Antibodies}

Primary antibodies used in this project, their application and the correspondent concentrations are listed in Table 7. A summary of all secondary antibodies with their respective conjugates that were used in this thesis are shown in Table 8.

Table 7: Primary antibodies.

\begin{tabular}{lllll}
\hline \hline Antibody & Host species & Company & $\begin{array}{l}\text { Catalog } \\
\text { number }\end{array}$ & $\begin{array}{l}\text { Working } \\
\text { dilution } \\
\text { (Application) }\end{array}$ \\
\hline \hline 6E10 & mouse & Covance & SIG-39300 & $1: 2000(\mathrm{IHC})$ \\
\hline A 342 & rabbit & $\begin{array}{l}\text { Invitrogen/Thermo } \\
\text { Fisher }\end{array}$ & $44-344$ & $1: 1000(\mathrm{IHC})$ \\
\hline Calnexin & rabbit & Sigma Aldrich & C4731 & $1: 1000(\mathrm{WB})$ \\
\hline CD68 & rat & BD /Thermo Fisher & NC 9471873 & $1: 500(\mathrm{HC})$ \\
\hline GFAP & ginea pig & Synaptic Systems & 173004 & $1: 500(\mathrm{HC})$ \\
\hline Iba1 & rabbit & WAKO & $019-19741 /$ & $1: 2000(\mathrm{IHC})$ \\
\hline Iba1 & goat & Abcam & NC9288364 & \\
\hline LAMP1 (CD107a) & rat & BD & ab5076 & $1: 1000(\mathrm{IHC})$ \\
\hline NeuN & mouse & Millipore & MAB3792 & $1: 500(\mathrm{IHC})$ \\
\hline Synaptophysin & rabbit & Abcam & ab16659 & $1: 500(\mathrm{IHC})$ \\
\hline \hline
\end{tabular}


Table 8: Secondary antibodies.

\begin{tabular}{llllll}
\hline \hline Antibody & Host & Conjugate & Company & $\begin{array}{l}\text { catalog } \\
\text { number }\end{array}$ & $\begin{array}{l}\text { Working } \\
\text { dilution } \\
\text { (Application) }\end{array}$ \\
\hline \hline Anti-ginea pig & goat & Alexa555 & Invitrogen & 1900250 & $1: 300(\mathrm{IHC})$ \\
\hline Anti-goat & donkey & Alexa555 & Thermo Fisher & A21432 & $1: 300(\mathrm{IHC})$ \\
\hline Anti-mouse & rabbit & Alexa488 & Invitrogen & A11008 & $1: 300(\mathrm{IHC})$ \\
\hline Anti-mouse & goat & Cy3 & Dianova & $115-165-146$ & $1: 300(\mathrm{IHC})$ \\
\hline Anti-mouse & donkey & Alexa647 & Thermo Fisher & A31571 & $1: 300(\mathrm{IHC})$ \\
\hline Anti-mouse & goat & HRP & Dianova & $115-035-062$ & $1: 2000(\mathrm{WB})$ \\
\hline Anti-rabbit & goat & Alexa488 & Invitrogen & A11008 & $1: 300(\mathrm{IHC})$ \\
\hline Anti-rabbit & goat & Cy3 & Dianova & $111-165-144$ & $1: 300(\mathrm{IHC})$ \\
\hline Anti-rabbit & donkey & Alexa647 & Invitrogen & 1964354 & $1: 300(\mathrm{IHC})$ \\
\hline Anti-rabbit & goat & HRP & Dianova & $111-035-003$ & $1: 2000(\mathrm{WB})$ \\
\hline Anti-rat & donkey & Alexa488 & Thermo Fisher & A21208 & $1: 300(\mathrm{IHC})$ \\
\hline Anti-rat & goat & Cy3 & Invitrogen & 1842804 & $1: 300(\mathrm{IHC})$ \\
\hline \hline
\end{tabular}

\subsubsection{Equipment}

Table 9: List of equipment.

\begin{tabular}{|c|c|}
\hline Equipment & Company \\
\hline Precellys 24-Dual Tissue homogenizer & $\begin{array}{l}\text { Bertin intstruments, Montigny-le-Bretonneux, } \\
\text { France }\end{array}$ \\
\hline Bio-Rad- Mini-Sub ${ }^{\circledR}$ Cell GT Systems & Bio-Rad Laboratories GmbH, Munich, Germany \\
\hline $\begin{array}{l}\text { Bio-Rad Mini-PROTEAN® Tetra electrophoresis } \\
\text { system }\end{array}$ & Bio-Rad Laboratories GmbH, Munich, Germany \\
\hline Cryostate 3050 S & Leica Microsystems, Nussloch, Germany \\
\hline Mini-Trans Blot cell set up & Bio-Rad Laboratories GmbH, Munich, Germany \\
\hline PELCO Prep-Eze ${ }^{\text {TM }}$ 24-wellplate Insert & PELCO, Redding, CA, USA \\
\hline FLUOstar omega plate reader & BMG Labtech, Ortenberg, Germany \\
\hline Rotor TLA-55 & Beckman Counter, Indianapolis, IN USA \\
\hline Sector Imager 6000 & Meso Scale Discovery ${ }^{\mathrm{TM}}$, Rockville, MD, USA \\
\hline Sonicator CL-18 & Thermo Fisher Scientific Inc,Rockford, IL, USA \\
\hline Heraeus $^{\mathrm{TM}}$ Fresco $^{\mathrm{TM}} 17$, microcentrifuge & Thermo Fisher Scientific Inc,Rockford, IL, USA \\
\hline Thermomixer comfort & Eppendorf, Hamburg, Germany \\
\hline T3000 Thermocycler Kombi & Biometra, Göttingen, Germany \\
\hline Ultracentrifuge Optima Max-XP & Beckman Counter, Indianapolis, IN USA \\
\hline Vibratome VT1200S & Leica Microsystems, Nussloch, Germany \\
\hline
\end{tabular}

\subsubsection{Software}

Table 10: List of software.

\begin{tabular}{lll}
\hline \hline Software & Source & Application \\
\hline \hline Adobe Illustrator CS5.1 & Adobe Systems Inc & Illustration of figures \\
\hline EndNote X8 & Clarivate Analytics & Bibliography \\
\hline GraphPad Prism® 6 & GraphPad Software Inc. & $\begin{array}{l}\text { Statistical analyses and } \\
\text { graph generation }\end{array}$ \\
\hline Image J & & Image processing and \\
\hline
\end{tabular}




\begin{tabular}{lll}
\hline & & analysis \\
\hline Imaris 6 & Bitplane, Oxford Instruments & $\begin{array}{l}\text { 3D image analysis ; e.g. } \\
\text { NeuN quantification }\end{array}$ \\
\hline MS Office Excel 2010 & Microsoft & Data analysis \\
\hline MS Office Word 2010 & Microsoft & Text writing and editing \\
\hline MSD Discovery Workbench & Meso Scale Discovery ${ }^{\text {TM }}$, & $\begin{array}{l}\text { Measurement and analysis } \\
\text { of MSD assay }\end{array}$ \\
\hline 3.0 Data Analysis Toolbox & Rockville, MD, USA & Measurement of BCA assay \\
\hline Omega Control & BMG Labtech, Ortenberg, & Germany \\
\hline Reconstruct & John C. Fiala & $\begin{array}{l}\text { Dendritic spine analysis of } \\
\text { Golgi-Cox staining }\end{array}$ \\
\hline Zen blue 2.3 lite & Zeiss, Oberkochen, Germany & $\begin{array}{l}\text { Image processing and } \\
\text { analysis }\end{array}$ \\
\hline \hline
\end{tabular}

\subsection{Methods}

\subsubsection{Generation of the $R a b 7^{\Delta M G} \times 5 x F A D$ mouse line}

In this project I have analyzed a triple transgenic mouse model generated from the cross-breeding of three established mouse models: the $\mathrm{CX}_{3} \mathrm{CR} 1-\mathrm{Cre} \mathrm{ERT}^{\mathrm{ER} /+}$ mouse line, the Rab7//fl/ mouse line and the $5 x F A D$ mouse line (see Figure 12). The name of the model is abbreviated as the Rab7 ${ }^{\triangle M G} x$ 5xFAD.

To achieve a disruption within the endo-lysosomal pathway in microglia, the monocyte-specific, Tamoxifen-inducible $\mathrm{CX}_{3} \mathrm{CR} 1-\mathrm{Cr} \mathrm{E}^{\mathrm{ERT} 2 /+}$ mouse (originally from Dr. Steffen Jung, Department of Immunology, Weizmann Institute of Science, purchased via Jackson laboratories: 020940) was used in combination with a mouse line having loxP site flanked exon 2 of the Rab7 (Rab7 $\left.{ }^{f / f f}\right)$ alleles (originally from Dr. Aimee Edinger, Department of Cell \& Developmental Biology University of California, Irvine). This $\mathrm{CX}_{3} \mathrm{CR} 1-\mathrm{Cr} \mathrm{e}^{\mathrm{ERT} 2 /+}$-mediated Rab7 knockout (KO; Rab7 ${ }^{\Delta \mathrm{MG}}$ ) mouse line was previously established and characterized by Shima Safaiyan in terms of myelin turnover (Safaiyan et al., 2016).

To investigate the impact of the endo-lysosomal pathway in microglia on the progression of $A D$, the $\mathrm{CX}_{3} \mathrm{CR} 1-\mathrm{Cre} \mathrm{ERT2/+}^{\mathrm{E}} \times \mathrm{Rab7}^{\mathrm{fl/fl}}$ mouse line was crossed with the 5xFAD mouse model (Oakley et al., 2006). The 5x FAD model heterozygous (het) expresses the human isoform of APP 695 bearing the Swedish, London and Florida mutations and the human PSEN1 bearing the M146L and the L286V mutations with the neuronal Thy1-promoter, respectively (see Figure 12). Therefore the animals develop early $A \beta$-pathology.

For this study animals with the following genotype were used: $\mathrm{CX}_{3} \mathrm{CR} 1-\mathrm{Cre}^{\mathrm{ERT} 2 /+} \mathrm{Rab} 7^{\mathrm{fl} / \mathrm{fl}} 5 \mathrm{xFAD} \mathrm{D}^{\text {het }}$. Even though most studies in $A D$ mice focus on female mice, due to their stronger phenotype, we have also investigated male mice. 

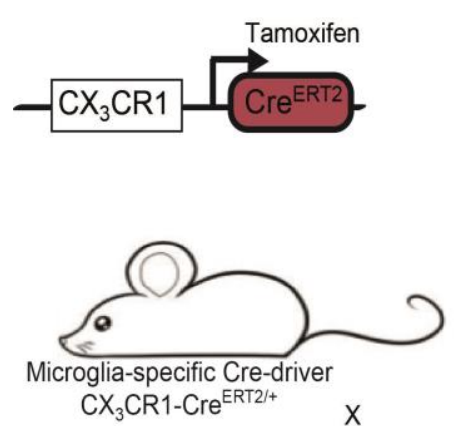
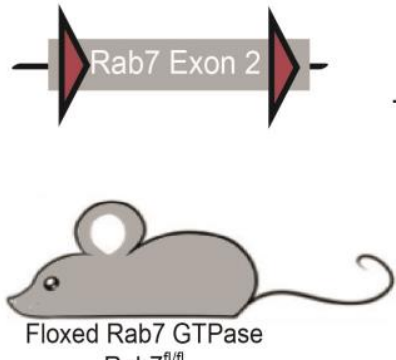

$\operatorname{Rab} 7^{\text {ff/fl }}$

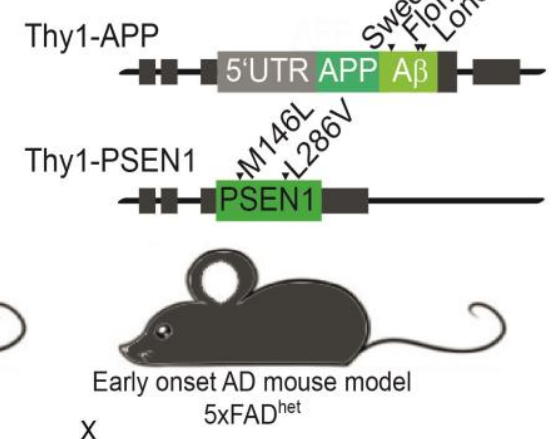

Figure 12: Generation of the Rab7 ${ }^{\triangle M G} \times 5 x F A D$ mouse line.

The mouse line was generated by cross-breeding a tamoxifen-inducible Cre-driver line under transcriptional control of the myeloid cell specific $\mathrm{CX}_{3} \mathrm{CR} 1$ promoter (left) with the Rab7 $7^{\mathrm{flfl} l}$ line (middle), bearing two lox p sites (fl; red) flanking exon 2 of the Rab7 allele. Thus, only after application of tamoxifen and thereby induction of Cre expression, the Rab7 flanking lox $\mathrm{p}$ sites were cleaved resulting in a conditional Rab7 KO in $\mathrm{CX}_{3} \mathrm{CR} 1+$ myeloid cells (Rab7 ${ }^{\Delta \mathrm{MG}}$ ). These mice were further crossed with the early onset $A D$ mouse model 5xFAD. The 5xFAD mouse model overexpresses human $A P P$ and human PSEN1 (both in green) under the transcriptional control of the neuronal Thy1-promoter. APP and PSEN bear mutations found in familial cases of $A D$, respectively. Black triangles mark the mutation sites. Little grey boxes indicate Thy1 exons.

\subsubsection{Tamoxifen-induced depletion of Rab7}

To induce the $\mathrm{CX}_{3} \mathrm{CR}$-Cre $\mathrm{ERT}^{\mathrm{ER} /+}$-mediated knockout of the Rab7 f//fl alleles, tamoxifen was administered intraperitoneal (i.p.) to P21 mice once every 24 hours, for 7 consecutive days. For the tamoxifen treatment, $10 \mathrm{mg} / \mathrm{ml}$ solutions of tamoxifen in sterile-filtered corn oil were prepared freshly the day before injection. The solution was vortexed and shook overnight (ON) at $37^{\circ} \mathrm{C}$ with maximum speed until the suspension was homogenous. Throughout the whole preparation the solution was light-protected. Animals were injected with $75 \mathrm{mg}$ tamoxifen/ $\mathrm{kg}$ body weight using $1 \mathrm{ml}$ syringes and 3/8" beveled needle - 26 gauge.

The first aging cohort was sacrificed at 3 months of age when 5 XFAD mice were reported to have already developed $A \beta$-plaques in the hippocampus and in the cortex in both sexes (Oakley et al., 2006). The second aging cohort was dissected at 9 months of age when neuronal loss was detectable in 5xFAD mice.

\subsubsection{Genotyping}

To discriminate animals with the proper genotype for the experiments $-\mathrm{Cre}^{\mathrm{ERT} 2 /+} \mathrm{Rab} 7^{\mathrm{fl} / \mathrm{fl}} 5 \times \mathrm{FAD} \mathrm{D}^{\text {het }}-$ from littermates with different genotypes, genomic DNA isolation and PCR amplification was done according to the following protocols. 


\subsubsection{DNA isolation}

DNA was isolated either from small tail tips of P1-P5 animals or from ear punches of P14-P18 animals. The tissue was digested $\mathrm{ON}$ at $55^{\circ} \mathrm{C}$ in a thermomixer with $350 \mu$ tail lysis buffer and $20 \mu \mathrm{l}$ Proteinase $\mathrm{K}(10 \mathrm{mg} / \mathrm{ml}$ stock) respectively. The supernatant was collected after centrifuging for $5 \mathrm{~min}$ at RT at 13,200 $\mathrm{g}$. To precipitate the DNA $350 \mu \mathrm{l}$ isopropanol was added and samples were repeatedly centrifuged and the supernatant discard. The DNA pellet was washed with $70 \%$ [v/v]

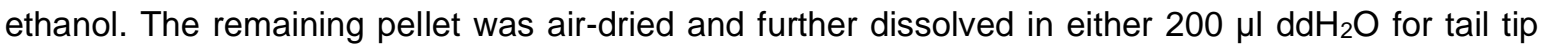
obtained DNA or in $100 \mu \mathrm{lddH_{2 }} O$ for ear puncher obtained DNA. The DNA samples were stored at $4^{\circ} \mathrm{C}$. The isolated DNA was used for genotyping using polymerase chain reaction (PCR).

\subsubsection{Polymerase Chain Reaction (PCR)}

To determine the genotypes of the mice from the Rab7 ${ }^{\triangle M G} \times 5 \times F A D$ mouse line, the isolated, genomic DNA was specifically amplified by polymerase chain reaction (PCR). The compounds for the PCR reactions were purchased from Promega. Primer sequences are listed in Table 6.

PCR products were size-separated on $1 \%[\mathrm{w} / \mathrm{v}]$ agarose gels casted with $1 \mathrm{x}$ TBE and containing $3.5 \%[\mathrm{v} / \mathrm{v}]$ gel red. For size reference $5 \mu \mathrm{l}$ 100bp "plus" DNA ladder was used. Depending on the band sizes for the PCRs, the runs were between $30 \mathrm{~min}$ to $90 \mathrm{~min}$ at $140 \mathrm{~V}$.

The following PCR-protocols were used to genotype the animals:

Table 11: PCR for $\mathrm{CX}_{3} \mathrm{CR}-\mathrm{Cre}^{+/+}$wildtype allele.

\begin{tabular}{ll}
\hline \hline Reagent & Volume $[\mu l]$ \\
\hline \hline BQ 5x & 5.0 \\
\hline dNTPs & 0.5 \\
\hline $\mathrm{MgCl}_{2}$ & 1.0 \\
\hline Primer 36122[10pmol] & 1.0 \\
\hline Primer 36123 [10pmol] & 1.0 \\
\hline GoTaq pol & 0.1 \\
\hline $\mathrm{H}_{2} 0$ & 14.4 \\
\hline DNA & 2.0 \\
\hline \hline
\end{tabular}

Table 12: $\mathrm{CX}_{3} \mathrm{CR}_{\mathrm{C}} \mathrm{Cre}^{\mathrm{ERT} 2 /+} \mathrm{PCR}$ protocol.

\begin{tabular}{|c|c|}
\hline Reagent & Volume [ $\mu \mathrm{l}]$ \\
\hline$B Q 5 x$ & 5.0 \\
\hline dNTPs & 0.5 \\
\hline $\mathrm{MgCl}_{2}$ & 1.0 \\
\hline Primer 24050 [10pmol] & 1.0 \\
\hline Primer 24051 [10pmol] & 1.0 \\
\hline GoTaq pol & 0.1 \\
\hline $\mathrm{H}_{2} \mathrm{O}$ & 14.4 \\
\hline DNA & 2.0 \\
\hline
\end{tabular}


Table 13: PCR program for $\mathrm{CX}_{3} \mathrm{CR}_{-\mathrm{Cre}}{ }^{+/+}$wildtype and $\mathrm{CX}_{3} \mathrm{CR}-\mathrm{Cre} \mathrm{ERT2/+}^{\mathrm{PCR}}$.

\begin{tabular}{|c|c|c|}
\hline Temperature $\left[{ }^{\circ} \mathrm{C}\right]$ & Duration & Cycles \\
\hline 94 & $3 \min$ & $1 \mathrm{x}$ \\
\hline 94 & $45 \mathrm{sec}$ & \multirow{3}{*}{$35 x$} \\
\hline 58 & $45 \mathrm{sec}$ & \\
\hline 72 & $1 \mathrm{~min}$ & \\
\hline 72 & $5 \min$ & $1 \mathrm{x}$ \\
\hline 4 & hold & \\
\hline
\end{tabular}

The Cre-insertion $\left(\mathrm{CX}_{3} \mathrm{CR}-\mathrm{Cre}^{\mathrm{ERT} 2 /+}\right)$ resulted in a 223 base pairs (bp) product whereas no insertion of the Cre $\left(\mathrm{CX}_{3} \mathrm{CR}-\mathrm{Cre}{ }^{++}\right)$amplified a $151 \mathrm{bp}$ PCR product.

Table 14: PCR reaction for Rab7 $7^{\text {fl/fl }}$.

\begin{tabular}{ll}
\hline \hline Reagent & Volume $[\mu \mathrm{ll}]$ \\
\hline \hline BQ 5x & 5.0 \\
\hline dNTPs & 0.5 \\
\hline $\mathrm{MgCl}_{2}$ & 1.0 \\
\hline Primer 24052 [10pmol] & 1.0 \\
\hline Primer 24053 [10pmol] & 1.0 \\
\hline GoTaq pol & 0.1 \\
\hline $\mathrm{H}_{2} 0$ & 15.4 \\
\hline DNA & 1.0 \\
\hline \hline
\end{tabular}

Table 15: PCR program for Rab7 $7^{\mathrm{fl/fl}}$ PCR.

\begin{tabular}{|c|c|c|}
\hline Temperature $\left[{ }^{\circ} \mathbf{C}\right]$ & Duration [min] & Cycles \\
\hline 94 & 5 & $1 \mathrm{x}$ \\
\hline 94 & 1 & \multirow{3}{*}{$35 x$} \\
\hline 55 & 1 & \\
\hline 72 & 2 & \\
\hline 72 & 10 & $1 \mathrm{x}$ \\
\hline 4 & hold & \\
\hline
\end{tabular}

Insertion of lox-sited in the Rab7 allele resulted in a 580 bp band whereas Rab7 wildtype was shown in a 550 bp product.

Table 16: PCR protocol for 5xFAD PCR.

\begin{tabular}{ll}
\hline \hline Reagent & Volume $[\mu l]$ \\
\hline \hline BQ 5x & 5.0 \\
\hline dNTPs & 0.5 \\
\hline $\mathrm{MgCl}_{2}$ & 1.0 \\
\hline Primer 18718 (PSI fw) [10pmol] & 1.0 \\
\hline Primer 18719 (PSI rev) [10pmol] & 1.0 \\
\hline Primer 33022 (APP fw) [10pmol] & 1.0 \\
\hline Primer 33023 (APP rev) [10pmol] & 1.0 \\
\hline GoTaq pol & 0.15 \\
\hline $\mathrm{H}_{2} 0$ & 12.85 \\
\hline DNA & 2.0 \\
\hline \hline
\end{tabular}


Table 17: PCR program for 5xFAD PCR.

\begin{tabular}{lll}
\hline \hline Temperature $\left[{ }^{\circ} \mathrm{C}\right]$ & Duration & Cycles \\
\hline \hline 94 & $3 \mathrm{~min}$ & $1 \mathrm{x}$ \\
\hline 94 & $45 \mathrm{sec}$ & $35 \mathrm{x}$ \\
\hline 63 & $45 \mathrm{sec}$ & \\
\hline 72 & $1 \mathrm{~min}$ & $1 \mathrm{x}$ \\
\hline 72 & $3 \mathrm{~min}$ & \\
\hline 4 & hold & \\
\hline \hline
\end{tabular}

Mutations within the APP resulted in a PCR product of $377 \mathrm{bp}$. The PS1 mutations were verified by a 608 bp band.

\subsubsection{Histology and tissue preparation 2.2.4.1.Perfusion and plasma isolation}

Mice were anesthetized for surgery by i.p. injection of $20 \mu \mathrm{l} / \mathrm{g}$ body weight Avertin stock solution. Blood was isolated by cardiac puncture with $15 \mu \mathrm{l} 250 \mathrm{mM}$ EDTA-coated $1 \mathrm{ml}$ syringes with a 25xG needle. For plasma isolation the blood was processed within 20 min after drawing and centrifuged for $10 \mathrm{~min}$ at RT and $1.300 \times \mathrm{g}$. The plasma was transferred into a fresh tubed, snap-frozen and stored at $-80^{\circ} \mathrm{C}$ until further use.

Afterwards, mice were perfused with ice-cold PBS for $15 \mathrm{~min}$. The brain was dissected. The left hemibrain was post-fixed for $48 \mathrm{~h}$ in $4 \%$ PFA at $4^{\circ} \mathrm{C}$ for immunohistochemistry (IHC). Then the tissue was de-hydrated for at least $48 \mathrm{~h}$ with $30 \%$ [w/v] sucrose in PBS, before it was embedded in Tissuetec $\circledast$ and stored at $-80^{\circ} \mathrm{C}$ until cryosectioning (see section 2.2.4.2).

From the right hemibrain the hippocampus (HPC) as a whole were dissected and snap-frozen. These samples were used for biochemical analyses.

\subsubsection{Cryosectioning}

Coronal sections of $30 \mu \mathrm{m}$ were sliced with a cryostate. Every $12^{\text {th }}$ section was collected with a brush and placed in consecutive order in a 12-well plate. The start point of collection was the prefrontal cortex and the last sections collected were from the mid-part of the cerebellum. The sections were stored in cryoprotective solution at $4^{\circ} \mathrm{C}$ until usage. To avoid evaporation, the plates were sealed with parafilm.

\subsubsection{Free-floating immunohistochemistry with fluorescence detection}

All free-floating stainings were done in 12-, 24- or 48-well plates, depending on the number of sections picked per animal. For stainings of whole series ergo, every $12^{\text {th }}$ section netwells were used for all steps, except for the blocking and antibody incubations. Sections were briefly washed 
with $0.1 \%$ TBST. For permeabilization sections were incubated with $0.1 \%$ Triton- $\mathrm{X}, 0.6 \% \mathrm{H}_{2} \mathrm{O}_{2}$ in $0.1 \%$ TBST for 30 min at RT. Sections were washed 3 times for 5 min and blocked with $10 \%$ normal donkey serum in $0.1 \%$ TBST for $1 \mathrm{~h}$ at RT. Primary antibodies were diluted in $2 \%$ normal donkey serum in $0.1 \%$ TBST and incubated at $4^{\circ} \mathrm{C} \mathrm{ON}$. A list of used primary antibodies and their working dilutions is shown in Table 7. Fluorophore-coupled species-specific secondary antibodies were all used in 1:300 dilution in 2\% normal donkey serum in 0.1\% TBS and were incubated for $1-2 \mathrm{~h}$ at RT protected from light. Secondary antibodies used in this project are listed in Table 8. Sections were washed 3 times for 5 min with $0.1 \%$ TBST. Then the sections were placed carefully with a brush on HistoBond-adhesion-micro-glass slides and dried at RT in the dark. For mounting Mowiol supplemented with $0.1 \%$ [w/v] DAPI (4', 6-Diamidino-2'-Phenylindol) was used together with cover slips no 1 . Slides were dried $\mathrm{ON}$ at RT in the dark and stored light-protected at $4^{\circ} \mathrm{C}$.

\section{Antigen retrieval:}

Some antibodies require antigen retrieval since tissue sections were fixed with PFA. If needed, this step was added after the permeabilization and washing.

In brief, sections were treated for $15 \mathrm{~min}$ with pre-warmed ciric buffer at $80^{\circ} \mathrm{C}$ in a water bath. Afterwards the samples were cooled down on ice to RT.

For 6E10 staining antigen retrieval with formic acid was done, for a better antibody binding. Therefore, the sections were incubated with $70 \%$ [v/v] formic acid in $\mathrm{H}_{2} \mathrm{O}$ for 15 min at RT.

Afterwards sections were washed 3 times for 5 min with $0.1 \%$ TBST, before continuing with the blocking step.

\section{Two-step staining:}

Some antibodies were not compatible with the conditions, e.g. antigen retrieval, of the antibodies used in a co-staining approach. Then the staining was split into a two-step protocol.

The sensitive antibodies, which did not appreciate antigen retrieval, were used according to the previous described staining protocol. After this first staining was completed, the bound antibodies were fixed by incubating the sections for $40 \mathrm{~min}$ at RT in $4 \%$ PFA. Sections were washed properly for 3 times for $10 \mathrm{~min}$ with $0.1 \%$ TBST. Then the staining protocol including the antigen retrieval step was done with these sections and the remaining antibody. After the second staining was completed, sections were mounted and cover slipped as described before.

This protocol was e.g. used for the CD68, Iba1 staining with an additional $6 \mathrm{E} 10$ staining using $70 \%$ formic acid antigen retrieval.

\section{Sudan Black treatment:}

To reduce high autofluorescence from lipofuscin in aged tissue, sections were treated with sudan black after the fluorescent staining was completed. Sections were incubated for $15 \mathrm{~min}$ in 
$0.02 \%[\mathrm{w} / \mathrm{v}]$ sudan black in $70 \%[\mathrm{v} / \mathrm{v}]$ ethanol in $\mathrm{ddH}_{2} \mathrm{O}$ at $\mathrm{RT}$ on the shaker. Sections were washed three times for 5 min with $0.1 \%$ TBST and mounted as described.

\subsubsection{Thioflavin S staining}

Thioflavin S (ThioS) binds to $\beta$-sheet rich structures, resulting in specific labeling of the dense core of $A \beta$-plaques. The extinction peaks is at $342 \mathrm{~nm}$ and the emission maximum is around $440 \mathrm{~nm}$. Hence, Thios signal is detected in the green channel and spreads into the blue one as well, prohibiting additional DAPI staining.

For additional ThioS staining sections were incubated for $15 \mathrm{~min}$ in $0.01 \%$ [w/v] ThioS in TBS in the dark, after secondary antibody treatment. Then sections were rinsed 2 times in $50 \%$ [v/v] ethanol and washed 2 times for $10 \mathrm{~min}$ in $1 \mathrm{x}$ TBS, before mounted on HistoBond-adhesion-micro-glass slides and sealed with Mowiol without DAPI supplement.

\subsubsection{Golgi-Cox staining}

To analyze dendritic spine loss, Golgi-Cox staining was done with brain tissue of 9 month old animals. For the Golgi-Cox staining the protocol published by Zaqout and Kaindl (2016) was used. The composition of the used buffers and solutions is described in section 2.1.1.

Briefly, one freshly isolated, PBS-perfused hemibrain was placed into $10 \mathrm{ml}$ freshly prepared GolgiCox solution ON at RT in the dark. The solution was exchanged after $24 \mathrm{~h}$. After 7-9 days the brains were carefully transferred into a conical with Tissue Protectant Solution and stored at $4^{\circ} \mathrm{C}$ in the dark. This solution was refreshed after $24 \mathrm{~h}$ and the brains were further de-hydrated for 7 more days. The impregnated tissue was embedded in $3 \%[\mathrm{w} / \mathrm{v}]$ agarose/PBS and sliced with the vibratome VT1200S sections using $60 \mathrm{~Hz}$ and $15 \mathrm{~mm} / \mathrm{s}$ into $100 \mu \mathrm{m}$ thick sections. The vibratome chamber was filled with cooled Golgi-Cox Tissue Protectant Solution which was prior filtered

through a $240 \mathrm{~nm}$ filter paper. Every forth section was collected on a pork gelatin-coated slide. The preparation of the gelatin-coated slides is described in detail at the end of the section. When all sections were collected, they were gently pushed into the gelatin matrix.

The vibratome sections were dried at RT for $48 \mathrm{~h}$ in a dust-free environment.

Afterwards the Golgi-Cox-staining was developed inside a fume hood according to the following protocol:

- $2 \times 5$ min $\mathrm{ddH}_{2} \mathrm{O}$

- $5 \min 50 \%[v / v]$ ethanol

- 8 min 3:1 ammonia

- $2 \times 5$ min $\mathrm{ddH}_{2} \mathrm{O}$

- $10 \min 5 \%[\mathrm{w} / \mathrm{v}]$ sodium thiosulfate (this step was done in the dark)

- $2 x 1$ min $d d H_{2} \mathrm{O}$

- $6 \min 70 \%[v / v]$ ethanol 
- $6 \min 95 \%[\mathrm{v} / \mathrm{v}]$ ethanol

- $6 \min 100 \%[v / v]$ ethanol

- At least 6 min xylol (until mounting step)

Samples were mounted with 10 drops of Eukitt hard-drying medium and evaporated in the fume hood for at least $48 \mathrm{~h}$ before imaging.

\section{Gelatin-coated slides:}

To obtain gelatin-coated slides, common glass microscopy slides were placed in a metal histology staining rack and rinsed briefly with $\mathrm{ddH}_{2} \mathrm{O}$. Slides were allowed to dry in a dust-free area for at least $2 \mathrm{~h}$. Meanwhile $3 \%[\mathrm{w} / \mathrm{v}]$ commonly available pork gelatin in $\mathrm{dd}_{2} \mathrm{O}$ was dissolved by heating to $55^{\circ} \mathrm{C}$ with constant stirring. The gelatin was filtered through a $240 \mathrm{~nm}$ filter paper into a clean histological staining box. The dried glass slides were immersed for $10 \mathrm{~min}$. Then the rack was removed and the slides were allowed to dry ON at RT in a dust-free environment. Gelatin-coated slides were stored for up to one month at $4^{\circ} \mathrm{C}$ in a closed histological cassette.

\subsubsection{Imaging}

For imaging microscopes within the Light Microscopy Facility of the DZNE in Bonn were used. Samples were blinded before imaging and unblinded after analysis of the respective images. To image with high magnification, 40x, 63x and 100x oil objectives were used in combination with Immersol $518 \mathrm{~F}$.

All objectives used in this study are listed in the table below:

Table 18: Microscope objectives used in this study.

\begin{tabular}{lll}
\hline \hline Microscope & Magnification & Objective \\
\hline \hline AxioScan Z1 & $5 \mathrm{x}$ & Plan-Apochromat, 0.25, M27 Air \\
\hline Confocal LSM700 and AxioScan Z1 & $20 \mathrm{x}$ & Plan-Apochromat, 0.8, M27 Air \\
\hline Confocal LSM700 & $40 \mathrm{x}$ & Plan-Apochromat, 1.3 Oil, DIC III \\
\hline Confocal LSM700 & $63 \mathrm{x}$ & Plan-Apochromat, 1.4 Oil DIC M27 \\
\hline Epi-Scope & $100 \mathrm{x}$ & Plan-Apochromat 1.40 Oil Ph 3 M27 \\
\hline \hline
\end{tabular}

\subsubsection{LSM700 Confocal}

Confocal imaging was used for higher resolution, to visualize spatial plaque morphology, investigate subcellular structures such as lysosomes and to allow for 3 dimensional reconstruction amongst others for cellular morphology analysis.

To analyze neuronal loss, the NeuN staining was imaged with $20 x$ for further three dimensional cell counting using Imaris software. Therefore $z$-stacks with step sizes of $1.5 \mu \mathrm{m}$ and tile scans with $2 \%$ overlap were taken. Images with 8-bit depth were taken, using bi-directional, non-averaged line scanning. Image resolution was 512 x 512 pixels. Per animal the cortex was imaged in four slices 
and the subiculum was imaged in two sections each. The sections had $360 \mu \mathrm{m}$ distance from one another.

For the analysis of spatial $A \beta$ plaques architecture, triple plaque stainings using the markers ThioS, $A \beta_{42}$ and $6 \mathrm{E} 10$ were imaged within cortical layer $\mathrm{V}$, dorsal to the CA1 region at a section using the $63 x$ objective. The whole thickness of the section was scanned with a step size of $0.75 \mu \mathrm{m}$. Tile scans with $2.5 \%$ overlap were imaged, capturing at least 6 plaques per animal. Images were taken with 16-bit and $512 \times 512$ pixel resolution.

The subcellular stainings with either LAMP1 together with $A \beta_{42}$ and Iba1 or GFAP were imaged with the 40x oil objective. For the analyses of fluorescence intensity within Iba1+ or GFAP+ cells, tilescans within a single $z$-plane were taken with $5 \%$ overlap. At least $7 \times 7$ tiles, with 16 -bit and pixels $1024 \times 1024$ pixel were imaged within the cortex.

For the analysis of microglia morphology, LAMP1+ lysosome volumes and CD68 volumes, z-stacks throughout the whole thickness of the section were taken within the cortex, dorsal to the CA1 region. Images with z-stacks with $1 \mu \mathrm{m}$, resolution of 16-bit and $1024 \times 1024$ pixel were taken using the $40 x$ oil objective. These images were processed using Imaris software.

\subsubsection{AxioScan Z1}

For quantification of lba1+ microglia, GFAP+ astrocyte and 6E10+ plaque numbers, whole sections were semi-automatically imaged using the Axioscan Z1 microscope (Zeiss, Oberkochen, Germany). All sections including a part of the hippocampus were manually marked as region of interest and were chosen for imaging. Tissue detection was achieved with transmitted light at $5 x$ magnification. To find the focus within the specimen DAPI signal was chosen for the GFAP, Iba1 and 6E10 staining. Using the coarse focus point found within the reference channel, a range of $60 \mu \mathrm{m}$ was scanned within every individual tile for fine focusing. Fluorescence images were obtained using a 20x objective. Images were taken with 16-bit depth and 1024x1024 pixels. Tile scans were stitched automatically due to the selected reference channel. Every brain section selected was saved as a scene within a glass slide / animal dependent file. Identical stainings from both aging cohorts were imaged with the same settings. Images were saved as czi-files.

\subsubsection{Epi-Scope}

The Epi-Scope (Zeiss, Oberkochen, Germany) was used to image the spines, stained by Golgi-Cox method (2.2.4.5). Therefore a $100 \mathrm{x}$ oil objective was used. Secondary dendrites within the $5^{\text {th }}$ cortical layer were selected for focusing. Images were taken with 12-bit and $0.5 \mu \mathrm{m} z$-steps. Per animal at least 15 dendrites were imaged and further analyzed as described in detail in (Zaqout and Kaindl, 2016). 


\subsubsection{Image processing and data analysis 2.2.6.1.Fiji (Fiji is just ImageJ)}

For image analysis slide scanner images were opened in ZEN blue software to add scale bare to the individual scenes which equals brain sections. Then images were exported as JPEG files with $60 \%$ quality and $50 \%$ size for quantification using Fiji software. Confocal images were directly uploaded in Fiji.

\section{Cell counting}

Firstly, a ROI, such as the cortex or the hippocampus, were defined by hand using the lasso tool. To further analyze numbers of cells and plaques the cell counter plugin by Kurt De Vos for Image $J$ was used. To distinguish plaque associated lba1+ microglia (PAM) from plaque distant lba1+ microglia, I programmed a macro to enlarge the marked plaque borders by $50 \mu \mathrm{m}$. All cells within this enlargement were counted as PAM whereas all cells outside the mark were defined as nonplaque associated cells. Cell and plaque counts were normalized to the analyzed area of the ROI.

\section{Percent area (\% area)}

The 16-bit confocal or slide scanner images were converted to 8-bit files to analyze the proportion a specific signal above a defined threshold within a ROI. This measurement was called \% area. For this purpose a threshold was set to remove noise and to only measure specific signal, independent of its fluorescence intensity. The threshold was kept constant with only minimal changes in case of high signal-to-noise ratio in an individual sample. The converted image showed a binary black and white image. The ratio of black to white signal within this image was measured with the fraction area setting of ImageJ.

\section{A $\beta$-plaque morphology}

For the analysis of plaque composition by Thioflavin $\mathrm{S}, \mathrm{A} \beta_{42}$ and $6 \mathrm{E} 10$ co-staining, the $z$-stack files imaged with the LSM700 were directly uploaded to Fiji. To study the spatial plaque morphology, all z-stacks were converted into a maximum projection. For every channel the outer border of the signal / plaque was marked with the lasso tool. The fluorescence intensity, the diameter of the plaque and the $6 \mathrm{E} 10$ fluorescence intensity were measured of at least 5 plaques per animal in a section of the prefrontal cortex layer $\mathrm{V}$. To calculate the halo of the plaque, which represents loosely attached $A \beta$-fibrils that are not condensed into a ThioS+ dense core, the $6 \mathrm{E} 10$ diameter was subtracted from the Thios diameter.

\section{Quantification of intralysosomal $A \beta$}

To quantify the degree of $A \beta$ digestion by lysosomes within microglia and astrocytes single z-planes of LAMP1 / A $\beta_{42}$ / Iba1a and LAMP1 / A $\beta_{42}$ / GFAP stainings with 40x magnification of the LSM700

$$
\text { 47| P a g e }
$$


were analyzed. To generate a ROI covering all cells (lba1+ or GFAP+) a constant threshold was set to label the morphology of the cells. These thresholds were converted into a mask and selected as ROI. The same way a second ROI for LAMP1+ lysosomal structures was generated. To measure the intensity of $A \beta$-signal in lysosomes, the lysosomal $R O I$ was applied to the $A \beta_{42}$ signal and the outside of the ROI was cleared. The resulting image showed $A \beta$-signal exclusively within lysosomes. This image was further processed by adding the cell-specific ROI and clearing the outside of the ROI. The fluorescence intensity of the $A \beta_{42}$ signal was measured, to show all $A \beta$ signal within lysosomes in the selected cell type. To double-check also the relative amount of $A \beta$ signal was calculated. Therefore, the whole procedure was done with the threshold-processed A $\beta$ image. In both cases the results were normalized to the numbers of cells or to the area in $\mathrm{mm}^{2}$.

\subsubsection{Imaris}

Imaris was used to analyze the degree of neuronal loss, to quantify the volumes of LAMP1+ lysosomes and CD68+ vesicles as well as to analyze microglia morphology using z-stack images obtained by confocal microscopy for three dimensional reconstruction.

\section{Analysis of neuronal loss (NeuN staining)}

For neuronal loss quantification a customized macro was written by Dr. Manuel Schölling from the Image and Data Analysis Facility, DZNE, Bonn, to allow the quantification of the signal in a defined ROI. The ROI (either the cortex starting at the medial line until lateral to the CA3 region, or the subiculum) was marked using the lasso tool. Running the macro the ROI was filled with a green color, simulating a second channel. This newly generated two-channel image was uploaded into Imaris. A size and intensity threshold was set for the NeuN signal. All spheres above these thresholds were shown using the surface tool. Since unspecific signal from blood vessels or damaged tissue could result in false positive signals, all sections were checked and corrected accordingly by hand. Then the number of all NeuN+ spheres within the green-colored ROI was calculated by the software. To normalize the cell number to the analyzed area and the analyzed volume, these parameters were also calculated from the ROI.

\section{Determination of CD68 and LAMP1 volumes in activated microglia}

To analyze the volume of CD68+ or LAMP1+ vesicles within Iba1+ microglia, confocal obtained $z$-stacks were uploaded in Imaris. Single lba1+ cells were selected for analysis. The surface tool was selected and a threshold for CD68 or LAMP1 signal intensity was set. A filter was applied to only select signal within the cell soma of the lba1+ cell. The average volume $\left[\mu^{3}\right]$ of the CD68+ or LAMP1+ vesicles was used for quantification. 


\section{Analysis of the microglia morphology}

Confocal z-stacks of Iba1 stainings were used for determination of microglia morphology using the surface tool from Imaris. Single cells in close proximity (PAM) and apart from plaques (non-PAM) were individually selected. Setting a threshold for fluorescence intensity in the lba1 channel allowed labeling the morphology of lba1+ cells including their processes. To remove cellular processes from neighboring cells, an additional filter for size was applied. Volumes and surface area of the cell were calculated and the ramification index was calculated as marker for cell activation according to Plescher et al. (2018). Briefly, this unit free index defines the spherical shape of the cell with 1 representing a perfectly round cell without processes which is equivalent to an amoeboid shaped, activated microglia.

\subsubsection{Protein Biochemistry 2.2.7.1. Sequential Amyloid $\beta$ isolation}

Different soluble fractions of $A \beta$ were isolated according to Henecka and colleagues (2012). Therefore the weight of the snap-frozen tissue was determined and the protocol downscaled for the hippocampus. The tissue was homogenized in $100 \mu \mathrm{l}$ homogenization buffer using a Precellys 24-Dual Tissue homogenizer at maximum speed for $15 \mathrm{sec}$. Half of the homogenate was snap frozen and stored at $-80^{\circ} \mathrm{C}$ for further experiments.

The remaining $50 \mu \mathrm{l}$ of hippocampus homogenate were mixed with the equal volume of 2x RIPA buffer. Samples were sonicated for $10 \mathrm{~s}$ with $80 \%$ duty and $12 \%$ power, followed by cell lysis for 30 min on ice. Using an ultracentifuge with a TLA-55 rotor the samples were spun for 30 min at $100.000 \times \mathrm{g}$ at $4^{\circ} \mathrm{C}$. The supernatant (RIPA-soluble fraction) was collected and snap frozen. The RIPA-insoluble pellet was dissolved in $100 \mu \mathrm{l} 2 \%$ SDS in $25 \mathrm{mM}$ Tris- $\mathrm{HCl}$ ( $\mathrm{pH}$ 7.5). After sonication, the samples were heated for $5 \mathrm{~min}$ at $90^{\circ} \mathrm{C}$ and ultracentrifuged with the same settings. The supernatant was collected (SDS-soluble fraction) and snap frozen. The SDS-insoluble pellet was dissolved in $50 \mu \mathrm{l} 70 \%$ formic acid (FA) and sonicated. The pH was neutralized with $950 \mu \mathrm{l} 1 \mathrm{M}$ Tris $(\mathrm{pH} 11)$. The samples were snap-frozen. All fractions were stored at $-80^{\circ} \mathrm{C}$ until further usage.

\subsubsection{BCA assay}

To determine the protein concentrations within the latter described soluble hippocampal fractions, $\mathrm{BCA}$ assays were done according to manufacturer's instructions. Briefly, RIPA fractions were vortexed, while the SDS-fractions were heated for $5 \mathrm{~min}$ at $90^{\circ} \mathrm{C}$, sonicated for $10 \mathrm{~s}$ with $80 \%$ duty and $12 \%$ power and spun at $13.000 \times \mathrm{g}$ for $2 \mathrm{~min}$, before use. To measure the total protein concentration RIPA and SDS fractions were pre-diluted 1:10 with the respective buffers of preparation. These buffers were also used for standard preparation and as blank. 
For the assay samples were analyzed as duplets. Briefly, $25 \mu \mathrm{l}$ of sample was added per well to a 96 well plate. $200 \mu \mathrm{l}$ of provided detection solution (Buffer A: Buffer B used in a 50:1 ratio) was added per well. The plate incubated at $37^{\circ} \mathrm{C}$ in the dark with gentle shaking, prior to plate reading with FLUOstar omega plate reader at $562 \mathrm{~nm}$. Protein concentrations were calculated using Excel.

\subsubsection{Meso Scale Discovery ${ }^{\mathrm{TM}}$ (MSD) Electrochemi- luminescence}

For this study the V-PLEX A $\beta$ Peptide Panel 1 (6E10) Kit from Meso Scale Discovery ${ }^{\mathrm{TM}}$ (MSD) was used. MSD is an electrochemiluminescence (ECL) assay combining sandwich enzyme-linked immunosorbent assay (ELISA) with the high sensitivity of ECL based detection. Hence it provides very sensitive detection over a broad concentration range. Due to position-defined coating of $A \beta$ species specific capture antibodies, simultaneous measurements of $A \beta_{38}, A \beta_{40}$ and $A \beta_{42}$ within one sample are possible. To measure the concentrations of the individual $A \beta$ species within the fractions obtained by sequential $A \beta$ isolation described in 2.2.7.1, the samples were pre-diluted in the provided buffer Diluent 35. For samples obtained from 3 month old animals the following dilutions were used: RIPA fractions: 1:30; SDS fractions: 1:50; formic acid fractions: 1:300. For samples of 9 month old animals samples were diluted 1:100 (RIPA fractions), 1:300 (SDS fractions), 1:750 (formic acid fractions) and 1:4 (plasma).

The assay was performed according to manufacturer's instructions. Briefly, the provided pre-coated 96-well plates were washed three times with $150 \mu$ I PBST (PBS supplemented with $0.025 \%$ Tween20) at $300 \mathrm{rpm}$ shaking. To avoid unspecific binding, the wells then were blocked with $150 \mu$ l Diluent 100 for $1 \mathrm{~h}$ at $\mathrm{RT}$. $A \beta_{38}, A \beta_{40}$ and $A \beta_{42}$ standard and pre-diluted samples were applied in duplicates to the plate and incubated for $2 \mathrm{~h}$ at RT at $300 \mathrm{rpm}$. Then the plate was washed three times with PBST, before $150 \mu \mathrm{l}$ of Read Buffer T was added and the plate was read at $620 \mathrm{~nm}$ with the Sector Imager 6000. The data was transferred to MSD Discovery Workbench 3.0 Data Analysis Toolbox and further analyzed with Excel.

\subsubsection{SDS-PAGE}

To analyze proteins according to their molecular weight, sodium dodecyl sulfate polyacrylamide gel electrophoresis (SDS-PAGE) was used. Two-layered polyacrylamid gels, containing an upper stacking part (1/3) and a lower resolving part (2/3), were cast using the Bio-Rad Mini-PROTEAN® Tetra electrophoresis system. Gels were prepared according to the protocol in Table 19. 
Table 19: Protocol for one SDS gel composed of $12 \%$ resolving gel and $4 \%$ stacking gel.

\begin{tabular}{llll}
\hline \hline Compounds & Resolving gel & Compounds & Stacking gel \\
\hline \hline Resolving Buffer & $1.30 \mathrm{ml}$ & Stacking Buffer & $500 \mu \mathrm{l}$ \\
$\mathrm{ddH}_{2} \mathrm{O}$ & $1.66 \mathrm{ml}$ & ddH $_{2} \mathrm{O}$ & $1.21 \mathrm{ml}$ \\
$30 \%$ Acrylamide & $2.04 \mathrm{ml}$ & $30 \%$ Acrylamide & $540 \mu \mathrm{l}$ \\
$10 \%$ SDS & $50 \mu \mathrm{l}$ & $10 \%$ SDS & $20 \mu \mathrm{l}$ \\
APS & $50 \mu \mathrm{l}$ & APS & $20 \mu \mathrm{l}$ \\
TEMED & $2 \mu \mathrm{l}$ & TEMED & $3 \mu \mathrm{l}$ \\
\hline \hline
\end{tabular}

For equal protein concentrations SDS samples from paragraph 2.2.7.1 were diluted with $2 \%$ SDS buffer to a final concentration of $1 \mathrm{mg} / \mathrm{ml}$. Samples were mixed with $5 x$ sample buffer and heated for $5 \mathrm{~min}$ to $95^{\circ} \mathrm{C}$ to denature the proteins. Per gel $15 \mu \mathrm{g}$ protein per sample were loaded. Samples were separated by running the gel for about $180 \mathrm{~min}$ at $120 \mathrm{~V}$. For a reference of the molecular weight, protein marker PageRuler® Plus Prestained Protein Ladder was used.

\subsubsection{Western Blotting}

The SDS gel was transferred to a nitrocellulose membrane using the Mini-Trans Blot cell set up according to the manufacturer's protocol. Blotting was achieved at $100 \mathrm{~V}$, for $1 \mathrm{~h}$ at $4^{\circ} \mathrm{C}$. To check for successful protein transfer from the gel to the membrane, Ponceau $S$ staining was done. Therefore the membrane was briefly exposed to $0.1 \%[\mathrm{w} / \mathrm{v}]$ PonceauS in $5 \%[\mathrm{v} / \mathrm{v}]$ acetic acid. To decolorize, the membrane was washed with tab water, until no red dye was visible anymore.

To reduce unspecific antibody binding, membranes were blocked with $4 \%$ non-fat dried milk powder in $0.025 \%$ PBST for $1 \mathrm{~h}$ at RT. Primary antibodies were diluted according to the Table 7 in $1 \%$ non-fat dried milk powder in $0.025 \%$ PBST and incubated $\mathrm{ON}$ at $4{ }^{\circ} \mathrm{C}$. After 3 washing steps of 10 min each with $0.025 \%$ PBST, membranes were incubated with species-specific secondary horse-radish peroxidase (HRP) coupled antibodies listed in Table 8 for $1 \mathrm{~h}$ at RT. Membranes were washed again 3 times for $10 \mathrm{~min}$ with $0.025 \%$ PBST. To detect chemiluminescence equal amounts of ECL substrate was mixed and was added to the membrane. After a brief incubation time of 1 min, chemiluminescence signal was detected with the ChemiDoc ${ }^{T M}$ MP. After chemiluminescence was detected the marker was imaged, while keeping the membrane at the same position. This image was merged to the image of the chemiluminescence.

In case of weak signals, Amersham ${ }^{\mathrm{TM}}$ ECL Prime Western Blotting Detection Reagent was added as substrate for higher sensitivity.

To re-stain a membrane e.g for a house-keeper protein, the membrane was washed 3 times for 15 min with $0.025 \%$ PBST, prior to blocking and re-incubation with another primary antibody.

For quantification of protein levels, signal intensity of the bands were measured in a 16-bit image with 1200 dpi using Fiji software. The intensity of the protein of interest was normalized to the intensity of a house-keeper protein of the individual sample. 


\subsubsection{Olink® MOUSE EXPLORATORY Analysis service}

Olink ${ }^{\circledR}$ provides a Proximity Extension Assay (PEA)-based platform screening for 92 biomarkers in mouse samples. For this assay RIPA fractions obtained by sequential $A \beta$-isolation (see section 2.2.7.1) were used. The total protein concentration was measured by BCA assay as described before and adjusted to $1 \mu \mathrm{g} / \mu \mathrm{l}$. Samples were shipped on dry ice to Olink® and analyzed by the company.

The assay uses two complementary oligonucleotide-labeled antibodies (probes) specifically binding to the target protein. Thereby nucleotide-hybrids are built in case of close proximity. These hybrids are amplified by DNA polymerization and quantified by real-time PCR. The multiplex-assay allows for simultaneous analysis of 92 proteins. The proteins screened included markers for inflammation, chemotaxis and cell mortality.

\subsubsection{Ethics Statement}

All experiments were conducted as approved by the Government of Lower Saxony, Germany. The Approval ID is G14-1738.

Animals with the genotype Cre ${ }^{\mathrm{ERT} /+}$ Rab7 fl/fl $5 x F A D$ het mice were used for all experiments. They were housed under standard conditions in ventilated cages with 2-5 animals each. Animals were held in temperature-controlled and air pressure controlled environment on a $12 \mathrm{~h}$ light/dark cycle with food and water available ad libitum.

\subsubsection{Statistics}

For statistical analysis GraphPad Prism ${ }^{\circledR} 6$ (GraphPad Software, Inc.) was used. To compare

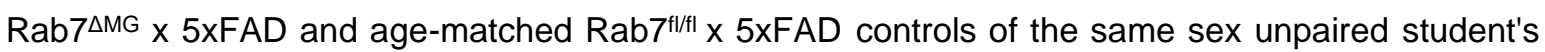
$t$-test was used. A $p$ value of $<0.05$ indicated statistical significance. All values are represented as mean \pm SEM. $N$ is the number of analyzed samples from the same biological sample, $n$ is the number of biological samples. 


\section{Results}

\subsection{Generation and validation of the Rab7 $7^{\Delta M G} x$ 5xFAD mouse model}

To investigate the degradation capacities of microglia on amyloid burden in vivo, the triple transgenic mouse model Rab7 $\triangle M G$ x 5xFAD was generated. The microglia-specific $\mathrm{KO}$ of the endolysosomal GTPase Rab7 (Rab7 ${ }^{\triangle M G}$ ) was induced by i.p. administration of tamoxifen at P21 as illustrated in Figure 13. To investigate the role of this cellular pathway on amyloid pathology these mice were further crossed with the 5xFAD mouse model, overexpressing human APP harboring 3 FAD mutations and PSEN1 with two FAD mutations. This triple transgenic mouse line was called Rab7 $\triangle M G$ x $5 \times F A D$. Animals with tamoxifen induced Cre-recombination will be referred to as Rab7 ${ }^{\triangle M G} \times 5 \times F A D$ (labeled in red) and sham treated control animals will be addressed as Rab7 $7^{\mathrm{flfl}} \mathrm{X}$ 5xFAD (labeled in blue).
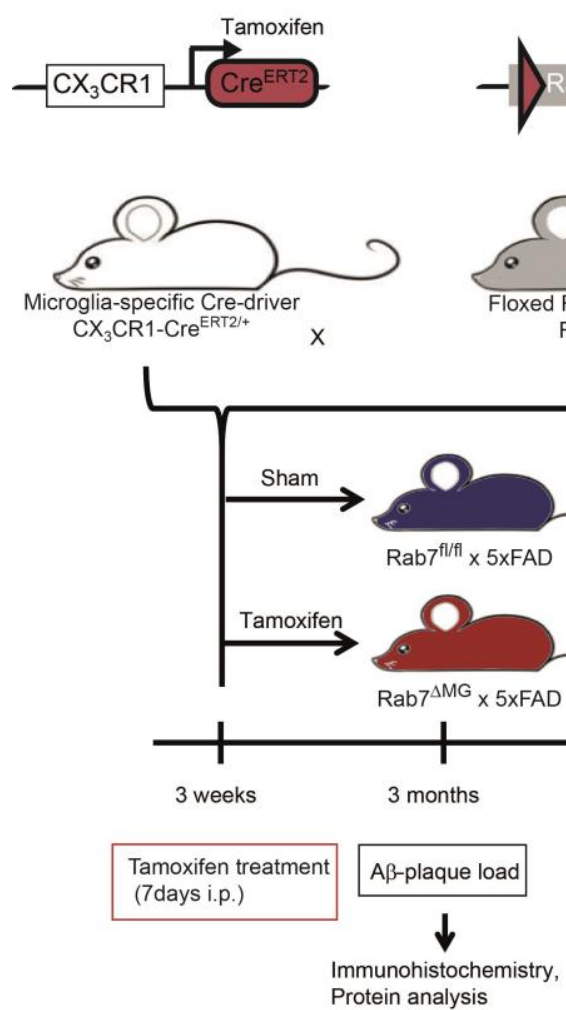

Figure 13: Experimental setup.

To generate the Rab7 ${ }^{\Delta M G} \times 5 \times F A D$ mouse line animals with the following genotype were crossed: Cre ${ }^{\mathrm{ERT} 2 /+}$ Rab7 ${ }^{\mathrm{fl} / \mathrm{fl}}$ and $5 x F A D$ het. To induce the microglial KO of Rab7 (Rab7 ${ }^{\triangle M G}$ ) animals were i.p. injected at P21 with tamoxifen dissolved in corn oil for 7 consecutive days (Rab7 ${ }^{\triangle M G} \times 5 \times F A D$ ). Littermate control animals were injected with the same dose of corn oil (Rab7 $\left.7^{\mathrm{flfl}} \times 5 \times \mathrm{FAD}\right)$. The first cohort was dissected at 3 months of age, the second cohort was sacrificed at 9 months of age. From both cohorts brain tissue was collected and was used either for protein biochemistry or for Golgi-Cox analysis and for immunohistochemistry.

53| P a g e 
The Rab7 $\triangle M G$ x 5xFAD mouse line was investigated at two different time-points: one cohort was investigated at 3 months which is the earliest time point of $A \beta$-plaque deposition and the second cohort was investigated at 9 months of age when neuronal loss was observed in the 5xFAD mouse line (Oakley et al., 2006).

To our knowledge, there is no antibody against Rab7 available which is suitable for immunohistochemistry. Hence, Rab7 ${ }^{\triangle M G}$ was validated by immunohistochemistry staining the endolysosomal markers CD68 and LAMP1. To verify degradation impairment we quantified the volumes of CD68+ and LAMP1+ endo-lysosomes as well as A $\beta$-peptide accumulations in these endolysosomes.

\subsubsection{Microglia of $R^{2} 7^{\triangle M G} \times 5 x F A D$ mice showed increased CD68 positive endo-lysosomes}

Immunohistochemical analysis of a co-staining for CD68, Iba1 (lonized calcium-binding adaptor molecule 1 or allograft inflammatory factor 1 (AIF-1)) as a microglial marker and 6E10 which binds to the N-terminus of APP was done (Figure $14 \mathrm{~A}$ ). Given that CD68 is also a marker for microglia activation, which is upregulated in aging and in disease, $A \beta$-plaque distant Iba1-positive $(+)$ microglia were selected for analysis to assess for enlarged lysosomes in microglia from Rab7 ${ }^{\Delta M G} \mathrm{X}$ 5xFAD mice. The CD68 volume within these cells was measured using Imaris software for 3D reconstruction. The analysis showed significantly bigger CD68 vesicles in Iba1+ microglia of 9 month old Rab7 ${ }^{\triangle M G} \times 5 \times F A D$ animals compared to Rab7 $7^{\text {flffl }} \times 5 \times F A D$ controls (Figure 14 B).
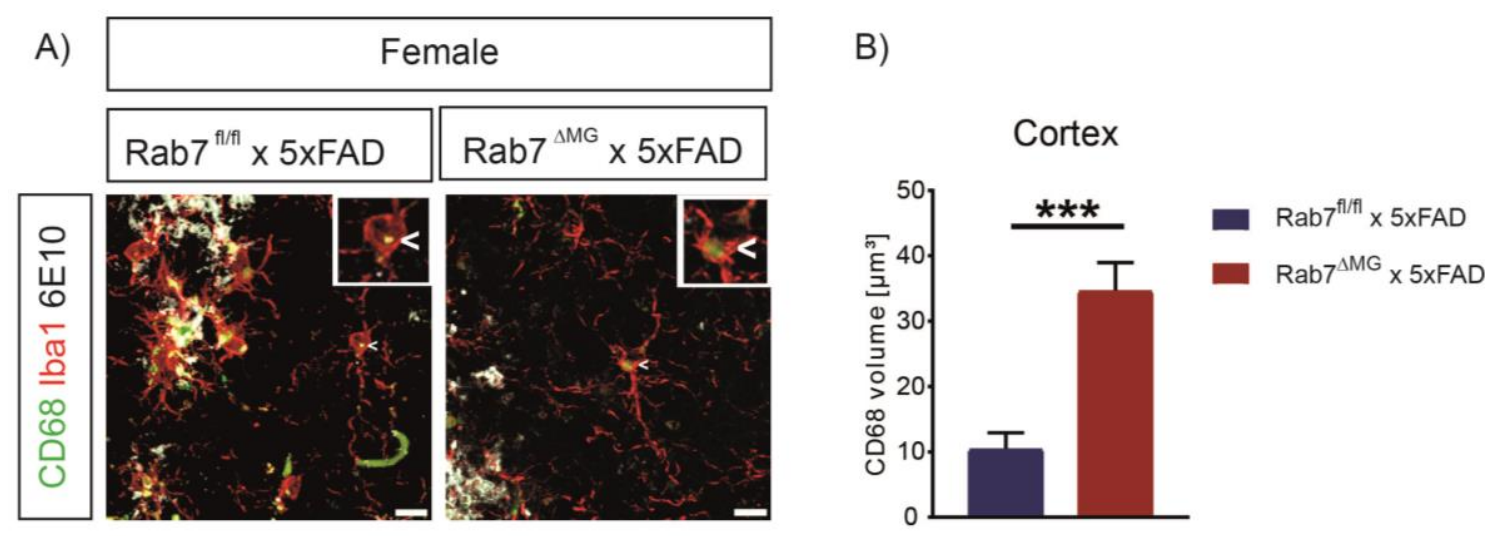

Figure 14: CD68 volumes were strongly increased in Iba1+ microglia.

A) Confocal images of immunohistochemistry for CD68 (green), Iba1 (red) and 6E10 (white) on brain tissue of 9 month old Rab7 $7^{\mathrm{fl} / \mathrm{fl}} \times$ 5xFAD (left) and Rab7 $7^{\triangle \mathrm{MG}} \times 5 \times$ XAD (right) females. Arrowhead (<) indicates CD68-positive (+) vesicles inside Iba1+ microglia. The selected cell is magnified in the upper right corner. Scale bar: $10 \mu \mathrm{m}$. B) Quantification of average CD68 volume $\left[\mu^{3}\right]$ in Iba1+, A -plaque (6E10) distant cells within cortex layer V-VI shows significant enlargement of CD68 in the Rab7 $7^{\triangle M G} \times 5 \times F A D$ animals (red) compared to the Rab7 $7^{\mathrm{fl} / \mathrm{fl}} \times 5 \times \mathrm{FAD}$ controls (blue). Data are presented as mean \pm SEM. $n \geq 6$ animals, $N=10$ cells per animal. Unpaired student's t-test. ${ }^{* * *} p<0.001$. 


\subsubsection{Enlargement of LAMP1 positive lysosomes were found in $\mathrm{Rab} 7^{\triangle \mathrm{MG}} \times 5 \times \mathrm{xFA}$ mice}

To further confirm the $\mathrm{KO}$ of Rab7 in microglia of Rab7 ${ }^{\triangle M G} \times 5 \times F A D$ mice, immunohistochemistry for the lysosomal marker LAMP1 (lysosomal-associated membrane protein) in Iba1+ microglia was done (Figure 15). By disrupting the fusion of the late endosome with the lysosome in Rab7 $\triangle \mathrm{MG}$ animals, the lysosomes are functionally impaired, accumulate and increase within the cell over time (Safaiyan et al., 2016). Rab7 $\triangle M G$ x 5xFAD animals showed larger volumes of LAMP1+ lysosomes within Iba1+, A -plaque distant microglia (Figure $15 \mathrm{E}-\mathrm{I}$ ) compared to control Rab7 ${ }^{\mathrm{fl} / \mathrm{ll}} \times 5 \times \mathrm{FAD}$ animals at 9 months of age (Figure 15 A-D; I). We analyzed A $\beta$-plaque distant microglia, to measure the basal lysosomal activity in the model, independent of immediate physical contact to $A \beta$-plaques.

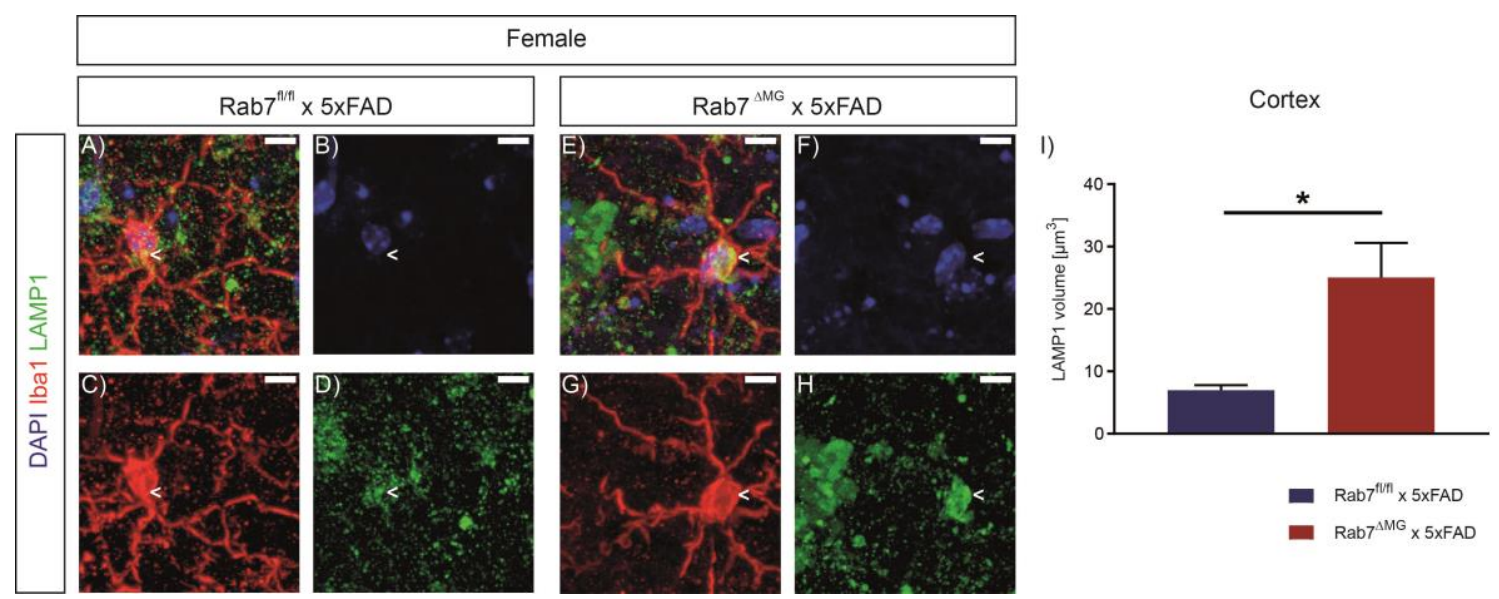

Figure 15: LAMP1+ lysosomes were enlarged in Rab7 $7^{\triangle M G} \times 5 \times F A D$ microglia.

$\mathbf{A}+\mathbf{E})$ Merge images of immunohistochemical co-stainings for $\mathbf{B}+\mathbf{F}$ ) nuclei stained with DAPI (blue), $\mathbf{C}+\mathbf{G}$ ) microglial marker lba1 (red) and $\mathbf{D}+\mathbf{H}$ ) lysosomal marker LAMP1 (green). Images were obtained by confocal microscopy. Scale bar: $10 \mu \mathrm{m}$. I) Quantification of LAMP1 volume $\left[\mathrm{mm}^{3}\right]$ in Iba1+ microglia was assessed by Imaris software. $\mathrm{N} \geq 10$ cells of $\mathrm{n} \geq 7$ females at 9 months of age were analyzed. Data are presented as mean \pm SEM. Statistically significant differences

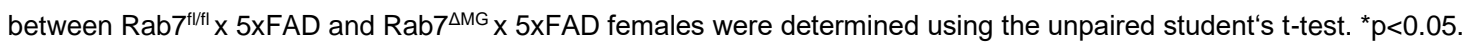

\subsection{3. $A \beta$ degradation is disturbed in $R a b 7^{\triangle M G} \times 5 \times F A D$ mice}

Given that microglia are the main phagocytes of the brain which are highly active in removing $A \beta$-peptides in $A D$, validation of the functional deficit in the endo-lysosomal pathway in Rab7 $7^{\triangle M G} x$ 5xFAD mice in terms of $A \beta$ clearance was done using immunohistochemistry for LAMP1, $A \beta_{42}$ and Iba1 (Figure $16 \mathbf{A}-\mathrm{H}$ ). Quantification revealed doubled fluorescence intensity of $A \beta_{42}$ within LAMP1+ vesicles inside lba1+ microglia (indicated by <) verifying significant accumulation of $A \beta_{42}$ 
inside microglia of Rab7 ${ }^{\triangle M G}$ x 5xFAD (Figure $16 \mathrm{~K}$ ). Measuring the relative area occupied by $A \beta_{42}$ inside microglial lysosomes supported this finding (Figure $16 \mathrm{~L}$ ). In addition to the findings of enlarged CD68+ and LAMP1+ vesicles, we concluded that Rab7 ${ }^{\triangle M G} \times 5 \times F A D$ mice indeed showed functional impairment of the endo-lysosomal apparatus in microglia in terms of $A \beta$ degradation.

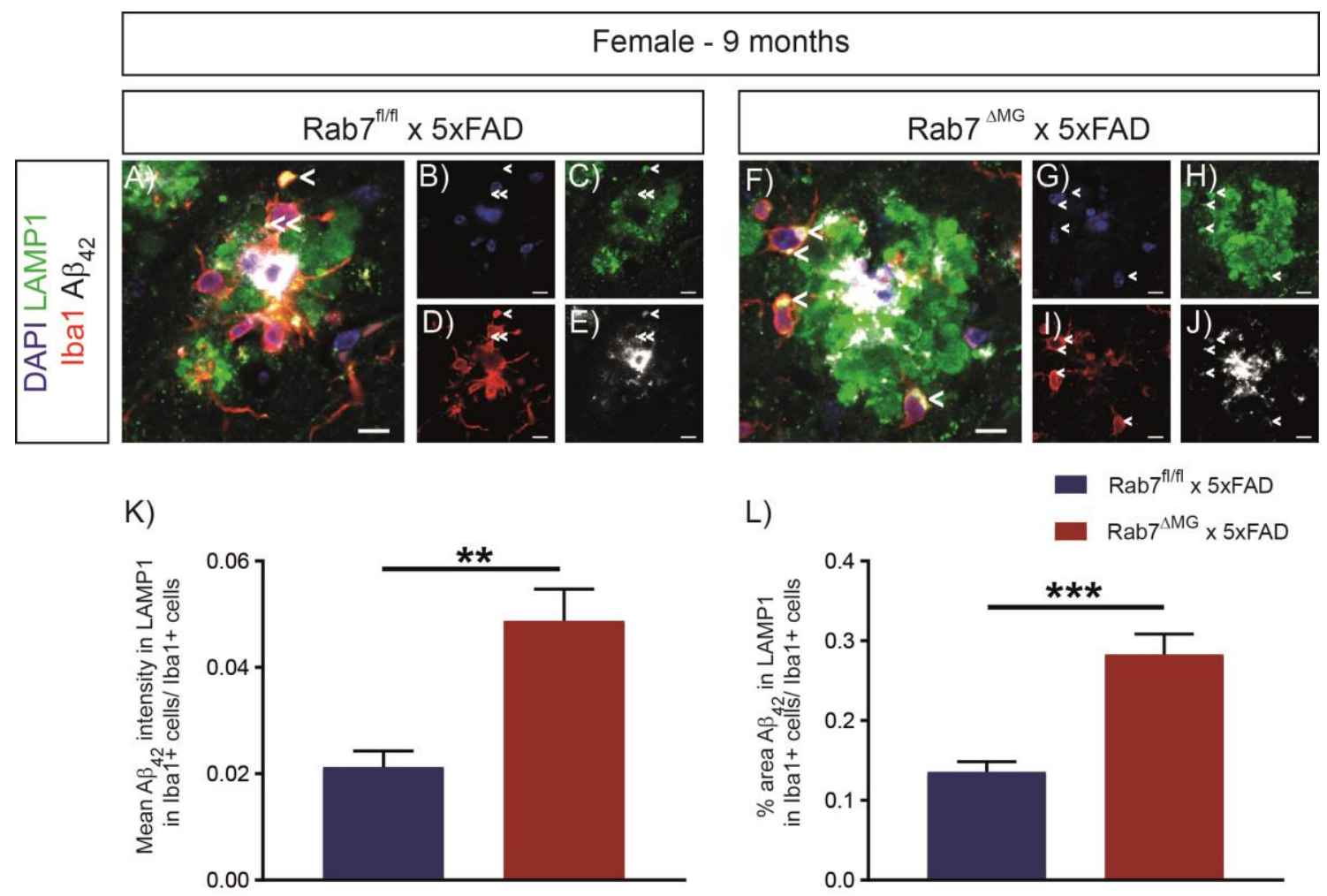

Figure 16: $A \beta$ clearance was disrupted in Rab7 ${ }^{\triangle M G}$ x 5xFAD microglia.

A-J) Fluorescence staining for LAMP1 (green) and lba1 (red) and $A \beta_{42}$ (blue) on brain sections of 9 month old Rab7 ${ }^{f / l|l|} x$ 5xFAD (left) and Rab7 ${ }^{\triangle M G}$ x 5xFAD (right) females. A+F) Merge images of C+H) LAMP1 (green), D+l) lba1 (red), $\mathbf{E}+\mathbf{J}) A \beta_{42}$ (white) stainings and $\mathbf{B + G}$ ) nuclei stained with DAPI (blue) are shown. One confocal plane was imaged as tile scan and all Iba1+ cells within the cortical layers I-VI were analyzed. Scale bar: $10 \mu \mathrm{m}$. K) Quantification of $A \beta_{42}$ fluorescence intensity within LAMP1+ vesicles inside lba1+ cells is shown. L) Quantification of the relative area positive for $A \beta_{42}$ inside microglial LAMP1+ vesicles. Data are shown as mean \pm SEM. $n \geq 9$ animals, $N \geq 72$ cells per animal. Unpaired student's t-test. ${ }^{* *} p \leq 0.01,{ }^{* * *} p \leq 0.001$.

\subsection{Plaque pathology in the Rab7 ${ }^{\triangle M G} \times 5 \times F A D$ mice was reduced}

After characterizing the properties of Rab7 ${ }^{\triangle M G}$ impaired microglia in 5XFAD mice, the next question I addressed was whether $A \beta$-plaque pathology was altered in these animals compared to Rab7 $7^{\text {fl/fl } x}$ 5xFAD littermates. Microglia are highly phagocytic active in $A D$ clearing extracellular $A \beta$ 
(Frackowiak et al., 1992; Wisniewski et al., 1991). Therefore, we hypothesized that disruption of $A \beta$-degradation after uptake in Rab7 ${ }^{\triangle M G} \times 5 \times F A D$ mice would cause reduced $A \beta$-clearance and thereby lead to increased $A \beta$-plaque burden. To investigate this, the numbers of $A \beta$-plaques, their architecture and composition were analyzed.

\subsubsection{Microglial Rab7 $\triangle \mathrm{MG}$ reduces $A \beta$-plaque numbers throughout disease progression in females of $5 x F A D$}

To investigate whether disruption of the Rab7-dependent degradation pathway in microglia resulted in changes in the $A \beta$-plaque load in Rab7 ${ }^{\triangle M G} \times 5 \times F A D$ mice, immunohistochemistry for $A \beta$ using a $6 \mathrm{E} 10$ antibody was done (Figure 17). The A $\beta$-plaque numbers in the cortex and the hippocampus were counted in 3 and 9 month old females and males, respectively. Whereas there was no significant difference detectable in the $A \beta$-plaque load in the young females, the aged Rab7 ${ }^{\triangle M G} x$ 5xFAD females showed significantly less $A \beta$-plaques compared to age-matched Rab7 ${ }^{\text {fl/fl }} \times 5 \times F A D$ controls in the cortex (Figure $17 \mathrm{E})$. In the hippocampus a nearly significant reduction $(p=0.0516)$ of $6 \mathrm{E} 10+\mathrm{A} \beta$-plaques was found in 9 month old Rab7 ${ }^{\mathrm{MG}} \times 5 \times \mathrm{FAD}$ compared to $\mathrm{Rab}^{\mathrm{fl} / \mathrm{fl}} \times 5 \times \mathrm{FAD}$ controls (Figure 17 F). This effect was surprising, since we hypothesized to find more A $\beta$-plaques in Rab7 ${ }^{\triangle M G} \times 5 \times F A D$ animals, due to their impairment in protein clearance. This effect was only observed in females though. No differences in $6 \mathrm{E} 10+A \beta$-plaques numbers were found in 3 or 9 month old males (Figure $17 \mathbf{K}+\mathbf{L}$ ). Moreover, $A \beta$-plaque load was in general lower in males compared to females of the same age. Therefore we concluded that Rab7 $\triangle \mathrm{MG} \times 5 \times \mathrm{FAD}$ had a sex-dependent effect on $A \beta$-plaque formation resulting in fewer $A \beta$-plaques in Rab7 ${ }^{\triangle M G} \times 5 \times F A D$ females compared to Rab7 $7^{\text {fl/fl }} \times 5 \times$ XFA females while there was no effect detectable in males. 


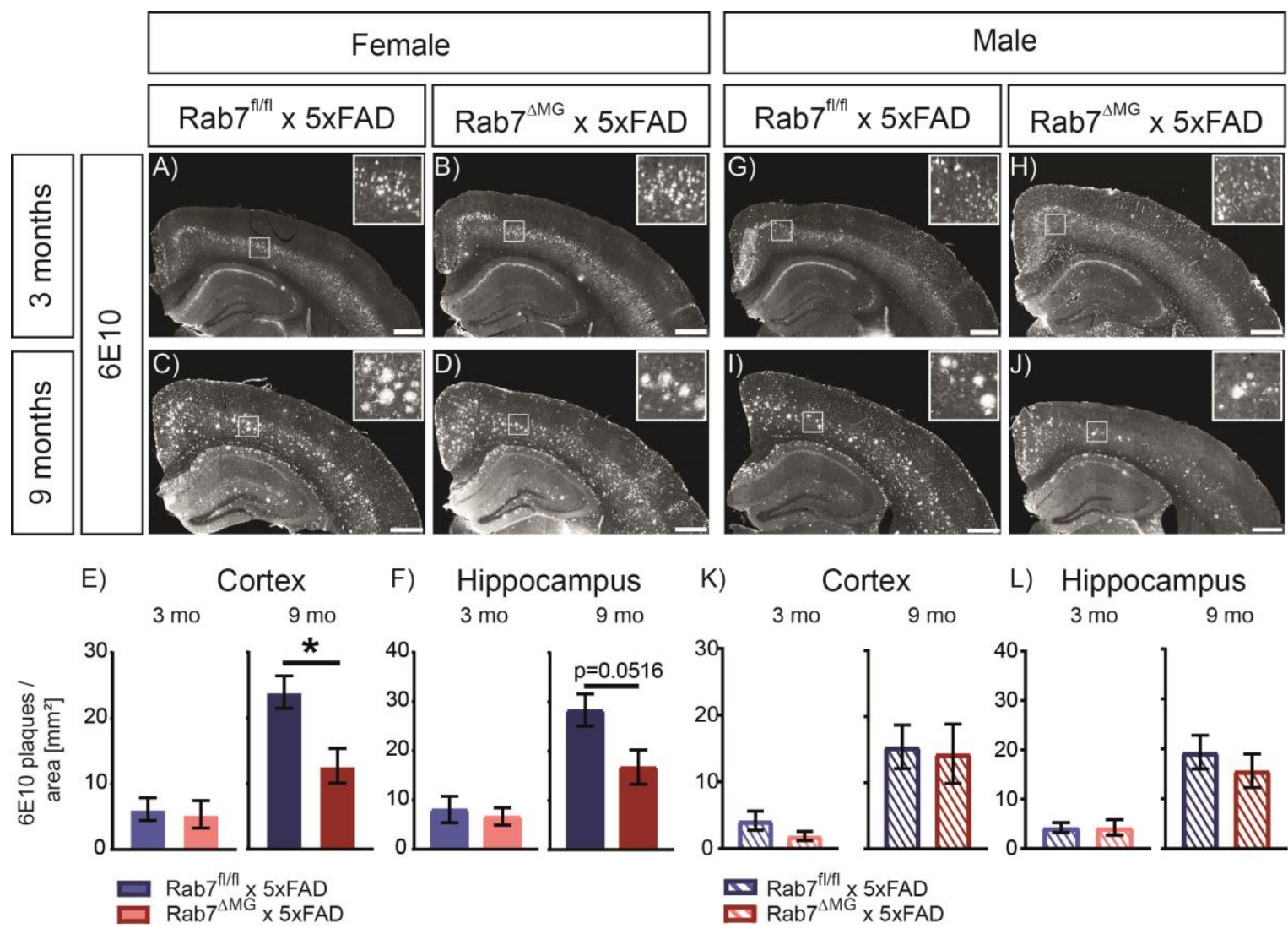

Figure 17: A $\beta$-plaque numbers were reduced in aged $R a b 7^{\triangle M G} \times 5 \times F A D$ females.

A-B) Representative fluorescence staining for $6 \mathrm{E} 10$ (white) on brain sections of of 3 month old Rab7 $7^{\text {fl/fl }} \times 5 \times F A D$ and $\mathrm{Rab}^{\triangle \mathrm{MG}} \times 5 \times \mathrm{xFAD}$ females. C+D) Staining on tissue of 9 month old females. Images were obtained by automated microscopy using the Axioscan Z1. Scale bar: $500 \mu \mathrm{m}$. E) Quantification of 6E10 positive plaques / area [mm²] within the cortex of 3 month old females (left) and of 9 month old females (right). F) Analysis of plaque numbers / $\mathrm{area}^{\left[\mathrm{mm}^{2}\right]}$ within the hippocampus by 3 and 9 months. G-H) Histological stainings for 6 E10 on brain tissue of 3 month old old Rab7 $7^{\text {fl/fl }} \times 5 \times$ XAD and Rab7 ${ }^{\triangle M G} \times 5 \times F A D$ males. I+J) Equivalent staining on samples of 9 month old males. K) Quantification of 6E10+ plaque numbers / area [mm²] within the cortex of 3 month (left) and 9 month (right) males. L) Quantifiaction of plaque numbers within the hippocampus. Control Rab7 $7^{\mathrm{f} / \mathrm{fl}} \times 5 \times \mathrm{FAD}$ animals are shown in blue ( 3 months) or dark blue ( 9 months) and Rab7 $7^{\triangle M G} \times 5 x F A D$ are presented in pink ( 3 months) or red ( 9 months). Filled graphs represent the female cohorts, striped bars show male cohorts. Data are shown as mean \pm SEM. $n \geq 8$ animals with $\mathrm{N}=2$ sections / animal for both sexes at 3 month cohorts. $\mathrm{n} \geq 4$ animals with $\mathrm{N} \geq 3$ sections / animal for both sexes at 9 month cohorts. Unpaired student's t-test. ${ }^{*} p \leq 0.05$. Analysis of 3 month cohort was done by Michaela Limmer. 


\subsubsection{A $A$-plaques were altered in morphology and composition}

We wondered whether the spatial morphology and composition of mature $A \beta$-plaques were altered due to Rab7 KO in 9 month old Rab7 ${ }^{\triangle M G} \times 5 \times F A D$ mice. Therefore I did immunohistochemical costainings for $A \beta_{42}, 6 E 10$ as a general marker of $A \beta$ and APP, and for ThioflavinS (ThioS) to label the dense core of $A \beta$-plaques (Figure 18). To rule out $A \beta$-plaque overlap, due to high plaque density, $A \beta$-plaques within cortex layer $V$-VI were analyzed. We found significantly smaller $A \beta$-plaques in Rab7 $^{\triangle M G}$ x 5xFAD females (Figure 18 A-D). More precisely, these $A \beta$-plaques featured smaller dense cores (Figure $18 \mathrm{~B}$ ) and smaller overall size (Figure $18 \mathrm{C}$ ). The A $\beta$-plaque halos, which are defined as $A \beta$-peptides of a plaque clustering around the dense core, were significantly smaller in females (Figure $18 \mathrm{D}$ ). We found the halos of the $A \beta$-plaques were more heterogeneous, with areas which appeared to be less condensed in Rab7 $\triangle M G$ x $5 \times$ FAD females. Measuring the signal intensity of $6 \mathrm{E} 10$ to plaque area within the $A \beta$-plaque, we detected significantly more signal in Rab7 ${ }^{\triangle M G} \times 5 \times F A D$ females compared to Rab7 $7^{\mathrm{flfl}} \times 5 \times \mathrm{FAD}$ females due to the overall smaller plaquearea (Figure $18 \mathrm{E}$ ). By normalizing to $6 \mathrm{E} 10$ signal to the plaque number, we did not find differences (Figure $18 \mathrm{~F}$ ). These findings suggest for changes in the global $A \beta$-plaque architecture in 9 month old female Rab7 $\triangle M G$ x 5xFAD mice.

In male Rab7 $\triangle M G$ x 5xFAD mice $A \beta$-plaques did not show any difference in their sizes and architecture compared to $A \beta$-plaques in Rab7 fl/fl $\times 5 \times F A D$ males (Figure 18 G-L).

Consequently, the Rab7 $\triangle M G$ in $5 x F A D$ had a sex-dependent effect on $A \beta$-plaque composition. Hence, $A \beta$-plaque are smaller with changes in global morphology in Rab7 $\triangle \mathrm{MG} \times 5 \times \mathrm{xAD}$ females compared to Rab7 ${ }^{f / f 1} \times 5 \times F A D$ females at 9 months. 
A)

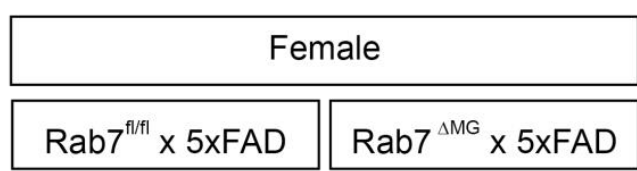

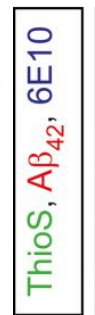

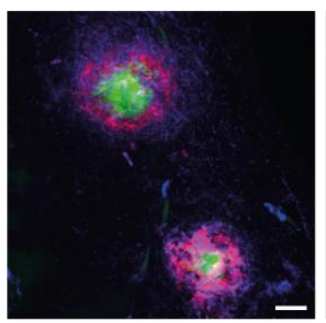

B)
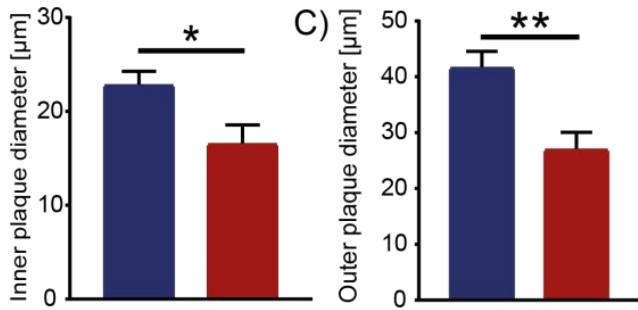

D)

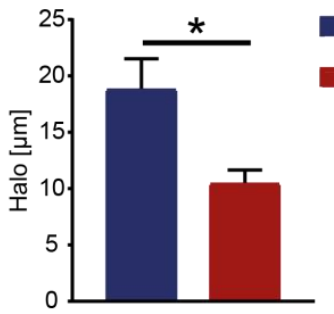

E)
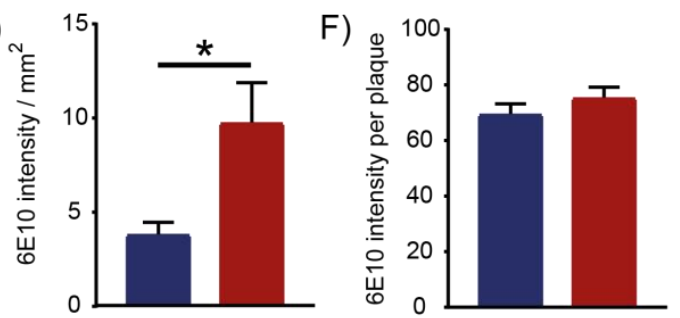

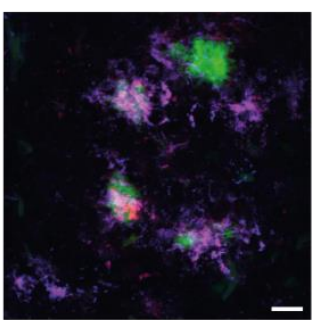

H)
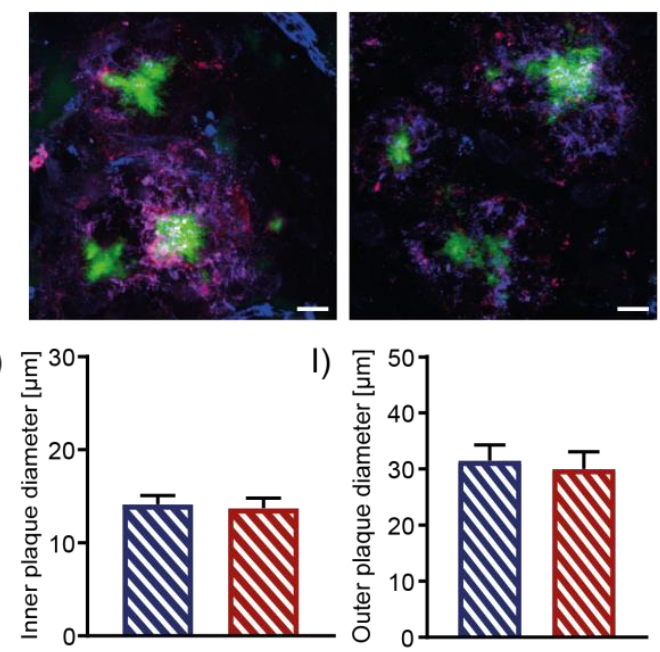

G)

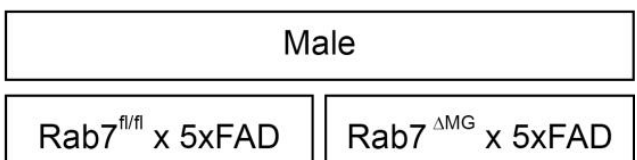

J)

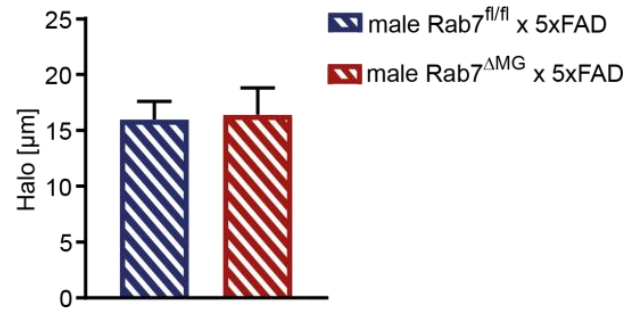

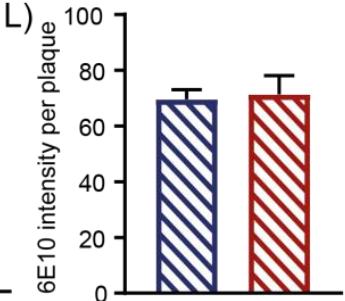

Figure 18: A $\beta$-plaques were smaller and showed altered morpholgy in Rab7 $7^{\triangle M G} \times 5 \times F A D$ females.

$A+G)$ Representative images of histological staining for ThioS (green), $A \beta_{42}$ (red) and 6E10 (blue) in 9 month old Rab7 $7^{1 / 1 / 1} \times 5 \times F A D$ (left) and Rab7 ${ }^{\triangle M G} \times 5 \times F A D$ (right) animals. Images were taken within cortex layer V-VI above the CA1 region using a confocal microscope with $63 x$ oil objective. Here representative maximum projections of $z$-stacks are shown. Scale bar: $10 \mu \mathrm{m} . \mathbf{B}+\mathbf{H}$ ) Inner plaque diameter defined by ThioS signal are shown. $\mathbf{C}+\mathbf{H}$ ) The average outer plaque diameter was measured according to $6 \mathrm{E} 10$ and $A \beta_{42}$ signals. D+J) The difference between the outer and the inner $A \beta$-plaque diameter gave information about the thickness of the halo. The halo is defined as the area of $A \beta$ peptides which do not belong to the ThioS+ dense core of an A -plaque. E+K) Fluorescence intensity of $6 E 10$ signal within the whole $A \beta$-plaque area was reduced due to smaller plaque size. $\mathbf{F}+\mathbf{K}$ ) When normalized to the plaque numbers, $6 \mathrm{E} 10$ fluorescence intensity did not show over all changes. Data are presented as mean \pm SEM. Rab $7^{1 / 1 / 1} x$ 5xFAD controls are shown in blue/blue stripes and Rab7 ${ }^{\mathrm{MMG}} \times 5 \times \mathrm{FAD}$ are displayed in red/red stripes. $n \geq 7$ animals per condition. $\mathrm{N} \geq 5$ plaques per animal. Unpaired student's t-test. ${ }^{*} \mathrm{p}<0.05,{ }^{* *} \mathrm{p} \leq 0.01$. 


\subsubsection{No differences in concentrations of soluble and insoluble $A \beta$-peptides were found in $\mathrm{Rab}^{\triangle \mathrm{MG}} \times 5 \times \mathrm{xAD}$}

We next examined soluble and insoluble $A \beta_{38}, A \beta_{40}$ and $A \beta_{42}$ concentrations in the hippocampus of 3 month and 9 month old animals.

In order to analyze this, different soluble $A \beta$-fractions from snap-frozen hippocampal tissue were isolated (illustrated in Figure 19). Therefore a well-established protocol for sequential $A \beta$-isolation based on extraction with RIPA buffer, SDS buffer and $70 \%$ formic acid (FA) in addition to ultracentrifugation steps, was used (Figure 19).

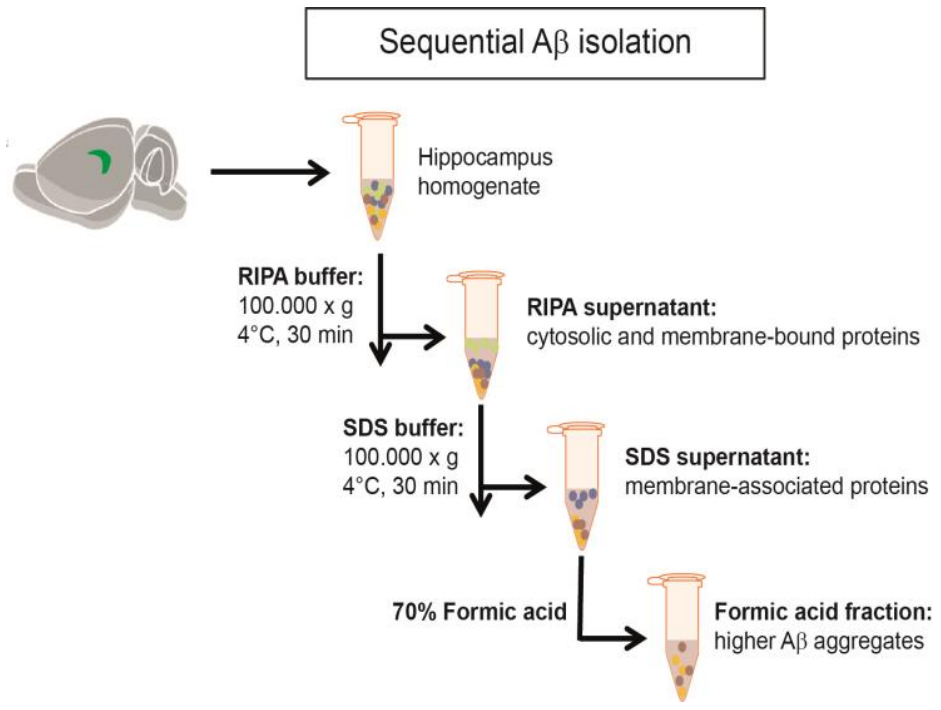

Figure 19: Workflow of sequential $A \beta$ isolation from brain homogenates.

Snap frozen hippocampi were homogenized in PBS buffer. Homogenates were mixed with RIPA buffer and after initial cell lysis centrifuged to remove cellular debris. The RIPA supernatants containing cytosolic and membrane-bound proteins were snap frozen for future analysis. RIPA insoluble pellets were further processed with SDS buffer and centrifugation obtained SDS supernatants were snap frozen. The remaining pellets were mixed with $70 \%$ formic acid and snap frozen. All samples were stored at $-80^{\circ} \mathrm{C}$ until further use.

The obtained fractions were analyzed for $A \beta_{38}, A \beta_{40}$ and $A \beta_{42}$ by MSD technology (Mesoscale, Gaithersburg, MD, USA). MSD is an electrochemiluminescence (ECL) assay which combines the high sensitivity of ECL with a sandwich enzyme-linked immunosorbent assay (ELISA). Therefore analysis of a broad detection range is possible. Due to defined physical locations $A \beta$-species specific capturing antibodies coated to the wells, simultaneous measurement of $A \beta_{38}, A \beta_{40}$ and $A \beta_{42}$ within one well is possible. Due to the high sensitivity of the assay the sequential fractions were diluted in the respective buffers. The formic acid fraction was diluted in $\mathrm{pH}$ neutral Tris buffer. To receive comparable results, the measured values were normalized to the total protein amount of the homogenate measured by BCA assay of the respective samples.

Analyzing the probes of 3 month old females, $A \beta_{38}$ was only detectable in SDS buffer extracts (Figure 20 A, E, I, M). Between Rab7 $7^{\mathrm{flfl}} \times 5 \times \mathrm{FAD}$ and Rab7 $\triangle \mathrm{MG} \times 5 \times \mathrm{FAD}$ there was no difference found in the levels of $A \beta_{38}$ normalized to total protein content (Figure $20 \mathrm{E}$ ). Also the levels of $A \beta_{40}$ and $A \beta_{42}$ did not show any differences between the genotypes within extracts in RIPA buffer (Figure 20 B, C), SDS buffer (Figure 20 F, G) and formic acid (Figure 20 I, J). Additionally, the analysis of the $A \beta_{42}$ to $A \beta_{40}$ ratio did not detect differences within the respective fractions at 3 month

$$
\text { 61| P a g e }
$$




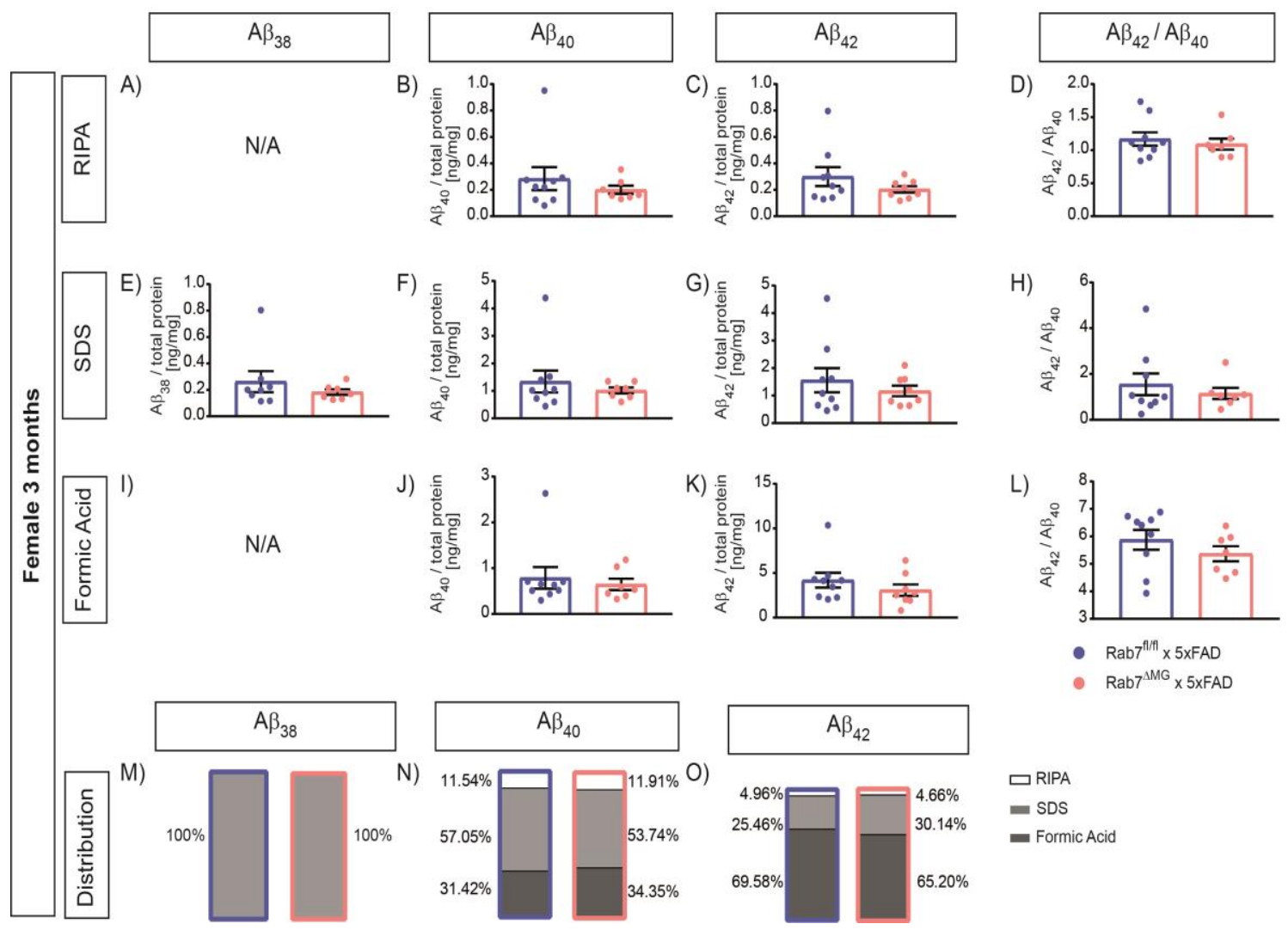

Figure 20: MSD analysis of 3 month old females did not show differences in the aggregation states of $A \beta_{38}, A \beta_{40}$ and $A \beta_{42}$.

A-C) Amounts of $A \beta_{38}, A \beta_{40}$ and $A \beta_{42}$ normalized to total protein concentration [ng/mg] within the RIPA fraction are illustrated. D) The $A \beta_{42} / A \beta_{40}$ ratio in the RIPA fraction is shown. E-H) Graphs of the amounts of $A \beta_{38}, A \beta_{40}$ and $A \beta_{42}$ as well as the $A \beta_{42} / A \beta_{40}$ ratio in the SDS extracts are shown. I-L) The respective measurements in the formic acid fractions of 3 month old females are presented. A+l) $A \beta_{38}$ was not detectable in the RIPA and formic acid fraction, indicated by N/A (not applicable). Graphs are presented as mean \pm SEM with the individal samples added to the blot as circles. M-O) Ratio [\%] of $A \beta_{38}, A \beta_{40}$ and $A \beta_{42}$ in the respective, measured fractions are shown. The percentage within the RIPA fraction is shown in white, the amount in the SDS fraction is colored in light grey and the ratio in formic acid is illustrated in dark grey with the precise values written next to the bars. The bars framed in blue show the measurements of Rab7 $7^{\text {fl/1/1}} \times 5 \times F A D$ females, the bars in pink represent the Rab7 ${ }^{\triangle M G} \times 5 \times F A D$ animals. $n \geq 8$ animals. Measurements in duplicates.

old animals (Figure 20 D, H, K). Finally, we addressed the question whether Rab7 ${ }^{\triangle M G} \times 5 \times F A D$ females showed alterations within the aggregation status of $A \beta_{40}$ and $A \beta_{42}$. Therefore the relative concentrations of the analyzed $A \beta$-peptides within the single solvents were set into relation to the overall amount of $A \beta$-peptide (Figure $20 \mathrm{~L}-\mathrm{N}$ ). In conclusion, we could not detect any differences in the levels and aggregation states of $A \beta_{38}, A \beta_{40}$ and $A \beta_{42}$ in the hippocampus at 3 month old

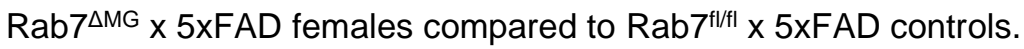

To elucidate whether the deposition of $A \beta_{38}, A \beta_{40}$ and $A \beta_{42}$ had changed throughout disease progression, MSD measurements from fractions of hippocampal tissue of 9 month old Rab7 ${ }^{\Delta M G} \mathrm{X}$ 5xFAD females were analyzed, too (Figure 21). In contrary to the samples at 3 months by 9 
months $A \beta_{38}$ was also present in the RIPA buffer extracts (Figure $21 \mathrm{~A}$ ). In RIPA and SDS fractions there were no differences between the two conditions though in the levels of $A \beta_{38}$ (Figure 21 A, E). Also the levels of $A \beta_{40}$ and $A \beta_{42}$ showed no differences between the conditions in none of the three extracts (Figure $21 \mathbf{B}, \mathbf{C} ; \mathbf{F}, \mathbf{G}$; I, J). The $A \beta_{42} / A \beta_{40}$ ratio did also not differ between $R a b 7^{f / f l} x$ $5 \times F A D$ and Rab7 ${ }^{\triangle M G} \times 5 \times F A D$ females at 9 months of age. The distribution of $A \beta_{38}, A \beta_{40}$ and $A \beta_{42}$ within the three fractions did not show alterations either (Figure 21 L-N).

Therefore we can conclude that there were no differences in soluble and insoluble $A \beta_{38}, A \beta_{40}$ and $A \beta_{42}$ between $R a b 7^{f / 1 / 1} \times 5 \times F A D$ and Rab7 ${ }^{\Delta M G} \times 5 \times F A D$ females at 9 months.

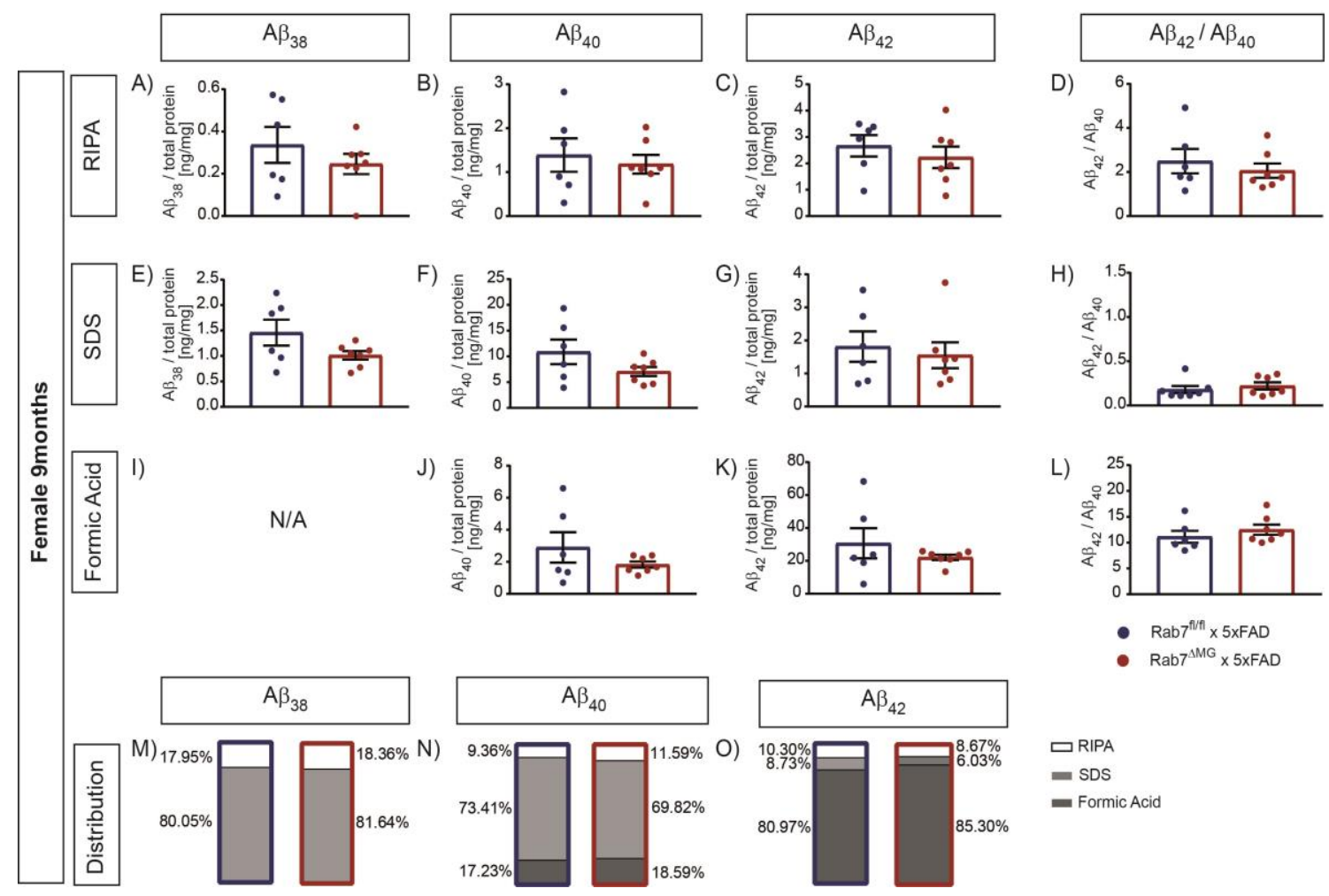

Figure 21: The amounts of soluble and insoluble $A \beta_{38}, A \beta_{40}$ and $A \beta_{42}$ in 9 month old Rab7 ${ }^{\Delta M G} \times 5 \times F D$ females were not changed.

A-D) Amounts of $A \beta_{38}, A \beta_{40}$ and $A \beta_{42}$ normalized to the total protein concentration [ng/mg] and the ratio of $A \beta_{42} / A \beta_{40}$ in the RIPA fractions are shown. E-H) Identical measurements of the SDS fractions are presented. I) No $A \beta_{38}$ was detectable in the formic acid fraction (N/A). J-L) Measurements of $A \beta_{40}, A \beta_{42}$ and the $A \beta_{42} / A \beta_{40}$ ratio within the formic acid fractions in 9 month old females are illustrated. Graphs are shown as mean \pm SEM with the individal measurements added to the blot as circles. $M-0$ ) Distribution [\%] of $A \beta_{38}, A \beta_{40}$ and $A \beta_{42}$ within the RIPA fraction (white), the SDS fraction (light grey) and the fromic acid fraction (dark grey) are shown. The exact values are next to the graphs. The bars in dark blue frames show Rab7 $7^{\mathrm{fl} / \mathrm{fl}} \times 5 \times \mathrm{FAD}$ females, the bars in red frames present Rab7 $7^{\triangle M G} \times 5 \times F A D$ females at 9 months. $\mathrm{n} \geq 6$ animals. Measurements in duplicates. 
The male cohorts were analyzed likewise. For 3 month old Rab7 ${ }^{\triangle M G} \times 5 \times F A D$ males, we also could not detect differences to their Rab7 f//fl $\times 5 \times F A D$ litter mates in the protein levels of $A \beta_{38}, A \beta_{40}$ and $A \beta_{42}$ in the RIPA buffer fraction (Figure 22 A-C), the SDS buffer fraction (Figure 22 E-G) and in the formic acid fraction (Figure 22 I-K), respectively. Also the $A \beta_{42}$ to $A \beta_{40}$ ratios within all three solvents were the same for Rab7 $7^{\mathrm{flfl}} \times 5 \times \mathrm{FAD}$ and Rab7 ${ }^{\triangle \mathrm{MG}} \times 5 \times \mathrm{FAD}$ males (Figure $22 \mathrm{D}, \mathbf{H}, \mathbf{L}$ ). The distributions of $A \beta_{40}$ and $A \beta_{42}$ within the three fractions showed no changes between Rab7 fl/fl $x$ 5xFAD and Rab7 $\triangle M G$ x 5xFAD males (Figure $22 \mathrm{~N}, \mathbf{O}$ ). $A \beta_{38}$ was exclusively found in the SDS fraction (Figure 22 A, E, I, M).

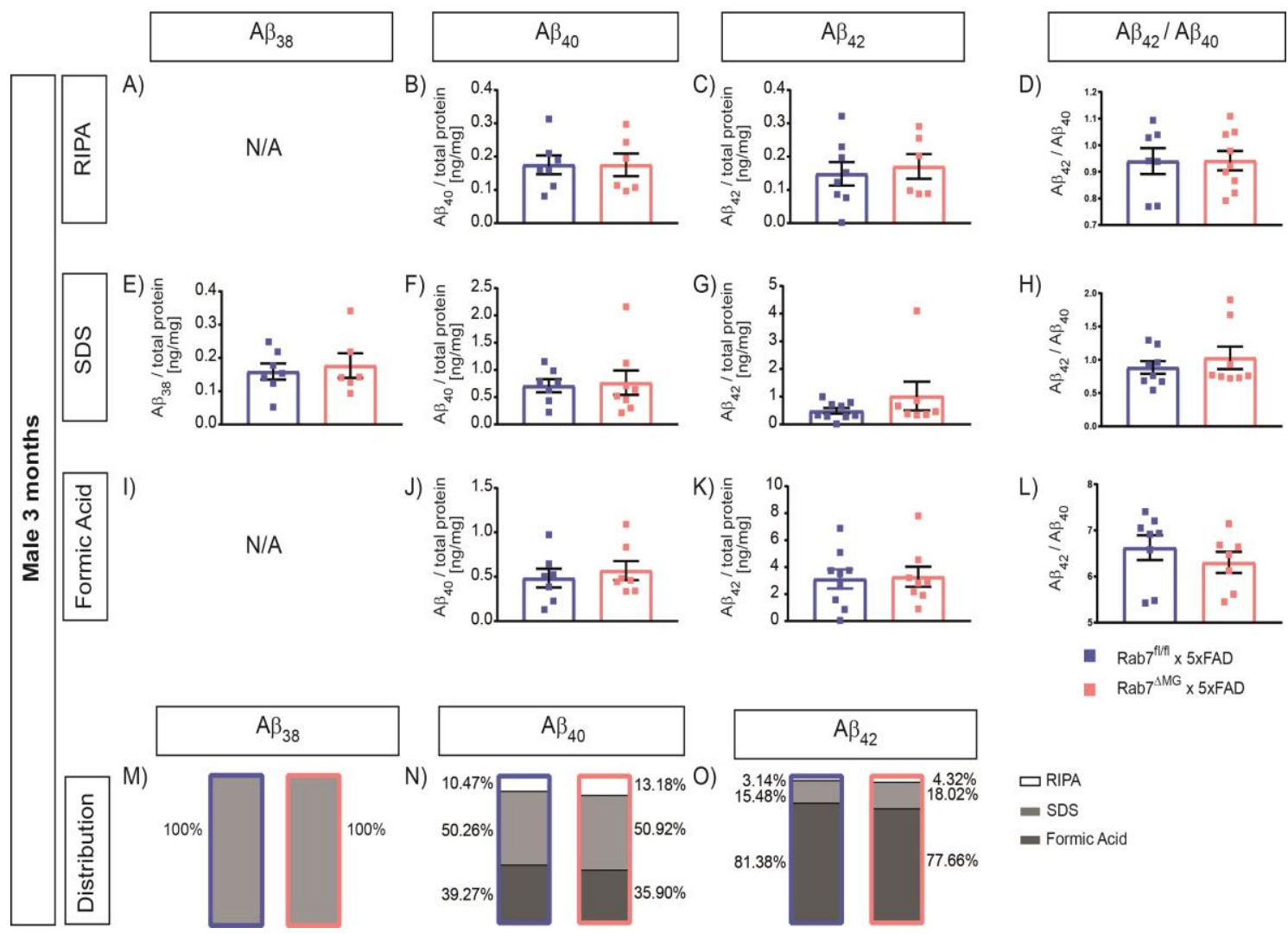

Figure 22: No differences in the aggregation states of $A \beta_{38}, A \beta_{40}$ and $A \beta_{42}$ were found in 3 month old Rab7 $7^{\Delta M G} x$ 5xFAD males.

A-D) Amounts of $A \beta_{38}, A \beta_{40}$ and $A \beta_{42}$ as well as of $A \beta_{42} / A \beta_{40}$ in the RIPA extracts were determined by MSD. E-H) Measurements of $A \beta_{38}, A \beta_{40}$ and $A \beta_{42}$ and of $A \beta_{42} / A \beta_{40}$ in SDS fractions are shown. J-L) Quantification of $A \beta_{40}$, $A \beta_{42}$ and the $A \beta_{42} / A \beta_{40}$ ratio in the formic acid fractions are presented. $\left.A+I\right) A \beta_{38}$ was not detectable in the RIPA and the formic acid fractions (N/A). Graphs are shown as mean \pm SEM with the individal measurements added as squares to the graphs. M-O) Fractions [\%] of $A \beta_{38}, A \beta_{40}$ and $A \beta_{42}$ within the RIPA fraction (white), the SDS fraction (light grey) and the fromic acid fraction (dark grey) are presented. The calculated values are written next to the graphs. The graphs in blue frames represent 3 month old Rab7 $7^{\text {fl/fl }} \times 5 \times$ FAD males and the bars in pink frames show the Rab7 $7^{\triangle M G} \times 5 \times F A D$ males. $n \geq 7$ animals. Measurements in duplicates. If values were below detection range, they were excluded from the graphs. 
Finally, the MSD measurements of soluble and insoluble $A \beta$ obtained from hippocampi of the 9 month old males were analyzed the same way. In agreement with the previous data, no differences were detected in the absolute concentrations and the distributions of $A \beta_{38}, A \beta_{40}$ and $A \beta_{42}$ (Figure 23). Likewise to the female cohorts, we did not detect differences in male Rab7/f/fl $\times 5 \times F A D$ and Rab7 ${ }^{\triangle M G} \times 5 \times F A D$ mice, neither at 3 nor at 9 months within the different soluble fractions of $A \beta_{38}$, $A \beta_{40}$ and $A \beta_{42}$.

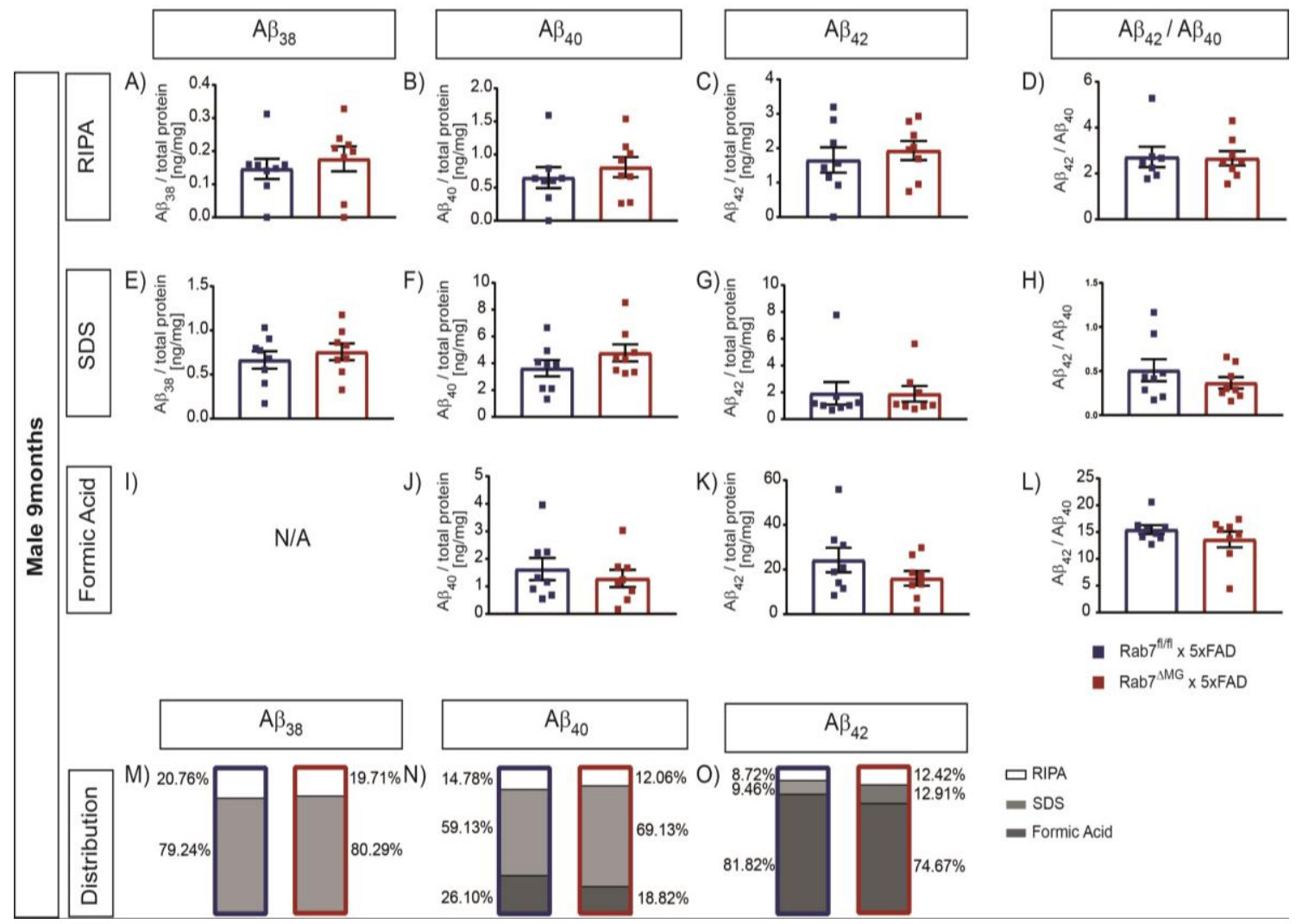

Figure 23: Aggregation states of $A \beta_{38}, A \beta_{40}$ and $A \beta_{42}$ were not changed in 9 month old Rab7 ${ }^{\triangle M G} \times 5 \times F A D$ males.

A-D) RIPA fraction analyses for $A \beta_{38}, A \beta_{40}, A \beta_{42}$ and $A \beta_{42}$ / $A \beta_{40}$ are displayed. E-H) Simultaneous measurements of the SDS fractions. I) In the formic acid fraction $A \beta_{38}$ was not detectable (N/A). J-L) Quantification of $A \beta_{40}, A \beta_{42}$ and the $A \beta_{42}$ / $A \beta_{40}$ ratio in the formic acid fractions. Graphs are shown as mean \pm SEM with the individal measurements added as squares to the graphs. M-O) Proportions [\%] of $A \beta_{38}, A \beta_{40}$ and $A \beta_{42}$ within the RIPA fraction (white), the SDS fraction (light grey) and the fromic acid fraction (dark grey) are presented. The calculated values are written beside the graphs. Bars with dark blue frames show $\operatorname{Rab}^{\mathrm{fl} / \mathrm{fl}} \times 5 \mathrm{xFAD}$ males and bars with red frames present Rab7 ${ }^{\triangle \mathrm{MG}} \times 5 \times \mathrm{FAD}$ males at 9 months. $\mathrm{n} \geq 8$ animals. Measurements in duplicates.

In summary, MSD analysis did not identify differences in the solubility of $A \beta_{38}, A \beta_{40}$ and $A \beta_{42}$ between Rab7 $7^{\mathrm{fl} / \mathrm{fl}} \times 5 \times \mathrm{FAD}$ and $\mathrm{Rab} 7^{\triangle \mathrm{MG}} \times 5 \times \mathrm{xFD}$ neither in females nor in males at 3 and 9 months, respectively. Also the ratios of $A \beta_{42} / A \beta_{40}$ were widely unchanged. Therefore we could conclude that $A \beta$-processing per se was not affected by the Rab7 ${ }^{\triangle M G} \times 5 \times F A D$. 


\subsection{Neuronal, dendritic spine and synaptic loss were not altered in the Rab7 ${ }^{\triangle M G}$ x 5 XFAD mice}

One of the most prominent hallmarks of $A D$ is neuronal loss (Alzheimer et al., 1995). Other forms of neuronal damage occur along $A D$ progression. For instance loss of synapses and dendritic spines are well reported throughout disease (Jacobsen et al., 2006; Lue et al., 1999; Mucke et al., 2000). Therefore we wanted to elucidate whether Rab7 ${ }^{\triangle M G} \times 5 \times F A D$ animals differ from Rab7 $7^{f / f 1} \times 5 \times F A D$ controls in respect to synaptic density and neuronal loss.

\subsubsection{Neuronal loss was not reduced in Rab7 ${ }^{\triangle M G} \times 5 \times F A D$ mice}

To address whether Rab7 KO in microglia increased neuronal loss in our Rab7 ${ }^{\triangle M G} \times 5 \times F A D$ mouse model, immunohistochemistry for NeuN (neuronal nuclear antigen) was done (Figure 24). By 9 months of age 5xFAD mice reveal a significant loss of neurons in cortical layer $\mathrm{V}$ and in the subiculum (Oakley et al., 2006). Therefore, Rab7 ${ }^{M M G} \times 5 \times F A D$ mice and their respective controls were analyzed in these two brain regions for neuronal loss. Comparing 3 month to 9 month old mice, neuronal loss was clearly visible in the older animals for both sexes (Figure 24 A-LFigure 24 O-Z).

By 3 months females showed no differences in NeuN+ cells / area between Rab7 fl/fl $x$ 5xFAD and Rab7 ${ }^{\triangle M G}$ x 5xFAD in cortex (Figure 24 C, E, M) or subiculum (Figure 24 D, F, N). Cell counts are moderately reduced in the cortex (Figure $24 \mathbf{~ I , ~ K , ~ M ) ~ a n d ~ s t r o n g l y ~ d i m i n i s h e d ~ i n ~ t h e ~ s u b i c u l u m ~}$ (Figure $24 \mathrm{~J}, \mathrm{~L}, \mathbf{N}) 6$ months later. Even though Rab7 $7^{\mathrm{fl} / \mathrm{fl}} \times 5 \times \mathrm{FAD}$ and Rab7 ${ }^{\triangle \mathrm{MG}} \times 5 \times \mathrm{FAD}$ females showed no difference in the cortex, there was a trend towards less neuronal loss in the subiculum of Rab7 $\triangle M G$ x 5xFAD females, though.

For male animals of the Rab7 ${ }^{\triangle M G} \times 5 \times F A D$ mouse line there was no difference of NeuN+cells /area detectable within the cortex (Figure 24 Q, S, A') or within the subiculum (Figure 24 R, T, B') by 3 months of age. Within the subiculum neuronal loss was more prominent (Figure $24 \mathbf{X}, \mathbf{Z}, \mathbf{B}$ '). However, no difference was measured between the Rab7 $7^{\mathrm{fl} / \mathrm{fl}} \times 5 \mathrm{xFAD}$ and the Rab7 $\triangle \mathrm{MG} \times 5 \times \mathrm{xFD}$ mice at 9 months in these two regions (Figure $24 \mathbf{W}, \mathbf{Y}, \mathbf{A}^{\prime}$ ).

Thus, we can conclude that Rab7 ${ }^{\triangle M G} \times 5 \times F A D$ has no impact of neuronal loss neither in males nor females. 


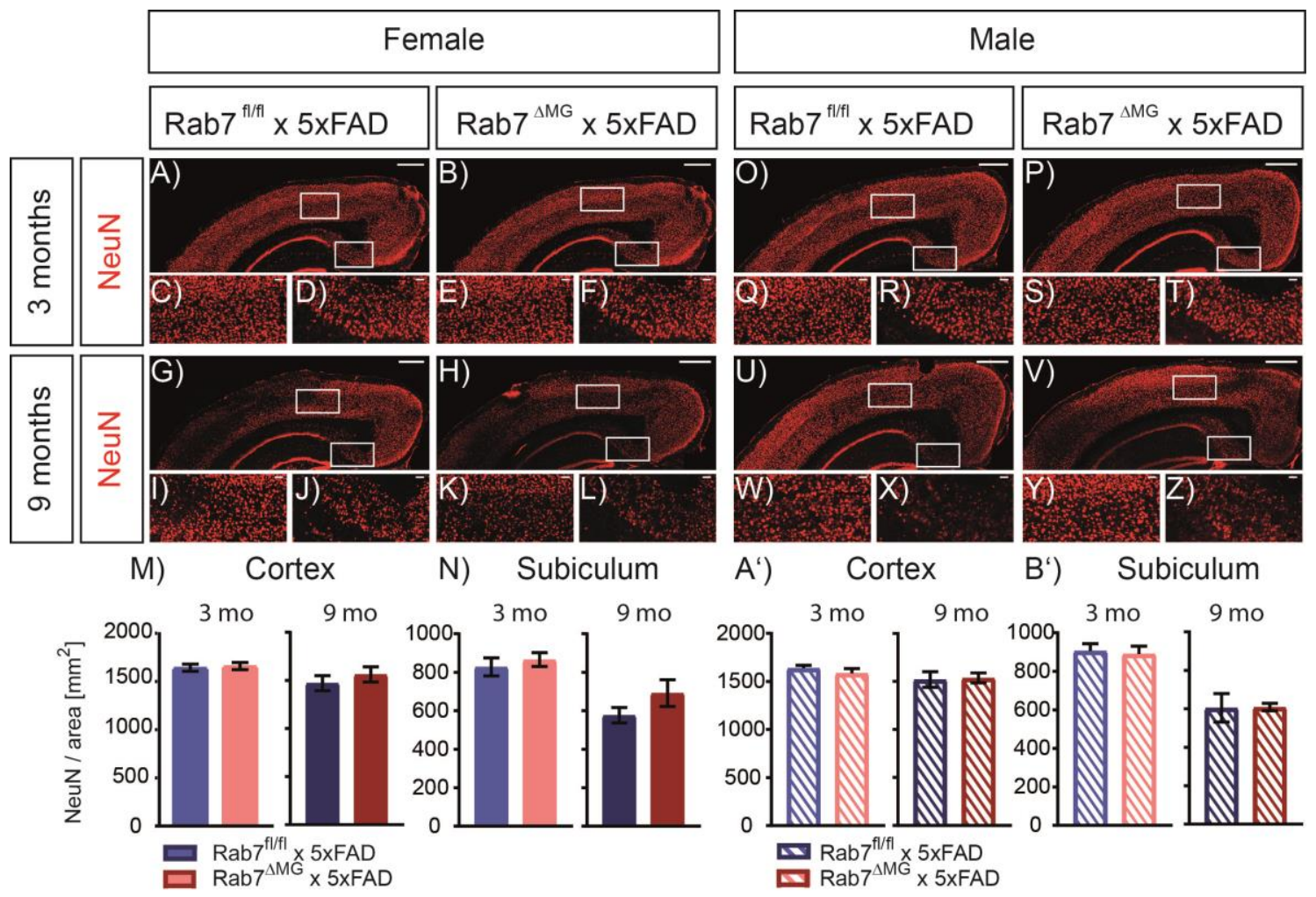

Figure 24: Rab7 ${ }^{\triangle M G}$ x 5xFAD mice did not decelerate neuronal loss.

A, B, O, P) Maximum intensity projections of confocal images of 3 month old animals stained for NeuN (red) are shown. G, H, U, V) Representative images of NeuN stainings in 9 month old Rab7 $7^{\mathrm{fl/fl}} \times 5 \times \mathrm{FAD}$ and Rab7 $7^{\triangle \mathrm{MG}} \times 5 \times \mathrm{xAD}$ animals are shown. $\mathbf{C}, \mathbf{E}, \mathbf{I}, \mathbf{K}, \mathbf{Q}, \mathbf{S}, \mathbf{W}, \mathbf{Y}$ ) Close-ups within the cortex and $\mathbf{D}, \mathbf{F}, \mathbf{J}, \mathbf{L}, \mathbf{R}, \mathbf{T}, \mathbf{X}, \mathbf{Z}$ ) within the subiculum are shown underneath the respective tile scan. Magnified areas within the macroscopic images are labeled with white rectangles. Scale bar of macroscopic images: $500 \mu \mathrm{m}$; scale bar of the close-ups: $500 \mu \mathrm{m}$. M, A') Neuronal loss quantified in the cortex and $\mathbf{N}, \mathbf{B}^{\prime}$ ) in the subiculum of 3 and 9 month old Rab7 $7^{\text {tl/fl }} \times 5 \times F A D$ (blue/ dark blue) and $\mathrm{Rab}^{\triangle \mathrm{MG}} \times 5 \times \mathrm{XFD}$ (pink/red) females (filled bars) and males (striped bars), respectively. $\mathrm{N} \geq 3$ sections for cortex and $\mathrm{N}=2$ sections for subiculum per animal. $\mathrm{n}=7$ females, $\mathrm{n}=8$ males. Data are presented as mean $\pm \mathrm{SEM}$.

\subsubsection{Rab7 ${ }^{\triangle M G} \times 5 \times F A D$ showed mild effects on dendritic spine loss}

Another prominent feature of $A D$ is the loss of dendritic spines. In the 5xFAD mouse model dendritic spines are significantly reduced in 14 month old mice compared to WT controls (Spangenberg et al., 2016).

To investigate whether Rab7 ${ }^{\triangle M G} \times 5 \times F A D$ showed alterations in this pathological feature, Golgi-Cox analysis was done with tissue of 9 month old animals (Figure 25 A, B, E, F). The overall numbers of dendritic spines were not significantly altered in females and males due to the Rab7 KO (Figure $25 \mathbf{C}+\mathbf{G})$. However, when the specific spine forms were analyzed, Rab7 $\triangle M G$ x $5 \times$ FAD females showed significantly more mushroom spines (Figure $25 \mathrm{D}$ ), whereas males had no detectable differences within dendritic spine classes (Figure $25 \mathrm{H}$ ). 

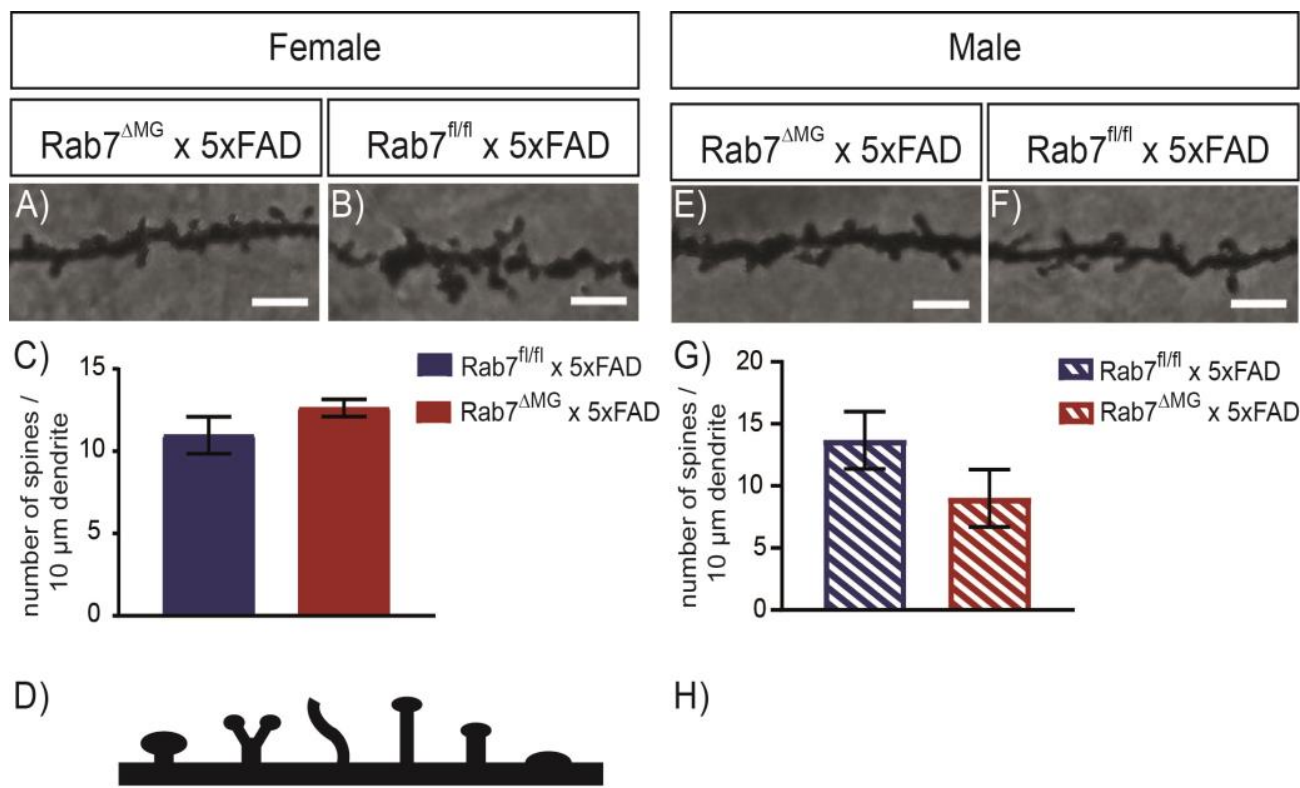

H)
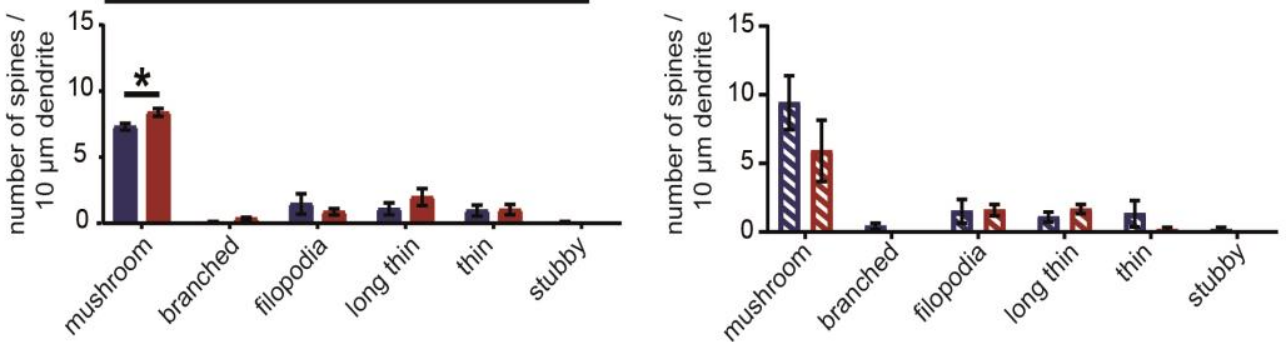

Figure 25: Mushroom spine loss was reduced in Rab7 ${ }^{\Delta M G}$ x 5xFAD females.

A, B, E, F) Dendrites were impregnated with mercury containing Golgi-Cox solution to visalize neuronal morphology. Brain sections of 9 month old females and males of the Rab7 ${ }^{\triangle M G} \times 5 \times F A D$ mouse line were imaged within cortical layers IV-VI with 100x magnification. Secondary apical dendrites were chosen for analysis. Here, z-stack projections are shown. Scale bar: $5 \mu \mathrm{m}$. C, G) Quantification of absolute dendritic spine numbers per $10 \mu \mathrm{m}$ dendrites were analyzed from female and male samples. D) Classification of the quantified dendritic spines depending on ratio of spine length and thickness is illustrated. D, H) Quantification according to dendritic spine types is shown of Rab7 $7^{f / f l} \times 5 \times F A D$ and Rab7 ${ }^{\triangle M G} \times 5 \times F A D$ females and males. Data are shown as mean \pm SEM. $n=3$ animals per condition, $N=5$ dendrites per animal. Unpaired student's t-test. ${ }^{*} p \leq 0.05$. Analysis was done according to Risher et al. (2014). Quantification was done by Michaela Limmer.

\subsubsection{Synaptic loss was not altered due to Rab7 KO}

Loss of synapses is another hallmark of AD. Therefore, Western blot analysis for synaptic protein Synaptophysin was done to check for alterations within in Rab7 $\triangle M G$ x $5 \times$ FAD mice compared to Rab7 $7^{f / 1 / 1} \times 5 \times F A D$ controls. There was no difference detectable in levels of Synaptophysin between $\mathrm{Rab7}^{\mathrm{fl} / \mathrm{fl}} \times 5 \times \mathrm{xFA}$ and Rab7 $\triangle \mathrm{MG} \times 5 \times \mathrm{FAD}$ neither in females (Figure $26 \mathrm{~A}$ ) nor in males (Figure 26 B) at 9 months of age. Therefore we concluded that the Rab7 ${ }^{\triangle M G}$ did not reduce synaptic loss in the 5xFAD mouse model. 

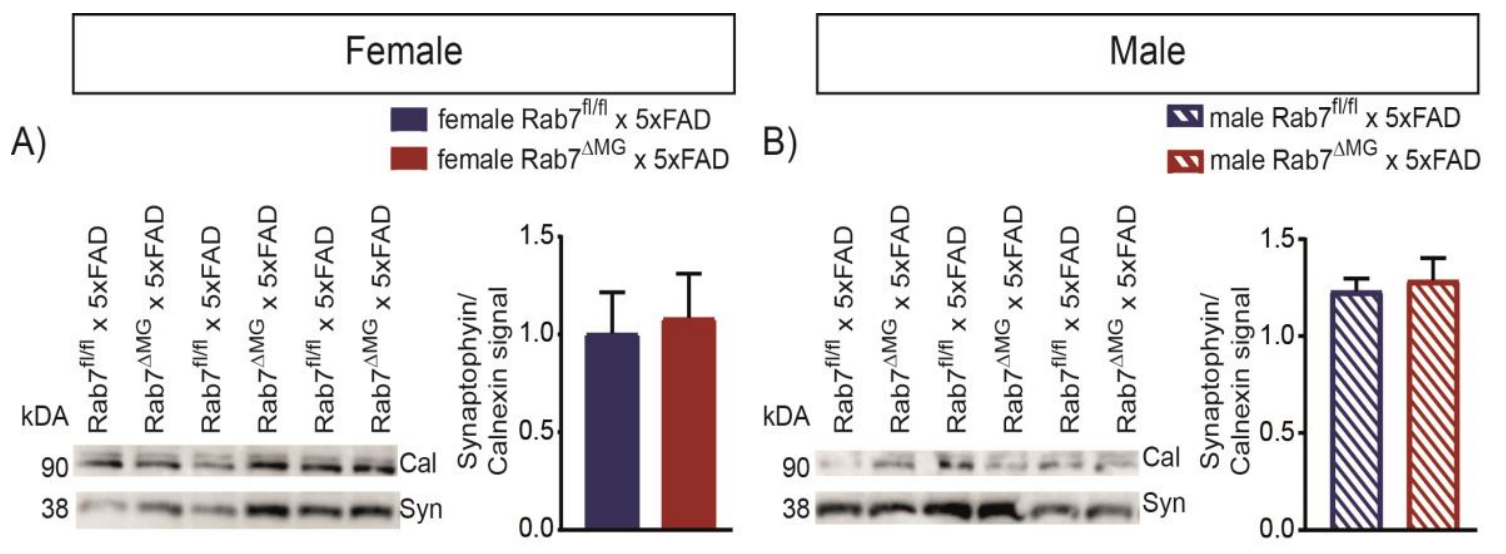

Figure 26: Synaptic loss was not decelerated by Rab7 ${ }^{\Delta M G}$ in 5xFAD mice.

A) Prepresentative Western blots probed for Synaptophysin (Syn) and Calnexin (Cal) as housekeeper on 3 individual samples of 9 month old Rab7 $7^{\text {fl/fl }} \times 5 \times F A D$ and Rab7 ${ }^{\triangle M G} \times 5 \times F A D$ females. Quantification of Synatophysin normalized to the Calnexin signal of Rab7 $7^{f / 1 / 1} \times 5 \times F A D$ (blue) and Rab7 $7^{\triangle M G} \times 5 \times F A D$ (red) females. B) Western blots and quantifications of Syn and Cal on samples at 9 month old Rab7//fl $\times 5 \times F A D$ (blue striped) and Rab7 $7^{\triangle M G} \times 5 \times F A D$ (red striped) males. $\mathrm{n} \geq 8$ animals. Data are presented as mean \pm SEM.

\subsection{Phenotypical characterization of microglia in the Rab7 $^{\triangle M G}$ x 5XFAD model}

After validation of the microglial Rab7 KO in 5xFAD mice, I next addressed the question whether the Rab7 KO per se had an effect on microglia numbers, recruitment to A $\beta$-plaques and morphology. In addition, we analyzed whether Rab7 $\triangle \mathrm{MG}$ x $5 \times \mathrm{FAD}$ resulted in differential protein profiles in the brain of 5xFAD mice. To further investigate whether these features changed throughout disease progression, animals at 3 months and at 9 months of age were analyzed.

\subsubsection{Rab7 ${ }^{\triangle M G} \times 5 x F A D$ had no effects on absolute microglia numbers or on their recruitment}

Since the cortex and hippocampus are brain regions affected early in the 5xFAD mouse model by A $\beta$-pathology, these regions were analyzed at 3 and 9 months of age by immunohistochemistry (Figure 27 and Figure 28) (Oakley et al., 2006).

The absolute number of lba1+ cells / area did not show differences in the cortex and the hippocampus in females and males at 3 months, respectively (Figure $27 \mathbf{A}-\mathbf{J}$; M-V). To further elucidate whether the Rab7 ${ }^{\triangle M G}$ had an impact on the recruitment of microglia around $A \beta$-plaques, the number of $A \beta$-plaque-associated microglia (PAM) / $A \beta$-plaque was quantified. We defined PAM as all lba1+ cells within a $50 \mu \mathrm{m}$ radius of a $6 \mathrm{E} 10+\mathrm{A} \beta$-plaque. Since $6 \mathrm{E} 10$ is an antibody recognizing amino acids 3-8 within the $A \beta$-peptide sequence and therefore also unprocessed, 
intracellular APP in neurons, extracellular A $\mathrm{A}$-plaques were selected by morphology. There was no difference in the number of Iba1+PAM cells / A 3 -plaque in the cortex or the hippocampus at 3 months of age in neither females nor males (Figure 27 K, L, W, X).

By 9 months $A \beta$-plaques can be found throughout most regions of the brain of the $5 x F A D$ mouse model. Thus, we investigated whether the number of Iba1+ microglia has changed throughout the progression of the disease. There was no significant difference detectable in Iba1+ cell numbers / area in the cortex or the hippocampus of females or males (Figure $28 \mathbf{A}-\mathbf{J} ; \mathbf{M}-\mathbf{V}$ ). Interestingly though, the numbers of lba1+ cells / area were higher in females at 9 months compared to those at 3 months. This is true for Rab7 $7^{\mathrm{fl} / \mathrm{l}} \times 5 \times \mathrm{XAD}$ and Rab7 ${ }^{\triangle M G} \times 5 \times F A D$ females for both investigated brain regions (Figure $27 \mathrm{I}$, J; Figure $28 \mathrm{I}, \mathbf{J}$ ). There was no difference in Iba1+ cell numbers in male animals (Figure $27 \mathrm{U}, \mathbf{V}$; Figure $28 \mathrm{U}, \mathbf{V}$ ). Additionally, there was no significant difference in the number of Iba1+PAM / A $\beta$-plaque at 9 months in female and male animals, respectively (Figure 28 $K, L, W, X)$. 

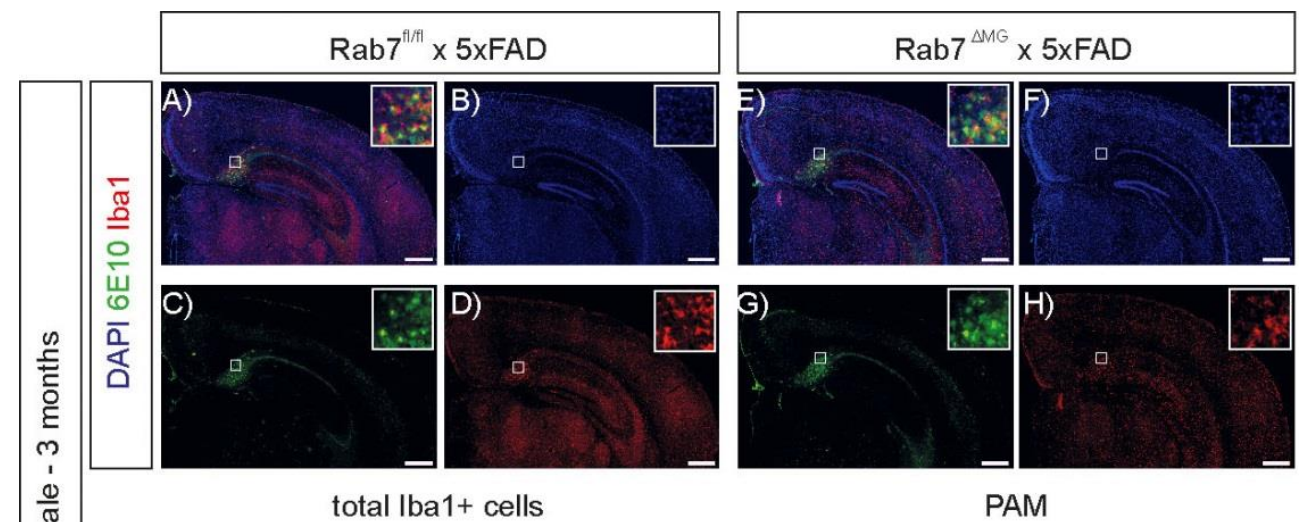

I)

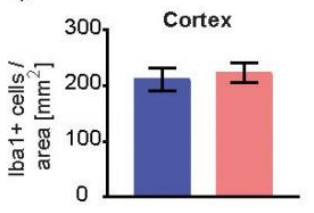

J)

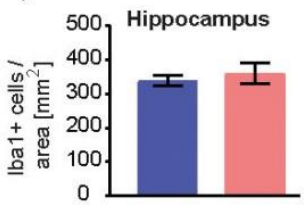

K)

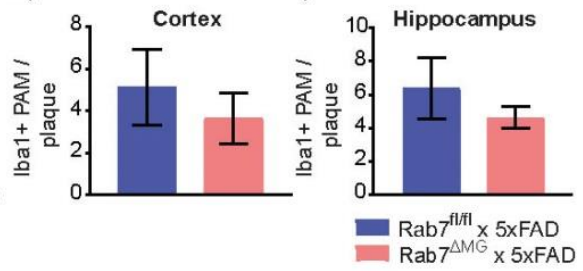

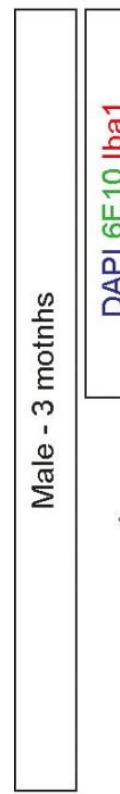
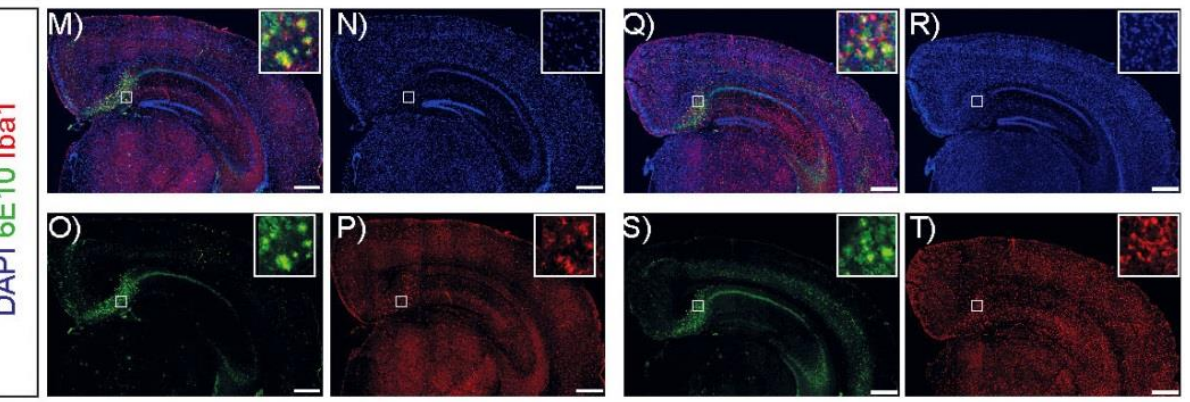

total Iba1+ cells

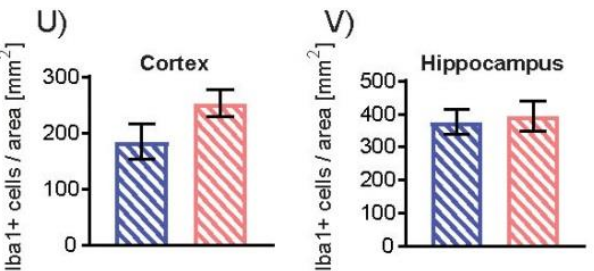

W)

PAM

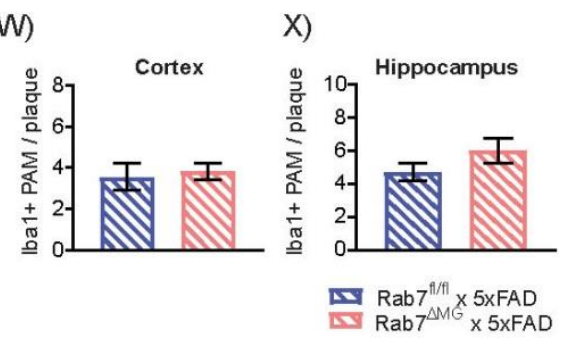

Figure 27: Cell numbers and recruitment of Iba1+ microglia to $A \beta$-plaques were not affected in 3 month Rab7 $^{\Delta M G}$ x 5xFAD mice.

A-H) Immunohistochemical stainings on brain sections (cortex and hippocampus) of 3 month old Rab7//fl $\times 5 \times F A D$ and Rab7 ${ }^{\triangle M G} \times 5 \times F A D$ females. A+E) Overlay images of the co-stainings for B+F) DAPI (blue), $\left.\mathbf{C}+\mathbf{G}\right) 6 \mathrm{E} 10$ (green) and D+H) Iba1 (red). Magnifications within the A $\beta$-plaque rich subiculum are highlighted with a white box and projected in the right upper corner. Scale bar: $500 \mu \mathrm{m}$. I) Numbers of lba1+ cells/ area [mm²] in the cortex and J) in the hippocampus were quantified for Rab7 $7^{\text {fl/fi }} \times 5 \times F A D$ (blue) and Rab7 $7^{\triangle M G} \times 5 x F A D$ (pink) females. $K+L$ ) Count of the numbers of plaque-assoicated microglia (PAM) per A $\mathrm{B}$-plaque are presented. $\mathbf{M}-\mathbf{T}$ ) Likewise, immunohistochemistry for DAPI (blue), $6 \mathrm{E} 10$ (green) and lba1 (red) are shown on sections of 3 month old Rab7 $7^{\mathrm{fl} / \mathrm{fl}} \times 5 \times \mathrm{xFD}$ and Rab7 ${ }^{\triangle \mathrm{MG}} \times 5 \times \mathrm{FAD}$ males. Scale bar: $500 \mu \mathrm{m}$. Quantifiations of lba1+ cells/ area $\left[\mathrm{mm}^{2}\right] \mathrm{U}$ ) in the cortex and V) in the hippocampus for $\operatorname{Rab}^{7 / / 1 / 1} \times 5 \times F A D$ (blue stripes) and Rab7 ${ }^{\triangle M G} \times 5 \times F A D$ (pink stripes) males are shown. $W_{+} X$ ) Numbers of PAM per A plaque in the cortex and hippocampus are shown for the male cohort. For both sexes $n=5$ animals with $N \geq 1$ sections were analyzed. Data are presented as mean \pm SEM. Analysis was done by Michaela Limmer. 


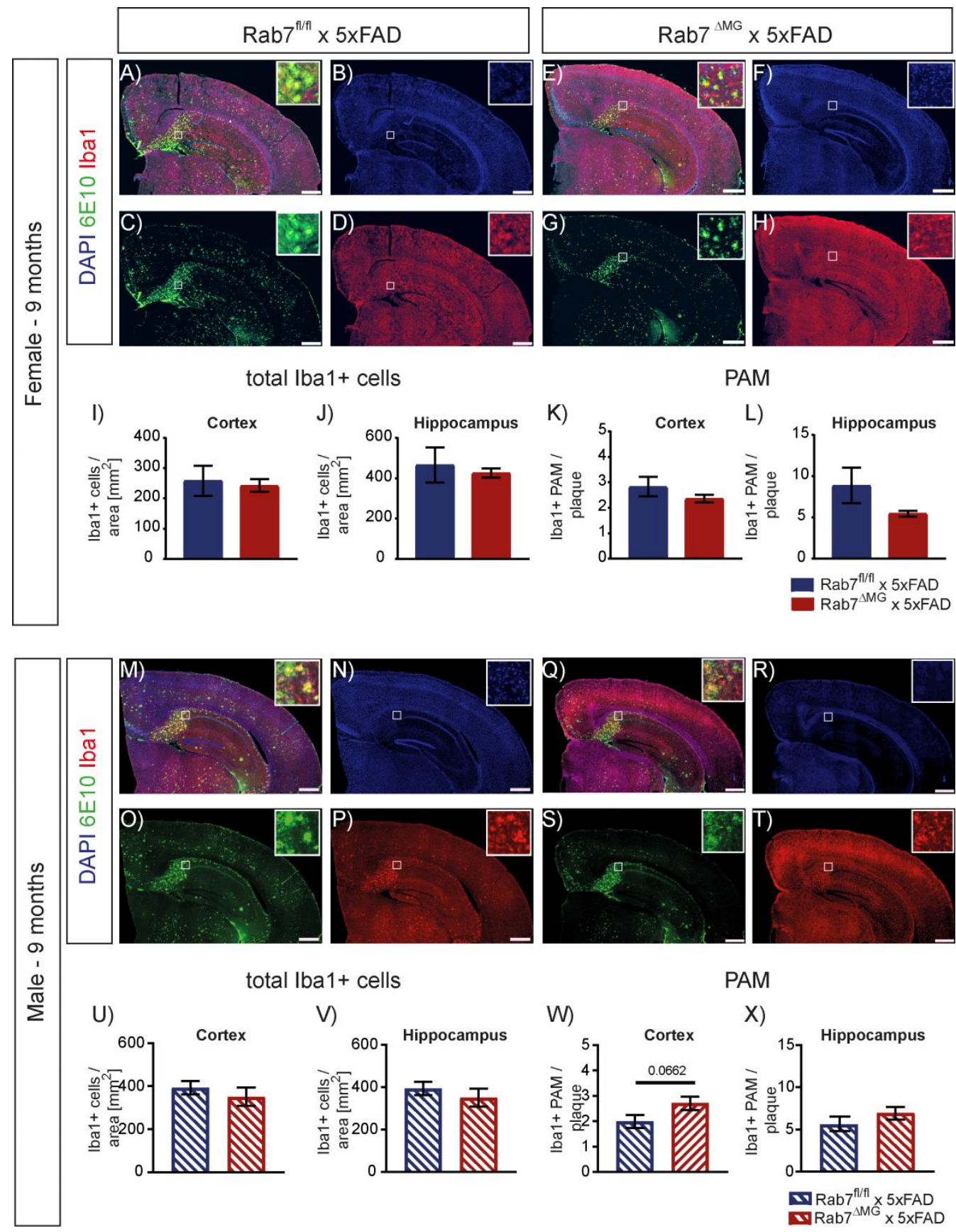

Figure 28: Cell numbers and recruitment of Iba1+ microglia to A -plaques were not impaired in 9 month old $\mathrm{Rab7}^{\mathrm{MMG}}$ x 5XFAD mice.

A-H) Representative images show immunohistochemical stainings on brain sections of 9 month old Rab7 $7^{\mathrm{fl} / \mathrm{fl}} \times 5 \times \mathrm{FAD}$ and Rab7 ${ }^{\triangle M G} \times 5 \times F A D$ females. A+E) Merge images of the co-stainings for B+F) DAPI (blue), $\left.\mathbf{C}+\mathbf{G}\right) 6 \mathrm{E} 10$ (green) and D+H) Iba1 (red). Cutout within the subiculum are highlighted with a white box and magnified in the right upper corner. Scale bar: $500 \mu \mathrm{m}$. I) Numbers of Iba1+ cells / area $\left[\mathrm{mm}^{2}\right]$ in the cortex and J) in the hippocampus were quantified for

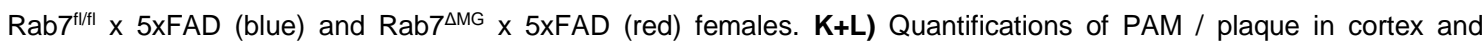
hippocampus are presented. M-T) Corresponding immunohistochemical staining on brain slices of 9 month old Rab7 $7^{\text {t/fll }} \times$ 5xFAD and Rab7 ${ }^{\triangle M G} \times$ 5xFAD males are displayed. Scale bar: $500 \mu \mathrm{m}$. U+V) Graphs show numbers of Iba1+ cells / area and $\mathbf{W + X}$ ) of PAM / plaque in the cortex and in the hippocampus for Rab7 $7^{\mathrm{fl/fl}} \times 5 \times \mathrm{FAD}$ (blue stripes) and Rab7 $7^{\Delta M G} \times$ $5 x F A D$ (red stripes) males. For both sexes $n \geq 5$ animals with $N \geq 1$ sections per animal analyzed. Data are presented as mean \pm SEM. 


\subsection{2. $R a b 7^{\triangle M G} \times 5 \times F A D$ did not display altered cellular morphology of microglia}

Since we only observed changes in A $\beta$-pathology and on mushroom spine loss in 9 month old females, we proceeded by only analyzing this cohort. As a consequence of cell activation microglia undergo amongst others changes in morphology: whereas resident microglia reveal thin processes, which are moderately ramified, activated microglia are characterized by their amoebic, round morphology. Thus the ramification index, which defines a spherical-like morphology of a cell having 1 for a perfect sphere, was calculated. Therefore 3D analysis of confocal images from lba1 staining was done (Figure 29 A-D), since lba1 is expressed throughout the cytoplasm of microglia and thereby can be used to define the cellular morphology (Imai et al., 1996; Ito et al., 1998).

To distinguish between $A \beta$-activated microglia and basal microglia activity ramification was quantified for PAM (Figure 29 A, B, E), and A -plaque distant, non-PAM microglia (Figure 29 C, D, F). This analysis showed that PAM (Figure $29 \mathrm{~B}$ ), and A -plaque distant, non-PAM microglia (Figure $29 \mathrm{C}$ ), were highly activated according to their morphological shape with an average of approximately 1 . However, there were no differences measurable between Rab7t/fli $\times 5 \times F A D$ and Rab7 ${ }^{\triangle M G} \times 5 \times F A D$ females at 9 months of age. Thus, Rab7 $\triangle M G$ $\times 5 \times F A D$ does not impair microglia activity in terms of morphological changes towards amoeboid shape.

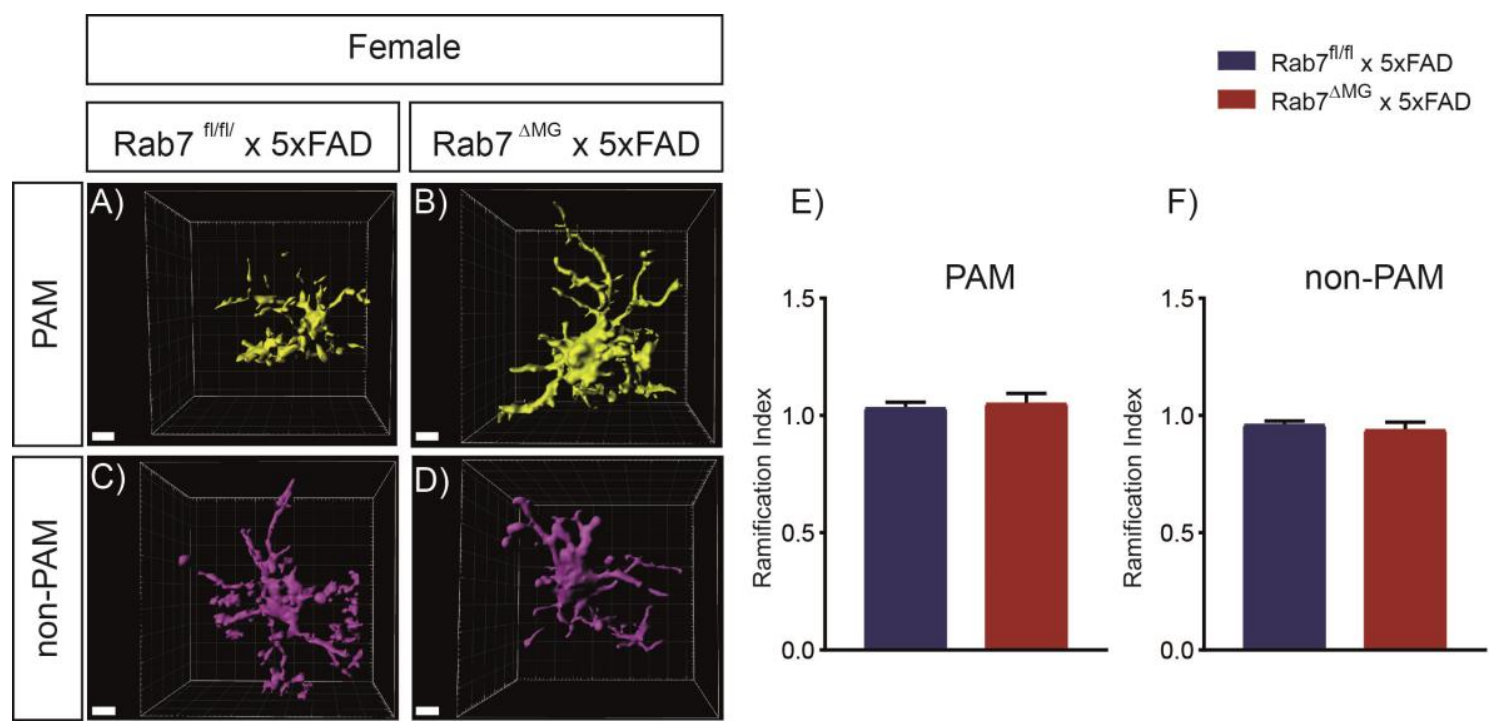

Figure 29: Morphology of Rab7 ${ }^{\Delta M G}$ x 5xFAD microglia was not altered.

A-D) 3D reconstrutions of confocal obtained z-stacks of lba1 immunohistochemical staining from the cortex. A+B) Reconstrictions of PAM (yellow) and C+D) non-PAM (purple). Scale bar: $10 \mu \mathrm{m}$. E) Ramification index of PAM was quantified. F) Ramification index of non-PAM was analyzed. The ramification index is the cell surface set into relation to the cell volume. A value of 1 represents a perfect sphere. Rab7 $7^{\mathrm{flfl}} \times 5 \times \mathrm{FAD}$ animals (dark blue) and Rab7 $7^{\triangle M G}$ $x$ xxFD animals (red) were analyzed at 9 months of age. Per condition $N=5$ cells of $n \geq 4$ animals were analyzed. Data are presented as mean \pm SEM. 


\subsection{3. $C C L 2$ was upregulated in Rab7 ${ }^{\triangle M G} \times 5 \times F A D$ mice}

The next question we addressed was how the expression pattern of different inflammation

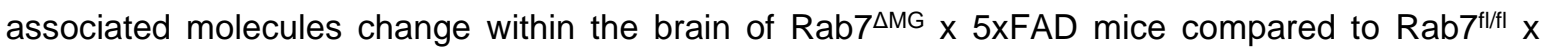
5xFAD controls. Therefore we used hippocampal RIPA-buffer extracts and send the samples for Olink® MOUSE EXPLORATORY (Olink Proteomics, Uppsala, Sweden) analysis. Olink is a proximity extension assay which uses biomarker specific oligonucleotide-coupled probes allowing for amplification by PCR. Due to the specificity of the oligonucleotides, simultaneous amplification of 92 murine biomarkers amongst others for inflammatory response can be achieved. A list of all proteins analyzed including their abbreviations can be found in the appendix "Abbreviations of proteins analyzed by Olink® proximity ligation assay".

Overall there were no significant differences found between Rab7 $7^{\triangle M G} \times 5 \times F A D$ and $R a b 7^{f / f l} \times 5 \times F A D$ females and males in both ages with the exception of CCL2 (chemokine (C-C motif) ligand 2 also known as monocyte chemoattractant protein 1 (MCP1); Figure 30 A). CCL2 was significantly upregulated in 9 month old Rab7 ${ }^{\triangle M G} \times 5 \times F A D$ females and males compared to their respective controls (Figure $\mathbf{3 0} \mathbf{B}, \mathbf{C}$ ). The absolute amount was also increased in all conditions compared to 3 month old animals. 


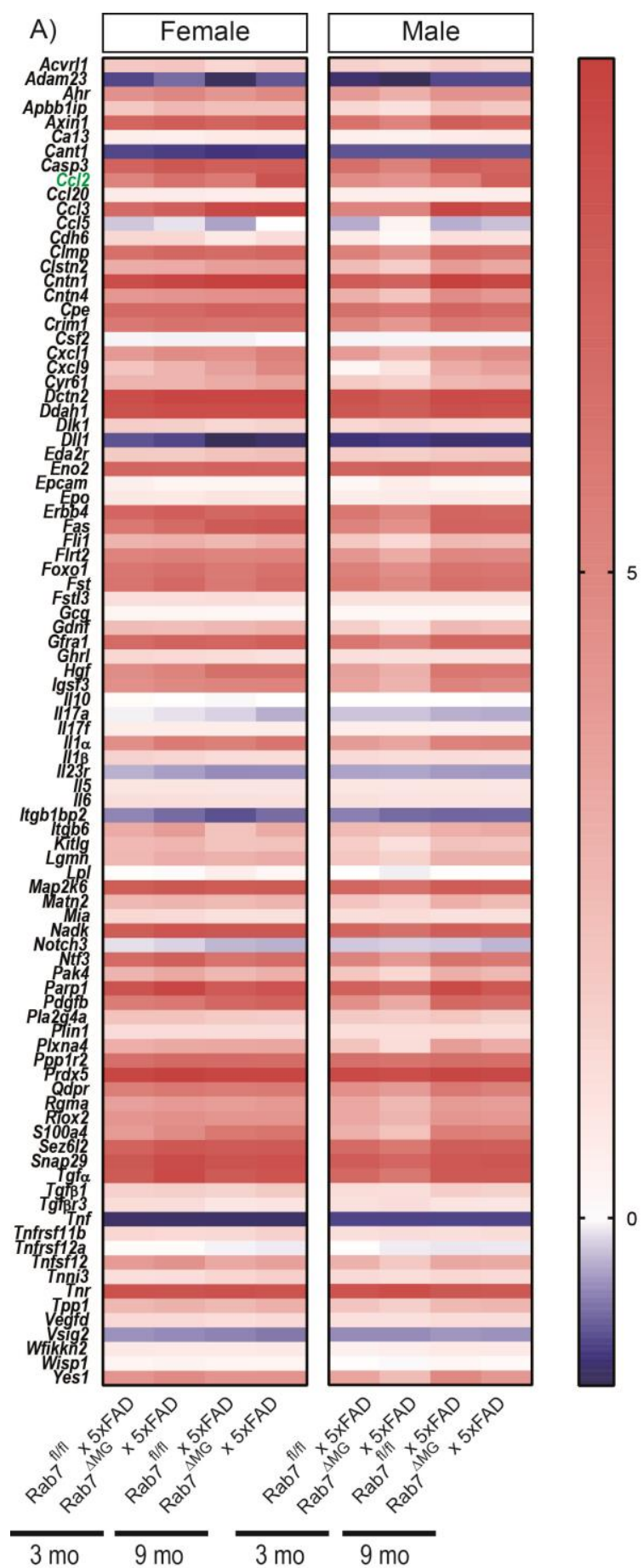

B)

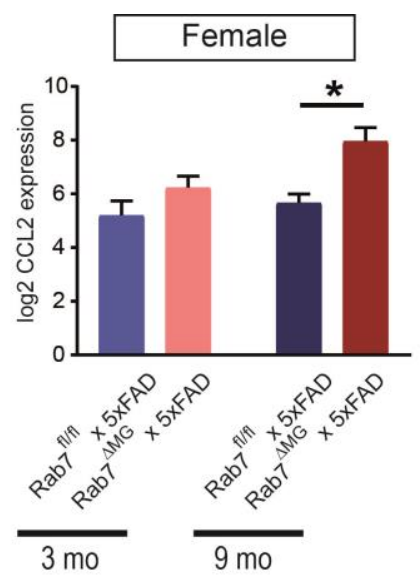

C)

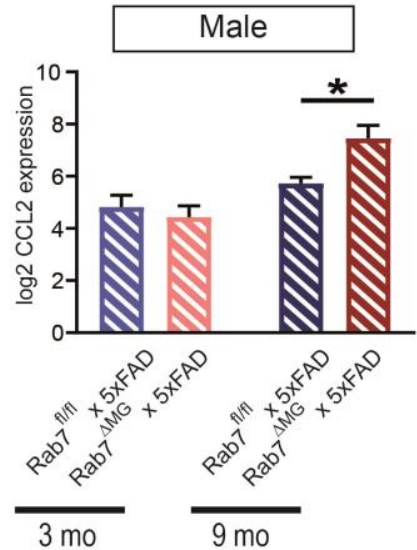

Figure 30: Protein profiling identified upregulation of CCL2 in aged Rab7 $7^{\triangle M G} \times 5 \times F A D$ mice.

A) Heatmap of all proteins analyzed by Olink® MOUSE EXPLORATORY. In the left panel the results of the female cohorts are shown, in the right panel the results of the male cohorts are presented. From left to right within the panel Rab7/t/1 $x$ 5XFAD and Rab7 ${ }^{\triangle M G} \times 5 \times F A D$ at 3 and 9 months, respectively. The scale is log 2 of the respective protein expression in a color code using red for upregulation and blue for downregulation. From the 3 month cohorts $n \geq 8$ animals and from the 9 month cohorts $\mathrm{n} \geq 6$ animals were analyzed. Differences between the genotypes were statistically analyzed by two-way ANOVA with Tukey's multiple comparisons test. Significant upregulation in Rab7 ${ }^{\triangle M G} \times 5 \times F A D$ animals was found for CCL2 $\left({ }^{*} p<0.05\right.$; green). B) Absolute values of CCL2 in females at 3 and 9 months of age are displayes. C) Graph shows CCL2 levels in males. Two-way ANOVA with Tukey's multiple comparisons test. Data are presented as mean \pm SEM. ${ }^{*} p<0.05$. 


\subsection{Rab7 $7^{\triangle M G}$ cannot be compensated by other brain cells in the 5xFAD mouse model}

Throughout $A D$ disease progression Rab7 $\triangle M G$ x $5 \times F A D$ female mice showed fewer and morphologically altered $A \beta$-plaques compared to Rab7 $7^{1 / f l} \times 5 \times F A D$ controls (Figure 17, Figure 18) while revealing no differences in soluble and insoluble $A \beta$-load (Figure 20-Figure 23). Since the Rab7 $\triangle M G$ results in impaired protein degradation through the endo-lysosomal pathway in microglia, there could be other ways for the CNS to remove $A \beta$-deposits, explaining the above mentioned effects on $A \beta$-plaque pathology. Therefore, we investigated the impact of other glial cells, namely astrocytes, on the removal of $A \beta$. Also, the possibility of $A \beta$ clearance by blood circulation was analyzed since endothelial $A \beta$-depositions are often found in blood vessels of $A D$ post mortem samples.

\subsubsection{Astrogliosis was not affected in Rab7 ${ }^{\Delta M G}$ x 5xFAD mice}

In neurodegenerative diseases such as $A D$ reactive astrogliosis is a prominent feature. Astrogliosis is defined as the response of astrocytes to injury by increased cell proliferation / numbers and morphological changes of the cells. Additionally, there is emerging evidence, that microglia and astrocytes communicate during diseases. Therefore we checked whether the Rab7 $\triangle \mathrm{MG} \times 5 \times \mathrm{xAD}$ had an impact on astrogliosis. To address this immunohistochemistry was done for the astrocytic marker GFAP (Glial Fibrillary Acidic Protein) on brain sections of 9 month old females (Figure 31 A-B) and males (Figure $\mathbf{3 1}$ E-F). In terms of absolute cell numbers of GFAP+ astrocytes normalized to the analyzed area, there was no detectable difference between Rab7 ${ }^{f / 1 / 1} \times 5 \times F A D$ and Rab7 $^{\triangle M G} \times 5 \times F A D$ females in the cortex (Figure 31 C) and in the hippocampus (Figure $31 \mathrm{D}$ ). Also male mice did not show any differences neither in the cortex (Figure $\mathbf{3 1} \mathbf{G}$ ) nor in the hippocampus (Figure $31 \mathrm{H}$ ) in GFAP+ astrocyte numbers. These findings indicate that Rab7 ${ }^{\triangle M G} \times 5 \times F A D$ has not impact on astrogliosis in progressed AD. 

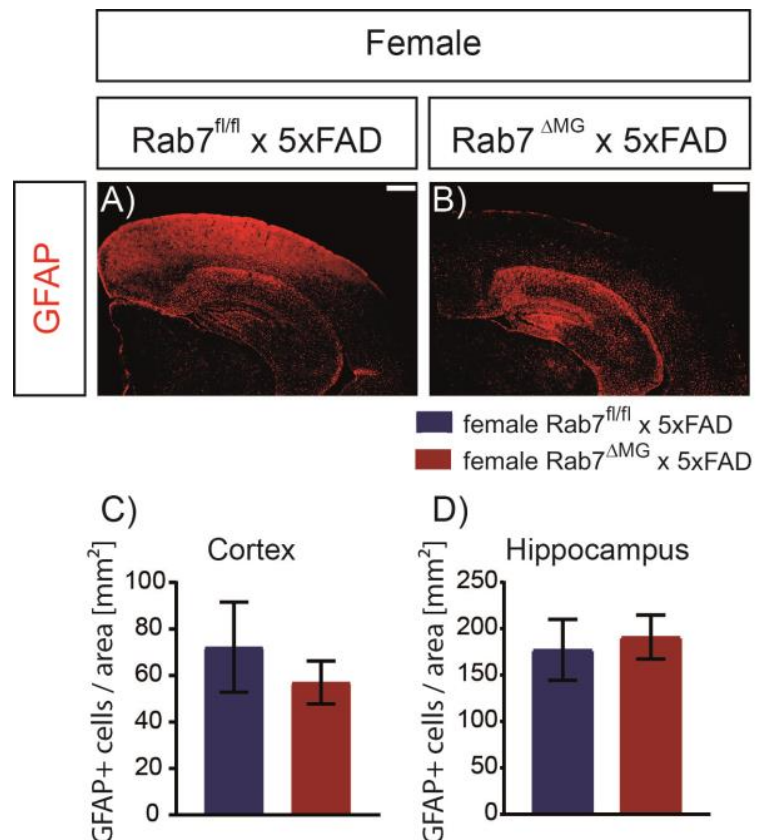

D)

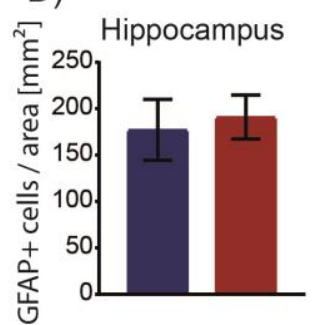

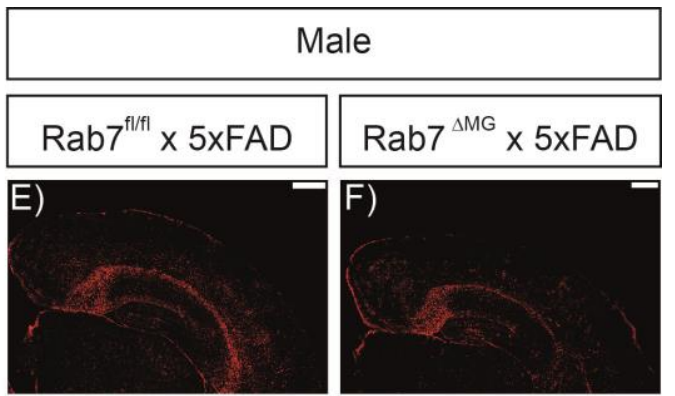

Wale $R a b 7^{\text {fl/fil }} \times 5 \times F A D$

$\mathbf{W}$ male Rab7 ${ }^{\mathrm{MMG}} \times 5 \times$ FAD

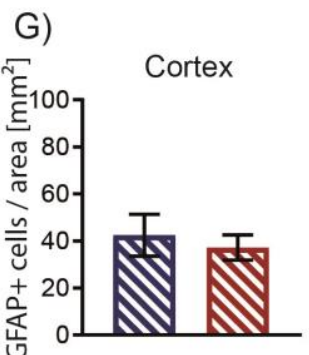

$\mathrm{H})$

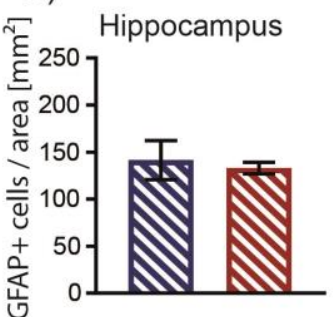

Figure 31: Astrogliosis was not changed in Rab7 ${ }^{\triangle M G}$ x 5xFAD mice.

A+B) Representative images show immunohistochemical stainings for astrocytic marker GFAP (red) on brain sections of 9 month old Rab7 $7^{\mathrm{fl} / \mathrm{fl}} \times 5 \times \mathrm{FAD}$ and Rab7 ${ }^{\triangle \mathrm{MG}} \times 5 \times \mathrm{xAD}$ females. Scale bar: $500 \mu \mathrm{m}$. C+D) Numbers of GFAP+ cells / area [mm²] were quantified in Rab7 $7^{\mathrm{fl} / \mathrm{fl}} \times 5 \times \mathrm{xAD}$ (blue) and Rab7 ${ }^{\triangle M G} \times 5 \times F A D$ (red) females in the cortex and the hippocampus, respectively. E+F) Immunohistochemistry for GFAP on brain sections of 9 month old Rab7 $7^{\text {fl/fl }} \times 5 \times F A D$ and Rab7 ${ }^{\triangle M G}$ x 5xFAD males are shown. Scale bar: $500 \mu \mathrm{m} . \mathrm{G}+\mathrm{H}$ ) Graphs show GFAP+ cells / area [mm²] quantified in the cortex and in the hippocampus for Rab7 $7^{\text {fl/fl }} \times 5 \times$ FAD (blue stripes) and Rab7 ${ }^{\triangle M G} \times 5 \times F A D$ (red stripes) males. For both sexes $n \geq 7$ animals with $N=2$ sections per animal were analyzed. Data are presented as mean \pm SEM. Analysis was done by Pascal Kalbhen.

\subsubsection{No compensation of $A \beta$-degradation by astrocytes was found in Rab7 ${ }^{\Delta M G}$ x 5xFAD females}

Even though we did not find astrocyte numbers to be changed in progressed $A D$ in the Rab7 ${ }^{\Delta M G} \mathrm{X}$ 5XFAD mouse line, we still wondered whether astrocytes could functionally compensate for impaired microglia in $A \beta$-degradation. This could be one mechanism to explain the smaller and fewer $A \beta$-plaques in female Rab7 ${ }^{\triangle M G} \times 5 \times F A D$ mice (Figure 17 - Figure 18). To address this, immunohistochemical co-staining for astrocytes (GFAP), lysosomes (LAMP1) and $A \beta_{42}$ was done and the degree of lysosomal engulfed $A \beta_{42}$ was measured (Figure $32 \mathbf{A}-\mathbf{H}$ ). We could not detect differences in the $A \beta_{42}$ fluorescence intensity engulfed in astrocytic lysosomes (Figure $32 \mathrm{I}$ ). Also measuring the relative area occupied by $A \beta_{42}$ within astrocytic lysosomes, did not change between $R a b 7^{f / / f l} \times 5 \times F A D$ and $R a b 7^{\triangle M G} \times 5 \times F A D$ females (Figure $32 \mathrm{~J}$ ). 


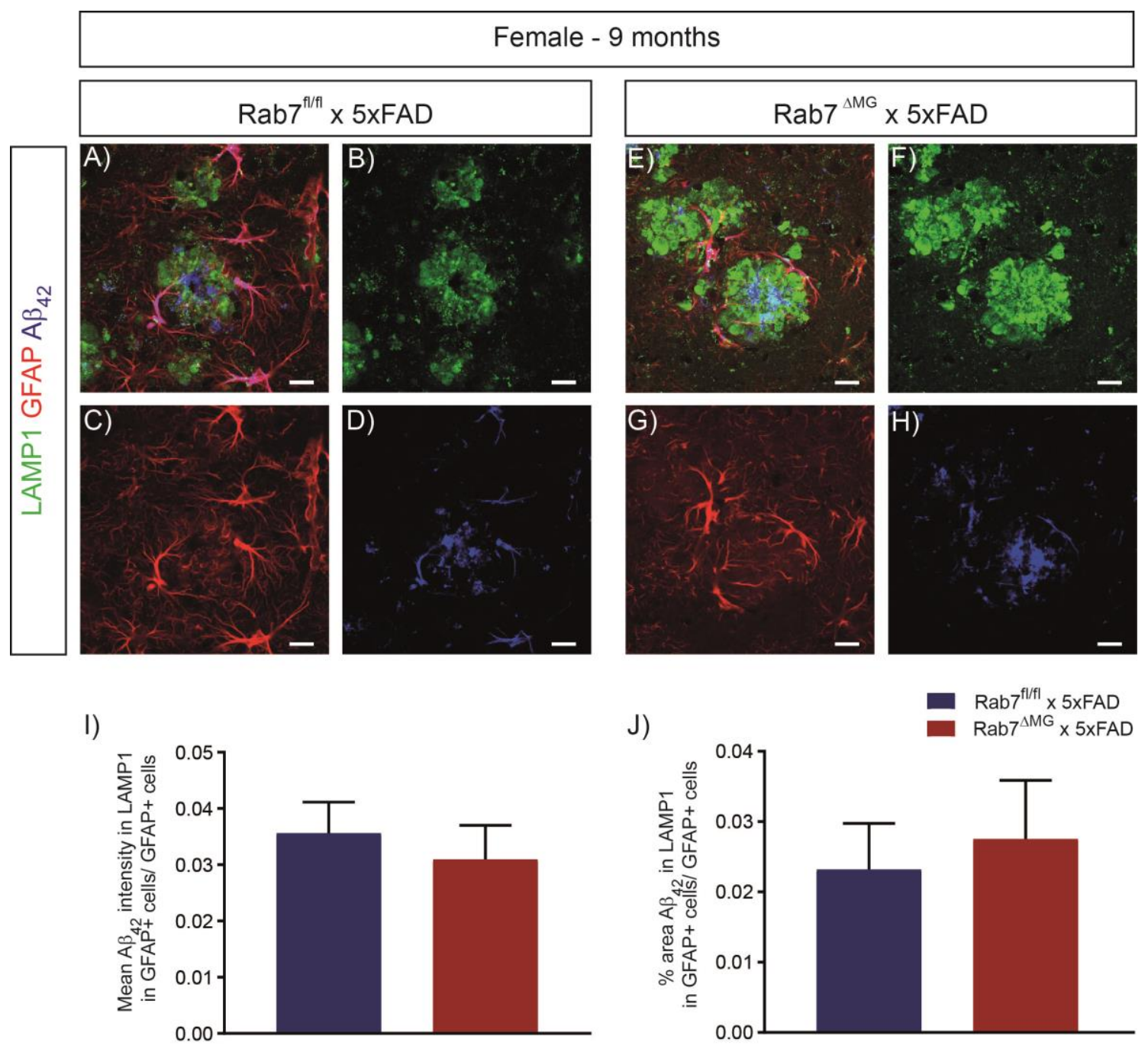

Figure 32: Astrocytes did not compensate for Rab7 ${ }^{\Delta M G}$ in clearing $A \beta$.

A-H) Brain sections of 9 month old females were stained by immunohistochemistry for LAMP1 (green), GFAP (red) and $A \beta_{42}$ (blue). Images were taken within cortex layer $\mathrm{V}-\mathrm{VI}$ above CA1 region with a 40x maginification using a confocal microscope. $\mathbf{A}+\mathbf{E})$ Merge images of $\mathbf{B}+\mathbf{F}$ ) LAMP1, $\mathbf{C}+\mathbf{G}$ ) GFAP and $\mathbf{D}+\mathbf{H}) A \beta_{42}$ co-stainings are presented. Scale bar: $10 \mu \mathrm{m}$. I) Fluorescence intensity of $A \beta_{42}$ within LAMP1+ vesicles inside GFAP+ astrocytes was quantified. J) As additional readout the relative area of $A \beta_{42}$ inside $L A M P 1+$ structures within a GFAP+ astrocyte was measured. Values of $R a b 7^{f / f \mid} \times 5 \times F A D$ controls are shown in dark blue and values of Rab7 ${ }^{\triangle M G} \times 5 \times F A D$ females are shown in red. Data are presented as mean \pm SEM. $n \geq 9$ animals; $N \geq 92$ cells analyzed.

\subsubsection{Plasma levels of $A \beta$ were not changed in $R a b 7^{\Delta M G} x$ 5xFAD mice}

Another possible pathway to help clearing $A \beta$-deposits from the brain is via the blood stream. This could be one possible mechanism to explain differences in A $\mathrm{Rab}^{\mathrm{ft/fl}} \times 5 \times \mathrm{xFD}$ and Rab7 ${ }^{\triangle M G} \times 5 \times F A D$ mice and between the sexes, respectively. To investigate 
this possibility plasma from 9 month old animals was analyzed for the presence of three $A \beta$ peptides by MSD chemiluminescent ELISA.

There were no differences found in the plasma levels of $A \beta_{38}, A \beta_{40}$ and $A \beta_{42}$ between $R a b 7^{f / 1 / f 1} x$ 5xFAD and Rab7 ${ }^{\triangle M G} \times 5 \times F A D$ animals of both sexes, respectively (Figure 33 A-C, E-G). Also A $\beta_{42}$ / $A \beta_{40}$ ratios were not altered between the conditions neither in females (Figure $33 \mathbf{D}$ ) nor in males (Figure $33 \mathbf{H}$ ). The absolute values of $A \beta_{38}, A \beta_{40}$ and $A \beta_{42}$ were similar in females and males as well. Consequently, alterations in the $A \beta$ clearance by blood cannot explain the differences in $A \beta$ plaque deposition observed in female Rab7 $7^{\mathrm{flfl}} \times 5 \times \mathrm{FAD}$ and Rab7 ${ }^{\triangle M G} \times 5 \times F A D$ mice.

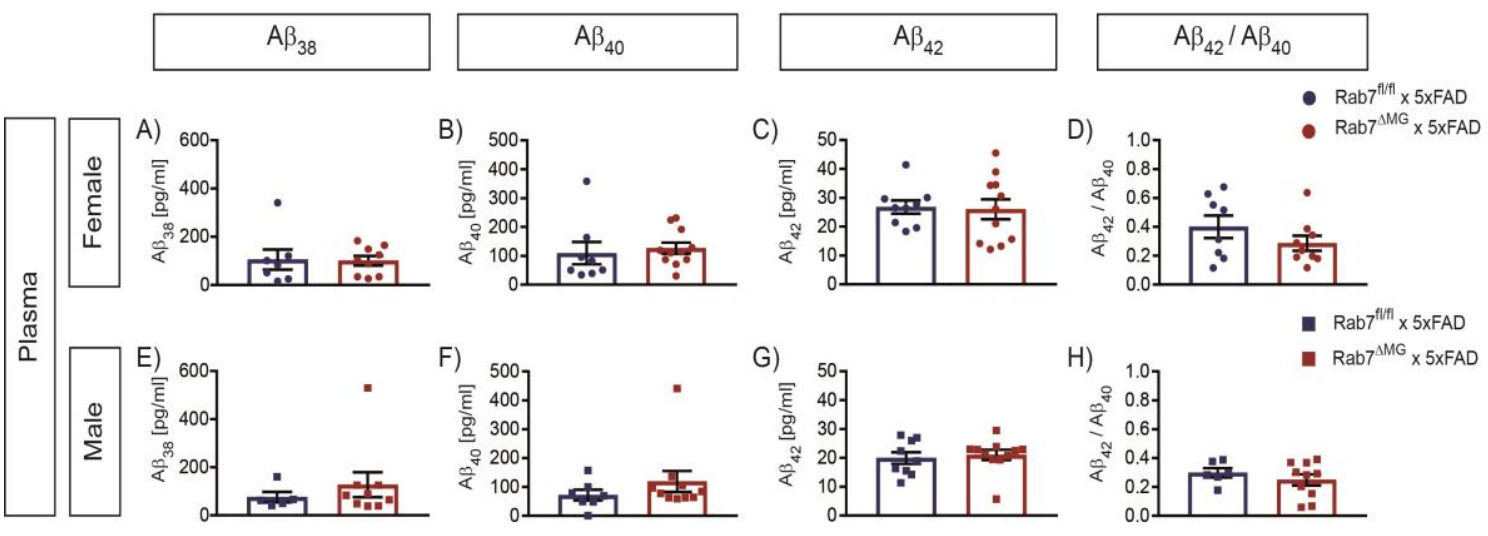

Figure 33: Clearance of $A \beta$ by the blood circulation is not altered in Rab7 ${ }^{\triangle M G} \times 5 \times F A D$ mice.

A-D) Analysis for $A \beta$-species in plasma of 9 month old females of the Rab7 $\triangle M G=5 x F A D$ line (red) wih their respective controls (blue) by MSD. E-H) A $\beta$-amounts detected in plasma from the 9 month male cohort. The absolute plasma concentrations of $\left.\mathbf{A}+\mathbf{E}) \quad A \beta_{38}, \mathbf{B}+\mathbf{F}\right) A \beta_{40}$ and $\left.\mathbf{C}+\mathbf{G}\right) A \beta_{42}[\mathrm{pg} / \mathrm{ml}$ plasma] were measured. $\mathbf{D}+\mathbf{H}) A \beta_{42} / A \beta_{40}$ ratios were calculated for females and for males. Graphs are presented as mean \pm SEM with the individal samples added to the blot as circles (females) or squares (males). $\mathrm{n} \geq 8$ animals. Measurements in duplicates. 


\section{Discussion}

To elucidate whether microglial endo-lysosomal degradation is beneficial or detrimental in $A D$, we have used a microglia-specific conditional knockout of small GTPase Rab7 (Rab7 ${ }^{\triangle M G}$ ), which is crucial for endo-lysosomal maturation, in 5xFAD mice (Rab7 $\triangle M M G \times 5 \times F A D)$ and investigated these mice at 3 and 9 months, respectively.

Surprisingly, we found fewer and smaller A $\beta$-plaques in female 5xFAD mice with Rab7 KO microglia while no effect on overall soluble and insoluble forms of $A \beta$-peptides and only moderate amelioration on dendritic spine loss were found in 9 months old Rab7 ${ }^{\triangle M G} \times 5 \times F A D$ female mice. Interestingly, we found upregulation of chemokine CCL2 in Rab7 $\triangle M G$ x $5 x F A D$ mice of both sexes.

\subsection{Evaluation of the microglial Rab7 KO in 5xFAD mice}

Given that microglial lysosomal degradation is considered the main pathway for extracellular $A \beta$-clearance, it was startling to only detect moderate effects on $A \beta$-pathology while no improvement on neuronal loss was found in Rab7 $\triangle M G G \times 5 \times F A D$ mice. Hence, to assure the functionality of our tamoxifen-induced conditional microglial Rab7 KO, we quantified accumulation of $A \beta_{42}$ in microglial endo-lysosomes and found significant enrichment in Rab7 ${ }^{\triangle M G} \times 5 \times F A D$ mice, which substantiates the functional impairment in our model (Figure 16). Alongside, we detected enlarged lysosome volumes by immunohistochemistry for lysosomal markers LAMP1 and CD68 due to the lack of immunohistochemistry compatible antibodies against Rab7 (Figure 14, Figure 15). Our findings that lysosome volumes were enlarged in Rab7 $\triangle M G$ x $5 \times F A D$ microglia are in accordance with data of Safaiyan et al. (2016) who investigated Rab7 KO on myelin turnover using the same Cre and Rab7 fl/fl mouse lines. Notably, a recent report suggested that the $\mathrm{CX}_{3} \mathrm{CR} 1$ -

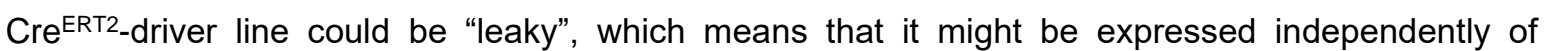
tamoxifen application (Fonseca et al., 2017). However, the genetic background of the $\mathrm{CX}_{3} \mathrm{CR} 1$ Cre ${ }^{\text {ERT2}}$-driver used by Fonseca and colleagues (2017) was different from the $\mathrm{CX}_{3}$ CR1-Cre ${ }^{\text {ERT2 }}$ driver used by Safaiyan et al. (2016) and in this study. Additionally, we found significant enlargement of endo-lysosomes with $A \beta_{42}$ accumulations in microglia of Rab7 $\triangle M G$ $\times 5 \times F A D$ females compared to Rab7 $7^{1 / f \mid l} \times 5 \times F A D$ controls at 9 months of age (Figure 14, Figure 15). Thus, we concluded that Rab7 $\triangle M G$ x 5xFAD mice displayed Rab7 dysfunction, even if there might be moderate and comparably low Cre-recombination in Rab7 fl/fl $\times 5 \times F A D$ controls. To exclude the possibility of $\mathrm{CX}_{3} \mathrm{CR} 1-\mathrm{Cr} \mathrm{ERT2}^{\mathrm{ER}}$ leakiness in our model, Rab7 transcripts and protein levels of purified microglia need to be quantified by qPCR and Western blot analysis in future experiments. 


\subsection{Compensatory mechanisms for Rab7 $\mathrm{KO}$ in microglia}

To our surprise, Rab7 ${ }^{\triangle M G} \times 5 \times F A D$ mice showed smaller and fewer $A \beta$-plaques in females at 9 months of age (Figure 18) while we detected only improvement in loss of mushroom spines with no overall reduction of neuron loss (Figure 24 - Figure 26). Therefore we addressed whether other cellular, enzymatic or transport mechanisms could compensate for microglial $A \beta$-clearance in $\mathrm{Rab}^{\triangle \mathrm{MG}} \times$ x $\mathrm{xFAD}$ mice.

\section{a) Peripheral monocytes and perivascular macrophages}

Aside from microglia, peripheral derived immune cells, including monocytes, neutrophils and T-cells have been detected in human post mortem samples of $A D$ patients and in rodent models. These cells are thought to aid directly or indirectly in clearing $A \beta$ (Baik et al., 2014; Itagaki et al., 1988; Vogel et al., 2015; Zenaro et al., 2015). Hence, Rab7 $\triangle M G$ could initiate the infiltration of other myeloid cells for functional compensation. In favor of this idea are the findings of elevated levels of chemokine CCL2 (CC-chemokine ligand 2) or MCP1 (monocyte chemotactic protein 1) in hippocampal lysates of 9 months old Rab7 $\triangle \mathrm{MG} \times 5 \times \mathrm{FAD}$ mice compared to their respective controls (Figure 30). CCL2 is able to attract CCR2-expressing peripheral myeloid cells e.g. from the blood stream which is facilitated throughout disease progression by the gradual breakdown of the blood brain barrier (Malm et al., 2005; Roberts et al., 2012; Simard et al., 2006; Stalder et al., 2005; Varvel et al., 2016). CCR2+ cells were identified in various AD mouse models with progressed pathology in close proximity to $A \beta$-plaques contributing to $A \beta$-clearance (El Khoury et al., 2007; Naert and Rivest, 2011; Saederup et al., 2010). However, fate-mapping studies showed that neither microglia nor other long-lived Iba1+, CX3CR1+ CNS macrophages like perivascular and meningeal macrophages, express CCR2 (Goldmann et al., 2016; Mizutani et al., 2012; Yona et al., 2013). Thus, the enriched levels of CCL2 very likely contribute to the infiltration of CCR2+ peripheral myeloid cells. This idea is further supported by a current study that discovered small vascular channels within the skulls of mice and humans allowing for skull bone marrow-derived myeloid cells, including CCR2-expressing monocytes and neutrophils to rapidly enter the inflamed brain (Herisson et al., 2018). Hence, follow-up experiments for cell infiltration, e.g. by immunohistochemistry for CCR2 and lba1 should be done. To investigate compensation of $A \beta$-clearance e.g. by immunohistochemistry for CCR2, LAMP1 and $A \beta_{42}$, should be conducted in the future.

Besides peripheral monocytes, perivascular macrophages could potentially compensate for Rab7 $\mathrm{KO}$ microglia by various mechanisms and therefore could explain the moderate effects on neuronal loss and $A \beta$-pathology in our model. Perivascular macrophages, which are located along blood vessels, are well described as gatekeepers at the blood brain barrier where they monitor and 
promote communication with the periphery and the CNS. By expression of CCL2 and GM-CSF, molecules which were shown to aid in blood monocytes migration across the blood brain barrier, these cells could actively promote infiltration of CCR2-expressing peripheral monocytes (Bechmann et al., 2001; Varvel et al., 2016; Vogel et al., 2015). Even though these cells have high phagocytic activity and are also capable of degrading $A \beta$, they are long-lived and express $C X_{3} C R 1$ (Goldmann et al., 2016; Hawkes and McLaurin, 2009; Mato et al., 1985; Mato et al., 1996; Thanopoulou et al., 2010). Hence, it is likely that Rab7 $\triangle M G$ x $5 \times F A D$ mice are also Rab7 depleted in perivascular macrophages. Due to overlapping markers such as Iba1 and CD68, these cells could potentially contribute to the pool of Iba1+ cells (Figure $27 \mathrm{I}, \mathbf{J}, \mathbf{U}, \mathbf{V}$, Figure $28 \mathrm{I}, \mathbf{J}, \mathbf{U}, \mathbf{V}$ ) and Aß-plaque associated Iba1 + cells (Figure 27 K, L, W, X, Figure 28 K, L, W, X) which we found to be unvaried in Rab7 $\triangle \mathrm{MG}$ x 5xFAD and Rab7 $7^{\text {fl/fl }}$ x 5xFAD mice (Fabriek et al., 2005; Goldmann et al., 2016; Kim et al., 2006; Zeisel et al., 2015). Consequently, it will be of great importance to distinguish microglia from perivascular macrophages and from infiltrating peripheral monocytes. Thus, further experiments such as immunohistochemistry for microglia-specific marker TMEM119, perivascular maker CD163 and monocyte-marker CCR2 will need to be performed in the future.

\section{b) A $\beta$-degrading enzymes}

Besides degradation of $A \beta$ by intracellular mechanisms, microglia can also mediate clearance of soluble $A \beta$ by secreting various enzymes with $A \beta$-degrading capacity into the extracellular space. These enzymes include amongst others neprilysin (NEP), endothelin converting enzymes 1 and 2 (ECE-1 and ECE-2), insulin degrading enzyme (IDE or isulysin), matrix metallopeptidases 2 and 9 (MMP2, MMP9) and serine protease tissue plasminogen activator (tPA) (Hernandez-Guillamon et al., 2010; Iwata et al., 2001; Melchor et al., 2003; Song and Hersh, 2005; Yan et al., 2006). Moreover, lysosomal protease Cathepsin B (Cat B) is secreted via exocytosis into the extracellular space where it is associated with senile plaques and promotes neuroprotection by proteolytic cleaving of $A \beta_{42}$ in APP-overexpressing mice (Cataldo and Nixon, 1990; Linebaugh et al., 1999; Mueller-Steiner et al., 2006; Sun et al., 2008). However, activity of those enzymes can be modulated by intrinsic enzyme inhibitors. This includes, amongst others, late onset AD risk gene cystatin C which acts as inhibitor of Cathepsin B. Additionally, serine protease inhibitors a1antichinotrypsin and $\alpha 1$-antichymotrypsin favor $A \beta$-fibril formation while inhibiting $A \beta$-plaque degradation (Abraham et al., 2000; Abraham et al., 1989; Bertram et al., 2007; Fraser et al., 1993; Sun et al., 2008). To exclude the possibility that $A \beta$-plaque reduction (Figure 17, Figure 18) was mediated by extracellular degradation by such enzymes, follow-up experiments are required such as quantification of NEP, IDE and MMPs by Western blotting.

\section{c) A $\mathrm{\beta}$-clearance across the blood brain barrier}

Another clearance mechanism of extracellular $A \beta$-peptides, which could compensate for Rab7 $\triangle \mathrm{MG}$ in $5 x F A D$ mice, is through efflux of soluble $A \beta$-peptides across the blood brain barrier into the blood 
stream (Keaney et al., 2015; Storck et al., 2016). Therefore, macromolecules such as apolipoproteins ApoE, ApoJ (also known as clusterin, CLU), tPA and pan-protease inhibitor $\alpha 2$-macroglobulin (A2D) can bind monomeric and oligomeric $A \beta$-peptides and facilitate efflux across the blood brain barrier (Beeg et al., 2016; Biere et al., 1996; Bobkova et al., 2014; Hoshino et al., 2013; Iwata et al., 2013; Iwata et al., 2001; Melchor et al., 2003; Narayan et al., 2011; Yepes et al., 2003). The guided transport is mediated through lipoprotein receptor-related protein 1 and 2 (LRP1, LRP2) which are abundantly expressed within small brain vessels (Rebeck et al., 1995). For instance, ApoE2 and ApoE3 bind $A \beta_{40}$ and shuttle via LRP1, whereas ApoJ-attaches to $A \beta_{42}$ and uses LRP2 to cross the blood brain barrier (Bell et al., 2007; Shibata et al., 2000). In our study we did not detect differences in the levels of soluble $A \beta_{38}, A \beta_{40}$ or $A \beta_{42}$ in collected plasma of $R a b 7^{f / / 1 / 1} x$ 5xFAD and Rab7 $\triangle M G$ x 5xFAD animals at 9 months of age (Figure 33). Therefore, we suggested that microglial Rab7 KO probably did not impact the efflux of $A \beta$-peptides across the blood brain barrier. However, steady state levels of plasma $A \beta$ may not accurately reflect clearance kinetics, as $A \beta$ is efficiently cleared from the blood stream by the renal system.

\section{d) Upregulation of other degradation pathways in microglia}

Interestingly, ApoJ, ApoE and A2D also belong to a group of extracellular chaperone proteins. Similarly to intracellular chaperons such as heat shock protein 70 (Hsp70), extrinsic chaperones bind to proteins and thereby form a stable complex. This facilitates trafficking, as described for the efflux across the blood brain barrier, but also helps cellular internalization for intracellular A $\beta$-degradation (Boland et al., 2018; Cascella et al., 2013; Yeh et al., 2016). In microglia, these pathways are mainly autophagy and endo-lysosomal degradation, which were both shown to be important in AD. Genetic and pharmacological disruption of autophagosome formation in myeloid cells in several $A D$ transgenic mouse models reduced $A \beta$-burden while cognitive functions were improved (Caccamo et al., 2010; Cho et al., 2014; Kim et al., 2017; Spilman et al., 2010). These modulations were upstream of Rab7, which is only detectable at stages of late autophagosomes and late endosomes, and are essential for the fusion with the lysosome as illustrated in Figure $\mathbf{1 0}$ (Cantalupo et al., 2001; Gutierrez et al., 2004; Kuchitsu et al., 2018). Since we validated vesicle enlargement in our Rab7 $\triangle M G$ x $5 \times$ FAD model by immunohistochemical staining for LAMP1, which is also expressed in the late stages of autophagy, we cannot draw conclusions about upstream early autophagosome formation (Figure 14, Figure 15). Since KO of Trem2, which is upstream of Rab7 and is responsible for the formation of endo-lysosomal active DAM, restored autophagy by suppressing mTor signaling in microglia of aged 5xFAD mice, it could be possible that early autophagosome formation was also enhanced in microglial Rab7 KO mice (Ulland et al., 2017). To address this question, further analysis would be needed.

Similar to the disruption of autophagosome formation, ablating phagocytosis through $\mathrm{KO}$ of complement factors improved cognitive performance and reduced A $\beta$-pathology (Fonseca et al., 2004; Hong et al., 2016; Paolicelli et al., 2011; Shi et al., 2017). Concomitantly, endo-lysosomal 
degradation of Rab7 is crucial for the final fusion step with the lysosome. In the present work, we did not further investigate for alterations within the upstream endocytic pathway including early endosome formation and phagocytosis in Rab7 ${ }^{\triangle M G} \times 5 \times F A D$ mice.

\section{e) Astrocytes}

Other than microgliosis, astrogliosis is also a prominent hallmark of $A D$ and reactive astrocytes are found in most $A \beta$-rich brain regions of $A D$ post mortem samples and in transgenic $A D$ mice (Gomez-Arboledas et al., 2018; Liddelow et al., 2017; Reichenbach et al., 2018). Similarly to microglia, reactive astrocytes can contribute to neurotoxicity while also supporting $A \beta$-clearance through endo-lysosomal degradation (Boisvert et al., 2018; Funato et al., 1998; Jones et al., 2013; Kamphuis et al., 2015; Kraft et al., 2012; Nagele et al., 2003; Söllvander et al., 2016; Xiao et al., 2014). Even though microglia and astrocytes are known to tightly interact with each other, we did not find changes in astrogliosis indicated by unvarying numbers of GFAP+ cells within the cortex and the hippocampus in 9 months old Rab7 $7^{\mathrm{fl} / \mathrm{fl}} \times 5 \times \mathrm{FAD}$ and Rab7 ${ }^{\triangle M G} \times 5 \times F A D$ females and males, respectively (Figure 31). Neither did we find differences in enrichment of $A \beta_{42}$ in astrocytic lysosomes (Figure 32). Since astrocytes were also demonstrated to have slow lysosomal degradation, which results in accumulation of $A \beta_{42}$ in enlarged lysosomes in $A D$, our findings of unaffected $A \beta_{42}$ in astrocytic lysosomes indicated that Rab7 $\triangle M G$ was not likely to be compensated by astrocytes (Söllvander et al., 2016). It needs to be mentioned that this quantification does not allow for conclusions to be drawn about the dynamics and speed of $A \beta$-degradation. Since the speed of astrocytic endo-lysosomes could be increased in vitro, it still could be possible that astrocytes compensate for Rab7 ${ }^{\triangle M G}$ by faster endocytic vesicle trafficking (Lööv et al., 2015). Thus, to elucidate astrocytic degradation speed, further experiments such as live cell imaging of mixed astro-microglial cultures would need to be conducted.

\subsection{Effects of microglial Rab7 KO on amyloid- pathology and neurotoxicity in 5xFAD mice}

In our model of microglial Rab7 KO in 5xFAD mice, we found improvement on mushroom spine loss and fewer and smaller $A \beta$-plaques with overall unaffected insoluble and soluble $A \beta$-burden. Aside from those findings, our data is in contrast to studies which investigated acute and chronic total microglia depletion by pharmacological inhibition and which reported of strong neuroprotection in mice whilst no effect on A -burden was found (Dagher et al., 2015; Olmos-Alonso et al., 2016; Spangenberg et al., 2016). These studies suggested that $A \beta$-deposition was independent of the presence of microglia. For example, genetic ablation of microglia did not change $A \beta$-load in 3 months old APP/PS1 males (Grathwohl et al., 2009), and pharmacological ablation of microglia by CSF1 inhibitor treatment did not result in altered $A \beta$-burden of neither soluble nor insoluble 
Aß-species in 3xTg, APP/PS1 and 5xFAD mice (Dagher et al., 2015; Olmos-Alonso et al., 2016; Spangenberg et al., 2016).

\section{Time-dependency on A $\beta$-pathology}

One fundamental difference of our study to those studies investigating total microglia depletion was timing. In our model, microglial Rab7 KO was already induced with 3 weeks, even before transcriptional changes of innate immune genes in microglia and intraneuronal $A \beta$ were found in 5xFAD mice (Boza-Serrano et al., 2018; Oakley et al., 2006). This is in contrast to the studies using pharmacological inhibition of myeloid cell receptor CSF1 which did not change A $\beta$-burden, and started treatment only at ages when the investigated mouse lines already displayed abundant A $\beta$-pathology (Dagher et al., 2015; Olmos-Alonso et al., 2016; Spangenberg et al., 2016). As longterm in vivo imaging data of APP/PS1 mice revealed that new A $\beta$-plaques were not formed after 9 months of age, this emphasizes the importance of time in modulaing $A \beta$-pathology (Hefendehl et al., 2011).

Therefore, we assumed that constitutive genetic ablation of Trem2, which is upstream of Rab7 and essential for the second step in formation of highly phago-lysosomal active DAM, would result in a similar phenotype than our Rab7 ${ }^{\Delta M G}$ x 5xFAD model (Jay et al., 2017; Jay et al., 2015; Keren-Shaul et al., 2017; Krasemann et al., 2017; Wang et al., 2015; Wang et al., 2016). In accordance to Trem2 KO in 5xFAD, our microglial Rab7 KO in young (3 months) 5xFAD mice also did not display statistical differences in $A \beta$-plaque numbers or soluble and insoluble amounts of $A \beta_{40}$ and $A \beta_{42}$ (Figure 17, Figure 20) (Wang et al., 2016). However, aged Trem2 KOs (8-9 months) revealed increased numbers of $A \beta$-plaques and enriched $A \beta_{40}$ and $A \beta_{42}$ in hippocampal lysates, whereas we found fewer and smaller $A \beta$-plaques and no differences in the levels of soluble and insoluble $A \beta_{40}$ and $A \beta_{42}$ in 9 months old Rab7 ${ }^{\triangle M G}$ x 5xFAD females (Figure 17, Figure 21) (Wang et al., 2015b; Wang et al., 2016). Similarly, Trem2 KO mice revealed elevated inflammatory response and increased neuronal loss, whereas we did not observe either of these. Thus, our findings support the idea that initial $A \beta$-plaque formation is independent of microglia function, but throughout disease progression functional microglia are important to modulate $A \beta$-plaque pathology.

\section{Dendritic spine loss}

In consistency with pharmacological microglia depletion in 5xFAD mice, we also observed significantly more mushroom spines in 9 months old Rab7 $7^{\triangle M G} \times 5 \times F A D$ females while the overall number of dendritic spines was not changed (Figure 25) (Spangenberg et al., 2016). Methodologically, we used the same samples size and parameters to classify dendritic spines as Spangenberg and colleagues (2016). Mushroom spines, which are essential for memory formation, were found to be the most affected spine type in the presence of $A \beta$ in transgenic mice, explaining the AD-associated cognitive decline and memory loss (Luebke et al., 2010; Sun et al., 2014; Tackenberg and Brandt, 2009). Even though recent studies revealed high turnover rates of dendritic 
spines in physiological context using high resolution microscopy, mushroom spines were identified to be the most persistence spine type (Grutzendler et al., 2002; Pfeiffer et al., 2018). These dynamics of other spine types, in combination with the observation of targeted mushroom spine loss in $A D$, substantiates our findings of selective mushroom spine phenotype (Figure 25). Studies in macrophages have identified a NF-KB coupled feedback mechanism from the lysosome to sustain phagocytosis of bacteria (Wong et al., 2017). Thus, we cannot exclude the possibility that such a mechanism also exists for microglial phagocytosis of $A \beta$-peptides and Rab7 KO results in reduced phagocytosis through a negative feedback mechanism. It is therefore possible that microglial Rab7 KO and subsequent accumulation of undigested $A \beta$ in microglia decrease their phagocytic activity also towards spines. This may explain the protective effect of microglial Rab7 KO. Since loss of dendritic spines and of synapses correlate with impaired synaptic plasticity, resulting in memory loss and cognitive deficits, electrophysiological features such as long-term potentiation should be analyzed in the future. Additionally, behavior analyses such as novel object recognition, conditional fear conditioning or Morris water maze would have been advisable to gain further insight into potentially neuroprotective effects in Rab7 ${ }^{\triangle M G} \times 5 \times F A D$ mice.

\section{Synaptic loss}

In our model we did not determine changes in the degree of synaptic loss between 9 months old $R a b 7^{f / 1 / 11} \times 5 \times F A D$ and Rab7 ${ }^{\triangle M G} \times 5 \times F A D$ mice, measured by protein levels of Synaptophysin (Figure 26). This is in contrast to studies which identified significant improvement of synaptic loss upon disruption of microglial phagocytosis e.g. by modulating factors of the complement system However, phagocytosis is upstream of Rab7 which could explain why microglial Rab7 KO was not sufficient to prevent from synaptic loss in our model.

\section{Neuronal loss}

Neurotoxicity is known to correlate with chronic inflammation and soluble A $\beta$-peptides (Lambert et al., 1998; Parajuli et al., 2013; Walsh et al., 2002; Yang et al., 2017; Zhao et al., 2018). Alongside increased amount of soluble $A \beta_{42}$ and elevated neuroinflammation, Trem2 $\mathrm{KO}$ in 5xFAD showed significantly more neuronal loss in cortical layer $\mathrm{V}$ in 8.5 months old mice compared to 5xFAD control, whereas we did not find alterations in soluble and insoluble $A \beta$-peptides, pro-inflammatory cytokines and neuronal loss (Figure 24) (Wang et al., 2015b). Several studies explicitly analyzed neuronal loss within cortical layer $\mathrm{V}$ of $5 x F A D$ mice, since they did not detect significant differences in overall cortical loss of neurons (Jawhar et al., 2012; Wang et al., 2015b). As we analyzed neuronal loss in all cortical layers and did not find significant amelioration in subicular neuronal loss due to one outlier in the female Rab7 ${ }^{\triangle M G} \times 5 \times F A D$ cohort, we cannot exclude the chance of reduced neuronal loss upon Rab7 KO in microglia of females (Figure 24). Hence, follow-up analysis with increased numbers of biological samples and analysis restricted to cortical layer $\mathrm{V}$ will need to be done. 


\section{Inflammation}

As mentioned before, neurotoxicity can either be mediated by soluble $A \beta$-species, which we did not find to be changed in Rab7 ${ }^{\triangle M G} \times 5 \times F A D$. Pro-inflammatory molecules can contribute to neurotoxicity including IL-1 $\beta$, IL-6, IL-10 and TNF- $\alpha$ which are highly abundant markers of Trem2-dependent DAM (Jay et al., 2017; Martin et al., 2017; Wang et al., 2015b; Wang et al., 2016). We did not find differences in the inflammatory profile between Rab7 $7^{\mathrm{fl/fl}} \times 5 \times \mathrm{FAD}$ and Rab7 ${ }^{\triangle \mathrm{MG}} \times 5 \times \mathrm{FAD}$ (Figure 30). In contrast, transcriptional analyses of Trem2 KO in 5xFAD and APP/PS1 mice showed diminished levels of pro-inflammatory cytokines including IL-1 $\beta$, IL-12 and TNF- $\alpha$ (Jay et al., 2015; Wang et al., $2015 b)$. For example, IL-1 $\beta$ is a product of the NLRP3 inflammasome, which can be activated by Trem2 and also by lysosomal rupture, which we assumed to be prevented in our Rab7 KO model due to inhibited endo-lysosomal fusion (Amaral et al., 2018; Halle et al., 2008; Heneka et al., 2012; Hornung et al., 2008; Jay et al., 2017; Martin et al., 2017).

Also $\mathrm{KO}$ of progranulin (PNG), which is a key regulator of inflammatory response in myeloid cells and which is associated with other forms of dementia, namely frontotemporal dementia and familial frontotemporal lobar degeneration, resulted in enlarged Rab7+ and LAMP1+ vesicles and upregulation of complement factors in microglia (Baker et al., 2006; Cruts et al., 2006; Lui et al., 2016; Rascovsky et al., 2011). This functional impairment coupled with enhanced secretion of proinflammatory cytokines IL-1 $1 \beta$, IL- 6 and TNF- $\alpha$ and reduced IL-10 expression after toxin or LSP stimulation in vitro (Lui et al., 2016; Martens et al., 2012; Yin et al., 2010). Taking these findings together, we suggest that microglial Rab7 KO reduces or even prevents lysosomal rupture and thereby diminishes the activation of NLRP3. Furthermore, this could potentially reduce pyroptotic microglial cell death, which in turn could contribute to further A $\beta$-plaque seeding (Venegas et al., 2017). To further elucidate this, follow-up experiments are needed to investigate the degree of inflammasome activation.

\section{Morphology}

Alongside inflammatory response, activated microglia undergo morphological changes towards amoeboid shape in AD (Davies et al., 2017; Navarro et al., 2018; Plescher et al., 2018). In vivo mouse studies observed that PAM actively degrade $A \beta$ and thereby grow in size while the attacked $A \beta$-plaque is shrinking (Bolmont et al., 2008). Thus, altered cell morphology is frequently used as a readout for microglia activation. In our model, we could not detect differences in the microglia morphology in neither PAM nor non-PAM between Rab7 $7^{\mathrm{fl} / \mathrm{fl}} \times 5 \times \mathrm{xFD}$ and Rab7 ${ }^{\triangle \mathrm{MG}} \times 5 \times \mathrm{xF}$ females at 9 months (Figure 29). This correlates to the findings of unaffected inflammatory response, concluding that microglial Rab7 KO were not impaired in their activity. Taking these findings together, microglia activity monitored by morphology and secretome analysis was not changed in microglia with Rab7 KO and therefore cannot explain the diminished $A \beta$-plaque numbers and sizes observed in 9 months old Rab7 $\triangle M G$ x 5xFAD females. 
Notably, Safaiyan and co-workers (2016) reported about significant shortening and swelling of microglia processes in the cortex of Rab7 ${ }^{\Delta \mathrm{MG}}$ mice compared to control animals at 10 weeks of age, concluding that Rab7 KO microglia have a higher basal activity compared to WT microglia.

\section{PAM recruitment}

Although we did not find differences in the load of soluble neurotoxic $A \beta_{42}$-peptides, nor in the inflammatory response, we detected reduced mushroom spine loss in 9 months old Rab7 $\triangle \mathrm{MG} x$ 5xFAD females (Figure 21, Figure 25, Figure 30). Dendritic loss is highly abundant in close proximity to Aß-deposits (Crowe and Ellis-Davies, 2014; Masliah et al., 1996; Spires et al., 2005; Tsai et al., 2004; Zhao et al., 2017). Whereas high aggregates of $A \beta$ are considered less toxic, $A \beta$-oligomers are considered to be of great neurotoxic potential (Lambert et al., 1998; Yang et al., 2017; Zempel et al., 2013). This neurotoxicity was shown to be reduced by PAM which can build functional barriers around $A \beta$-plaques to shield neurons from $A \beta$-deposits resulting in densely packed $A \beta$-plaques (Condello et al., 2015). Hence, loosely attached $A \beta$-fibrils around the dense core, which build up big halos, are considered more toxic than dense senile plaques. In accordance, Wang and colleagues $(2015 b ; 2016)$ identified bigger halos alongside increased neuronal loss and dystrophic neurites in their Trem2 KO. In contrast, we identified smaller $A \beta$-plaques with smaller halos, rescued loss of mushroom spines and unaffected neuronal loss as discussed before (Figure 18, Figure 24). Our findings are in line with the model of $A \beta$-plaque growth proposed by Baik and colleagues (2016): the authors identified accumulation of fibrillary $A \beta_{42}$ in lysosomes due to acidification which eventually contributed to lysosomal membrane rupture. This resulted in microglial apoptosis and consequently, release of undegraded $A \beta$ into the extracellular space (Yang et al., 1998). There the material was attached to pre-existing A $\beta$-plaques and contributed to $A \beta$-plaque growth. Since in the present work we ablated the fusion of the late endosome with the lysosome, this cascade should be abolished as well, which could explain the $A \beta$-plaque size reduction and the diminished number of $A \beta$-plaques due to fewer seeds in 9 months old Rab7 $\triangle M G$ x 5xFAD females.

Notably, other groups identified $A \beta$-plaque growth due to acute microglia depletion or to Trem2 KO in APP/PS1 (Jay et al., 2017; Jay et al., 2015; Zhao et al., 2017). These varying observations could be due to methodological differences to our study and the studies in Trem2 depleted 5xFAD. Firstly, Jay and colleagues $(2017 ; 2015)$ used slidescanner obtained images with lower magnification, which make it difficult to distinguish $A \beta$-plaque borders in the analyzed $6 \mathrm{E} 10$ staining. Hence, less condensed $A \beta$-plaques could appear bigger due to loosely attached $A \beta$. Secondly, the authors investigated acute microglia depletion, quantified $A \beta$-plaque size in living animals by congo red administration and in vivo two-photon imaging (Zhao et al., 2017). In our study, we used 6E10 antibody staining in combination with ThioS staining to label the dense core in fixed tissue, which were both shown to correlate well with congo red derivate X-34 dye used in the Trem2 KO studies 
by Wang and co-workers (Crystal et al., 2003; Klunk et al., 2002; Nam et al., 2017; Styren et al., 2000; Wang et al., 2015b; Wang et al., 2016).

\section{PAM function}

In contrast to Trem2 KO, microglial Rab7 KO did not impact PAM recruitment (Figure $27 \mathbf{K}, \mathbf{L}, \mathbf{W}$, $\mathbf{X}$, Figure 28 K, L, W, X). Since Trem2 is upstream of Rab7, we propose that Trem2-mediated DAM formation was not impaired in Rab7 ${ }^{\triangle M G} \times 5 \times F A D$ females and was not compensated by another mechanism. Nonetheless, it remains interesting to quantify the proportion of functional DAM, since these cells are considered a subset of PAM with distinct transcriptional and protein profiles. Even though DAM markers Lpl and IL-17A were analyzed by Olink assay (Figure 30), these data were not sufficient to answer this question, since we analyzed whole hippocampal lysates and other cells of the CNS can also express those markers (Keren-Shaul et al., 2017; Sarma et al., 2009; Tzartos et al., 2008; Wang and Eckel, 2012). Consequently, further analysis is needed to investigate Rab7 $\mathrm{KO}$ on functional alterations of DAM in the Rab7 ${ }^{\triangle M G} \times 5 \times F A D$ mouse model in the future.

\subsection{Role of sex on AB-load in Rab7-mediated degradation in microglia}

In accordance with findings in humans, where $A D$ risk is higher in women than in men and in transgenic AD-mouse lines such as 5xFAD where pathology is more severe in females than in males, the overall $A \beta$-burden and neurotoxicity was higher in females Rab7f/flf $x 5 x F A D$ controls compared to Rab7 $7^{\text {fl/fl }} \times$ 5xFAD males (Bhattacharya et al., 2014; Callahan et al., 2001; Carroll et al., 2010; Johnson et al., 2014; Wang et al., 2003). However, one striking observation in our study was that reduced $A \beta$-plaque numbers and sizes, as well as improved mushroom spine loss, were exclusively found in aged Rab7 ${ }^{\triangle M G} \times 5 \times F A D$ females while no effects in male Rab7 $\triangle M G$ x $5 \times F A D$ were found (Figure 17, Figure 18, Figure 25).

Thus, one possible explanation for these sex-dependent observations in our model could be due to $\mathrm{A} \beta$-dose dependency on Rab7 KO in microglia. As a consequence of the stronger A $\beta$-pathology in females, many studies in rodents are exclusively conducted in this sex (e.g. Eimer and Vassar, 2013; Jawhar et al., 2012; O'Leary et al., 2013). Aside from the sex-effect on A $\beta$-load, there is emerging evidence of sexually dimorphic properties of murine microglia under physiological conditions, and thus most likely also in diseases (Guneykaya et al., 2018; Hanamsagar et al., 2017; Villa et al., 2018). Transcriptome and proteome analyses of WT microglia of 3 weeks and 3 months old animals depicted male microglia to be more reactive, expressing more immune-receptors such as MHCII and TLRs, and bearing higher mortality ergo turnover rates compared to female microglia (Guneykaya et al., 2018; Villa et al., 2018). This was described as developmentally delayed since these features are highly abundant during embryonic development, where female microglia were shown to be highly phagocytic active (Hanamsagar et al., 2017; Schwarz et al., 2012; Stevens et 
al., 2007). This delay was recently also identified in aging: male microglia response was prominently found in TLR2-activation after stroke induction in 15-17 month old males compared to 2-3 month old counterparts and in comparison to females of the same ages (Rahimian et al., 2018). Those microglia sex-dependent features were manifested in adult microglia and could not be changed even upon transplantation into the brains of animals from the opposite sex (Villa et al., 2018). Interestingly, various developmental genes are strongly upregulated in microglia during aging and in AD (Gosselin et al., 2017; Hanamsagar et al., 2017; Krasemann et al., 2017; Rustenhoven et al., 2018). These observations could explain why microglial Rab7 KO in 3 month old animals had no effect in both sexes, but showed effects in females but not in males throughout aging.

Accordingly, a recent study identified transcriptional changes in microglia within the network of AD risk gene ApoE, which were more abundant in aged female mice than in aged males (Kang et al., 2018). In combination with the latter described developmental delay in male microglia, this could also explain findings by meta-analyses of ApoE4 carriers, which described $\mathrm{MCI}$ and $\mathrm{AD}$ risks in women to peak between 65 to 75 years compared to male ApoE4 carriers who showed maximal risk to develop $\mathrm{MCl}$ and $\mathrm{AD}$ at 75 to 85 years of age (Neu et al., 2017). Since ApoE is upstream of Trem2, which in turn is activated before Rab7, this could be a potential explanation why we observed effects $A \beta$-pathology and mild neuroprotection in Rab7 ${ }^{\triangle M G} \times 5 \times F A D$ females at 9 months, whereas we did not detect differences in males of the same age. To elucidate whether male microglial delay was also influencing Rab7-dependent pathways, further experiments would need to be done with older males.

It is worth mentioning that many of the cited studies did not mention the sex or the sex-distribution in their cohorts, therefore it remains difficult to draw conclusions about sex-dependent effects of microglia in A $\mathrm{A}$-pathology and neuronal loss. Noteworthy, the data achieved by ganciclovirmediated ablation of microglia, which did not detect any changes in A $\beta$-load, was conducted in males only (Grathwohl et al., 2009). In contrast, the study which identified significantly more mushroom spines used pools of more or less the same numbers of males and females (Spangenberg et al., 2016). 


\subsection{Conclusion}

This study aimed to investigate whether microglial Rab7 KO could modulate A $\beta$-pathology and neurotoxicity in 5xFAD mice.

We showed that microglial Rab7 KO could reduce the numbers and sizes of A $\beta$-plaques and reduced mushroom spine loss in progressed disease even though the overall amounts of soluble and insoluble $A \beta_{38}, A \beta_{40}$ and $A \beta_{42}$ were not modulated. Interestingly, we detected elevated amounts of chemokine CCL2, which might contribute to infiltration of peripheral myeloid cells or could activate CNS macrophages to compensate for Rab7 KO microglia in 5xFAD mice.

Moreover, Rab7 KO in microglia seems to be more important in females than in males which could be either due to higher $A \beta$-burden in females or due to sex-dependent behavior of microglia.

Together, we conclude that microglial Rab7-dependent degradation of $A \beta$ contributes to $A \beta$-plaque pathology and neurotoxicity in female 5xFAD mice of progressed disease stage. 


\section{Bibliography}

Abraham, C.R., McGraw, W.T., Slot, F., and Yamin, R. (2000). Alpha 1-antichymotrypsin inhibits A beta degradation in vitro and in vivo. Annals of the New York Academy of Sciences 920, 245-248.

Abraham, C.R., Selkoe, D.J., Potter, H., Price, D.L., and Cork, L.C. (1989). Alpha 1antichymotrypsin is present together with the beta-protein in monkey brain amyloid deposits. Neuroscience 32, 715-720.

Ajami, B., Bennett, J.L., Krieger, C., Tetzlaff, W., and Rossi, F.M. (2007). Local self-renewal can sustain CNS microglia maintenance and function throughout adult life. Nature neuroscience 10 , 1538-1543.

Altmann, A., Tian, L., Henderson, V.W., and Greicius, M.D. (2014). Sex modifies the APOE-related risk of developing Alzheimer disease. Annals of neurology 75, 563-573.

Alzheimer, A. (1907). Uber eine eigenartige Erkankung der Hirnrinde. Allgemeine Zeitschrift fur Psychiatrie und Psychisch-gerichtliche Medizin 64:146-8. .

Alzheimer, A., Stelzmann, R.A., Schnitzlein, H.N., and Murtagh, F.R. (1995). An English translation of Alzheimer's 1907 paper, "Uber eine eigenartige Erkankung der Hirnrinde". Clinical anatomy (New York, NY) 8, 429-431.

Amaral, E.P., Riteau, N., Moayeri, M., Maier, N., Mayer-Barber, K.D., Pereira, R.M., Lage, S.L., Kubler, A., Bishai, W.R., D'Império-Lima, M.R., et al. (2018). Lysosomal Cathepsin Release Is Required for NLRP3-Inflammasome Activation by Mycobacterium tuberculosis in Infected Macrophages. Frontiers in immunology 9, 1427.

Armstrong, A., Mattsson, N., Appelqvist, H., Janefjord, C., Sandin, L., Agholme, L., Olsson, B., Svensson, S., Blennow, K., Zetterberg, H., et al. (2014). Lysosomal Network Proteins as Potential Novel CSF Biomarkers for Alzheimer's Disease. Neuromolecular medicine 16, 150-160.

Arnò, B., Grassivaro, F., Rossi, C., Bergamaschi, A., Castiglioni, V., Furlan, R., Greter, M., Favaro, R., Comi, G., Becher, B., et al. (2014). Neural progenitor cells orchestrate microglia migration and positioning into the developing cortex. Nature Communications 5, 5611.

Askew, K., Li, K., Olmos-Alonso, A., Garcia-Moreno, F., Liang, Y., Richardson, P., Tipton, T., Chapman, M.A., Riecken, K., Beccari, S., et al. (2017). Coupled Proliferation and Apoptosis Maintain the Rapid Turnover of Microglia in the Adult Brain. Cell Reports 18, 391-405.

Atagi, Y., Liu, C.C., Painter, M.M., Chen, X.F., Verbeeck, C., Zheng, H., Li, X., Rademakers, R., Kang, S.S., Xu, H., et al. (2015). Apolipoprotein E Is a Ligand for Triggering Receptor Expressed on Myeloid Cells 2 (TREM2). The Journal of biological chemistry 290, 26043-26050.

Ba, L., Chen, X.-H., Chen, Y.-L., Nie, Q., Li, Z.-J., Ding, F.-F., and Zhang, M. (2017). Distinct Rab7related Endosomal\&\#8211;Autophagic\&\#8211;Lysosomal Dysregulation Observed in Cortex and Hippocampus in APPswe/PSEN1dE9 Mouse Model of Alzheimer\&\#39;s Disease. Chinese Medical Journal 130, 2941-2950.

Baik, S.H., Cha, M.Y., Hyun, Y.M., Cho, H., Hamza, B., Kim, D.K., Han, S.H., Choi, H., Kim, K.H., Moon, M., et al. (2014). Migration of neutrophils targeting amyloid plaques in Alzheimer's disease mouse model. Neurobiology of aging 35, 1286-1292. 
Baik, S.H., Kang, S., Son, S.M., and Mook-Jung, I. (2016). Microglia contributes to plaque growth by cell death due to uptake of amyloid beta in the brain of Alzheimer's disease mouse model. Glia $64,2274-2290$.

Baker, M., Mackenzie, I.R., Pickering-Brown, S.M., Gass, J., Rademakers, R., Lindholm, C., Snowden, J., Adamson, J., Sadovnick, A.D., Rollinson, S., et al. (2006). Mutations in progranulin cause tau-negative frontotemporal dementia linked to chromosome 17. Nature 442, 916.

Bales, K.R., Verina, T., Dodel, R.C., Du, Y., Altstiel, L., Bender, M., Hyslop, P., Johnstone, E.M., Little, S.P., Cummins, D.J., et al. (1997). Lack of apolipoprotein E dramatically reduces amyloidbeta peptide deposition. Nature genetics 17 .

Baranger, K., Bonnet, A.E., Girard, S.D., Paumier, J.-M., García-González, L., Elmanaa, W., Bernard, A., Charrat, E., Stephan, D., Bauer, C., et al. (2017). MT5-MMP Promotes Alzheimer's Pathogenesis in the Frontal Cortex of 5xFAD Mice and APP Trafficking in vitro. Frontiers in Molecular Neuroscience 9.

Baroja-Mazo, A., Martin-Sanchez, F., Gomez, A.I., Martinez, C.M., Amores-Iniesta, J., Compan, V., Barbera-Cremades, M., Yague, J., Ruiz-Ortiz, E., Anton, J., et al. (2014). The NLRP3 inflammasome is released as a particulate danger signal that amplifies the inflammatory response. Nature immunology $15,738-748$.

Bauernfeind, F.G., Horvath, G., Stutz, A., Alnemri, E.S., MacDonald, K., Speert, D., FernandesAlnemri, T., Wu, J., Monks, B.G., Fitzgerald, K.A., et al. (2009). Cutting edge: NF-kappaB activating pattern recognition and cytokine receptors license NLRP3 inflammasome activation by regulating NLRP3 expression. Journal of immunology (Baltimore, Md : 1950) 183, 787-791.

Bazan, J.F., Bacon, K.B., Hardiman, G., Wang, W., Soo, K., Rossi, D., Greaves, D.R., Zlotnik, A., and Schall, T.J. (1997). A new class of membrane-bound chemokine with a CX3C motif. Nature $385,640-644$.

Bechmann, I., Priller, J., Kovac, A., Bontert, M., Wehner, T., Klett, F.F., Bohsung, J., Stuschke, M., Dirnagl, U., and Nitsch, R. (2001). Immune surveillance of mouse brain perivascular spaces by blood-borne macrophages. The European journal of neuroscience 14, 1651-1658.

Becker-Pauly, C., and Pietrzik, C.U. (2016). The Metalloprotease Meprin $\beta$ Is an Alternative $\beta$ Secretase of APP. Frontiers in Molecular Neuroscience 9, 159.

Beeg, M., Stravalaci, M., Romeo, M., Carra, A.D., Cagnotto, A., Rossi, A., Diomede, L., Salmona, M., and Gobbi, M. (2016). Clusterin Binds to Abeta1-42 Oligomers with High Affinity and Interferes with Peptide Aggregation by Inhibiting Primary and Secondary Nucleation. The Journal of biological chemistry 291, 6958-6966.

Bell, R.D., Sagare, A.P., Friedman, A.E., Bedi, G.S., Holtzman, D.M., Deane, R., and Zlokovic, B.V. (2007). Transport pathways for clearance of human Alzheimer's amyloid beta-peptide and apolipoproteins $E$ and $J$ in the mouse central nervous system. Journal of cerebral blood flow and metabolism : official journal of the International Society of Cerebral Blood Flow and Metabolism 27, 909-918.

Bell, R.D., Winkler, E.A., Singh, I., Sagare, A.P., Deane, R., Wu, Z., Holtzman, D.M., Betsholtz, C., Armulik, A., Sallstrom, J., et al. (2012). Apolipoprotein E controls cerebrovascular integrity via cyclophilin A. Nature 485, 512-516.

Bennett, M.L., Bennett, F.C., Liddelow, S.A., Ajami, B., Zamanian, J.L., Fernhoff, N.B., Mulinyawe, S.B., Bohlen, C.J., Adil, A., Tucker, A., et al. (2016). New tools for studying microglia in the mouse and human CNS. Proceedings of the National Academy of Sciences 113, E1738-E1746. 
Bertram, L., McQueen, M.B., Mullin, K., Blacker, D., and Tanzi, R.E. (2007). Systematic metaanalyses of Alzheimer disease genetic association studies: the AlzGene database. Nature genetics $39,17-23$.

Bhattacharya, M., Ojha, N., Solanki, S., Mukhopadhyay, C.K., Madan, R., Patel, N., Krishnamurthy, G., Kumar, S., Basu, S.K., and Mukhopadhyay, A. (2006). IL-6 and IL-12 specifically regulate the expression of Rab5 and Rab7 via distinct signaling pathways. The EMBO journal 25, 2878-2888.

Bhattacharya, S., Haertel, C., Maelicke, A., and Montag, D. (2014). Galantamine slows down plaque formation and behavioral decline in the 5XFAD mouse model of Alzheimer's disease. PloS one 9, e89454.

Biere, A.L., Ostaszewski, B., Stimson, E.R., Hyman, B.T., Maggio, J.E., and Selkoe, D.J. (1996). Amyloid beta-peptide is transported on lipoproteins and albumin in human plasma. The Journal of biological chemistry 271, 32916-32922.

Bobkova, N.V., Garbuz, D.G., Nesterova, I., Medvinskaya, N., Samokhin, A., Alexandrova, I., Yashin, V., Karpov, V., Kukharsky, M.S., Ninkina, N.N., et al. (2014). Therapeutic effect of exogenous hsp70 in mouse models of Alzheimer's disease. Journal of Alzheimer's disease : JAD $38,425-435$.

Boisvert, M.M., Erikson, G.A., Shokhirev, M.N., and Allen, N.J. (2018). The Aging Astrocyte Transcriptome from Multiple Regions of the Mouse Brain. Cell Reports 22, 269-285.

Boland, B., Kumar, A., Lee, S., Platt, F.M., Wegiel, J., Yu, W.H., and Nixon, R.A. (2008). Autophagy Induction and Autophagosome Clearance in Neurons: Relationship to Autophagic Pathology in Alzheimer's Disease. The Journal of neuroscience : the official journal of the Society for Neuroscience 28, 6926-6937.

Boland, B., Yu, W.H., Corti, O., Mollereau, B., Henriques, A., Bezard, E., Pastores, G.M., Rubinsztein, D.C., Nixon, R.A., Duchen, M.R., et al. (2018). Promoting the clearance of neurotoxic proteins in neurodegenerative disorders of ageing. Nature reviews Drug discovery 17, 660-688.

Bolmont, T., Haiss, F., Eicke, D., Radde, R., Mathis, C.A., Klunk, W.E., Kohsaka, S., Jucker, M., and Calhoun, M.E. (2008). Dynamics of the microglial/amyloid interaction indicate a role in plaque maintenance. J Neurosci 28, 4283-4292.

Borchelt, D.R., Ratovitski, T., van Lare, J., Lee, M.K., Gonzales, V., Jenkins, N.A., Copeland, N.G., Price, D.L., and Sisodia, S.S. (1997). Accelerated amyloid deposition in the brains of transgenic mice coexpressing mutant presenilin 1 and amyloid precursor proteins. Neuron 19, 939-945.

Boulanger, L.M. (2009). Immune proteins in brain development and synaptic plasticity. Neuron 64 , 93-109.

Boza-Serrano, A., Yang, Y., Paulus, A., and Deierborg, T. (2018). Innate immune alterations are elicited in microglial cells before plaque deposition in the Alzheimer's disease mouse model 5xFAD. Scientific reports 8, 1550.

Braak, H., and Braak, E. (1991). Neuropathological stageing of Alzheimer-related changes. Acta neuropathologica 82, 239-259.

Brouwers, N., Van Cauwenberghe, C., Engelborghs, S., Lambert, J.C., Bettens, K., Le Bastard, N., Pasquier, F., Montoya, A.G., Peeters, K., Mattheijssens, M., et al. (2012). Alzheimer risk associated with a copy number variation in the complement receptor 1 increasing $C 3 \mathrm{~b} / \mathrm{C} 4 \mathrm{~b}$ binding sites.

Molecular psychiatry 17, 223-233. 
Bruttger, J., Karram, K., Wortge, S., Regen, T., Marini, F., Hoppmann, N., Klein, M., Blank, T., Yona, S., Wolf, Y., et al. (2015). Genetic Cell Ablation Reveals Clusters of Local Self-Renewing Microglia in the Mammalian Central Nervous System. Immunity 43, 92-106.

Bucci, C., Thomsen, P., Nicoziani, P., McCarthy, J., Deurs, B.v., and Pfeffer, S.R. (2000). Rab7: A Key to Lysosome Biogenesis. Molecular biology of the cell 11, 467-480.

Butovsky, O., Jedrychowski, M.P., Moore, C.S., Cialic, R., Lanser, A.J., Gabriely, G., Koeglsperger, T., Dake, B., Wu, P.M., Doykan, C.E., et al. (2014). Identification of a unique TGF-beta-dependent molecular and functional signature in microglia. Nature neuroscience 17, 131-143.

Cabrejo, L., Guyant-Marechal, L., Laquerriere, A., Vercelletto, M., De la Fourniere, F., ThomasAnterion, C., Verny, C., Letournel, F., Pasquier, F., Vital, A., et al. (2006). Phenotype associated with APP duplication in five families. Brain : a journal of neurology 129, 2966-2976.

Caccamo, A., Majumder, S., Richardson, A., Strong, R., and Oddo, S. (2010). Molecular interplay between mammalian target of rapamycin (mTOR), amyloid-beta, and Tau: effects on cognitive impairments. The Journal of biological chemistry 285, 13107-13120.

Cai, H., Wang, Y., McCarthy, D., Wen, H., Borchelt, D.R., Price, D.L., and Wong, P.C. (2001). BACE1 is the major beta-secretase for generation of Abeta peptides by neurons. Nature neuroscience 4, 233-234.

Cai, Q., Lu, L., Tian, J.H., Zhu, Y.B., Qiao, H., and Sheng, Z.H. (2010). Snapin-regulated late endosomal transport is critical for efficient autophagy-lysosomal function in neurons. Neuron 68, 7386.

Callahan, M.J., Lipinski, W.J., Bian, F., Durham, R.A., Pack, A., and Walker, L.C. (2001).

Augmented Senile Plaque Load in Aged Female $\beta$-Amyloid Precursor Protein-Transgenic Mice. The American journal of pathology 158, 1173-1177.

Cantalupo, G., Alifano, P., Roberti, V., Bruni, C.B., and Bucci, C. (2001). Rab-interacting lysosomal protein (RILP): the Rab7 effector required for transport to lysosomes. The EMBO journal 20, 683693.

Carroll, J.C., Rosario, E.R., Kreimer, S., Villamagna, A., Gentzschein, E., Stanczyk, F.Z., and Pike, C.J. (2010). Sex differences in $\beta$-amyloid accumulation in 3xTg-AD mice: Role of neonatal sex steroid hormone exposure. Brain research 1366, 233-245.

Cascella, R., Conti, S., Tatini, F., Evangelisti, E., Scartabelli, T., Casamenti, F., Wilson, M.R., Chiti, F., and Cecchi, C. (2013). Extracellular chaperones prevent Abeta42-induced toxicity in rat brains. Biochimica et biophysica acta 1832, 1217-1226.

Castano, E.M., Prelli, F., Wisniewski, T., Golabek, A., Kumar, R.A., Soto, C., and Frangione, B. (1995). Fibrillogenesis in Alzheimer's disease of amyloid beta peptides and apolipoprotein E. The Biochemical journal 306 ( Pt 2), 599-604.

Castellano, J.M., Kim, J., Stewart, F.R., Jiang, H., DeMattos, R.B., Patterson, B.W., Fagan, A.M., Morris, J.C., Mawuenyega, K.G., Cruchaga, C., et al. (2011). Human apoE isoforms differentially regulate brain amyloid-beta peptide clearance. Science translational medicine 3, 89ra57.

Cataldo, A.M., Mathews, P.M., Boiteau, A.B., Hassinger, L.C., Peterhoff, C.M., Jiang, Y., Mullaney, K., Neve, R.L., Gruenberg, J., and Nixon, R.A. (2008). Down syndrome fibroblast model of Alzheimer-related endosome pathology: accelerated endocytosis promotes late endocytic defects. The American journal of pathology 173, 370-384. 
Cataldo, A.M., and Nixon, R.A. (1990). Enzymatically active lysosomal proteases are associated with amyloid deposits in Alzheimer brain. Proceedings of the National Academy of Sciences of the United States of America 87, 3861-3865.

Cataldo, A.M., Peterhoff, C.M., Troncoso, J.C., Gomez-Isla, T., Hyman, B.T., and Nixon, R.A. (2000). Endocytic pathway abnormalities precede amyloid beta deposition in sporadic Alzheimer's disease and Down syndrome: differential effects of APOE genotype and presenilin mutations. The American journal of pathology 157, 277-286.

Chan, G., White, C.C., Winn, P.A., Cimpean, M., Replogle, J.M., Glick, L.R., Cuerdon, N.E., Ryan, K.J., Johnson, K.A., Schneider, J.A., et al. (2015). CD33 modulates TREM2: convergence of Alzheimer loci. Nature neuroscience 18, 1556.

Chishti, M.A., Yang, D.S., Janus, C., Phinney, A.L., Horne, P., Pearson, J., Strome, R., Zuker, N., Loukides, J., French, J., et al. (2001). Early-onset amyloid deposition and cognitive deficits in transgenic mice expressing a double mutant form of amyloid precursor protein 695 . The Journal of biological chemistry 276, 21562-21570.

Cho, M.-H., Cho, K., Kang, H.-J., Jeon, E.-Y., Kim, H.-S., Kwon, H.-J., Kim, H.-M., Kim, D.-H., and Yoon, S.-Y. (2014). Autophagy in microglia degrades extracellular $\beta$-amyloid fibrils and regulates the NLRP3 inflammasome. Autophagy 10, 1761-1775.

Chung, H., Brazil, M.I., Soe, T.T., and Maxfield, F.R. (1999). Uptake, degradation, and release of fibrillar and soluble forms of Alzheimer's amyloid beta-peptide by microglial cells. The Journal of biological chemistry 274, 32301-32308.

Condello, C., Yuan, P., Schain, A., and Grutzendler, J. (2015). Microglia constitute a barrier that prevents neurotoxic protofibrillar A 342 hotspots around plaques. Nature Communications 6, 6176 .

Crowe, S.E., and Ellis-Davies, G.C.R. (2014). Spine pruning in 5xFAD mice starts on basal dendrites of layer 5 pyramidal neurons. Brain structure \& function 219, 571-580.

Cruts, M., Gijselinck, I., van der Zee, J., Engelborghs, S., Wils, H., Pirici, D., Rademakers, R., Vandenberghe, R., Dermaut, B., Martin, J.-J., et al. (2006). Null mutations in progranulin cause ubiquitin-positive frontotemporal dementia linked to chromosome 17q21. Nature 442, 920.

Crystal, A.S., Giasson, B.I., Crowe, A., Kung, M.P., Zhuang, Z.P., Trojanowski, J.Q., and Lee, V.M. (2003). A comparison of amyloid fibrillogenesis using the novel fluorescent compound K114. Journal of neurochemistry $86,1359-1368$.

Czirr, E., Castello, N.A., Mosher, K.I., Castellano, J.M., Hinkson, I.V., Lucin, K.M., Baeza-Raja, B., Ryu, J.K., Li, L., Farina, S.N., et al. (2017). Microglial complement receptor 3 regulates brain Abeta levels through secreted proteolytic activity. The Journal of experimental medicine 214, 1081-1092.

D'Andrea, M.R., Nagele, R.G., Wang, H.Y., and Lee, D.H. (2002). Consistent immunohistochemical detection of intracellular beta-amyloid42 in pyramidal neurons of Alzheimer's disease entorhinal cortex. Neuroscience letters 333, 163-166.

Dagher, N.N., Najafi, A.R., Kayala, K.M., Elmore, M.R., White, T.E., Medeiros, R., West, B.L., and Green, K.N. (2015). Colony-stimulating factor 1 receptor inhibition prevents microglial plaque association and improves cognition in 3xTg-AD mice. Journal of neuroinflammation 12, 139.

Damani, M.R., Zhao, L., Fontainhas, A.M., Amaral, J., Fariss, R.N., and Wong, W.T. (2011). Agerelated alterations in the dynamic behavior of microglia. Aging cell 10, 263-276. 
Daria, A., Colombo, A., Llovera, G., Hampel, H., Willem, M., Liesz, A., Haass, C., and Tahirovic, S. (2017). Young microglia restore amyloid plaque clearance of aged microglia. The EMBO journal 36 , 583-603.

Das, U., Wang, L., Ganguly, A., Saikia, J.M., Wagner, S.L., Koo, E.H., and Roy, S. (2015). Visualizing APP and BACE-1 approximation in neurons yields insight into the amyloidogenic pathway. Nature neuroscience 19, 55.

Davalos, D., Grutzendler, J., Yang, G., Kim, J.V., Zuo, Y., Jung, S., Littman, D.R., Dustin, M.L., and Gan, W.B. (2005). ATP mediates rapid microglial response to local brain injury in vivo. Nature neuroscience 8, 752-758.

Davies, D.S., Ma, J., Jegathees, T., and Goldsbury, C. (2017). Microglia show altered morphology and reduced arborization in human brain during aging and Alzheimer's disease. Brain Pathol 27, 795-808.

De Jonghe, C., Cruts, M., Rogaeva, E.A., Tysoe, C., Singleton, A., Vanderstichele, H., Meschino, W., Dermaut, B., Vanderhoeven, I., Backhovens, H., et al. (1999). Aberrant splicing in the presenilin-1 intron 4 mutation causes presenile Alzheimer's disease by increased Abeta42 secretion. Human molecular genetics 8, 1529-1540.

De Jonghe, C., Esselens, C., Kumar-Singh, S., Craessaerts, K., Serneels, S., Checler, F., Annaert, W., Van Broeckhoven, C., and De Strooper, B. (2001). Pathogenic APP mutations near the gammasecretase cleavage site differentially affect Abeta secretion and APP C-terminal fragment stability. Human molecular genetics 10, 1665-1671.

De Strooper, B., Iwatsubo, T., and Wolfe, M.S. (2012). Presenilins and gamma-secretase: structure, function, and role in Alzheimer Disease. Cold Spring Harbor perspectives in medicine 2, a006304.

Deane, R., Sagare, A., Hamm, K., Parisi, M., Lane, S., Finn, M.B., Holtzman, D.M., and Zlokovic, B.V. (2008). apoE isoform - specific disruption of amyloid $\beta$ peptide clearance from mouse brain. The Journal of clinical investigation 118.

Dubois, B., Feldman, H.H., Jacova, C., Dekosky, S.T., Barberger-Gateau, P., Cummings, J., Delacourte, A., Galasko, D., Gauthier, S., Jicha, G., et al. (2007). Research criteria for the diagnosis of Alzheimer's disease: revising the NINCDS-ADRDA criteria. The Lancet Neurology 6, 734-746.

Eckman, C.B., Mehta, N.D., Crook, R., Perez-tur, J., Prihar, G., Pfeiffer, E., Graff-Radford, N., Hinder, P., Yager, D., Zenk, B., et al. (1997). A new pathogenic mutation in the APP gene (I716V) increases the relative proportion of $A$ beta 42(43). Human molecular genetics 6, 2087-2089.

Edinger, A.L., Cinalli, R.M., and Thompson, C.B. (2003). Rab7 prevents growth factor-independent survival by inhibiting cell-autonomous nutrient transporter expression. Developmental cell 5, 571582.

Eimer, W., and Vassar, R. (2013). Neuron loss in the 5XFAD mouse model of Alzheimer's disease correlates with intraneuronal A 42 accumulation and Caspase-3 activation, Vol 8.

El Khoury, J., Toft, M., Hickman, S.E., Means, T.K., Terada, K., Geula, C., and Luster, A.D. (2007). Ccr2 deficiency impairs microglial accumulation and accelerates progression of Alzheimer-like disease. Nature medicine 13, 432-438.

Fabriek, B.O., Van Haastert, E.S., Galea, I., Polfliet, M.M., Dopp, E.D., Van Den Heuvel, M.M., Van Den Berg, T.K., De Groot, C.J., Van Der Valk, P., and Dijkstra, C.D. (2005). CD163-positive perivascular macrophages in the human CNS express molecules for antigen recognition and presentation. Glia 51, 297-305. 
Farrer, L.A., Cupples, L.A., Haines, J.L., Hyman, B., Kukull, W.A., Mayeux, R., Myers, R.H., Pericak-Vance, M.A., Risch, N., and van Duijn, C.M. (1997). Effects of age, sex, and ethnicity on the association between apolipoprotein $\mathrm{E}$ genotype and Alzheimer disease. A meta-analysis. APOE and Alzheimer Disease Meta Analysis Consortium. Jama 278, 1349-1356.

Feng, Y., Press, B., and Wandinger-Ness, A. (1995). Rab 7: an important regulator of late endocytic membrane traffic. The Journal of cell biology 131, 1435-1452.

Flowers, A., Bell-Temin, H., Jalloh, A., Stevens, S.M., and Bickford, P.C. (2017). Proteomic analysis of aged microglia: shifts in transcription, bioenergetics, and nutrient response. Journal of neuroinflammation 14, 96 .

Fonseca, M.I., Chu, S.-H., Hernandez, M.X., Fang, M.J., Modarresi, L., Selvan, P., MacGregor, G.R., and Tenner, A.J. (2017). Cell-specific deletion of C1qa identifies microglia as the dominant source of C1q in mouse brain. Journal of neuroinflammation 14, 48.

Fonseca, M.I., Zhou, J., Botto, M., and Tenner, A.J. (2004). Absence of C1q Leads to Less Neuropathology in Transgenic Mouse Models of Alzheimer's Disease. The Journal of Neuroscience 24, 6457-6465.

Frackowiak, J., Wisniewski, H.M., Wegiel, J., Merz, G.S., Iqbal, K., and Wang, K.C. (1992). Ultrastructure of the microglia that phagocytose amyloid and the microglia that produce betaamyloid fibrils. Acta neuropathologica 84, 225-233.

Franceschi, C., Bonafe, M., Valensin, S., Olivieri, F., De Luca, M., Ottaviani, E., and De Benedictis, G. (2000). Inflamm-aging. An evolutionary perspective on immunosenescence. Annals of the New York Academy of Sciences 908, 244-254.

Franchi, L., Eigenbrod, T., and Nunez, G. (2009). Cutting edge: TNF-alpha mediates sensitization to ATP and silica via the NLRP3 inflammasome in the absence of microbial stimulation. Journal of immunology (Baltimore, Md : 1950) 183, 792-796.

Franklin, B.S., Bossaller, L., De Nardo, D., Ratter, J.M., Stutz, A., Engels, G., Brenker, C., Nordhoff, M., Mirandola, S.R., Al-Amoudi, A., et al. (2014). The adaptor ASC has extracellular and 'prionoid' activities that propagate inflammation. Nature immunology 15, 727-737.

Fraser, P.E., Nguyen, J.T., McLachlan, D.R., Abraham, C.R., and Kirschner, D.A. (1993). a1Antichymotrypsin Binding to Alzheimer A $\beta$ Peptides Is Sequence Specific and Induces Fibril Disaggregation In Vitro. Journal of neurochemistry 61, 298-305.

Friedman, B.A., Srinivasan, K., Ayalon, G., Meilandt, W.J., Lin, H., Huntley, M.A., Cao, Y., Lee, S.H., Haddick, P.C.G., Ngu, H., et al. (2018). Diverse Brain Myeloid Expression Profiles Reveal Distinct Microglial Activation States and Aspects of Alzheimer's Disease Not Evident in Mouse Models. Cell Rep 22, 832-847.

Frozza, R.L., Lourenco, M.V., and De Felice, F.G. (2018). Challenges for Alzheimer's Disease Therapy: Insights from Novel Mechanisms Beyond Memory Defects. Frontiers in neuroscience 12, 37.

Funato, H., Yoshimura, M., Yamazaki, T., Saido, T.C., Ito, Y., Yokofujita, J., Okeda, R., and Ihara, Y. (1998). Astrocytes containing amyloid beta-protein (Abeta)-positive granules are associated with Abeta40-positive diffuse plaques in the aged human brain. The American journal of pathology 152 , 983-992. 
Galvin, J.E., Fagan, A.M., Holtzman, D.M., Mintun, M.A., and Morris, J.C. (2010). Relationship of dementia screening tests with biomarkers of Alzheimer's disease. Brain : a journal of neurology 133, 3290-3300.

Games, D., Adams, D., Alessandrini, R., Barbour, R., Berthelette, P., Blackwell, C., Carr, T., Clemens, J., Donaldson, T., Gillespie, F., et al. (1995). Alzheimer-type neuropathology in transgenic mice overexpressing V717F beta-amyloid precursor protein. Nature 373, 523-527.

Gautier, E.L., Shay, T., Miller, J., Greter, M., Jakubzick, C., Ivanov, S., Helft, J., Chow, A., Elpek, K.G., Gordonov, S., et al. (2012). Gene-expression profiles and transcriptional regulatory pathways that underlie the identity and diversity of mouse tissue macrophages. Nature immunology 13,1118 1128.

Ginhoux, F., Greter, M., Leboeuf, M., Nandi, S., See, P., Gokhan, S., Mehler, M.F., Conway, S.J., Ng, L.G., Stanley, E.R., et al. (2010). Fate mapping analysis reveals that adult microglia derive from primitive macrophages. Science 330, 841-845.

Ginhoux, F., Lim, S., Hoeffel, G., Low, D., and Huber, T. (2013). Origin and differentiation of microglia. Frontiers in cellular neuroscience 7.

Ginsberg, S.D., Alldred, M.J., Counts, S.E., Cataldo, A.M., Neve, R.L., Jiang, Y., Wuu, J., Chao, M.V., Mufson, E.J., Nixon, R.A., et al. (2010a). Microarray analysis of hippocampal CA1 neurons implicates early endosomal dysfunction during Alzheimer's disease progression. Biological psychiatry $68,885-893$.

Ginsberg, S.D., Mufson, E.J., Alldred, M.J., Counts, S.E., Wuu, J., Nixon, R.A., and Che, S. (2011). Upregulation of select rab GTPases in cholinergic basal forebrain neurons in mild cognitive impairment and Alzheimer's disease. Journal of chemical neuroanatomy 42, 102-110.

Ginsberg, S.D., Mufson, E.J., Counts, S.E., Wuu, J., Alldred, M.J., Nixon, R.A., and Che, S. (2010b). Regional selectivity of rab5 and rab7 protein upregulation in mild cognitive impairment and Alzheimer's disease. Journal of Alzheimer's disease : JAD 22, 631-639.

Glynn-Servedio, B.E., and Ranola, T.S. (2017). AChE Inhibitors and NMDA Receptor Antagonists in Advanced Alzheimer's Disease. The Consultant pharmacist : the journal of the American Society of Consultant Pharmacists 32, 511-518.

Goate, A., Chartier-Harlin, M.C., Mullan, M., Brown, J., Crawford, F., Fidani, L., Giuffra, L., Haynes, A., Irving, N., James, L., et al. (1991). Segregation of a missense mutation in the amyloid precursor protein gene with familial Alzheimer's disease. Nature 349, 704-706.

Goedert, M. (2015). Alzheimer's and Parkinson's diseases: The prion concept in relation to assembled $A \beta$, tau, and $\alpha$-synuclein. Science 349.

Goedert, M., Klug, A., and Crowther, R.A. (2006). Tau protein, the paired helical filament and Alzheimer's disease. Journal of Alzheimer's disease : JAD 9, 195-207.

Goedert, M., Wischik, C.M., Crowther, R.A., Walker, J.E., and Klug, A. (1988). Cloning and sequencing of the CDNA encoding a core protein of the paired helical filament of Alzheimer disease: identification as the microtubule-associated protein tau. Proceedings of the National Academy of Sciences of the United States of America 85, 4051-4055.

Goldmann, T., Jordão, M.J.C., Wieghofer, P., Prutek, F., Hagemeyer, N., Frenzel, K., Staszewski, O., Kierdorf, K., Amann, L., Krueger, M., et al. (2016). Origin, fate and dynamics of macrophages at CNS interfaces. Nature immunology 17, 797-805. 
Gomez-Arboledas, A., Davila, J.C., Sanchez-Mejias, E., Navarro, V., Nunez-Diaz, C., SanchezVaro, R., Sanchez-Mico, M.V., Trujillo-Estrada, L., Fernandez-Valenzuela, J.J., Vizuete, M., et al. (2018). Phagocytic clearance of presynaptic dystrophies by reactive astrocytes in Alzheimer's disease. Glia 66, 637-653.

Gosselin, D., Skola, D., Coufal, N.G., Holtman, I.R., Schlachetzki, J.C.M., Sajti, E., Jaeger, B.N., O'Connor, C., Fitzpatrick, C., Pasillas, M.P., et al. (2017). An environment-dependent transcriptional network specifies human microglia identity. Science 356.

Gouras, G.K., Tsai, J., Naslund, J., Vincent, B., Edgar, M., Checler, F., Greenfield, J.P., Haroutunian, V., Buxbaum, J.D., Xu, H., et al. (2000). Intraneuronal Abeta42 accumulation in human brain. The American journal of pathology 156, 15-20.

Gouw, A.A., Seewann, A., Vrenken, H., van der Flier, W.M., Rozemuller, J.M., Barkhof, F., Scheltens, P., and Geurts, J.J.G. (2008). Heterogeneity of white matter hyperintensities in Alzheimer's disease: post-mortem quantitative MRI and neuropathology. Brain : a journal of neurology 131, 3286-3298.

Grathwohl, S.A., Kalin, R.E., Bolmont, T., Prokop, S., Winkelmann, G., Kaeser, S.A., Odenthal, J., Radde, R., Eldh, T., Gandy, S., et al. (2009). Formation and maintenance of Alzheimer's disease beta-amyloid plaques in the absence of microglia. Nature neuroscience 12, 1361-1363.

Gregori, L., Fuchs, C., Figueiredo-Pereira, M.E., Van Nostrand, W.E., and Goldgaber, D. (1995). Amyloid beta-protein inhibits ubiquitin-dependent protein degradation in vitro. The Journal of biological chemistry 270, 19702-19708.

Griffin, R., Nally, R., Nolan, Y., McCartney, Y., Linden, J., and Lynch, M.A. (2006). The age-related attenuation in long-term potentiation is associated with microglial activation. Journal of neurochemistry 99, 1263-1272.

Grutzendler, J., Kasthuri, N., and Gan, W.B. (2002). Long-term dendritic spine stability in the adult cortex. Nature 420, 812-816.

Guerreiro, R., Wojtas, A., Bras, J., Carrasquillo, M., Rogaeva, E., Majounie, E., Cruchaga, C., Sassi, C., Kauwe, J.S., Younkin, S., et al. (2013). TREM2 variants in Alzheimer's disease. The New England journal of medicine 368, 117-127.

Guneykaya, D., Ivanov, A., Hernandez, D.P., Haage, V., Wojtas, B., Meyer, N., Maricos, M., Jordan, P., Buonfiglioli, A., Gielniewski, B., et al. (2018). Transcriptional and Translational Differences of Microglia from Male and Female Brains. Cell Rep 24, 2773-2783.e2776.

Gutierrez, M.G., Munafo, D.B., Beron, W., and Colombo, M.I. (2004). Rab7 is required for the normal progression of the autophagic pathway in mammalian cells. Journal of cell science 117 , 2687-2697.

Haass, C., Schlossmacher, M.G., Hung, A.Y., Vigo-Pelfrey, C., Mellon, A., Ostaszewski, B.L., Lieberburg, I., Koo, E.H., Schenk, D., Teplow, D.B., et al. (1992). Amyloid beta-peptide is produced by cultured cells during normal metabolism. Nature 359, 322-325.

Halle, A., Hornung, V., Petzold, G.C., Stewart, C.R., Monks, B.G., Reinheckel, T., Fitzgerald, K.A., Latz, E., Moore, K.J., and Golenbock, D.T. (2008). The NALP3 inflammasome is involved in the innate immune response to amyloid- $\beta$. Nature immunology $9,857$.

Hanamsagar, R., Alter, M.D., Block, C.S., Sullivan, H., Bolton, J.L., and Bilbo, S.D. (2017). Generation of a microglial developmental index in mice and in humans reveals a sex difference in maturation and immune reactivity. Glia 65, 1504-1520. 
Hara, T., Nakamura, K., Matsui, M., Yamamoto, A., Nakahara, Y., Suzuki-Migishima, R., Yokoyama, M., Mishima, K., Saito, I., Okano, H., et al. (2006). Suppression of basal autophagy in neural cells causes neurodegenerative disease in mice. Nature 441, 885-889.

Hardy, J., and Higgins, G. (1992). Alzheimer's disease: the amyloid cascade hypothesis. Science 256, 184-185.

Hashimoto, D., Chow, A., Noizat, C., Teo, P., Beasley, M.B., Leboeuf, M., Becker, C.D., See, P., Price, J., Lucas, D., et al. (2013). Tissue-resident macrophages self-maintain locally throughout adult life with minimal contribution from circulating monocytes. Immunity 38, 792-804.

Hawkes, C.A., and McLaurin, J. (2009). Selective targeting of perivascular macrophages for clearance of $\beta$-amyloid in cerebral amyloid angiopathy. Proceedings of the National Academy of Sciences of the United States of America 106, 1261-1266.

Hebert, L.E., Scherr, P.A., McCann, J.J., Beckett, L.A., and Evans, D.A. (2001). Is the risk of developing Alzheimer's disease greater for women than for men? American journal of epidemiology 153, $132-136$.

Hendriks, L., van Duijn, C.M., Cras, P., Cruts, M., Van Hul, W., van Harskamp, F., Warren, A., McInnis, M.G., Antonarakis, S.E., Martin, J.J., et al. (1992). Presenile dementia and cerebral haemorrhage linked to a mutation at codon 692 of the beta-amyloid precursor protein gene. Nature genetics $1,218-221$.

Heneka, M.T., Kummer, M.P., Stutz, A., Delekate, A., Schwartz, S., Vieira-Saecker, A., Griep, A., Axt, D., Remus, A., Tzeng, T.-C., et al. (2012). NLRP3 is activated in Alzheimer's disease and contributes to pathology in APP/PS1 mice. Nature 493, 674.

Henry, C.J., Huang, Y., Wynne, A.M., and Godbout, J.P. (2009). Peripheral lipopolysaccharide (LPS) challenge promotes microglial hyperactivity in aged mice that is associated with exaggerated induction of both pro-inflammatory IL-1 beta and anti-inflammatory IL-10 cytokines. Brain, behavior, and immunity $23,309-317$.

Heppner, F.L., Ransohoff, R.M., and Becher, B. (2015). Immune attack: the role of inflammation in Alzheimer disease. Nature Reviews Neuroscience 16, 358.

Herisson, F., Frodermann, V., Courties, G., Rohde, D., Sun, Y., Vandoorne, K., Wojtkiewicz, G.R., Masson, G.S., Vinegoni, C., Kim, J., et al. (2018). Direct vascular channels connect skull bone marrow and the brain surface enabling myeloid cell migration. Nature neuroscience.

Hernandez-Guillamon, M., Mawhirt, S., Fossati, S., Blais, S., Pares, M., Penalba, A., Boada, M., Couraud, P.-O., Neubert, T.A., Montaner, J., et al. (2010). Matrix Metalloproteinase 2 (MMP-2) Degrades Soluble Vasculotropic Amyloid- $\beta$ E22Q and L34V Mutants, Delaying Their Toxicity for Human Brain Microvascular Endothelial Cells. The Journal of biological chemistry 285, 2714427158.

Hickman, S.E., Allison, E.K., and El Khoury, J. (2008). Microglial dysfunction and defective betaamyloid clearance pathways in aging Alzheimer's disease mice. J Neurosci 28, 8354-8360.

Hochgrafe, K., Sydow, A., and Mandelkow, E.M. (2013). Regulatable transgenic mouse models of Alzheimer disease: onset, reversibility and spreading of Tau pathology. The FEBS journal 280 , 4371-4381.

Hollingworth, P., Harold, D., Sims, R., Gerrish, A., Lambert, J.C., Carrasquillo, M.M., Abraham, R., Hamshere, M.L., Pahwa, J.S., Moskvina, V., et al. (2011). Common variants at ABCA7, 
MS4A6A/MS4A4E, EPHA1, CD33 and CD2AP are associated with Alzheimer's disease. Nature genetics $43,429-435$.

Holtman, I.R., Raj, D.D., Miller, J.A., Schaafsma, W., Yin, Z., Brouwer, N., Wes, P.D., Moller, T., Orre, M., Kamphuis, W., et al. (2015). Induction of a common microglia gene expression signature by aging and neurodegenerative conditions: a co-expression meta-analysis. Acta neuropathologica communications 3,31 .

Holtzman, D.M., Bales, K.R., Tenkova, T., Fagan, A.M., Parsadanian, M., Sartorius, L.J., Mackey, B., Olney, J., McKeel, D., Wozniak, D., et al. (2000a). Apolipoprotein E isoform-dependent amyloid deposition and neuritic degeneration in a mouse model of Alzheimer's disease. Proceedings of the National Academy of Sciences of the United States of America 97, 2892-2897.

Holtzman, D.M., Fagan, A.M., Mackey, B., Tenkova, T., Sartorius, L., Paul, S.M., Bales, K., Ashe, K.H., Irizarry, M.C., and Hyman, B.T. (2000b). Apolipoprotein E facilitates neuritic and cerebrovascular plaque formation in an Alzheimer's disease model. Annals of neurology 47, 739747.

Hong, S., Beja-Glasser, V.F., Nfonoyim, B.M., Frouin, A., Li, S., Ramakrishnan, S., Merry, K.M., Shi, Q., Rosenthal, A., Barres, B.A., et al. (2016). Complement and microglia mediate early synapse loss in Alzheimer mouse models. Science 352, 712-716.

Hornung, V., Bauernfeind, F., Halle, A., Samstad, E.O., Kono, H., Rock, K.L., Fitzgerald, K.A., and Latz, E. (2008). Silica crystals and aluminum salts activate the NALP3 inflammasome through phagosomal destabilization. Nature immunology 9, 847.

Hoshino, T., Suzuki, K., Matsushima, T., Yamakawa, N., Suzuki, T., and Mizushima, T. (2013). Suppression of Alzheimer's disease-related phenotypes by geranylgeranylacetone in mice. PloS one 8 , e76306.

Hsiao, K., Chapman, P., Nilsen, S., Eckman, C., Harigaya, Y., Younkin, S., Yang, F., and Cole, G. (1996). Correlative memory deficits, Abeta elevation, and amyloid plaques in transgenic mice. Science 274, 99-102.

Huang, Y., and Mucke, L. (2012). Alzheimer mechanisms and therapeutic strategies. Cell 148, 1204-1222.

Huang, Y.A., Zhou, B., Wernig, M., and Sudhof, T.C. (2017). ApoE2, ApoE3, and ApoE4 Differentially Stimulate APP Transcription and Abeta Secretion. Cell 168, 427-441.e421.

Hughes, P.M., Botham, M.S., Frentzel, S., Mir, A., and Perry, V.H. (2002). Expression of fractalkine (CX3CL1) and its receptor, CX3CR1, during acute and chronic inflammation in the rodent CNS. Glia 37, 314-327.

Hutagalung, A.H., and Novick, P.J. (2011). Role of Rab GTPases in membrane traffic and cell physiology. Physiological reviews 91, 119-149.

Hyttinen, J.M.T., Niittykoski, M., Salminen, A., and Kaarniranta, K. (2013). Maturation of autophagosomes and endosomes: A key role for Rab7. Biochimica et Biophysica Acta (BBA) Molecular Cell Research 1833, 503-510.

Imai, T., Hieshima, K., Haskell, C., Baba, M., Nagira, M., Nishimura, M., Kakizaki, M., Takagi, S., Nomiyama, H., Schall, T.J., et al. (1997). Identification and molecular characterization of fractalkine receptor CX3CR1, which mediates both leukocyte migration and adhesion. Cell 91, 521-530. 
Imai, Y., Ibata, I., Ito, D., Ohsawa, K., and Kohsaka, S. (1996). A novel gene iba1 in the major histocompatibility complex class III region encoding an EF hand protein expressed in a monocytic lineage. Biochemical and biophysical research communications 224, 855-862.

Itagaki, S., McGeer, P.L., and Akiyama, H. (1988). Presence of T-cytotoxic suppressor and leucocyte common antigen positive cells in Alzheimer's disease brain tissue. Neuroscience letters 91, 259-264.

Ito, D., Imai, Y., Ohsawa, K., Nakajima, K., Fukuuchi, Y., and Kohsaka, S. (1998). Microglia-specific localisation of a novel calcium binding protein, Iba1. Brain research Molecular brain research 57, 19.

Iwata, N., Sekiguchi, M., Hattori, Y., Takahashi, A., Asai, M., Ji, B., Higuchi, M., Staufenbiel, M., Muramatsu, S., and Saido, T.C. (2013). Global brain delivery of neprilysin gene by intravascular administration of AAV vector in mice. Scientific reports 3, 1472.

Iwata, N., Tsubuki, S., Takaki, Y., Shirotani, K., Lu, B., Gerard, N.P., Gerard, C., Hama, E., Lee, H.J., and Saido, T.C. (2001). Metabolic regulation of brain Abeta by neprilysin. Science 292, 15501552.

Jacobsen, J.S., Wu, C.C., Redwine, J.M., Comery, T.A., Arias, R., Bowlby, M., Martone, R., Morrison, J.H., Pangalos, M.N., Reinhart, P.H., et al. (2006). Early-onset behavioral and synaptic deficits in a mouse model of Alzheimer's disease. Proceedings of the National Academy of Sciences of the United States of America 103, 5161-5166.

Jäger, S., Bucci, C., Tanida, I., Ueno, T., Kominami, E., Saftig, P., and Eskelinen, E.-L. (2004). Role for Rab7 in maturation of late autophagic vacuoles. Journal of cell science $117,4837-4848$.

Jager, S., Bucci, C., Tanida, I., Ueno, T., Kominami, E., Saftig, P., and Eskelinen, E.L. (2004). Role for Rab7 in maturation of late autophagic vacuoles. Journal of cell science 117, 4837-4848.

Jahn, H. (2013). Memory loss in Alzheimer's disease. Dialogues in clinical neuroscience 15, 445454.

Jawhar, S., Trawicka, A., Jenneckens, C., Bayer, T.A., and Wirths, O. (2012). Motor deficits, neuron loss, and reduced anxiety coinciding with axonal degeneration and intraneuronal $A \beta$ aggregation in the 5XFAD mouse model of Alzheimer's disease. Neurobiology of aging 33, 196.e129-196.e140.

Jay, T.R., Hirsch, A.M., Broihier, M.L., Miller, C.M., Neilson, L.E., Ransohoff, R.M., Lamb, B.T., and Landreth, G.E. (2017). Disease progression-dependent effects of TREM2 deficiency in a mouse model of Alzheimer's disease. J Neurosci 37.

Jay, T.R., Miller, C.M., Cheng, P.J., Graham, L.C., Bemiller, S., Broihier, M.L., Xu, G., Margevicius, D., Karlo, J.C., Sousa, G.L., et al. (2015). TREM2 deficiency eliminates TREM2+ inflammatory macrophages and ameliorates pathology in Alzheimer's disease mouse models. The Journal of experimental medicine 212.

Jefferson, T., Causevic, M., auf dem Keller, U., Schilling, O., Isbert, S., Geyer, R., Maier, W., Tschickardt, S., Jumpertz, T., Weggen, S., et al. (2011). Metalloprotease meprin beta generates nontoxic $\mathrm{N}$-terminal amyloid precursor protein fragments in vivo. The Journal of biological chemistry 286, 27741-27750.

Jiang, H., Burdick, D., Glabe, C.G., Cotman, C.W., and Tenner, A.J. (1994). beta-Amyloid activates complement by binding to a specific region of the collagen-like domain of the $\mathrm{C} 1 \mathrm{q} A$ chain. Journal of immunology (Baltimore, Md : 1950) 152, 5050-5059. 
Johnson, S.C., Christian, B.T., Okonkwo, O.C., Oh, J.M., Harding, S., Xu, G., Hillmer, A.T., Wooten, D.W., Murali, D., Barnhart, T.E., et al. (2014). Amyloid burden and neural function in people at risk for Alzheimer's Disease. Neurobiology of aging 35, 576-584.

Jones, R.S., Minogue, A.M., Connor, T.J., and Lynch, M.A. (2013). Amyloid-beta-induced astrocytic phagocytosis is mediated by CD36, CD47 and RAGE. Journal of neuroimmune pharmacology : the official journal of the Society on Neurolmmune Pharmacology 8, 301-311.

Jung, D.Y., Lee, H., Jung, B.Y., Ock, J., Lee, M.S., Lee, W.H., and Suk, K. (2005). TLR4, but not TLR2, signals autoregulatory apoptosis of cultured microglia: a critical role of IFN-beta as a decision maker. Journal of immunology (Baltimore, Md : 1950) 174, 6467-6476.

Kabeya, Y., Mizushima, N., Ueno, T., Yamamoto, A., Kirisako, T., Noda, T., Kominami, E., Ohsumi, Y., and Yoshimori, T. (2000). LC3, a mammalian homologue of yeast Apg8p, is localized in autophagosome membranes after processing. The EMBO journal 19, 5720-5728.

Kamphuis, W., Kooijman, L., Orre, M., Stassen, O., Pekny, M., and Hol, E.M. (2015). GFAP and vimentin deficiency alters gene expression in astrocytes and microglia in wild-type mice and changes the transcriptional response of reactive glia in mouse model for Alzheimer's disease. Glia $63,1036-1056$.

Kang, J.H., and Grodstein, F. (2012). Postmenopausal hormone therapy, timing of initiation, APOE and cognitive decline. Neurobiology of aging 33,1129-1137.

Kang, S.S., Ebbert, M.T.W., Baker, K.E., Cook, C., Wang, X., Sens, J.P., Kocher, J.-P., Petrucelli, L., and Fryer, J.D. (2018). Microglial translational profiling reveals a convergent APOE pathway from aging, amyloid, and tau. The Journal of experimental medicine 215, 2235-2245.

Karran, E., Mercken, M., and Strooper, B.D. (2011). The amyloid cascade hypothesis for Alzheimer\&\#39;s disease: an appraisal for the development of therapeutics. Nature Reviews Drug Discovery $10,698$.

Kaur, J., and Debnath, J. (2015). Autophagy at the crossroads of catabolism and anabolism. Nature Reviews Molecular Cell Biology 16, 461.

Keaney, J., Walsh, D.M., O'Malley, T., Hudson, N., Crosbie, D.E., Loftus, T., Sheehan, F., McDaid, J., Humphries, M.M., Callanan, J.J., et al. (2015). Autoregulated paracellular clearance of amyloid- $\beta$ across the blood-brain barrier. Science Advances 1.

Keren-Shaul, H., Spinrad, A., Weiner, A., Matcovitch-Natan, O., Dvir-Szternfeld, R., Ulland, T.K., David, E., Baruch, K., Lara-Astaiso, D., Toth, B., et al. (2017). A Unique Microglia Type Associated with Restricting Development of Alzheimer's Disease. Cell 169, 1276-1290.e1217.

Kessaris, N., Pringle, N., and Richardson, W.D. (2008). Specification of CNS glia from neural stem cells in the embryonic neuroepithelium. Philosophical transactions of the Royal Society of London Series B, Biological sciences 363, 71-85.

Kettenmann, H., Hanisch, U.K., Noda, M., and Verkhratsky, A. (2011). Physiology of microglia. Physiological reviews 91, 461-553.

Kidd, M. (1963). Paired helical filaments in electron microscopy of Alzheimer's disease. Nature 197, 192-193.

Kierdorf, K., Erny, D., Goldmann, T., Sander, V., Schulz, C., Perdiguero, E.G., Wieghofer, P., Heinrich, A., Riemke, P., Hölscher, C., et al. (2013). Microglia emerge from erythromyeloid precursors via Pu.1- and Irf8-dependent pathways. Nature neuroscience 16, 273. 
Kim, H.J., Cho, M.H., Shim, W.H., Kim, J.K., Jeon, E.Y., Kim, D.H., and Yoon, S.Y. (2017). Deficient autophagy in microglia impairs synaptic pruning and causes social behavioral defects. Molecular psychiatry $22,1576-1584$.

Kim, W.K., Alvarez, X., Fisher, J., Bronfin, B., Westmoreland, S., McLaurin, J., and Williams, K. (2006). CD163 identifies perivascular macrophages in normal and viral encephalitic brains and potential precursors to perivascular macrophages in blood. The American journal of pathology 168 , 822-834.

Kimura, R., and Ohno, M. (2009). Impairments in remote memory stabilization precede hippocampal synaptic and cognitive failures in 5XFAD Alzheimer mouse model. Neurobiology of disease 33, 229-235.

Kiral, F.R., Kohrs, F.E., Jin, E.J., and Hiesinger, P.R. (2018). Rab GTPases and Membrane Trafficking in Neurodegeneration. Current biology : CB 28, R471-R486.

Kleinberger, G., Yamanishi, Y., Suarez-Calvet, M., Czirr, E., Lohmann, E., Cuyvers, E., Struyfs, H., Pettkus, N., Wenninger-Weinzierl, A., Mazaheri, F., et al. (2014). TREM2 mutations implicated in neurodegeneration impair cell surface transport and phagocytosis. Science translational medicine 6,243 ra286.

Klunk, W.E., Bacskai, B.J., Mathis, C.A., Kajdasz, S.T., McLellan, M.E., Frosch, M.P., Debnath, M.L., Holt, D.P., Wang, Y., and Hyman, B.T. (2002). Imaging Abeta plaques in living transgenic mice with multiphoton microscopy and methoxy-X04, a systemically administered Congo red derivative. Journal of neuropathology and experimental neurology 61, 797-805.

Knobloch, M., and Mansuy, I.M. (2008). Dendritic Spine Loss and Synaptic Alterations in Alzheimer's Disease. Molecular Neurobiology 37, 73-82.

Komatsu, M., Waguri, S., Chiba, T., Murata, S., Iwata, J., Tanida, I., Ueno, T., Koike, M., Uchiyama, Y., Kominami, E., et al. (2006). Loss of autophagy in the central nervous system causes neurodegeneration in mice. Nature 441, 880-884.

Kopke, E., Tung, Y.C., Shaikh, S., Alonso, A.C., lqbal, K., and Grundke-lqbal, I. (1993). Microtubule-associated protein tau. Abnormal phosphorylation of a non-paired helical filament pool in Alzheimer disease. The Journal of biological chemistry 268, 24374-24384.

Kraft, A.W., Hu, X., Yoon, H., Yan, P., Xiao, Q., Wang, Y., Gil, S.C., Brown, J., Wilhelmsson, U., Restivo, J.L., et al. (2012). Attenuating astrocyte activation accelerates plaque pathogenesis in APP/PS1 mice. The FASEB Journal 27, 187-198.

Krasemann, S., Madore, C., Cialic, R., Baufeld, C., Calcagno, N., El Fatimy, R., Beckers, L., O'Loughlin, E., Xu, Y., Fanek, Z., et al. (2017). The TREM2-APOE Pathway Drives the Transcriptional Phenotype of Dysfunctional Microglia in Neurodegenerative Diseases. Immunity 47, 566-581.e569.

Kuchitsu, Y., Homma, Y., Fujita, N., and Fukuda, M. (2018). Rab7 knockout unveils regulated autolysosome maturation induced by glutamine starvation. Journal of cell science 131 .

Kummer, M.P., and Heneka, M.T. (2014). Truncated and modified amyloid-beta species. Alzheimer's research \& therapy 6, 28-28.

Kunkle, B.W., Vardarajan, B.N., Naj, A.C., Whitehead, P.L., Rolati, S., Slifer, S., Carney, R.M., Cuccaro, M.L., Vance, J.M., Gilbert, J.R., et al. (2017). Early-Onset Alzheimer Disease and Candidate Risk Genes Involved in Endolysosomal Transport. JAMA Neurol 74, 1113-1122. 
LaFerla, F.M., Troncoso, J.C., Strickland, D.K., Kawas, C.H., and Jay, G. (1997). Neuronal cell death in Alzheimer's disease correlates with apoE uptake and intracellular Abeta stabilization. The Journal of clinical investigation 100, 310-320.

Lai, A.Y., Dibal, C.D., Armitage, G.A., Winship, I.R., and Todd, K.G. (2013). Distinct activation profiles in microglia of different ages: a systematic study in isolated embryonic to aged microglial cultures. Neuroscience 254, 185-195.

Lam, B., Masellis, M., Freedman, M., Stuss, D.T., and Black, S.E. (2013). Clinical, imaging, and pathological heterogeneity of the Alzheimer's disease syndrome. Alzheimer's research \& therapy 5 , 1.

Lambert, J.C., Heath, S., Even, G., Campion, D., Sleegers, K., Hiltunen, M., Combarros, O., Zelenika, D., Bullido, M.J., Tavernier, B., et al. (2009). Genome-wide association study identifies variants at CLU and CR1 associated with Alzheimer's disease. Nature genetics 41, 1094-1099.

Lambert, J.C., Ibrahim-Verbaas, C.A., Harold, D., Naj, A.C., Sims, R., Bellenguez, C., DeStafano, A.L., Bis, J.C., Beecham, G.W., Grenier-Boley, B., et al. (2013). Meta-analysis of 74,046 individuals identifies 11 new susceptibility loci for Alzheimer's disease. Nature genetics 45, 1452-1458.

Lambert, M.P., Barlow, A.K., Chromy, B.A., Edwards, C., Freed, R., Liosatos, M., Morgan, T.E., Rozovsky, I., Trommer, B., Viola, K.L., et al. (1998). Diffusible, nonfibrillar ligands derived from Abeta1-42 are potent central nervous system neurotoxins. Proceedings of the National Academy of Sciences of the United States of America 95, 6448-6453.

Landel, V., Baranger, K., Virard, I., Loriod, B., Khrestchatisky, M., Rivera, S., Benech, P., and Feron, F. (2014). Temporal gene profiling of the 5XFAD transgenic mouse model highlights the importance of microglial activation in Alzheimer's disease. Molecular neurodegeneration 9, 33.

Lapenna, A., De Palma, M., and Lewis, C.E. (2018). Perivascular macrophages in health and disease. Nature Reviews Immunology.

Lee, C.Y., Tse, W., Smith, J.D., and Landreth, G.E. (2012). Apolipoprotein E promotes beta-amyloid trafficking and degradation by modulating microglial cholesterol levels. The Journal of biological chemistry $287,2032-2044$.

Lee, C.Y.D., Daggett, A., Gu, X., Jiang, L.L., Langfelder, P., Li, X., Wang, N., Zhao, Y., Park, C.S., Cooper, Y., et al. (2018). Elevated TREM2 Gene Dosage Reprograms Microglia Responsivity and Ameliorates Pathological Phenotypes in Alzheimer's Disease Models. Neuron 97, 10321048.e1035.

Lee, J.-H., Yu, W.H., Kumar, A., Lee, S., Mohan, P.S., Peterhoff, C.M., Wolfe, D.M., MartinezVicente, M., Massey, A.C., Sovak, G., et al. (2010). Lysosomal Proteolysis and Autophagy Require Presenilin 1 and Are Disrupted by Alzheimer-Related PS1 Mutations. Cell 141, 1146-1158.

Lee, S., Sato, Y., and Nixon, R.A. (2011). Lysosomal proteolysis inhibition selectively disrupts axonal transport of degradative organelles and causes an Alzheimer's-like axonal dystrophy. $\mathrm{J}$ Neurosci 31, 7817-7830.

Lemere, C.A., Blusztajn, J.K., Yamaguchi, H., Wisniewski, T., Saido, T.C., and Selkoe, D.J. (1996). Sequence of deposition of heterogeneous amyloid beta-peptides and APO E in Down syndrome: implications for initial events in amyloid plaque formation. Neurobiology of disease 3, 16-32.

Lenz, K.M., and Nelson, L.H. (2018). Microglia and Beyond: Innate Immune Cells As Regulators of Brain Development and Behavioral Function. Frontiers in immunology 9, 698. 
Lenz, K.M., Nugent, B.M., Haliyur, R., and McCarthy, M.M. (2013). Microglia are essential to masculinization of brain and behavior. J Neurosci 33, 2761-2772.

Letiembre, M., Hao, W., Liu, Y., Walter, S., Mihaljevic, I., Rivest, S., Hartmann, T., and Fassbender, K. (2007). Innate immune receptor expression in normal brain aging. Neuroscience 146, 248-254.

Leung, K.K., Bartlett, J.W., Barnes, J., Manning, E.N., Ourselin, S., Fox, N.C., and for the Alzheimer's Disease Neuroimaging, I. (2013). Cerebral atrophy in mild cognitive impairment and Alzheimer disease: Rates and acceleration. Neurology 80,648-654.

Levy-Lahad, E., Wasco, W., Poorkaj, P., Romano, D.M., Oshima, J., Pettingell, W.H., Yu, C.E., Jondro, P.D., Schmidt, S.D., Wang, K., et al. (1995). Candidate gene for the chromosome 1 familial Alzheimer's disease locus. Science 269, 973-977.

Levy, E., Carman, M.D., Fernandez-Madrid, I.J., Power, M.D., Lieberburg, I., van Duinen, S.G., Bots, G.T., Luyendijk, W., and Frangione, B. (1990). Mutation of the Alzheimer's disease amyloid gene in hereditary cerebral hemorrhage, Dutch type. Science 248, 1124-1126.

Liddelow, S.A., Guttenplan, K.A., Clarke, L.E., Bennett, F.C., Bohlen, C.J., Schirmer, L., Bennett, M.L., Münch, A.E., Chung, W.-S., Peterson, T.C., et al. (2017). Neurotoxic reactive astrocytes are induced by activated microglia. Nature $541,481$.

Lin, Y.T., Seo, J., Gao, F., Feldman, H.M., Wen, H.L., Penney, J., Cam, H.P., Gjoneska, E., Raja, W.K., Cheng, J., et al. (2018). APOE4 Causes Widespread Molecular and Cellular Alterations Associated with Alzheimer's Disease Phenotypes in Human iPSC-Derived Brain Cell Types. Neuron 98, 1141-1154.e1147.

Lindsay, J., Laurin, D., Verreault, R., Hebert, R., Helliwell, B., Hill, G.B., and McDowell, I. (2002). Risk factors for Alzheimer's disease: a prospective analysis from the Canadian Study of Health and Aging. American journal of epidemiology 156, 445-453.

Linebaugh, B.E., Sameni, M., Day, N.A., Sloane, B.F., and Keppler, D. (1999). Exocytosis of active cathepsin B. European Journal of Biochemistry 264, 100-109.

Livingston, G., Sommerlad, A., Orgeta, V., Costafreda, S.G., Huntley, J., Ames, D., Ballard, C., Banerjee, S., Burns, A., Cohen-Mansfield, J., et al. (2017). Dementia prevention, intervention, and care. Lancet (London, England) 390, 2673-2734.

Lööv, C., Mitchell, C.H., Simonsson, M., and Erlandsson, A. (2015). Slow degradation in phagocytic astrocytes can be enhanced by lysosomal acidification. Glia 63, 1997-2009.

Lue, L.F., Kuo, Y.M., Roher, A.E., Brachova, L., Shen, Y., Sue, L., Beach, T., Kurth, J.H., Rydel, R.E., and Rogers, J. (1999). Soluble amyloid beta peptide concentration as a predictor of synaptic change in Alzheimer's disease. The American journal of pathology 155, 853-862.

Luebke, J.I., Weaver, C.M., Rocher, A.B., Rodriguez, A., Crimins, J.L., Dickstein, D.L., Wearne, S.L., and Hof, P.R. (2010). Dendritic vulnerability in neurodegenerative disease: insights from analyses of cortical pyramidal neurons in transgenic mouse models. Brain Structure and Function 214, 181-199.

Lui, H., Zhang, J., Makinson, S.R., Cahill, M.K., Kelley, K.W., Huang, H.-Y., Shang, Y., Oldham, M.C., Martens, L.H., Gao, F., et al. (2016). Progranulin Deficiency Promotes Circuit-Specific Synaptic Pruning by Microglia via Complement Activation. Cell 165, 921-935.

Luzio, J.P., Pryor, P.R., and Bright, N.A. (2007). Lysosomes: fusion and function. Nature Reviews Molecular Cell Biology 8, 622. 
Lynch, A.M., Murphy, K.J., Deighan, B.F., O'Reilly, J.-A., Gun'ko, Y.K., Cowley, T.R., GonzalezReyes, R.E., and Lynch, M.A. (2010). The Impact of Glial Activation in the Aging Brain. Aging and Disease 1, 262-278.

Ma, Q.-L., Yang, F., Rosario, E.R., Ubeda, O.J., Beech, W., Gant, D.J., Chen, P.P., Hudspeth, B., Chen, C., Zhao, Y., et al. (2009). $\beta$-Amyloid Oligomers Induce Phosphorylation of Tau and Inactivation of Insulin Receptor Substrate via c-Jun N-Terminal Kinase Signaling: Suppression by Omega-3 Fatty Acids and Curcumin. The Journal of Neuroscience 29, 9078-9089.

Mahley, R.W., and Rall, S.C., Jr. (2000). Apolipoprotein E: far more than a lipid transport protein. Annual review of genomics and human genetics 1, 507-537.

Mahley, R.W., Weisgraber, K.H., and Huang, Y. (2006). Apolipoprotein E4: a causative factor and therapeutic target in neuropathology, including Alzheimer's disease. Proceedings of the National Academy of Sciences of the United States of America 103, 5644-5651.

Maier, M., Peng, Y., Jiang, L., Seabrook, T.J., Carroll, M.C., and Lemere, C.A. (2008). Complement C3 deficiency leads to accelerated amyloid beta plaque deposition and neurodegeneration and modulation of the microglia/macrophage phenotype in amyloid precursor protein transgenic mice. $J$ Neurosci 28, 6333-6341.

Malm, T.M., Koistinaho, M., Parepalo, M., Vatanen, T., Ooka, A., Karlsson, S., and Koistinaho, J. (2005). Bone-marrow-derived cells contribute to the recruitment of microglial cells in response to beta-amyloid deposition in APP/PS1 double transgenic Alzheimer mice. Neurobiology of disease 18, 134-142.

Martens, L.H., Zhang, J., Barmada, S.J., Zhou, P., Kamiya, S., Sun, B., Min, S.W., Gan, L., Finkbeiner, S., Huang, E.J., et al. (2012). Progranulin deficiency promotes neuroinflammation and neuron loss following toxin-induced injury. The Journal of clinical investigation 122, 3955-3959.

Martin, E., Boucher, C., Fontaine, B., and Delarasse, C. (2017). Distinct inflammatory phenotypes of microglia and monocyte-derived macrophages in Alzheimer's disease models: effects of aging and amyloid pathology. Aging cell 16, 27-38.

Masliah, E., Sisk, A., Mallory, M., Mucke, L., Schenk, D., and Games, D. (1996). Comparison of neurodegenerative pathology in transgenic mice overexpressing V717F beta-amyloid precursor protein and Alzheimer's disease. J Neurosci 16, 5795-5811.

Mass, E., Ballesteros, I., Farlik, M., Halbritter, F., Günther, P., Crozet, L., Jacome-Galarza, C.E., Händler, K., Klughammer, J., Kobayashi, Y., et al. (2016). Specification of tissue-resident macrophages during organogenesis. Science.

Mass, E., Jacome-Galarza, C.E., Blank, T., Lazarov, T., Durham, B.H., Ozkaya, N., Pastore, A., Schwabenland, M., Chung, Y.R., Rosenblum, M.K., et al. (2017). A somatic mutation in erythromyeloid progenitors causes neurodegenerative disease. Nature 549, 389-393.

Masters, C.L., Bateman, R., Blennow, K., Rowe, C.C., Sperling, R.A., and Cummings, J.L. (2015). Alzheimer's disease. Nature Reviews Disease Primers 1, 15056.

Masters, C.L., Simms, G., Weinman, N.A., Multhaup, G., McDonald, B.L., and Beyreuther, K. (1985). Amyloid plaque core protein in Alzheimer disease and Down syndrome. Proceedings of the National Academy of Sciences of the United States of America 82, 4245-4249.

Matcovitch-Natan, O., Winter, D.R., Giladi, A., Vargas Aguilar, S., Spinrad, A., Sarrazin, S., BenYehuda, H., David, E., Zelada Gonzalez, F., Perrin, P., et al. (2016). Microglia development follows a stepwise program to regulate brain homeostasis. Science 353 , aad8670. 
Mathys, H., Adaikkan, C., Gao, F., Young, J.Z., Manet, E., Hemberg, M., De Jager, P.L., Ransohoff, R.M., Regev, A., and Tsai, L.-H. (2017). Temporal Tracking of Microglia Activation in Neurodegeneration at Single-Cell Resolution. Cell Reports 21, 366-380.

Mato, M., Ookawara, S., Mato, T.K., and Namiki, T. (1985). An attempt to differentiate further between microglia and fluorescent granular perithelial (FGP) cells by their capacity to incorporate exogenous protein. The American journal of anatomy 172, 125-140.

Mato, M., Ookawara, S., Sakamoto, A., Aikawa, E., Ogawa, T., Mitsuhashi, U., Masuzawa, T., Suzuki, H., Honda, M., Yazaki, Y., et al. (1996). Involvement of specific macrophage-lineage cells surrounding arterioles in barrier and scavenger function in brain cortex. Proceedings of the National Academy of Sciences of the United States of America 93, 3269-3274.

Matsui, T., Ingelsson, M., Fukumoto, H., Ramasamy, K., Kowa, H., Frosch, M.P., Irizarry, M.C., and Hyman, B.T. (2007). Expression of APP pathway mRNAs and proteins in Alzheimer's disease. Brain research 1161, 116-123.

McCray, B.A., Skordalakes, E., and Taylor, J.P. (2010). Disease mutations in Rab7 result in unregulated nucleotide exchange and inappropriate activation. Human molecular genetics 19, 1033-1047.

Medina, M., Khachaturian, Z.S., Rossor, M., Avila, J., and Cedazo-Minguez, A. (2017). Toward common mechanisms for risk factors in Alzheimer's syndrome. Alzheimer's \& Dementia:

Translational Research \& Clinical Interventions 3, 571-578.

Melchor, J.P., Pawlak, R., and Strickland, S. (2003). The tissue plasminogen activator-plasminogen proteolytic cascade accelerates amyloid-beta (Abeta) degradation and inhibits Abeta-induced neurodegeneration. J Neurosci 23, 8867-8871.

Mildner, A., Schmidt, H., Nitsche, M., Merkler, D., Hanisch, U.K., Mack, M., Heikenwalder, M., Bruck, W., Priller, J., and Prinz, M. (2007). Microglia in the adult brain arise from Ly-6ChiCCR2+ monocytes only under defined host conditions. Nature neuroscience 10, 1544-1553.

Minett, T., Classey, J., Matthews, F.E., Fahrenhold, M., Taga, M., Brayne, C., Ince, P.G., Nicoll, J.A., and Boche, D. (2016). Microglial immunophenotype in dementia with Alzheimer's pathology. Journal of neuroinflammation 13, 135.

Mirra, S.S., Heyman, A., McKeel, D., Sumi, S.M., Crain, B.J., Brownlee, L.M., Vogel, F.S., Hughes, J.P., van Belle, G., and Berg, L. (1991). The Consortium to Establish a Registry for Alzheimer's Disease (CERAD). Part II. Standardization of the neuropathologic assessment of Alzheimer's disease. Neurology 41, 479-486.

Mitrasinovic, O.M., Vincent, V.A.M., Simsek, D., and Murphy, G.M. (2003). Macrophage colony stimulating factor promotes phagocytosis by murine microglia. Neuroscience letters 344, 185-188.

Mizutani, M., Pino, P.A., Saederup, N., Charo, I.F., Ransohoff, R.M., and Cardona, A.E. (2012). The fractalkine receptor but not CCR2 is present on microglia from embryonic development throughout adulthood. Journal of immunology (Baltimore, Md : 1950) 188, 29-36.

Moraga, A., Pradillo, J.M., García-Culebras, A., Palma-Tortosa, S., Ballesteros, I., HernándezJiménez, M., Moro, M.A., and Lizasoain, I. (2015). Aging increases microglial proliferation, delays cell migration, and decreases cortical neurogenesis after focal cerebral ischemia. Journal of neuroinflammation $12,87$.

Mosher, K.I., and Wyss-Coray, T. (2014). Microglial Dysfunction in Brain Aging and Alzheimer's Disease. Biochemical pharmacology 88, 594-604. 
Mosser, C.A., Baptista, S., Arnoux, I., and Audinat, E. (2017). Microglia in CNS development: Shaping the brain for the future. Progress in neurobiology 149-150, 1-20.

Mucke, L., Masliah, E., Yu, G.Q., Mallory, M., Rockenstein, E.M., Tatsuno, G., Hu, K., Kholodenko, D., Johnson-Wood, K., and McConlogue, L. (2000). High-level neuronal expression of abeta 1-42 in wild-type human amyloid protein precursor transgenic mice: synaptotoxicity without plaque formation. J Neurosci 20, 4050-4058.

Mueller-Steiner, S., Zhou, Y., Arai, H., Roberson, E.D., Sun, B., Chen, J., Wang, X., Yu, G., Esposito, L., Mucke, L., et al. (2006). Antiamyloidogenic and Neuroprotective Functions of Cathepsin B: Implications for Alzheimer's Disease. Neuron 51, 703-714.

Mullan, M., Crawford, F., Axelman, K., Houlden, H., Lilius, L., Winblad, B., and Lannfelt, L. (1992). A pathogenic mutation for probable Alzheimer's disease in the APP gene at the N-terminus of betaamyloid. Nature genetics 1, 345-347.

Murrell, J., Farlow, M., Ghetti, B., and Benson, M.D. (1991). A mutation in the amyloid precursor protein associated with hereditary Alzheimer's disease. Science 254, 97-99.

Naert, G., and Rivest, S. (2011). CC chemokine receptor 2 deficiency aggravates cognitive impairments and amyloid pathology in a transgenic mouse model of Alzheimer's disease. $J$ Neurosci 31, 6208-6220.

Nagele, R.G., D'Andrea, M.R., Lee, H., Venkataraman, V., and Wang, H.Y. (2003). Astrocytes accumulate A beta 42 and give rise to astrocytic amyloid plaques in Alzheimer disease brains. Brain research 971, 197-209.

Nakamura, S., and Yoshimori, T. (2017). New insights into autophagosome-lysosome fusion. Journal of cell science 130, 1209-1216.

Nam, K.N., Mounier, A., Wolfe, C.M., Fitz, N.F., Carter, A.Y., Castranio, E.L., Kamboh, H.I., Reeves, V.L., Wang, J., Han, X., et al. (2017). Effect of high fat diet on phenotype, brain transcriptome and lipidome in Alzheimer's model mice. Scientific reports 7, 4307.

Namba, Y., Tomonaga, M., Kawasaki, H., Otomo, E., and Ikeda, K. (1991). Apolipoprotein E immunoreactivity in cerebral amyloid deposits and neurofibrillary tangles in Alzheimer's disease and kuru plaque amyloid in Creutzfeldt-Jakob disease. Brain research 541, 163-166.

Narayan, P., Orte, A., Clarke, R.W., Bolognesi, B., Hook, S., Ganzinger, K.A., Meehan, S., Wilson, M.R., Dobson, C.M., and Klenerman, D. (2011). The extracellular chaperone clusterin sequesters oligomeric forms of the amyloid- $\beta 1-40$ peptide. Nature Structural \&Amp; Molecular Biology 19, 79.

Navarro, V., Sanchez-Mejias, E., Jimenez, S., Muñoz-Castro, C., Sanchez-Varo, R., Davila, J.C., Vizuete, M., Gutierrez, A., and Vitorica, J. (2018). Microglia in Alzheimer's Disease: Activated, Dysfunctional or Degenerative. Frontiers in Aging Neuroscience 10, 140.

Neu, S.C., Pa, J., Kukull, W., and et al. (2017). Apolipoprotein e genotype and sex risk factors for alzheimer disease: A meta-analysis. JAMA Neurology 74, 1178-1189.

Nimmerjahn, A., Kirchhoff, F., and Helmchen, F. (2005). Resting microglial cells are highly dynamic surveillants of brain parenchyma in vivo. Science 308, 1314-1318.

Nixon, R.A., Wegiel, J., Kumar, A., Yu, W.H., Peterhoff, C., Cataldo, A., and Cuervo, A.M. (2005). Extensive involvement of autophagy in Alzheimer disease: an immuno-electron microscopy study. Journal of neuropathology and experimental neurology 64, 113-122. 
Njie, E.G., Boelen, E., Stassen, F.R., Steinbusch, H.W., Borchelt, D.R., and Streit, W.J. (2012). Ex vivo cultures of microglia from young and aged rodent brain reveal age-related changes in microglial function. Neurobiology of aging 33, 195.e191-112.

Nuriel, T., Peng, K.Y., Ashok, A., Dillman, A.A., Figueroa, H.Y., Apuzzo, J., Ambat, J., Levy, E., Cookson, M.R., Mathews, P.M., et al. (2017). The Endosomal-Lysosomal Pathway Is Dysregulated by APOE4 Expression in Vivo. Frontiers in neuroscience 11, 702.

Nussbaum, J.M., Schilling, S., Cynis, H., Silva, A., Swanson, E., Wangsanut, T., Tayler, K., Wiltgen, B., Hatami, A., Rönicke, R., et al. (2012). Prion-like behaviour and tau-dependent cytotoxicity of pyroglutamylated amyloid- $\beta$. Nature 485,651 .

O'Leary, T., Robertson, A., Chipman, P., Rafuse, V., and Brown, R. (2013). Motor dysfunction in the 12-month-old 5xFAD mouse model of Alzheimer's disease. Alzheimer's \& Dementia: The Journal of the Alzheimer's Association 9, P497.

Oakley, H., Cole, S.L., Logan, S., Maus, E., Shao, P., Craft, J., Guillozet-Bongaarts, A., Ohno, M., Disterhoft, J., Van Eldik, L., et al. (2006). Intraneuronal beta-amyloid aggregates, neurodegeneration, and neuron loss in transgenic mice with five familial Alzheimer's disease mutations: potential factors in amyloid plaque formation. J Neurosci 26, 10129-10140.

Oddo, S., Billings, L., Kesslak, J.P., Cribbs, D.H., and LaFerla, F.M. (2004). Abeta immunotherapy leads to clearance of early, but not late, hyperphosphorylated tau aggregates via the proteasome. Neuron 43, 321-332.

Oddo, S., Caccamo, A., Kitazawa, M., Tseng, B.P., and LaFerla, F.M. (2003). Amyloid deposition precedes tangle formation in a triple transgenic model of Alzheimer's disease. Neurobiology of aging 24, 1063-1070.

Ofengeim, D., Mazzitelli, S., Ito, Y., DeWitt, J.P., Mifflin, L., Zou, C., Das, S., Adiconis, X., Chen, H., Zhu, H., et al. (2017). RIPK1 mediates a disease-associated microglial response in Alzheimer's disease. Proceedings of the National Academy of Sciences of the United States of America 114, E8788-e8797.

Ogura, K., Ogawa, M., and Yoshida, M. (1994). Effects of ageing on microglia in the normal rat brain: immunohistochemical observations. Neuroreport 5, 1224-1226.

Olmos-Alonso, A., Schetters, S.T., Sri, S., Askew, K., Mancuso, R., Vargas-Caballero, M., Holscher, C., Perry, V.H., and Gomez-Nicola, D. (2016). Pharmacological targeting of CSF1R inhibits microglial proliferation and prevents the progression of Alzheimer's-like pathology. Brain : a journal of neurology 139, 891-907.

Osman, A.M., Rodhe, J., Shen, X., Dominguez, C.A., Joseph, B., and Blomgren, K. (2017). The Secretome of Microglia Regulate Neural Stem Cell Function. Neuroscience.

Owens, R., Grabert, K., Davies, C.L., Alfieri, A., Antel, J.P., Healy, L.M., and McColl, B.W. (2017). Divergent Neuroinflammatory Regulation of Microglial TREM Expression and Involvement of NFkappaB. Frontiers in cellular neuroscience 11, 56.

Pan, Y., Lloyd, C., Zhou, H., Dolich, S., Deeds, J., Gonzalo, J.A., Vath, J., Gosselin, M., Ma, J., Dussault, B., et al. (1997). Neurotactin, a membrane-anchored chemokine upregulated in brain inflammation. Nature 387, 611-617.

Paolicelli, R.C., Bolasco, G., Pagani, F., Maggi, L., Scianni, M., Panzanelli, P., Giustetto, M., Ferreira, T.A., Guiducci, E., Dumas, L., et al. (2011). Synaptic Pruning by Microglia Is Necessary for Normal Brain Development. Science 333, 1456-1458. 
Parajuli, B., Sonobe, Y., Horiuchi, H., Takeuchi, H., Mizuno, T., and Suzumura, A. (2013). Oligomeric amyloid $\beta$ induces IL-1 $\beta$ processing via production of ROS: implication in Alzheimer's disease. Cell Death \&Amp; Disease 4, e975.

Paz-y-Miño, C.A., García-Cárdenas, J.M., López-Cortés, A., Salazar, C., Leone, P.E., and Serrano, M. (2015). Positive Association of the Cathepsin D Ala224Val Gene Polymorphism With the Risk of Alzheimer's Disease. The American Journal of the Medical Sciences 350, $296-301$.

Peters, A., Josephson, K., and Vincent, S.L. (1991). Effects of aging on the neuroglial cells and pericytes within area 17 of the rhesus monkey cerebral cortex. The Anatomical record 229, 384398.

Pfeiffer, T., Poll, S., Bancelin, S., Angibaud, J., Inavalli, V.V.G.K., Keppler, K., Mittag, M., Fuhrmann, M., and Nägerl, U.V. (2018). Chronic 2P-STED imaging reveals high turnover of dendritic spines in the hippocampus in vivo. eLife 7, e34700.

Plescher, M., Seifert, G., Hansen, J.N., Bedner, P., Steinhauser, C., and Halle, A. (2018). Plaquedependent morphological and electrophysiological heterogeneity of microglia in an Alzheimer's disease mouse model. Glia 66, 1464-1480.

Pottier, C., Ravenscroft, T.A., Brown, P.H., Finch, N.A., Baker, M., Parsons, M., Asmann, Y.W., Ren, Y., Christopher, E., Levitch, D., et al. (2016). TYROBP genetic variants in early-onset Alzheimer's disease. Neurobiology of aging 48, 222.e229-222.e215.

Prinz, M., Priller, J., Sisodia, S.S., and Ransohoff, R.M. (2011). Heterogeneity of CNS myeloid cells and their roles in neurodegeneration. Nature neuroscience 14, 1227.

Radde, R., Bolmont, T., Kaeser, S.A., Coomaraswamy, J., Lindau, D., Stoltze, L., Calhoun, M.E., Jaggi, F., Wolburg, H., Gengler, S., et al. (2006). Abeta42-driven cerebral amyloidosis in transgenic mice reveals early and robust pathology. EMBO reports 7, 940-946.

Rahimian, R., Cordeau, P., and Kriz, J. (2018). Brain Response to Injuries: When Microglia Go Sexist. Neuroscience.

Ramon y Cajal Agüeras, S. (2016). Pío del Río-Hortega: A Visionary in the Pathology of Central Nervous System Tumors. Frontiers in Neuroanatomy 10.

Rangaraju, S., Dammer, E.B., Raza, S.A., Rathakrishnan, P., Xiao, H., Gao, T., Duong, D.M., Pennington, M.W., Lah, J.J., Seyfried, N.T., et al. (2018). Identification and therapeutic modulation of a pro-inflammatory subset of disease-associated-microglia in Alzheimer's disease. Molecular neurodegeneration 13, 24.

Rascovsky, K., Hodges, J.R., Knopman, D., Mendez, M.F., Kramer, J.H., Neuhaus, J., van Swieten, J.C., Seelaar, H., Dopper, E.G.P., Onyike, C.U., et al. (2011). Sensitivity of revised diagnostic criteria for the behavioural variant of frontotemporal dementia. Brain : a journal of neurology 134 , 2456-2477.

Rebeck, G.W., Harr, S.D., Strickland, D.K., and Hyman, B.T. (1995). Multiple, diverse senile plaqueassociated proteins are ligands of an apolipoprotein $E$ receptor, the alpha 2-macroglobulin receptor/low-density-lipoprotein receptor-related protein. Annals of neurology 37, 211-217.

Rebeck, G.W., Reiter, J.S., Strickland, D.K., and Hyman, B.T. (1993). Apolipoprotein E in sporadic Alzheimer's disease: allelic variation and receptor interactions. Neuron 11, 575-580.

Reichenbach, N., Delekate, A., Breithausen, B., Keppler, K., Poll, S., Schulte, T., Peter, J., Plescher, M., Hansen, J.N., Blank, N., et al. (2018). P2Y1 receptor blockade normalizes network 
dysfunction and cognition in an Alzheimer's disease model. The Journal of experimental medicine $215,1649-1663$.

Reichwald, J., Danner, S., Wiederhold, K.H., and Staufenbiel, M. (2009). Expression of complement system components during aging and amyloid deposition in APP transgenic mice. Journal of neuroinflammation 6,35 .

Reiserer, R.S., Harrison, F.E., Syverud, D.C., and McDonald, M.P. (2007). Impaired spatial learning in the APPSwe + PSEN1DeltaE9 bigenic mouse model of Alzheimer's disease. Genes, brain, and behavior 6, 54-65.

Reitz, C., and Mayeux, R. (2014). Alzheimer disease: Epidemiology, Diagnostic Criteria, Risk Factors and Biomarkers. Biochemical pharmacology 88, 640-651.

Replogle, J.M., Chan, G., White, C.C., Raj, T., Winn, P.A., Evans, D.A., Sperling, R.A., Chibnik, L.B., Bradshaw, E.M., Schneider, J.A., et al. (2015). A TREM1 variant alters the accumulation of Alzheimer-related amyloid pathology. Annals of neurology 77, 469-477.

Réu, P., Khosravi, A., Bernard, S., Mold, J.E., Salehpour, M., Alkass, K., Perl, S., Tisdale, J., Possnert, G., Druid, H., et al. (2017). The Lifespan and Turnover of Microglia in the Human Brain. Cell Reports 20, 779-784.

Risher, W.C., Ustunkaya, T., Singh Alvarado, J., and Eroglu, C. (2014). Rapid Golgi Analysis Method for Efficient and Unbiased Classification of Dendritic Spines. PloS one 9, e107591.

Ritzel, R.M., Patel, A.R., Pan, S., Crapser, J., Hammond, M., Jellison, E., and McCullough, L.D. (2015). Age- and location-related changes in microglial function. Neurobiology of aging 36, 21532163.

Roberson, E.D., Scearce-Levie, K., Palop, J.J., Yan, F., Cheng, I.H., Wu, T., Gerstein, H., Yu, G.Q., and Mucke, L. (2007). Reducing Endogenous Tau Ameliorates Amyloid B-Induced Deficits in an Alzheimer's Disease Mouse Model. Science 316, 750-754.

Roberts, T.K., Eugenin, E.A., Lopez, L., Romero, I.A., Weksler, B.B., Couraud, P.O., and Berman, J.W. (2012). CCL2 disrupts the adherens junction: implications for neuroinflammation. Laboratory investigation; a journal of technical methods and pathology 92, 1213-1233.

Rogaev, E.I., Sherrington, R., Rogaeva, E.A., Levesque, G., Ikeda, M., Liang, Y., Chi, H., Lin, C., Holman, K., Tsuda, T., et al. (1995). Familial Alzheimer's disease in kindreds with missense mutations in a gene on chromosome 1 related to the Alzheimer's disease type 3 gene. Nature 376, 775-778.

Roy, S.G., Stevens, M.W., So, L., and Edinger, A.L. (2013). Reciprocal effects of rab7 deletion in activated and neglected T cells. Autophagy 9, 1009-1023.

Rupp, N.J., Wegenast-Braun, B.M., Radde, R., Calhoun, M.E., and Jucker, M. (2011). Early onset amyloid lesions lead to severe neuritic abnormalities and local, but not global neuron loss in APPPS1 transgenic mice. Neurobiology of aging 32, 2324.e2321-2326.

Rustenhoven, J., Smith, A.M., Smyth, L.C., Jansson, D., Scotter, E.L., Swanson, M.E.V., Aalderink, M., Coppieters, N., Narayan, P., Handley, R., et al. (2018). PU.1 regulates Alzheimer's diseaseassociated genes in primary human microglia. Molecular neurodegeneration 13, 44.

Saederup, N., Cardona, A.E., Croft, K., Mizutani, M., Cotleur, A.C., Tsou, C.-L., Ransohoff, R.M., and Charo, I.F. (2010). Selective Chemokine Receptor Usage by Central Nervous System Myeloid Cells in CCR2-Red Fluorescent Protein Knock-In Mice. PloS one 5, e13693. 
Safaiyan, S., Kannaiyan, N., Snaidero, N., Brioschi, S., Biber, K., Yona, S., Edinger, A.L., Jung, S., Rossner, M.J., and Simons, M. (2016). Age-related myelin degradation burdens the clearance function of microglia during aging. Nature neuroscience 19, 995-998.

Saido, T.C., Iwatsubo, T., Mann, D.M., Shimada, H., Ihara, Y., and Kawashima, S. (1995). Dominant and differential deposition of distinct beta-amyloid peptide species, A beta N3(pE), in senile plaques. Neuron 14, 457-466.

Sarma, J.D., Ciric, B., Marek, R., Sadhukhan, S., Caruso, M.L., Shafagh, J., Fitzgerald, D.C., Shindler, K.S., and Rostami, A.M. (2009). Functional interleukin-17 receptor A is expressed in central nervous system glia and upregulated in experimental autoimmune encephalomyelitis. Journal of neuroinflammation 6, 14-14.

Scacchi, R., Gambina, G., Broggio, E., Moretto, G., Ruggeri, M., and Corbo, R.M. (2004). The H+ allele of the lipoprotein lipase (LPL) HindllI intronic polymorphism and the risk for sporadic lateonset Alzheimer's disease. Neuroscience letters 367, 177-180.

Schafer, D.P., Lehrman, E.K., Kautzman, A.G., Koyama, R., Mardinly, A.R., Yamasaki, R., Ransohoff, R.M., Greenberg, M.E., Barres, B.A., and Stevens, B. (2012). Microglia sculpt postnatal neural circuits in an activity and complement-dependent manner. Neuron 74, 691-705.

Schilling, S., Lauber, T., Schaupp, M., Manhart, S., Scheel, E., Böhm, G., and Demuth, H.-U. (2006). On the Seeding and Oligomerization of pGlu-Amyloid Peptides (in vitro). Biochemistry 45, 12393-12399.

Schulz, C., Gomez Perdiguero, E., Chorro, L., Szabo-Rogers, H., Cagnard, N., Kierdorf, K., Prinz, M., Wu, B., Jacobsen, S.E., Pollard, J.W., et al. (2012). A lineage of myeloid cells independent of Myb and hematopoietic stem cells. Science 336, 86-90.

Schwarz, J.M., Sholar, P.W., and Bilbo, S.D. (2012). Sex differences in microglial colonization of the developing rat brain. Journal of neurochemistry 120, 948-963.

Selkoe, D.J. (2001). Alzheimer's disease: genes, proteins, and therapy. Physiological reviews 81, 741-766.

Shi, Q., Chowdhury, S., Ma, R., Le, K.X., Hong, S., Caldarone, B.J., Stevens, B., and Lemere, C.A. (2017). Complement C3 deficiency protects against neurodegeneration in aged plaque-rich APP/PS1 mice. Science translational medicine 9.

Shibata, M., Yamada, S., Kumar, S.R., Calero, M., Bading, J., Frangione, B., Holtzman, D.M., Miller, C.A., Strickland, D.K., Ghiso, J., et al. (2000). Clearance of Alzheimer's amyloid-ss(1-40) peptide from brain by LDL receptor-related protein-1 at the blood-brain barrier. The Journal of clinical investigation 106, 1489-1499.

Simard, A.R., Soulet, D., Gowing, G., Julien, J.P., and Rivest, S. (2006). Bone marrow-derived microglia play a critical role in restricting senile plaque formation in Alzheimer's disease. Neuron 49, 489-502.

Söllvander, S., Nikitidou, E., Brolin, R., Söderberg, L., Sehlin, D., Lannfelt, L., and Erlandsson, A. (2016). Accumulation of amyloid- $\beta$ by astrocytes result in enlarged endosomes and microvesicleinduced apoptosis of neurons. Molecular neurodegeneration 11, 38.

Song, E.S., and Hersh, L.B. (2005). Insulysin. Journal of Molecular Neuroscience 25, 201-206. 
Spangenberg, E.E., Lee, R.J., Najafi, A.R., Rice, R.A., Elmore, M.R., Blurton-Jones, M., West, B.L., and Green, K.N. (2016). Eliminating microglia in Alzheimer's mice prevents neuronal loss without modulating amyloid-beta pathology. Brain : a journal of neurology 139, 1265-1281.

Spilman, P., Podlutskaya, N., Hart, M.J., Debnath, J., Gorostiza, O., Bredesen, D., Richardson, A., Strong, R., and Galvan, V. (2010). Inhibition of mTOR by rapamycin abolishes cognitive deficits and reduces amyloid-beta levels in a mouse model of Alzheimer's disease. PloS one 5, e9979.

Spires, T.L., Meyer-Luehmann, M., Stern, E.A., McLean, P.J., Skoch, J., Nguyen, P.T., Bacskai, B.J., and Hyman, B.T. (2005). Dendritic spine abnormalities in APP transgenic mice demonstrated by gene transfer and intravital multiphoton microscopy. The Journal of neuroscience : the official journal of the Society for Neuroscience 25, 7278-7287.

Spittau, B. (2017). Aging Microglia-Phenotypes, Functions and Implications for Age-Related Neurodegenerative Diseases. Frontiers in Aging Neuroscience 9, 194.

Squarzoni, P., Oller, G., Hoeffel, G., Pont-Lezica, L., Rostaing, P., Low, D., Bessis, A., Ginhoux, F., and Garel, S. (2014). Microglia modulate wiring of the embryonic forebrain. Cell Rep 8, 1271-1279.

Stalder, A.K., Ermini, F., Bondolfi, L., Krenger, W., Burbach, G.J., Deller, T., Coomaraswamy, J., Staufenbiel, M., Landmann, R., and Jucker, M. (2005). Invasion of hematopoietic cells into the brain of amyloid precursor protein transgenic mice. J Neurosci 25, 11125-11132.

Stevens, B., Allen, N.J., Vazquez, L.E., Howell, G.R., Christopherson, K.S., Nouri, N., Micheva, K.D., Mehalow, A.K., Huberman, A.D., Stafford, B., et al. (2007). The classical complement cascade mediates CNS synapse elimination. Cell 131, 1164-1178.

Storck, S.E., Meister, S., Nahrath, J., Meissner, J.N., Schubert, N., Di Spiezio, A., Baches, S., Vandenbroucke, R.E., Bouter, Y., Prikulis, I., et al. (2016). Endothelial LRP1 transports amyloidbeta(1-42) across the blood-brain barrier. The Journal of clinical investigation 126, 123-136.

Streit, W.J., Sammons, N.W., Kuhns, A.J., and Sparks, D.L. (2004). Dystrophic microglia in the aging human brain. Glia 45, 208-212.

Strittmatter, W.J., Saunders, A.M., Schmechel, D., Pericak-Vance, M., Enghild, J., Salvesen, G.S., and Roses, A.D. (1993a). Apolipoprotein E: high-avidity binding to beta-amyloid and increased frequency of type 4 allele in late-onset familial Alzheimer disease. Proceedings of the National Academy of Sciences of the United States of America 90.

Strittmatter, W.J., Weisgraber, K.H., Huang, D.Y., Dong, L.M., Salvesen, G.S., Pericak-Vance, M., Schmechel, D., Saunders, A.M., Goldgaber, D., and Roses, A.D. (1993b). Binding of human apolipoprotein $E$ to synthetic amyloid beta peptide: isoform-specific effects and implications for lateonset Alzheimer disease. Proceedings of the National Academy of Sciences of the United States of America 90, 8098-8102.

Styren, S.D., Hamilton, R.L., Styren, G.C., and Klunk, W.E. (2000). X-34, a fluorescent derivative of Congo red: a novel histochemical stain for Alzheimer's disease pathology. The journal of histochemistry and cytochemistry : official journal of the Histochemistry Society 48, 1223-1232.

Sun, B., Zhou, Y., Halabisky, B., Lo, I., Cho, S.H., Mueller-Steiner, S., Devidze, N., Wang, X., Grubb, A., and Gan, L. (2008). Cystatin C-cathepsin B axis regulates amyloid beta levels and associated neuronal deficits in an animal model of Alzheimer's disease. Neuron 60, 247-257.

Sun, S., Zhang, H., Liu, J., Popugaeva, E., Xu, N.-J., Feske, S., White, Charles L., and Bezprozvanny, I. (2014). Reduced Synaptic STIM2 Expression and Impaired Store-Operated Calcium Entry Cause Destabilization of Mature Spines in Mutant Presenilin Mice. Neuron 82, 79-93. 
Sun, X., He, G., and Song, W. (2006). BACE2, as a novel APP theta-secretase, is not responsible for the pathogenesis of Alzheimer's disease in Down syndrome. FASEB journal : official publication of the Federation of American Societies for Experimental Biology 20, 1369-1376.

Sydow, A., Van der Jeugd, A., Zheng, F., Ahmed, T., Balschun, D., Petrova, O., Drexler, D., Zhou, L., Rune, G., Mandelkow, E., et al. (2011). Tau-induced defects in synaptic plasticity, learning, and memory are reversible in transgenic mice after switching off the toxic Tau mutant. J Neurosci 31, 2511-2525.

Tackenberg, C., and Brandt, R. (2009). Divergent Pathways Mediate Spine Alterations and Cell Death Induced by Amyloid- $\beta$, Wild-Type Tau, and R406W Tau. The Journal of Neuroscience 29, 14439-14450.

Takahashi, K., Prinz, M., Stagi, M., Chechneva, O., and Neumann, H. (2007). TREM2-transduced myeloid precursors mediate nervous tissue debris clearance and facilitate recovery in an animal model of multiple sclerosis. PLoS medicine 4, e124.

Takami, M., Nagashima, Y., Sano, Y., Ishihara, S., Morishima-Kawashima, M., Funamoto, S., and Ihara, Y. (2009). gamma-Secretase: successive tripeptide and tetrapeptide release from the transmembrane domain of beta-carboxyl terminal fragment. J Neurosci 29, 13042-13052.

Tay, T.L., Mai, D., Dautzenberg, J., Fernandez-Klett, F., Lin, G., Sagar, Datta, M., Drougard, A., Stempfl, T., Ardura-Fabregat, A., et al. (2017). A new fate mapping system reveals contextdependent random or clonal expansion of microglia. Nature neuroscience 20, 793-803.

Tekirian, T.L., Yang, A.Y., Glabe, C., and Geddes, J.W. (1999). Toxicity of pyroglutaminated amyloid beta-peptides $3(\mathrm{pE})-40$ and -42 is similar to that of $A$ beta1-40 and -42 . Journal of neurochemistry $73,1584-1589$.

Thal, D.R., Rub, U., Orantes, M., and Braak, H. (2002). Phases of A beta-deposition in the human brain and its relevance for the development of AD. Neurology 58, 1791-1800.

Thanopoulou, K., Fragkouli, A., Stylianopoulou, F., and Georgopoulos, S. (2010). Scavenger receptor class B type I (SR-BI) regulates perivascular macrophages and modifies amyloid pathology in an Alzheimer mouse model. Proceedings of the National Academy of Sciences 107, 20816-20821.

Tischer, J., Krueger, M., Mueller, W., Staszewski, O., Prinz, M., Streit, W.J., and Bechmann, I. (2016). Inhomogeneous distribution of Iba-1 characterizes microglial pathology in Alzheimer's disease. Glia 64, 1562-1572.

Trudler, D., Farfara, D., and Frenkel, D. (2010). Toll-like receptors expression and signaling in glia cells in neuro-amyloidogenic diseases: towards future therapeutic application. Mediators of inflammation 2010.

Tsai, J., Grutzendler, J., Duff, K., and Gan, W.B. (2004). Fibrillar amyloid deposition leads to local synaptic abnormalities and breakage of neuronal branches. Nature neuroscience 7, 1181-1183.

Tseng, B.P., Green, K.N., Chan, J.L., Blurton-Jones, M., and LaFerla, F.M. (2008). Abeta inhibits the proteasome and enhances amyloid and tau accumulation. Neurobiology of aging 29, 16071618.

Tzartos, J.S., Friese, M.A., Craner, M.J., Palace, J., Newcombe, J., Esiri, M.M., and Fugger, L. (2008). Interleukin-17 Production in Central Nervous System-Infiltrating T Cells and Glial Cells Is Associated with Active Disease in Multiple Sclerosis. The American journal of pathology 172, 146155. 
Uchihara, T., Duyckaerts, C., He, Y., Kobayashi, K., Seilhean, D., Amouyel, P., and Hauw, J.J. (1995). ApoE immunoreactivity and microglial cells in Alzheimer's disease brain. Neuroscience letters 195, 5-8.

Ulland, T.K., Song, W.M., Huang, S.C.-C., Ulrich, J.D., Sergushichev, A., Beatty, W.L., Loboda, A.A., Zhou, Y., Cairns, N.J., Kambal, A., et al. (2017). TREM2 Maintains Microglial Metabolic Fitness in Alzheimer\&\#x2019;s Disease. Cell 170, 649-663.e613.

Ulrich, J.D., Ulland, T.K., Mahan, T.E., Nyström, S., Nilsson, K.P., Song, W.M., Zhou, Y., Reinartz, M., Choi, S., Jiang, H., et al. (2018). ApoE facilitates the microglial response to amyloid plaque pathology. The Journal of experimental medicine.

Umeda, T., Tomiyama, T., Sakama, N., Tanaka, S., Lambert, M.P., Klein, W.L., and Mori, H. (2011). Intraneuronal amyloid beta oligomers cause cell death via endoplasmic reticulum stress, endosomal/lysosomal leakage, and mitochondrial dysfunction in vivo. J Neurosci Res 89, 10311042.

VanGuilder, H.D., Bixler, G.V., Brucklacher, R.M., Farley, J.A., Yan, H., Warrington, J.P., Sonntag, W.E., and Freeman, W.M. (2011). Concurrent hippocampal induction of MHC II pathway components and glial activation with advanced aging is not correlated with cognitive impairment. Journal of neuroinflammation 8,138 .

Varvel, N.H., Neher, J.J., Bosch, A., Wang, W., Ransohoff, R.M., Miller, R.J., and Dingledine, R. (2016). Infiltrating monocytes promote brain inflammation and exacerbate neuronal damage after status epilepticus. Proceedings of the National Academy of Sciences of the United States of America 113, E5665-E5674.

Vay, S.U., Flitsch, L.J., Rabenstein, M., Rogall, R., Blaschke, S., Kleinhaus, J., Reinert, N., Bach, A., Fink, G.R., Schroeter, M., et al. (2018). The plasticity of primary microglia and their multifaceted effects on endogenous neural stem cells in vitro and in vivo. Journal of neuroinflammation 15, 226.

Venegas, C., Kumar, S., Franklin, B.S., Dierkes, T., Brinkschulte, R., Tejera, D., Vieira-Saecker, A., Schwartz, S., Santarelli, F., Kummer, M.P., et al. (2017). Microglia-derived ASC specks cross-seed amyloid- $\beta$ in Alzheimer's disease. Nature 552, 355.

Verhoeven, K., De Jonghe, P., Coen, K., Verpoorten, N., Auer-Grumbach, M., Kwon, J.M., FitzPatrick, D., Schmedding, E., De Vriendt, E., Jacobs, A., et al. (2003). Mutations in the small GTP-ase late endosomal protein RAB7 cause Charcot-Marie-Tooth type 2B neuropathy. Am J Hum Genet 72, 722-727.

Villa, A., Gelosa, P., Castiglioni, L., Cimino, M., Rizzi, N., Pepe, G., Lolli, F., Marcello, E., Sironi, L., Vegeto, E., et al. (2018). Sex-Specific Features of Microglia from Adult Mice. Cell Reports 23, 35013511.

Vogel, D.Y.S., Kooij, G., Heijnen, P.D.A.M., Breur, M., Peferoen, L.A.N., Valk, P., Vries, H.E., Amor, S., and Dijkstra, C.D. (2015). GM-CSF promotes migration of human monocytes across the blood brain barrier. European Journal of Immunology 45, 1808-1819.

von Bernhardi, R., Eugenín-von Bernhardi, L., and Eugenín, J. (2015). Microglial cell dysregulation in brain aging and neurodegeneration. Frontiers in Aging Neuroscience 7, 124.

Walsh, D.M., Klyubin, I., Fadeeva, J.V., Cullen, W.K., Anwyl, R., Wolfe, M.S., Rowan, M.J., and Selkoe, D.J. (2002). Naturally secreted oligomers of amyloid beta protein potently inhibit hippocampal long-term potentiation in vivo. Nature 416, 535-539. 
Wandinger-Ness, A., and Zerial, M. (2014). Rab Proteins and the Compartmentalization of the Endosomal System. Cold Spring Harbor Perspectives in Biology 6, a022616.

Wang, H., and Eckel, R.H. (2012). Lipoprotein Lipase in the Brain and Nervous System. Annual review of nutrition 32, 147-160.

Wang, H., Sang, N., Zhang, C., Raghupathi, R., Tanzi, R.E., and Saunders, A. (2015a). Cathepsin L Mediates the Degradation of Novel APP C-Terminal Fragments. Biochemistry 54, 2806-2816.

Wang, J., Tanila, H., Puolivali, J., Kadish, I., and van Groen, T. (2003). Gender differences in the amount and deposition of amyloidbeta in APPswe and PS1 double transgenic mice. Neurobiology of disease $14,318-327$.

Wang, Y., Cella, M., Mallinson, K., Ulrich, J.D., Young, K.L., Robinette, M.L., Gilfillan, S., Krishnan, G.M., Sudhakar, S., Zinselmeyer, B.H., et al. (2015b). TREM2 lipid sensing sustains the microglial response in an Alzheimer's disease model. Cell 160, 1061-1071.

Wang, Y., and Mandelkow, E. (2016). Tau in physiology and pathology. Nature reviews Neuroscience 17, 5-21.

Wang, Y., Ulland, T.K., Ulrich, J.D., Song, W., Tzaferis, J.A., Hole, J.T., Yuan, P., Mahan, T.E., Shi, Y., Gilfillan, S., et al. (2016). TREM2-mediated early microglial response limits diffusion and toxicity of amyloid plaques. The Journal of experimental medicine 213, 667-675.

Warner, C.J., Dutta, S., Foley, A.R., and Raskatov, J.A. (2016). Introduction of d-Glutamate at a Critical Residue of Abeta42 Stabilizes a Prefibrillary Aggregate with Enhanced Toxicity. Chemistry (Weinheim an der Bergstrasse, Germany) 22, 11967-11970.

Weggen, S., and Beher, D. (2012). Molecular consequences of amyloid precursor protein and presenilin mutations causing autosomal-dominant Alzheimer's disease. Alzheimer's research \& therapy $4,9-9$.

Weingarten, M.D., Lockwood, A.H., Hwo, S.Y., and Kirschner, M.W. (1975). A protein factor essential for microtubule assembly. Proceedings of the National Academy of Sciences of the United States of America 72, 1858-1862.

Weuve, J., Hebert, L.E., Scherr, P.A., and Evans, D.A. (2014). Deaths in the United States among persons with Alzheimer's disease (2010-2050). Alzheimer's \& dementia : the journal of the Alzheimer's Association 10, e40-46.

Whitehouse, P.J., Price, D.L., Struble, R.G., Clark, A.W., Coyle, J.T., and Delon, M.R. (1982). Alzheimer's disease and senile dementia: loss of neurons in the basal forebrain. Science 215, 1237-1239.

Willem, M., Tahirovic, S., Busche, M.A., Ovsepian, S.V., Chafai, M., Kootar, S., Hornburg, D., Evans, L.D., Moore, S., Daria, A., et al. (2015). eta-Secretase processing of APP inhibits neuronal activity in the hippocampus. Nature 526, 443-447.

Wippold, F.J., Cairns, N., Vo, K., Holtzman, D.M., and Morris, J.C. (2008). Neuropathology for the Neuroradiologist: Plaques and Tangles. American Journal of Neuroradiology 29, 18-22.

Wisniewski, H.M., Barcikowska, M., and Kida, E. (1991). Phagocytosis of beta/A4 amyloid fibrils of the neuritic neocortical plaques. Acta neuropathologica 81, 588-590. 
Wisniewski, H.M., Ghetti, B., and Terry, R.D. (1973). Neuritic (senile) plaques and filamentous changes in aged rhesus monkeys. Journal of neuropathology and experimental neurology $32,566-$ 584.

Wolf, Y., Yona, S., Kim, K.-W., and Jung, S. (2013). Microglia, seen from the CX(3)CR1 angle. Frontiers in cellular neuroscience 7,26.

Wolfe, M.S. (2009). Tau mutations in neurodegenerative diseases. The Journal of biological chemistry 284, 6021-6025.

Wong, A.M., Patel, N.V., Patel, N.K., Wei, M., Morgan, T.E., de Beer, M.C., de Villiers, W.J., and Finch, C.E. (2005). Macrosialin increases during normal brain aging are attenuated by caloric restriction. Neuroscience letters 390, 76-80.

Wong, C.-O., Gregory, S., Hu, H., Chao, Y., Sepúlveda, V.E., He, Y., Li-Kroeger, D., Goldman, W.E., Bellen, H.J., and Venkatachalam, K. (2017). Lysosomal Degradation Is Required for Sustained Phagocytosis of Bacteria by Macrophages. Cell Host \& Microbe 21, 719-730.e716.

Wong, K.T., Robertson, T., Ong, B.B., Chong, J.W., Yaiw, K.C., Wang, L.F., Ansford, A.J., and Tannenberg, A. (2009). Human Hendra virus infection causes acute and relapsing encephalitis. Neuropathology and applied neurobiology 35, 296-305.

Wood, L.B., Winslow, A.R., Proctor, E.A., McGuone, D., Mordes, D.A., Frosch, M.P., Hyman, B.T., Lauffenburger, D.A., and Haigis, K.M. (2015). Identification of neurotoxic cytokines by profiling Alzheimer's disease tissues and neuron culture viability screening. Scientific reports 5, 16622.

Wyss-Coray, T., Loike, J.D., Brionne, T.C., Lu, E., Anankov, R., Yan, F., Silverstein, S.C., and Husemann, J. (2003). Adult mouse astrocytes degrade amyloid-beta in vitro and in situ. Nature medicine 9, 453-457.

Wyss-Coray, T., Yan, F., Lin, A.H., Lambris, J.D., Alexander, J.J., Quigg, R.J., and Masliah, E. (2002). Prominent neurodegeneration and increased plaque formation in complement-inhibited Alzheimer's mice. Proceedings of the National Academy of Sciences of the United States of America 99, 10837-10842.

Xiao, Q., Yan, P., Ma, X., Liu, H., Perez, R., Zhu, A., Gonzales, E., Burchett, J.M., Schuler, D.R., Cirrito, J.R., et al. (2014). Enhancing astrocytic lysosome biogenesis facilitates Abeta clearance and attenuates amyloid plaque pathogenesis. J Neurosci 34, 9607-9620.

Yan, P., Hu, X., Song, H., Yin, K., Bateman, R.J., Cirrito, J.R., Xiao, Q., Hsu, F.F., Turk, J.W., Xu, J., et al. (2006). Matrix metalloproteinase-9 degrades amyloid-beta fibrils in vitro and compact plaques in situ. The Journal of biological chemistry $281,24566-24574$.

Yang, A.J., Chandswangbhuvana, D., Margol, L., and Glabe, C.G. (1998). Loss of endosomal/lysosomal membrane impermeability is an early event in amyloid Abeta1-42 pathogenesis. J Neurosci Res 52, 691-698.

Yang, D.S., Stavrides, P., Mohan, P.S., Kaushik, S., Kumar, A., Ohno, M., Schmidt, S.D., Wesson, D., Bandyopadhyay, U., Jiang, Y., et al. (2011). Reversal of autophagy dysfunction in the TgCRND8 mouse model of Alzheimer's disease ameliorates amyloid pathologies and memory deficits. Brain : a journal of neurology $134,258-277$.

Yang, T., Li, S., Xu, H., Walsh, D.M., and Selkoe, D.J. (2017). Large Soluble Oligomers of Amyloid beta-Protein from Alzheimer Brain Are Far Less Neuroactive Than the Smaller Oligomers to Which They Dissociate. J Neurosci 37, 152-163. 
Yanguas-Casas, N., Crespo-Castrillo, A., de Ceballos, M.L., Chowen, J.A., Azcoitia, I., Arevalo, M.A., and Garcia-Segura, L.M. (2018). Sex differences in the phagocytic and migratory activity of microglia and their impairment by palmitic acid. Glia 66, 522-537.

Yao, L., Kan, E.M., Lu, J., Hao, A., Dheen, S.T., Kaur, C., and Ling, E.A. (2013). Toll-like receptor 4 mediates microglial activation and production of inflammatory mediators in neonatal rat brain following hypoxia: role of TLR4 in hypoxic microglia. Journal of neuroinflammation 10, 23.

Yeh, F.L., Wang, Y., Tom, I., Gonzalez, L.C., and Sheng, M. (2016). TREM2 Binds to Apolipoproteins, Including APOE and CLU/APOJ, and Thereby Facilitates Uptake of Amyloid-Beta by Microglia. Neuron 91, 328-340.

Yepes, M., Sandkvist, M., Moore, E.G., Bugge, T.H., Strickland, D.K., and Lawrence, D.A. (2003). Tissue-type plasminogen activator induces opening of the blood-brain barrier via the LDL receptorrelated protein. The Journal of clinical investigation 112, 1533-1540.

Yin, F., Banerjee, R., Thomas, B., Zhou, P., Qian, L., Jia, T., Ma, X., Ma, Y., ladecola, C., Beal, M.F., et al. (2010). Exaggerated inflammation, impaired host defense, and neuropathology in progranulin-deficient mice. The Journal of experimental medicine 207, 117-128.

Yin, Z., Raj, D., Saiepour, N., Van Dam, D., Brouwer, N., Holtman, I.R., Eggen, B.J.L., Moller, T., Tamm, J.A., Abdourahman, A., et al. (2017). Immune hyperreactivity of Abeta plaque-associated microglia in Alzheimer's disease. Neurobiology of aging 55, 115-122.

Yona, S., Kim, K.W., Wolf, Y., Mildner, A., Varol, D., Breker, M., Strauss-Ayali, D., Viukov, S., Guilliams, M., Misharin, A., et al. (2013). Fate mapping reveals origins and dynamics of monocytes and tissue macrophages under homeostasis. Immunity 38, 79-91.

Yoshikai, S.-i., Sasaki, H., Doh-ura, K., Furuya, H., and Sakaki, Y. (1990). Genomic organization of the human amyloid beta-protein precursor gene. Gene 87, 257-263.

Youssef, I., Florent-Bechard, S., Malaplate-Armand, C., Koziel, V., Bihain, B., Olivier, J.L., Leininger-Muller, B., Kriem, B., Oster, T., and Pillot, T. (2008). N-truncated amyloid-beta oligomers induce learning impairment and neuronal apoptosis. Neurobiology of aging 29, 1319-1333.

Yu, L., McPhee, C.K., Zheng, L., Mardones, G.A., Rong, Y., Peng, J., Mi, N., Zhao, Y., Liu, Z., Wan, F., et al. (2010). Termination of autophagy and reformation of lysosomes regulated by mTOR. Nature 465, 942.

Zaqout, S., and Kaindl, A.M. (2016). Golgi-Cox Staining Step by Step. Frontiers in Neuroanatomy $10,38$.

Zeisel, A., Muñoz-Manchado, A.B., Codeluppi, S., Lönnerberg, P., La Manno, G., Juréus, A., Marques, S., Munguba, H., He, L., Betsholtz, C., et al. (2015). Cell types in the mouse cortex and hippocampus revealed by single-cell RNA-seq. Science 347, 1138-1142.

Zempel, H., Luedtke, J., Kumar, Y., Biernat, J., Dawson, H., Mandelkow, E., and Mandelkow, E.-M. (2013). Amyloid- $\beta$ oligomers induce synaptic damage via Tau-dependent microtubule severing by TTLL6 and spastin. The EMBO journal 32, 2920-2937.

Zenaro, E., Piacentino, G., and Constantin, G. (2017). The blood-brain barrier in Alzheimer's disease. Neurobiology of disease 107, 41-56.

Zenaro, E., Pietronigro, E., Della Bianca, V., Piacentino, G., Marongiu, L., Budui, S., Turano, E., Rossi, B., Angiari, S., Dusi, S., et al. (2015). Neutrophils promote Alzheimer's disease-like pathology and cognitive decline via LFA-1 integrin. Nature medicine 21, 880-886. 
Zhang, Z., Song, M., Liu, X., Su Kang, S., Duong, D.M., Seyfried, N.T., Cao, X., Cheng, L., Sun, Y.E., Ping Yu, S., et al. (2015). Delta-secretase cleaves amyloid precursor protein and regulates the pathogenesis in Alzheimer's disease. Nat Commun 6, 8762.

Zhao, R., Hu, W., Tsai, J., Li, W., and Gan, W.-B. (2017). Microglia limit the expansion of $\beta$-amyloid plaques in a mouse model of Alzheimer's disease. Molecular neurodegeneration 12, 47.

Zhao, Y., Wu, X., Li, X., Jiang, L.-L., Gui, X., Liu, Y., Sun, Y., Zhu, B., Piña-Crespo, J.C., Zhang, M., et al. (2018). TREM2 Is a Receptor for $\beta$-Amyloid that Mediates Microglial Function. Neuron 97, 1023-1031.e1027.

Zhou, J., Fonseca, M.I., Pisalyaput, K., and Tenner, A.J. (2008). Complement C3 and C4 expression in $\mathrm{C} 1 \mathrm{q}$ sufficient and deficient mouse models of Alzheimer's disease. Journal of neurochemistry 106, 2080-2092. 


\section{Curriculum Vitae}

\section{Beate Maren Erika Koch}

Personal details:

Date of birth: $\quad$ November $20^{\text {th }}, 1988$

Place of birth: $\quad$ Schwäbisch Hall, Germany

Mail address: $\quad$ Beate.koch@dzne.de

\section{Education:}

12/2014 - present

PhD student in GGNB PhD program "Molecular Physiology of the Brain"

Georg-August University Göttingen, Germany

$11 / 2012-11 / 2014$

Master in Molecular Medicine

Friedrich-August University Erlangen-Nürnberg, Germany

09/2013 - 01/2014 M2 Master of Science in Neurobiology

Université Pierre et Marie Curie, Paris, France

10/2009 - 10/2012 Bachelor in Molecular Medicine

Friedrich-August University Erlangen-Nürnberg, Germany

10/2008 - 09/2009 Bachelor studies in Life Science Engineering

Friedrich-August University Erlangen-Nürnberg, Germany

08/1999 - 06/2008 „Allgemeine Hochschulreife“

Schenk-von-Limburg Gymnasium, Gaildorf

Professional Experience:

12/2014 - present

PhD student at the German Center for Neurodegenerative Diseases (DZNE) Göttingen / Bonn, Germany

Thesis: "Characterization of microglial Rab7 knockout on amyloid pathology in the 5xFAD mouse model of Alzheimer's disease"

Advisor: Prof. Dr. Anja Schneider

06/2015 - 02/2016 Visiting Researcher at Stanford University, USA

Research project: "Effects of plasma and plasma exosomes from aged donors on neurogenesis and neuroinflammation"

Advisors: Prof. Dr. Tony Wyss-Coray and Prof. Dr. Anja Schneider 
11/2012 - 11/2014 Master Thesis at Friedrich-August University Erlangen-Nürnberg, Germany

Master thesis: "Sox10 overexpression throughout the spinal cord causes premature oligodendrocyte specification and differentiation"

Advisor: Prof. Dr. Michael Wegner

09/2013 - 12/2013 Research Internship

Pasteur Institute, Paris, France

Research project: "Functional and morphological influences of amyloid beta on cholinergic neurons"

Advisor: Prof. Dr. Uwe Maskos

10/2012 - 08/2013 Student Research Associate

Friedrich-August University Erlangen-Nürnberg, Germany

Research projects in molecular pneumology focusing on asthma and pulmonary cancer

Advisor: Prof. Dr. Dr. Susetta Finotto

10/2009-10/2012 Bachelor Thesis at Friedrich-August University ErlangenNürnberg, Germany

Bachelor thesis: "Molekulargenetische Analysen zu der Expression von Tas1r1 in Testisgewebe unterschiedlicher Mausstämme" (engl. Molecular genetic analysis on the expression of Tas1R1 in testis of different mouse strains)

Advisor: Prof. Dr. Andreas Winterpacht

\section{Publications:}

Martin Schicht, Felix Rausch, Susetta Finotto, Martina Mathews, Anja Mattil, Melanie Schubert, Beate Koch, Maximilian Traxdorf, Christopher Bohr, Dieter Worlitzsch, Wolfgang Brandt, Fabian Garreis, Saadettin Sel, Friedrich Paulsen and Lars Bräuer (2014). SFTA3, a novel protein of the lung: three-dimensional structure, characterisation and immune activation. European Respiratory Journal 44 (2). 